


\title{
SKELETAL TISSUE ENGINEERING USING EMBRYONIC STEM CELLS
}

\author{
PROEFSCHRIFT \\ ter verkrijging van \\ de graad van doctor aan de Universiteit Twente, \\ op gezag van de rector magnificus, \\ prof. dr. H. Brinksma, \\ volgens besluit van het College voor Promoties \\ in het openbaar te verdedigen \\ op donderdag 22 oktober 2009 om 16.45 uur
}

door

Jojanneke Maria Jukes

geboren op 28 maart 1978

te Eindhoven 
Dit proefschrift is goedgekeurd door:

Promotor: $\quad$ Prof. dr. Clemens A. van Blitterswijk

Co-promotor: Dr. Jan de Boer

Copyright: 2009, Jojanneke Maria Jukes, Enschede, The Netherlands.

Neither this thesis nor its parts may be reproduced without written permission of the author.

ISBN: 978-90-365-2887-0 
SKELETAL TISSUE ENGINEERING USING

EMBRYONIC STEM CELLS

\section{SKELETWEEFSELKWEEK MET EMBRYONALE \\ STAMCELLEN}




\section{LEDEN VAN DE PROMOTIECOMMISSIE}

\author{
Voorzitter: \\ Prof. dr. G. van der Steenhoven \\ Promotor: \\ Prof. dr. C.A. van Blitterswijk \\ Co-promotor: \\ Dr. J. de Boer \\ Leden: \\ Prof. dr. L.W.M.M. Terstappen \\ Dr. P.J. Dijkstra \\ Prof. dr. G. de Haan \\ Prof. dr. A. Lindahl \\ Dr. D.B.F. Saris \\ Dr. G.J.V.M. van Osch
}

\author{
University of Twente \\ University of Twente \\ University of Twente \\ University of Twente \\ University of Twente \\ University Medical Center Groningen \\ Göteborg University \\ University Medical Center Utrecht \\ Erasmus Medical Center Rotterdam
}

Jojanneke Maria Jukes

Skeletal tissue engineering using embryonic stem cells

The research described in this thesis was financially supported by the Dutch Technology Foundation STW (TPG 5923).

The publication of this thesis was sponsored by the Netherlands Society for Biomaterials and Tissue Engineering and the Anna fonds.
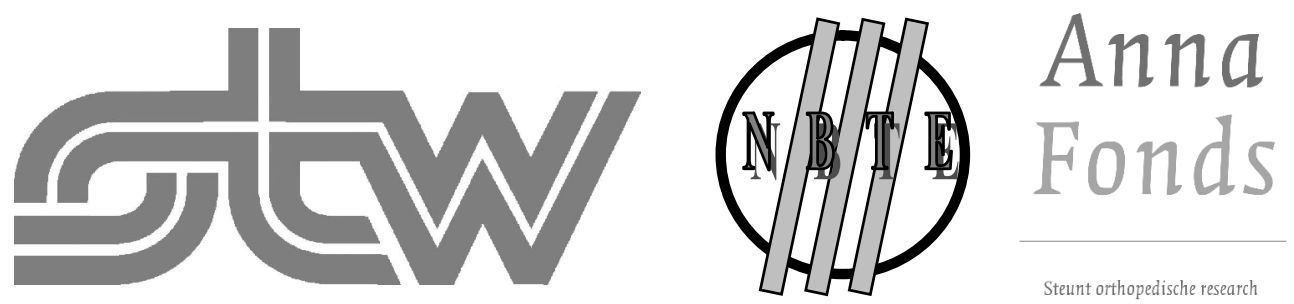

Drukwerk: Wöhrmann Print Service, Zutphen, Nederland.

Omslag: Roze, de kleur die weergaf dat ik succesvol kraakbeen en bot had gevormd uit muizen en menselijke embryonale stamcellen. 


\section{CONTENTS}

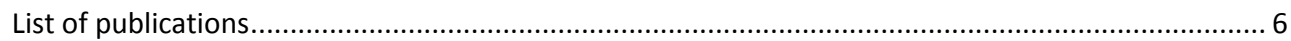

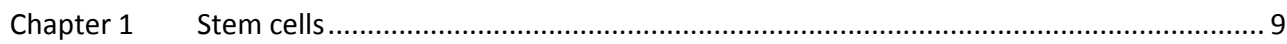

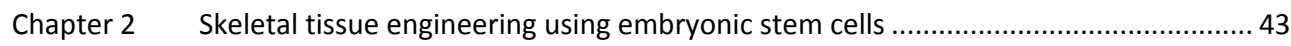

Chapter 3 A newly developed chemically crosslinked Dex-PEG hydrogel for cartilage

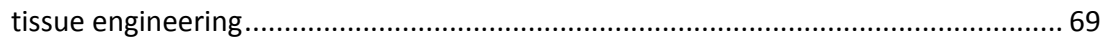

Chapter 4 Critical steps toward a tissue-engineered cartilage implant using embryonic

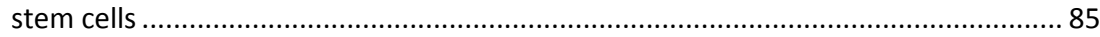

Chapter 5 Efficiency of cartilage formation by mouse and human embryonic stem cells.......... 105

Chapter 6 Endochondral bone tissue engineering using embryonic stem cells ........................ 121

Chapter $7 \quad$ Potential of embryonic stem cells for in vivo bone regeneration ............................... 137

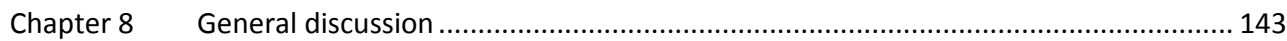

Summary

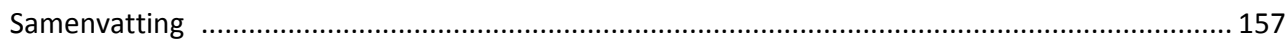

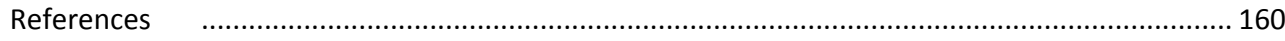

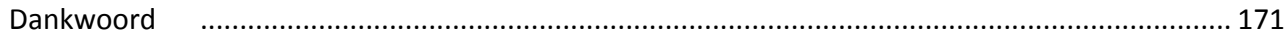

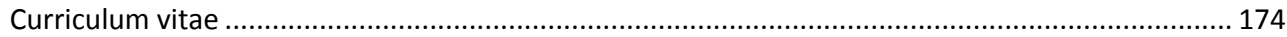




\section{LIST OF PUBLICATIONS}

\section{This thesis is based on the following publications}

\section{Peer reviewed papers}

- Jukes, JM, Both, SK, Post, JN, Blitterswijk, CA, Karperien M and de Boer J. Chapter 1 Stem cells. Tissue engineering 2008; Academic press Elsevier: ISBN 978-0-12-370869-4

- Jukes, JM, van Blitterswijk, CA and de Boer, J. Skeletal tissue engineering using embryonic stem cells. Submitted

- Jukes, JM, van der Aa, LJ, Hiemstra, C, van Veen, T, Dijkstra, PJ, Zhong, Z, Feijen, J, van Blitterswijk, CA and de Boer, J. A newly developed chemically crosslinked Dex-PEG hydrogel for cartilage tissue engineering. Tissue Engineering, accepted

- Jukes, JM, Moroni, L, van Blitterswijk, CA and de Boer, J. Critical steps toward a tissueengineered cartilage implant using embryonic stem cells. Tissue Eng Part A 2008;14:135-47

- Jukes, JM, Both, SK, Leusink, A, Sterk, LM, van Blitterswijk, CA and de Boer, J. Endochondral bone tissue engineering using embryonic stem cells. Proc Natl Acad Sci U S A 2008;105:6840-5

- Jukes, JM, Both, SK, van Blitterswijk, CA and de Boer, J. Potential of embryonic stem cells for in vivo bone regeneration. Regen Med 2008;3:783-5

\section{Selected abstracts}

- Jukes, JM, van Blitterswijk, CA and de Boer J. Chondrogenic differentiation of mouse embryonic stem cells on polymeric scaffolds. Dutch annual conference on biomedical engineering 2004; Papendal, The Netherlands: Oral presentation

- Jukes, JM, van Blitterswijk, CA and de Boer, J. Chondrogenic differentiation of mouse embryonic stem cells on polymeric scaffolds. $13^{\mathrm{e}}$ Conferentie van Nederlandse Vereniging voor Biomaterialen en Tissue Engineering 2004; Lunteren, The Netherlands: Oral presentation

- Jukes, JM, Moroni, L, van Blitterswijk CA and de Boer J. Cartilage formation by mouse embryonic stem cell on biodegradable scaffolds. $4^{\text {th }}$ annual meeting of the European Tissue Engineering Society 2005; Munich, Germany: Oral presentation

- Jukes, JM, Both, SK, van Blitterswijk CA and de Boer J. Bone and cartilage tissue engineering using embryonic stem cells. DPTE Symposium on stem cells 2006; Utrecht, The Netherlands: Oral presentation 
- Jukes, JM, Moroni, L, van Blitterswijk, CA and de Boer, J. Design of a tissue-engineered cartilage implant using embryonic stem cells. $4^{\text {th }}$ Meeting of the International Society for Stem Cell Research (ISSCR) 2006; Toronto, Canada: Poster presentation

- Jukes, JM, Both, SK, van Blitterswijk CA and de Boer J. Endochondral bone formation by embryonic stem cells on scaffolds in vivo. Tissue Engineering and Regenerative Medicine International Society European Chapter (TERMIS-EU) Meeting 2006; Rotterdam, The Netherlands: Poster presentation

- Jukes, JM, Both, SK, van Blitterswijk, CA and de Boer, J. Endochondral bone formation by embryonic stem cells on scaffolds in vivo. $15^{\mathrm{e}}$ Conferentie van Nederlandse Vereniging voor Biomaterialen en Tissue Engineering 2006; Lunteren, The Netherlands: Oral presentation

- Jukes, JM, Both, SK, van Blitterswijk, CA and de Boer, J. ESCs in skeletal tissue engineering. 4th Marie Curie Cutting Edge InVENTS Conference on Biocompatibility evaluation and biological behavior of polymeric biomaterials 2007; Alvor, Portugal: Oral presentation 



\section{CHAPTER 1 STEM CELLS}

Jojanneke M. Jukes ${ }^{1}$, Sanne K. Both ${ }^{1}$, Janine N. Post ${ }^{2}$, Clemens A. van Blitterswijk ${ }^{1}$ Marcel Karperien ${ }^{1}$, Jan de Boer ${ }^{1}$

${ }^{1}$ Institute for Biomedical Technology, Department of Tissue Regeneration, University of Twente, Enschede, The Netherlands

${ }^{2}$ Institute for Biomedical Technology, Department of Polymer Chemistry and Biomaterials, University of Twente, Enschede, The Netherlands

Nobody said it was easy

The Scientist - Coldplay - A rush of blood to the head 


\section{CHAPTER 1 STEM CELLS}

"The essence of knowledge is, having it, to apply it; not having it, to confess your ignorance" Confucius

\section{Snapshot summary}

- Two defining properties of stem cells are their ability to self-renew and their ability to differentiate.

- Self-renewal is orchestrated by a complex network of intrinsic and extrinsic factors, which are species- and tissue-specific.

- Embryonic stem cells are pluripotent. Most adult stem cells are multipotent.

- Differentiation of cells is not always a one-way street. Cell fates can be reset by epigenetic reprogramming.

- $\quad$ Adult stem cells might display plasticity.

- Embryonic stem cells are isolated from the inner cell mass of a blastocyst and exist only in vitro. Adult stem cells can be isolated from various tissues.

- Embryonic stem cells have to be characterized in vitro and in vivo, to confirm their self-renewal capacity and pluripotency.

- Adult stem cells are rare and stem cell division rate is low in the body.

- The stem cell niche is the micro-environment where the stem cells reside in vivo.

- Mesenchymal stem cells go into replicative senescence when cultured in vitro. 


\section{What defines a stem cell?}

Stem cells can be defined by two properties: the ability to make identical copies of themselves (selfrenewal) and the ability to form other cell types of the body (differentiation) (Figure 1). These properties are also referred to as stemness. Stem cells may potentially provide an unlimited supply of cells that can form any of the hundreds of specialized cells in the body. It is because of these properties that stem cells are an interesting cell source for tissue engineers.

Stem cells can be divided into two main groups: embryonic and adult or somatic stem cells. Embryonic stem cells (ESCs) are responsible for embryonic and fetal development and growth. In the human body, adult stem cells are responsible for growth, tissue maintenance and regeneration and repair of diseased or damaged tissue.
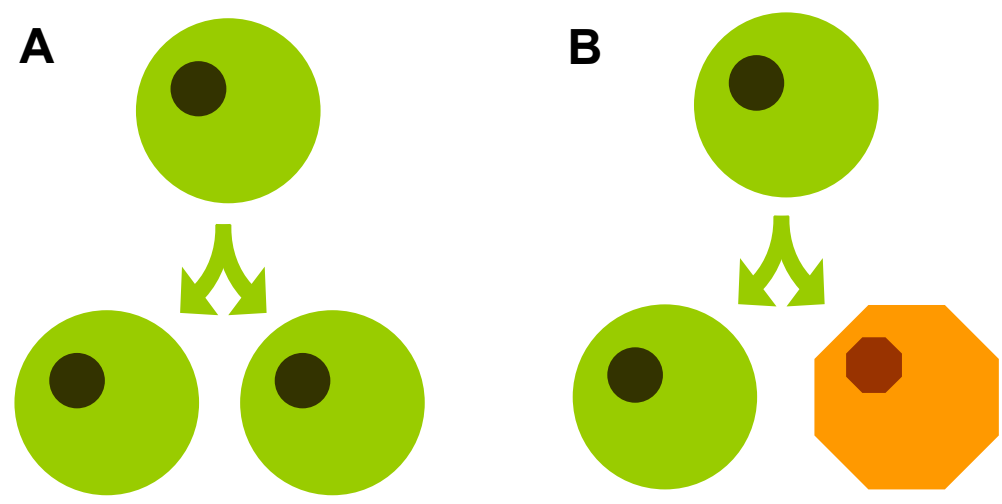

Figure 1. Stem cell characteristics

Upon cell division, a stem cell (green circle) can produce a new stem cell (self-renewal), and a differentiated daughter cell (orange octagon). A) Symmetrical cell division and B) asymmetrical cell division.

\subsection{Stem cell self-renewal}

During a stem cell division, one or both daughter cells maintain the stem cell phenotype. The process is called self-renewal. Stem cells can divide symmetrically or asymmetrically. It is the balance between symmetrical and asymmetrical divisions that determines the appropriate numbers of stem cells and differentiated daughters.

During a symmetric cell division, both daughter cells acquire the same fate; either undifferentiated (new stem cells) or differentiated.

During an asymmetric cell division, one daughter cell becomes a new stem cell; the other differentiates into a more specialized cell type (Figure 1 and 2). Asymmetric cell divisions are 
controlled by intrinsic and extrinsic mechanisms. Intrinsic mechanisms rely on the asymmetric partitioning of cell components, such as cell polarity factors or cell fate determinants. In the extrinsic mechanism, the two daughter cells are positioned asymmetrically in their environment and receive different external signals [1].

a

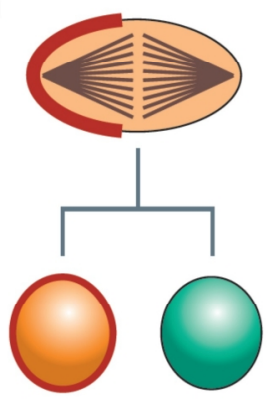

b

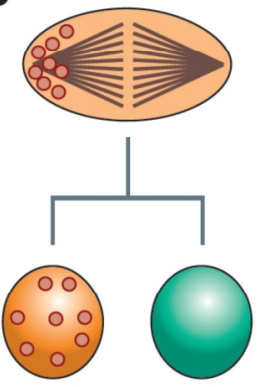

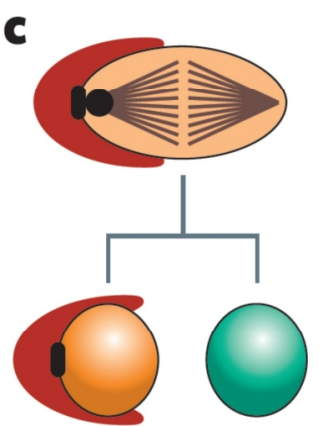

Figure 2. Three simple mechanisms of asymmetrical cell division

Stem cells are orange, differentiated cells are green. A) Asymmetric localization of cell polarity regulators (red) initiates the asymmetric division. B) Cell fate determinants (red) can be segregated to the cytoplasm of one daughter cell, as shown here, or they can be associated with the membrane, centrosome or another cellular constituent that is differentially distributed to the daughters. C) Regulated orientation of the mitotic spindle retains only one daughter in the stem-cell niche (red), such that only that daughter cell has access to extrinsic signals necessary for maintaining stem-cell identity. This mechanism achieves an asymmetric outcome, even though the division itself is intrinsically symmetric. In an alternative but similar model, the daughter cell placed away from the niche is exposed to signals that induce differentiation [1].

The past 25 years of research have given some insight into the mechanism by which a cell maintains its undifferentiated fate. Since self-renewal involves both proliferation and the maintenance of an undifferentiated phenotype, multiple pathways are involved. Stem cells from different tissues or at different stages of developmental potential (pluripotent or multipotent) use different mechanisms to regulate self-renewal. The pathways regulating self-renewal are depending on the context. Factors that might stimulate differentiation of one cell type, might be involved in the maintenance of self-renewal of another stem cell. Some mechanisms and interactions are still unknown, some are debatable, and others are well-described. Self-renewal of embryonic and adult stem cells is described in section 2.3 and 3.3.

\subsection{Differentiation}

\subsubsection{Can a stem cell become everything it wants to be?}

The second defining property of a stem cell is its ability to differentiate into a more specialized cell.

The number of cells types a stem cell can differentiate into is determined by its potency: 
- Totipotent stem cells have the ability to form an entire organism. The fertilized oocyte and the cells after the first cleavage divisions are considered totipotent.

- Pluripotent stem cells are able to form all 3 germ layers including germ cells, but not the extraembryonic tissue as placenta and umbilical cord. Cells of the inner cell mass of the blastocyst are pluripotent. When these cells are brought into culture, they are called embryonic stem cells.

- Multipotency means the ability to form multiple cell types. Mesenchymal stem cells can differentiate into cells that form bone, cartilage and fat.

- Oligopotent stem cells can differentiate into two or more lineages, for example neural stem cells that can form a subset of neurons in the brain.

- Unipotency is the ability to form cells from a single lineage, for example spermatogonial stem cells.

The term omnipotence is not used for stem cells, but is used in religions as one of God's characteristics.

\subsubsection{Stem cells, precursor cells and differentiated cells}

Once a stem cell leaves its niche (section 3.4) and is no longer under control of intrinsic and extrinsic factors that maintain the undifferentiated phenotype, they will start to differentiate. This cell will become a progenitor or precursor cell, or a transit amplifying cell ("transit", because they are in transit from a stem cell to a differentiated cell; "amplifying" because the continuing cell divisions amplify the number of differentiated progeny). The committed cell can differentiate further along a specific lineage, until it is terminally differentiated into the mature phenotype (Figure 3 ). These cells are presumably irreversibly blocked in their ability to proliferate, but they can perform specialized functions for a long period of time before they die.

The progenitor cells can divide many times, ultimately giving rise to thousands of fully differentiated cells that have originated from one stem cell division. This explains why the number of stem cells is so small and that stem cell division rate is low. For example, in bone marrow only an estimated 1 in 10,000 to 15,000 cells is considered to be a stem cell. Nevertheless, billions of new blood cells are formed every day. 


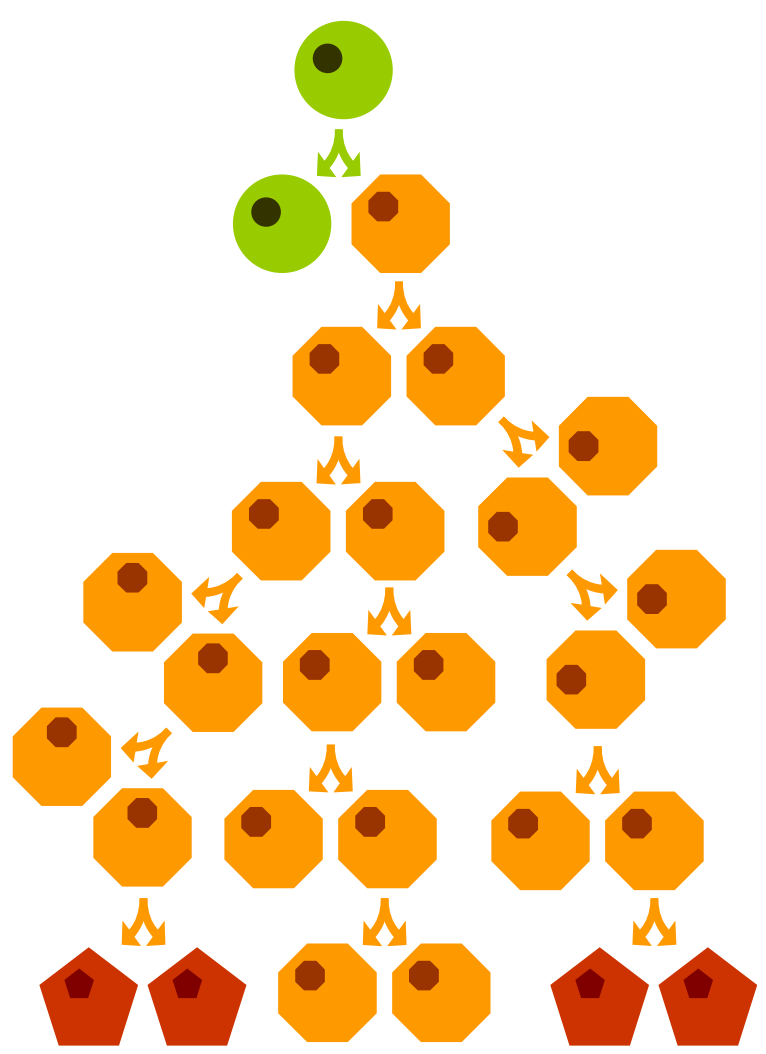

Figure 3. Transit amplifying cells

One stem cell division results in many differentiated cells via transit amplifying cells. The stem cells are green circles, the progenitor, transit amplifying cells are orange octagons, and the differentiated cells are red pentagons.

\subsubsection{Dedifferentiation, redifferentiation and transdifferentiation}

Differentiation might not entirely be a one-way street. Some differentiated cells can dedifferentiate into a less mature phenotype (Figure 4). Chondrocytes for example, when removed form their extracellular matrix and cultured in vitro on tissue culture plastic, will loose their cartilage phenotype. They stop expressing the cartilage-specific marker collagen type II and change morphology from a rounded chondrocyte to a stretched fibroblast-like cell [2]. When growth factors, for example TGF $\beta$ (transforming growth factor), are added to the culture medium, they will redifferentiate into chondrocytes and start expressing collagen type II again.

Transdifferentiation is a switch of a differentiated cell into another differentiated cell, either within the same, or into a completely different tissue (Figure 4). Transdifferentiation does not necessarily involve dedifferentiation and redifferentiation. When the switch of gene expression happens 
quickly, there will be coexistence of markers from both cell types for a short time. Transdifferentiation can be induced by modifying the gene expression of cells. An example of induced transdifferentiation in mammals is the conversion of pancreatic cells to hepatocytes (reviewed by [3]). Whether or not transdifferentiation occurs in vivo is still controversial.
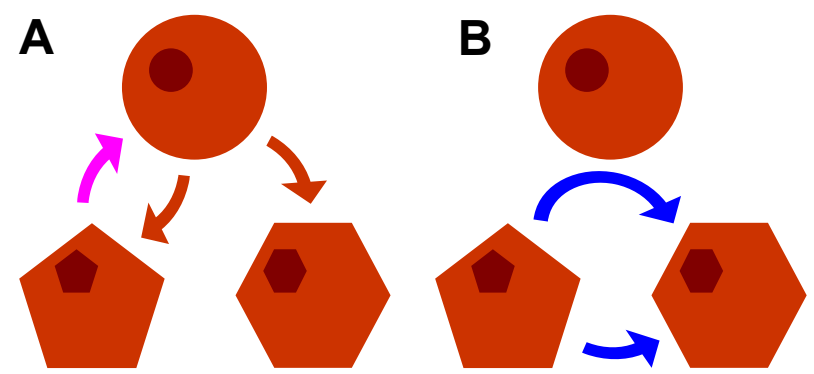

Figure 4. Dedifferentiation, redifferentiation and transdifferentiation

A) A differentiated cell dedifferentiates (pink arrow), and redifferentiates (red arrows) into the same phenotype (pentagon) or a different phenotype (hexagon). B) A differentiated cells transdifferentiates (blue arrow) into another differentiated phenotype, sometimes via an intermediate cell type (circle).

\subsubsection{Plasticity of stem cells}

For many years, researchers thought that adult stem cells could only generate cells of the tissue in which they reside. However, experiments in the past 10 years have shown that adult stem cells may be capable of differentiating across tissue lineage boundaries, sometimes even across germ layers (Figure 5 and 6A). This is called plasticity: the ability of adult stem cells from one tissue to generate the specialized cell type of another tissue. For example, hematopoietic stem cells might contribute not only to the formation of blood cells, but also to the formation of for example skin, liver, brain and heart. Some studies claim the contribution of brain and muscle-derived stem cells to the formation of blood cells.

Recent literature on stem cell plasticity demonstrates a substantial amount of papers dealing with the controversy that surrounds plasticity. There are many possible explanations for the apparent plasticity of adult stem cells (reviewed in [5]). The stem cell population used for experiments might not be homogeneous (Figure 6B). In experiments performed with such heterogeneous cell populations, distinct cell types could contribute to the observed outcome. Many experiments were performed with bone marrow-derived cells, a population known to be very heterogeneous, even after some purification steps. Ideally, the experiments should be performed with clonally derived stem cells. The stem cell populations, although isolated from one tissue, might also contain circulating stem cells. Hematopoietic stem cells, for example, can circulate in the blood, thereby contaminating many non-hematopoietic tissues. 


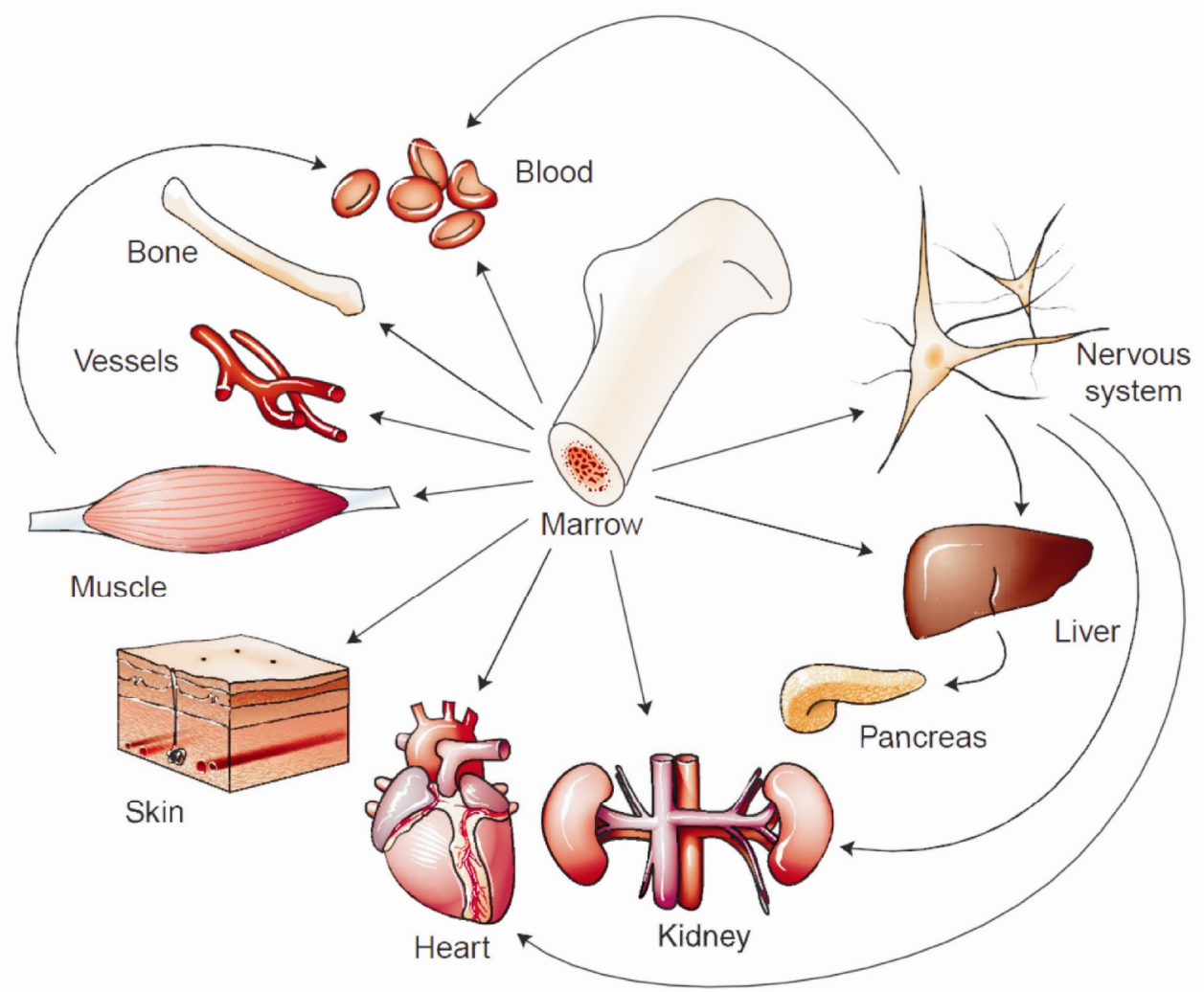

Figure 5. Adult stem cell plasticity: Too good to be true?

Studies in mice yielded evidence, now being reassessed, that stem cells from a variety of tissues can produce progeny in different organs. Bone marrow, which has several types of stem cells, seems particularly versatile. Illustration: C. Slayden [4].

Another explanation of plasticity might be cell fusion. The resulting cells are tetraploid hybrid cells (Figure 6C). Spontaneously fused bone marrow cells can subsequently adopt the phenotype of the recipient cells, which, without detailed genetic analysis, might be interpreted as plasticity $[6,7]$. Others claim that in some tissues, such as liver and muscle, cell fusion is a natural process. Whether this fusion process results in functional tissue cells is, however, still unclear.

Technical problems might also account for some of the plasticity claims. Many of these experiments have been performed with Green Fluorescent Protein-labeled (GFP) stem cells. However, skeletal muscle fibers for example exhibit autofluorescence, resembling the GFP signal. Consequently, in experiments where GFP-labeled stem cells were analysed for their plasticity, the fluorescent signal might not have come from apparently transdifferentiated stem cells, but from the autofluorescing muscle fibers [8] (Figure 6D). 


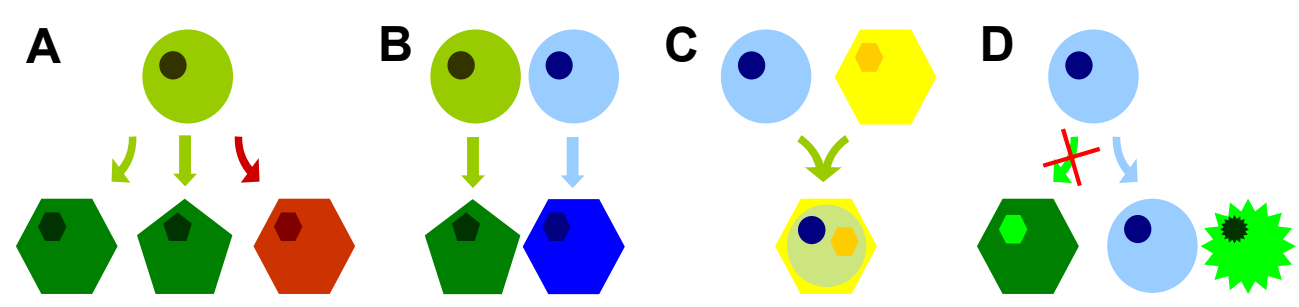

Figure 6. Plasticity of adult stem cells (A) and possible explanations for apparent plasticity (B-D)

A) An adult stem cell from one tissue (light green), cannot only form differentiated progeny of its own tissue (dark green), but also of another tissue (red, on the right). B) A heterogeneous population of (stem) cells (light green and blue) results in diverse differentiated cells (dark green and blue). C) Fusion of a stem cell (blue) with a differentiated cell (yellow) results in a hybrid cell displaying a differentiated phenotype. D) True plasticity would result in differentiation outside the stem cells own tissue, as indicated by GFP signal of the differentiated cell on the left (green nucleus). The actual signal might be background staining of autofluorescent neighboring cells on the right.

\subsubsection{Differentiation of stem cells in vitro}

It is one challenge to keep stem cells undifferentiated in culture; it is another challenge to differentiate the cells into the desired tissue. A major challenge is differentiating the pluripotent or multipotent stem cells into a homogeneous population of cells. Directed differentiation of stem cells in vitro typically involves changing the culture medium. Addition of growth factors, cytokines, or other proteins to the culture medium can induce differentiation into a specific lineage. This involves cell signaling and transcriptional responses. Another option is changing the culture environment of a cell, for example culturing in 3D pellets instead of 2D adherent cultures. Stem cells can also be cocultured with cells of the differentiated phenotype, either in direct contact, in a trans-well system through which only medium components and no cells can diffuse, or by adding conditioned medium of those differentiated cells.

\subsubsection{Epigenetics and differentiation}

All differentiated cells originate from the same fertilized egg, and thus contain the same genetic material. However, the cell morphology and function changes dramatically during differentiation. Growth factors that can stimulate stem cells to differentiate into the neuronal lineage cannot stimulate heart cells to become neurons. The diversity in cell types is caused by differential gene expression patterns. During differentiation, stem cell self-renewal genes have to be silenced, and only the tissue-specific genes have to be transcribed. Upon cell division, this expression pattern has to be passed onto the daughter cells. This stable change in gene expression is coordinated by epigenetic mechanisms. Epigenetics, an emerging field of research, may be defined as the stable alterations in gene expression potential that arise during development (differentiation) and cell proliferation, without altering the DNA sequence. This is regulated through the chromatin structure 
by different mechanisms. Two major epigenetic mechanisms are DNA methylation and histone modifications.

DNA can be covalently modified through methylation, primarily on cytosines of the dinucleotide sequence $\mathrm{CpG}$. Regions of the genome that have a high density of $\mathrm{CpGs}$ are called $\mathrm{CpG}$ islands. Many tissue-specific promoter regions contain $\mathrm{CPG}$ islands, and the hypermethylation of promoterassociated $\mathrm{CpG}$ islands suppresses gene expression. The methylation patterns are passed on to daughter cells during cell division, by the action of DNA methyltransferases.

Chromatin is the structure of genomic DNA of eukaryotic cells that is compacted on nucleosomes. Nucleosomes consist of a histone octamer containing two of each of the histones H2A, H2B, H3 and $\mathrm{H} 4$ around which the DNA is wound. Post-translational modification of histone proteins at their $\mathrm{N}$ terminal 'tail' include methylation, acetylation, ubiquitylation, sumoylation, phosphorylation and addition of ADP-ribosyl groups (Figure 7).

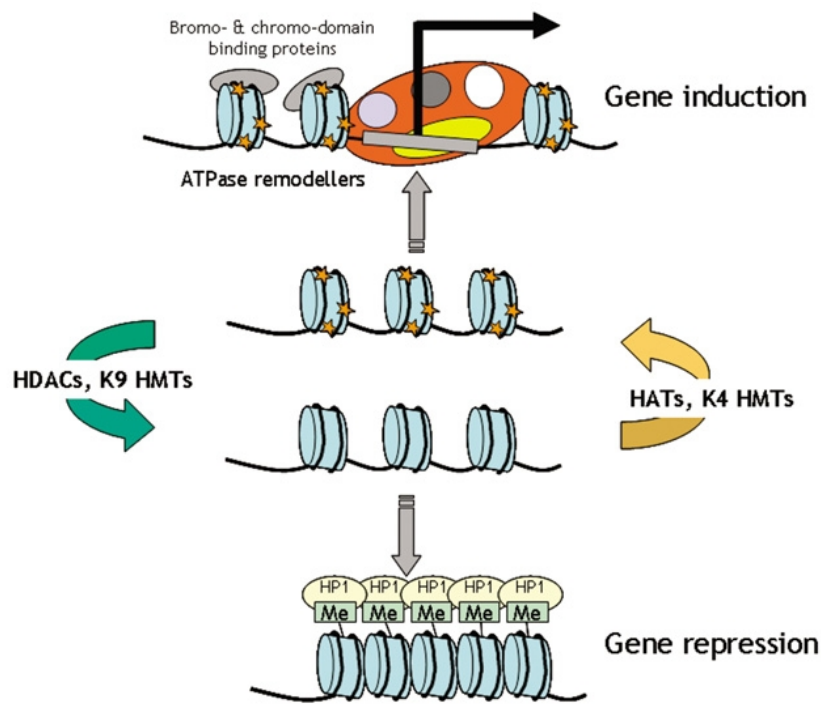

Figure 7. The histone switch

Targeted modifications under the control of histone methylases (HMTs), histone acetyltransferases (HATs) and histone deacetylases (HDACs) alter the histone code at gene regulatory regions. This establishes a structure that contains bromo- and chromo-domains that permits recruitment of ATP-dependent chromatin remodelling factors to open promoters and allow further recruitment of the basal transcription machinery. Deacetylation, frequently followed by histone methylation, establishes a base for highly repressive structures, such as heterochromatin. Acetylated histone tails are shown as yellow stars. Methylation (Me) is shown to recruit heterochromatin protein 1 (HP-1) [9]. 
These modifications can influence chromatin compaction and accessibility for transcriptional complexes. More condensed chromatin, marked by histone methylation, is less accessible for gene transcription. In stem cells, chromatin is less compacted, marked by acetylated histones, than in differentiated cells. Differentiation is accompanied by a successive restriction in the repertoire of genes that can be expressed. This implies a close relationship between differentiation potential and chromatin remodeling.

Epigenetics also plays a role in the maintenance of pluripotency. Recent research suggests that cell fate can be reset by epigenetic reprogramming. By demethylating DNA, for example demethylation of the oct4 promoter region, cells can regain a pluripotent phenotype. The chromatin can be remodeled to be more accessible for gene transcription. There is growing evidence that epigenetic modifications are the core machinery required for nuclear reprogramming and cell-fate conversion. These remarkable findings suggest that epigenetics provide an important new research field for improving regenerative medicine. An example of epigenetic reprogramming is given in section 5 ( State of the Art Experiment).

\section{Embryonic stem cells}

Embryonic stem cells do not exist in the body. When cells are isolated from the inner cell mass (ICM) of the blastocyst, they can be massively expanded in the laboratory, while maintaining their pluripotency (self-renewal). These in vitro propagated cells are called ESCs. Mouse ESCs were the first to be isolated $[10,11]$. The next major breakthrough was in 1998, when Thompson et al. isolated ESCs from human embryos [12].

\subsection{Isolation of embryonic stem cells}

Mouse ESCs can be isolated from super-ovulated or naturally mated females. After 3.5 days, the pregnant mice are sacrificed and the blastocyst stage embryos are flushed from the uterine horn. The blastocyst contains the trophectoderm (the outer layer of cells), a fluid filled cavity called the blastocoel, and an ICM. The embryos are transferred to a culture dish, and after attachment, the ICM can be isolated from the rest of the embryo by aspirating it into a pipette. The ICM is then transferred to a new dish, and examined for undifferentiated morphology (Figure 8).

The cell colonies that grow from these cells have to be dissociated every few days, to prevent differentiation of the ESCs. This is usually done by the addition of trypsin, an enzyme that dissociates cells from each other and from the plastic of the culture dish. The single cells will form new colonies, and some of these colonies will remain undifferentiated. 


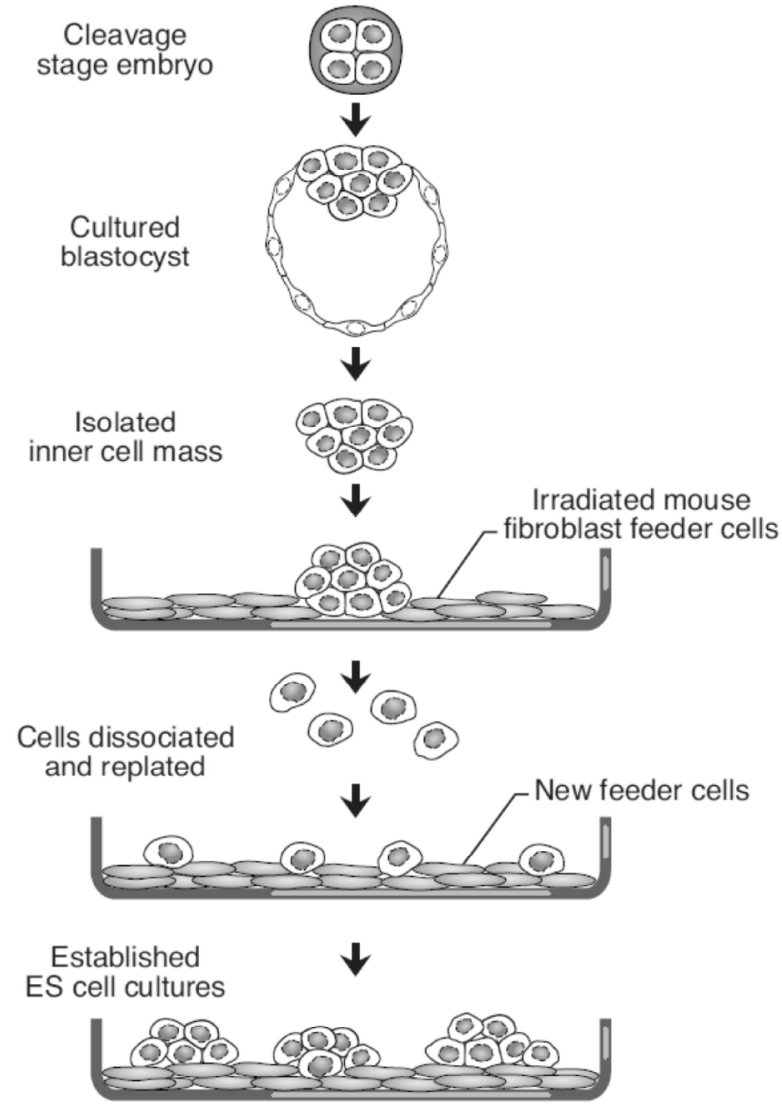

Figure 8. Derivation of human ESC lines

Human blastocysts were grown from cleavage-stage embryos produced by in vitro fertilization. ICM cells were separated from trophectoderm by immunosurgery, plated onto a fibroblast feeder substratum in medium containing fetal calf serum [14].

To keep mouse ESCs undifferentiated, they have to be grown in optimal conditions. The mouse ESCS attach and grow on a feeder layer prepared from mouse embryonic fibroblasts, which are mitotically inactivated, either by irradiation, or treatment with the toxic antibiotic mitomycin-C. The feeders cells do not replicate, but they do produce mostly unknown factors that keeps the ESCS undifferentiated. The discovery of Leukemia Inhibitory Factor (LIF) in 1988 [13], allowed researcher to grow mouse ESC in the absence of a feeder layer.

Flushing the ovary ducts of a pregnant woman is not an option for the isolation of human ESCs. Therefore, surplus embryos of IVF treatment that are donated after informed consent of the parents are used. First an oocyte is fertilized in vitro by a sperm cell. The zygote, the fertilized egg, is grown in vitro until it reaches the blastocyst stage. Instead of being transferred to the uterus, these 5-day 
old blastocysts are used to isolate human ESCs. The blastocysts contain approximately 200-250 cells, of which 30-34 cells form the ICM. The trophectoderm can be removed by mechanical surgery (cutting with a small scalpel) or immunosurgery (antibodies break down the trophectoderm).

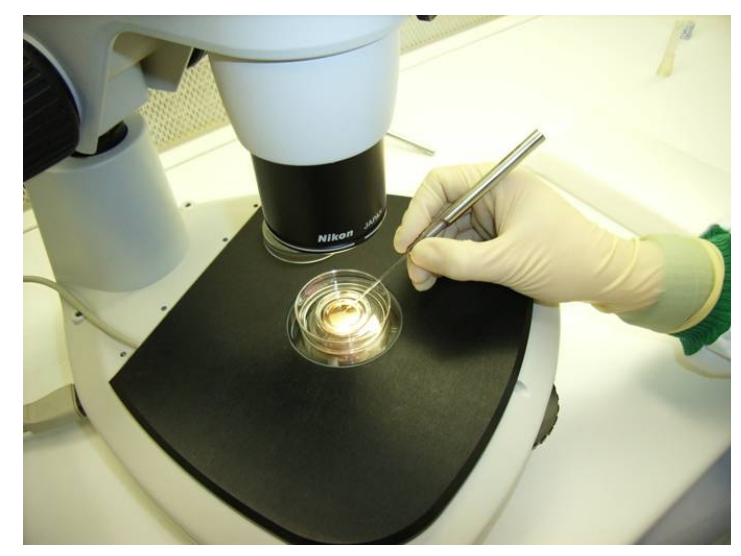

Figure 9. Human ESC colony transfer

Colonies are cut into pieces with a cutting pipette made from a glass capillary and transferred to a dish with new feeder cells.

The ICM is cultured on a feeder layer, similar to the isolation of mouse ESCs. However, LIF cannot keep human ESCS undifferentiated. Human ESCS have to be cultured on a feeder layer in the presence of serum or serum-replacement in combination with basic fibroblast growth factor (bFGF). Several human ESC lines cannot be dissociated by the use of trypsin. Therefore, these colonies are mechanically dissected by cutting them in pieces with a knife made of a glass capillary (Figure 9). The colony pieces are then transferred to a new dish with feeder cells.

ESCs are very sensitive to temperature and $\mathrm{pH}$ change, and when colonies overgrow, they also tend to differentiate. Therefore, ESCs have to be cared for every day, also in the weekend and during holidays.

\subsection{Characterization of embryonic stem cells}

The derived ESC lines will be cultured for months, to ensure their self-renewal capacity. Human ESCS have been reported to proliferate for years and go through hundreds of population doublings [15].

There are some markers that can be used to determine the undifferentiated state of ESCs. The best characterized is Oct4. Undifferentiated cells express the Pouff1 gene, which encodes for the transcription factor Oct4. Loss of pluripotency of ESCS is often accompanied by a down regulation of Oct4 expression. Other markers are the enzyme alkaline phosphatase (ALP), stage-specific 
embryonic antigen (SSEA)-1 for mouse ESCs, and SSEA-3 and SSEA-4 for human ESCs, and tumor rejection antigen TRA1-60 and TRA1-81 for human ESCs. ESCs also express high levels of telomerase.

The pluripotency of the ESCS can be identified both in vitro and in vivo. In vitro differentiation generally starts with the formation of embryoid bodies (EBs): free-floating aggregates of randomly differentiating cells (Figure 10). When ESCs are placed in a non-adherent bacterial dish or in small droplets hanging from a bacterial lid (hanging drop method), they will spontaneously form cell aggregates in which cells start differentiating in a fashion that resembles early post-implantation embryos. Cell types of all the three germ layers (ectoderm, mesoderm and endoderm) are formed. Once the EBs are allowed to attach to a culture dish, differentiated cells will grow out of the aggregates. These can be identified by morphology (for example spontaneously contracting cardiac muscle cells), or immunostaining for specific cell types.
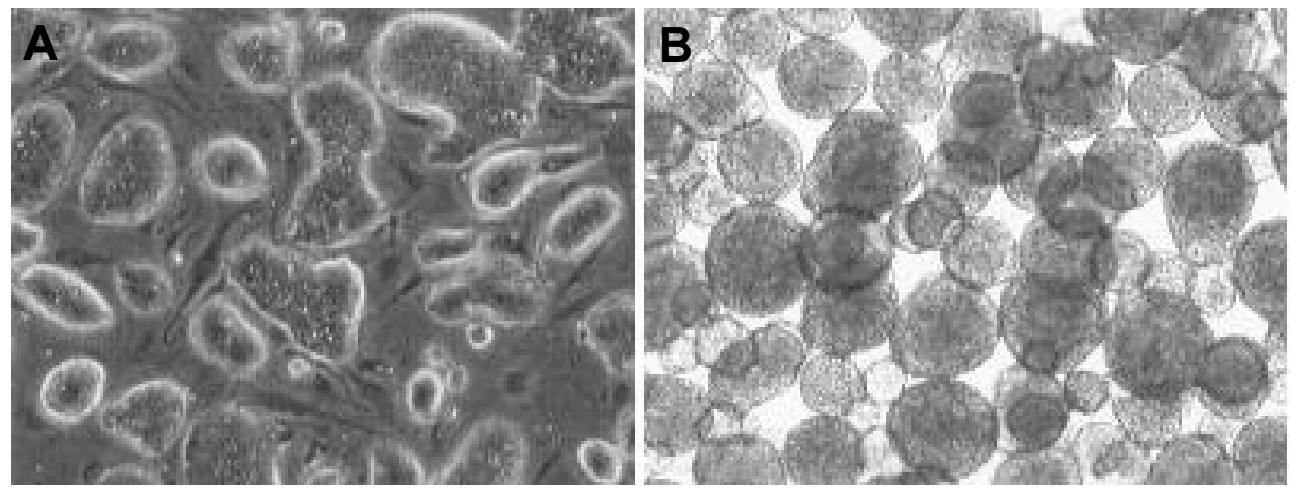

Figure 10. The onset of ESC differentiation

A) Mouse ESCs in colonies attached to culture plastic. B) Embryoid bodies floating in the culture medium.

An in vivo method for determining the pluripotency of ESCs is the injection of ESCs under the skin or in the kidney or testis of an immuno-deficient mouse. A benign tumor, called teratoma, will form and advanced tissue types of all three germ layers can be identified (Figure 11), for example gut epithelium (endodermal), cartilage and bone (mesodermal) and neural tissue (ectodermal).

The ultimate proof of pluripotency of mouse ESCS is the formation of chimeric mice, in which the cells have contributed to the formation of all tissues, including germ cells. This has only been achieved for mouse ESCs. First, the researcher has to test whether the number of chromosomes is normal and whether the chromosomes are not damaged. This can be done by karyotyping. Next, an ESC can be injected into the cavity of a blastocyst, and transferred to the uterus of a pseudopregnant mouse. The offspring are chimeric mice, of which all tissues are composed partly of host cells and partly of the donor ESCs (Figure 12). 

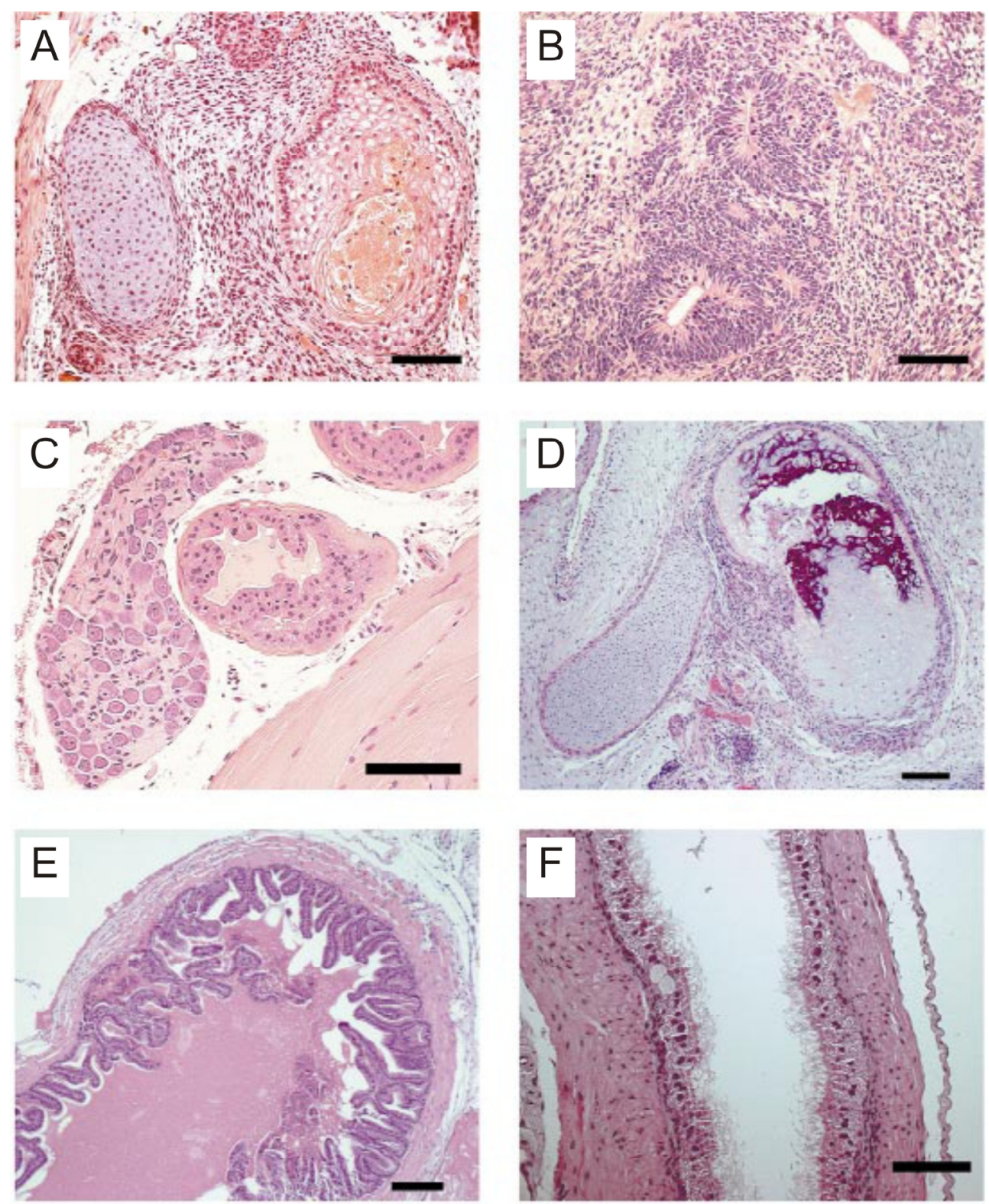

Figure 11. Histology of differentiated elements found in teratomas formed in the testis of immunedeficient SCID mice following inoculation of two human ESC colonies (HES-1 and HES-2)

A) Cartilage and squamous epithelium, HES-2. B) Neural rosettes, HES-2. C) Ganglion, gland, and striated muscle, HES-1. D) Bone and cartilage, HES-1. E) Glandular epithelium, HES-1. F) Ciliated columnar epithelium, HES-1. Scale bars: (A-E) $100 \mu \mathrm{m}$; (F) $50 \mu \mathrm{m}[16]$. 

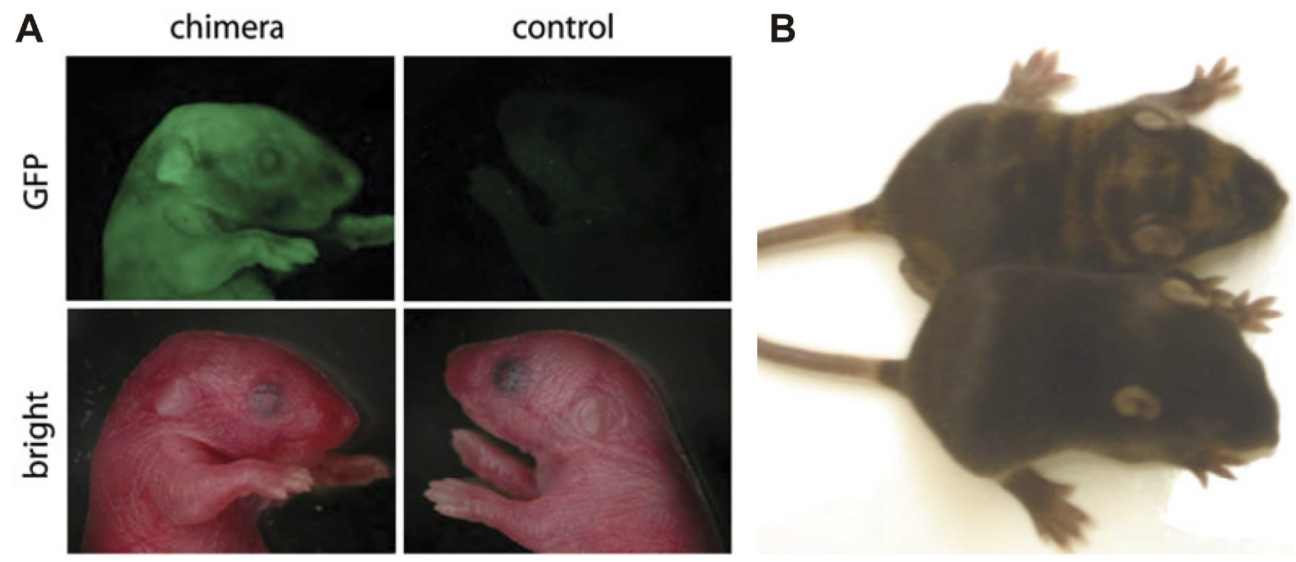

Figure 12. Chimeric mice from ESC-like cells

ESC-like cells are induced pluripotent stem cells as described in section 5 State of the Art experiment [17]. A) Cells from iPS line 2D4 that carried a randomly integrated GFP transgene were injected into blastocysts. Surrogate mothers gave birth to GFP-positive pups. A nonchimeric pup not expressing GFP is shown. B) Ten-dayold chimeric mouse derived from blastocyst-injected 2D4 iPS cells, shown next to a wild-type littermate. iPSderived cells are responsible for the agouti coat color.

\subsection{Self-renewal of embryonic stem cells}

\subsubsection{Self-renewal of mouse embryonic stem cells}

Cytokines or growth factors have to be added to the culture medium to keep mouse ESCS undifferentiated. One signaling pathway involved in the self-renewal of mouse ESCs is the LIF-STAT3 pathway. LIF binds to a receptor complex of the LIF and gp130 receptor, which triggers the activation of the transcription factor STAT3 (Signal Transducer and Activators of Transcription). LIF cannot support self-renewal in the absence of serum, indicating that its activity is dependant on one or more factors that are present in the serum. A possible candidate is BMP4 (bone morphogenetic protein 4), which induces the expression of transcription factors of the Id (inhibitors of differentiation)-family [18]. These transcription factors block differentiation.

One of the master genes of mouse ESC pluripotency is the transcription factor Nanog $[19,20]$. Nanog is named after Tir Na Nog, Land of Ever Young or Land of Eternal Youth in Irish mythology. In vivo, Nanog is expressed in the morula. In the blastocyst stage, expression is limited to the ICM. In vitro, Nanog is enriched in undifferentiated ESCs, but is downregulated in differentiating ESCs and in adult tissues. In mouse ESCs, high levels of Nanog can maintain pluripotency in the absence of LIF, and Nanog enables human ESCs to grow undifferentiated in the absence of feeders. 
Overexpression of Nanog does not seem to affect phosphorylated STAT3 levels, nor does elevated STAT3 signaling result in changed Nanog expression. Despite all this, a functional STAT3 binding site is present in the Nanog promoter region. How Nanog and STAT3 signaling cooperate in the maintenance of pluripotency is still largely unclear.

In contrast, a direct interaction between Nanog and BMP4 signaling has been described. BMP4 signaling is mediated via the activation of SMAD1. Nanog can interact with SMAD1, and thereby inhibits the actions of BMP. Since BMPs are also involved in mesodermal differentiation, the inactivation of the BMPs by Nanog can help in maintaining the undifferentiated state of ESCs. The expression of Nanog is regulated by a number of transcription factors (Figure 13).

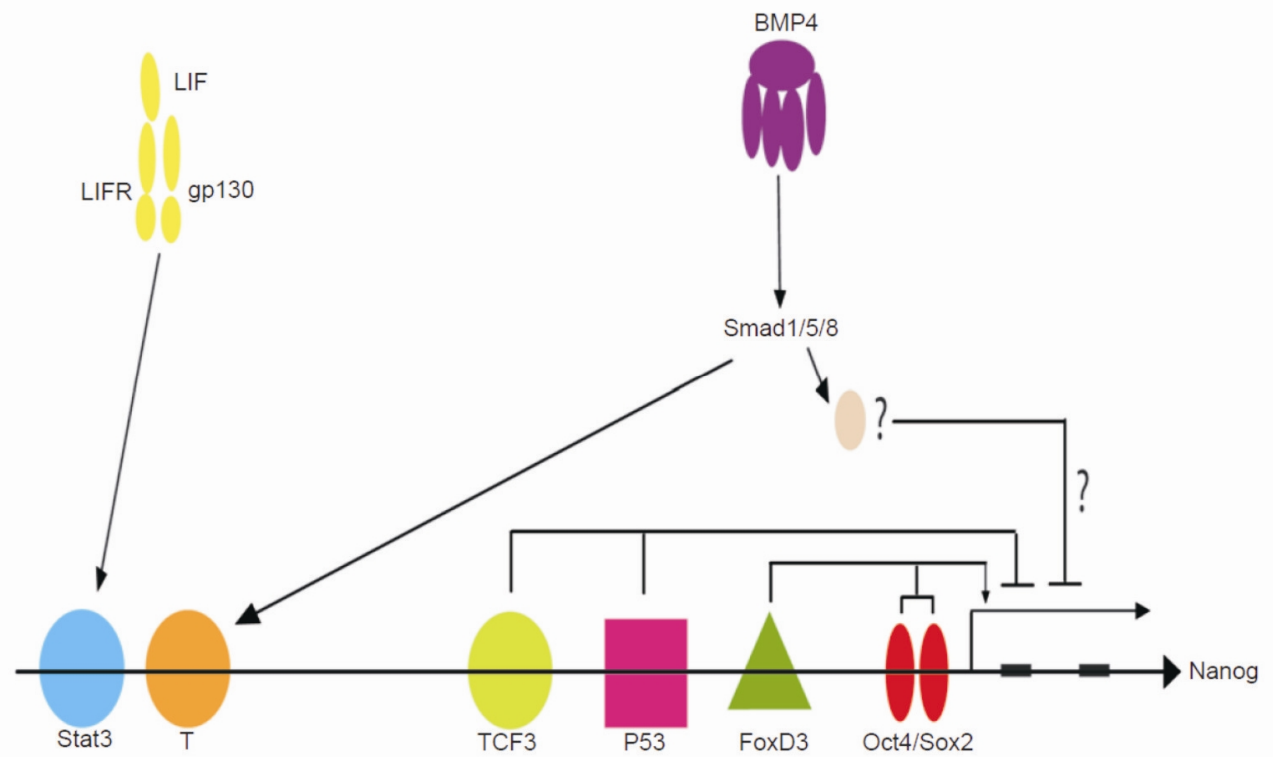

Figure 13. Regulation of Nanog expression

FoxD3 and Oct4/Sox2 bind to the proximal region of Nanog promoter and support its expression. TCF3 and p53 also bind to the promoter and negatively regulate Nanog expression. LIF and BMP signaling and their downstream effectors STAT3 and T may also be involved in Nanog regulation [21].

The most important are Oct4 and Sox2, which can bind to an Oct4/Sox2 motif in the Nanog promoter, thereby activating Nanog transcription. However, in oct4-deficient mice, Nanog is still expressed. Therefore, other factors must also be involved in regulating Nanog expression. FoxD3, a transcription factor of the forkhead family, is highly expressed in ESCs and can bind to an enhancer in the Nanog promoter, thereby activating gene transcription. Interestingly, Nanog can also positively autoregulate its own expression. 
To allow differentiation during embryonic development, Nanog must also be negatively regulated. The tumor suppressor p53 and Tcf3, a transcription factor in the Wnt pathway, are considered to be negative regulators of Nanog expression in ESCs.

As for Nanog, other key transcription factors involved in maintaining pluripotency, i.e. Oct4, Sox2 and FoxD3, can positively autoregulate their expression. In addition, they also bind to each others promoter region, thus forming a negative feedback loop. Nanog, Oct4 and Sox2 cooperatively regulate the expression of many target genes. In conclusion, several key transcription factors involved in maintenance of pluripotency activate or inhibit each other's expression and they simultaneously regulate a set of target genes. These cooperative actions result in the formation of a regulatory network, that balances the maintenance of self-renewal and the ability of stem cell differentiation (reviewed in [21]).

\subsubsection{Self-renewal of human embryonic stem cells}

One might expect that mouse and human ESCs self-renewal are regulated by similar mechanisms. However, it appears to be regulated by many different pathways. The LIF/STAT pathway fails to maintain self-renewal of human ESCs [22] and BMP4 seems to stimulate differentiation of human ESCs. Apparently, the feeder cells produce other unknown factors to support the self-renewal of human ESCs. TGFß family signaling, and especially the TGFß/Activin/Nodal pathway, plays a role. Activin A may be one of the critical factors produced by the feeder cells. Prolonged culture in the absence of feeders is possible in the presence of TGFß1, bFGF and LIF [23]. It is well-known that bFGF can maintain the pluripotency of human ESCs (Figure 14) but the detailed mechanism is still unclear. Many other pathways have been associated with the self-renewal of human ESCs, but there appear to be differences between individual ESC lines and culture conditions (feeder or feeder-free cultures on extracellular matrix products such as laminin or Matrigel).

The transcriptional regulatory network involved in self-renewal is being elucidated piece by piece. Oct4 is expressed by mouse and human ESCs, but in itself is insufficient to maintain self-renewal. Nanog expression is high in human ESCs, and is reduced in differentiating cells. Some similarity between the mechanism through which Nanog regulates the maintenance of pluripotency in mouse and human ESCs has been identified, but the exact regulatory network is not clear yet. 

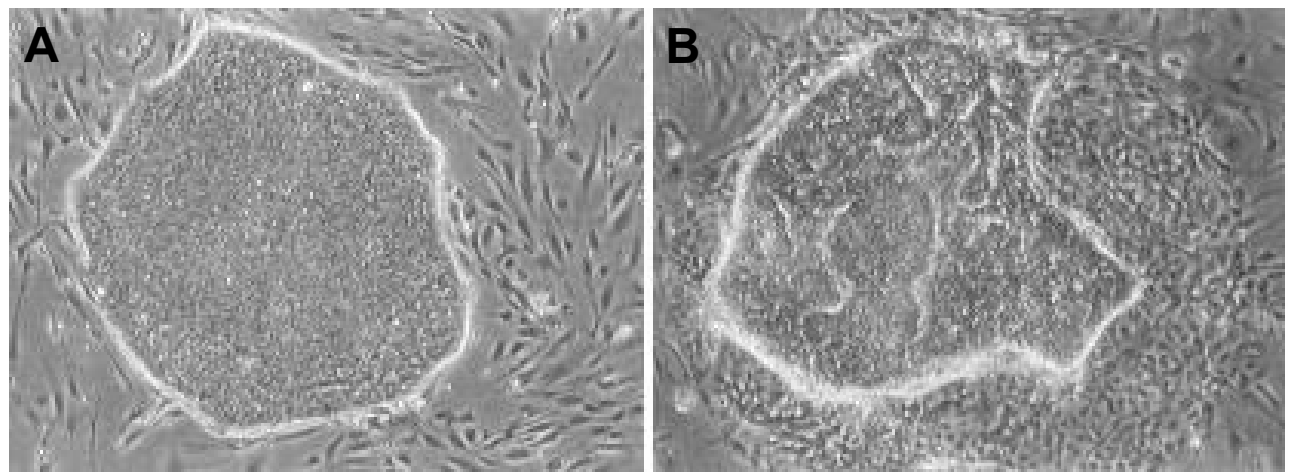

Figure 14. Human ESC colonies on feeder cells

A) This colony displays successful self-renewal. B) This colony contains many differentiated cells, as can be seen from the heterogenic pattern of the colony and the outgrowth of cells.

\subsection{Differentiation of human embryonic stem cells}

Human ESCs might be an ideal cell source for tissue engineering and regenerative medicine, because of their indefinite proliferation capacity and pluripotency. ESCs are often mentioned as a promise for the cure of Parkinson's disease, diabetes and cardiovascular diseases. This inevitably means that human ESCs have to be differentiated into respectively neurons, insulin-producing cell and cardiomyocytes. Indeed, many articles are published in which the in vitro and in vivo differentiation of human ESCs is described. Useful cell types such as neurons [24-26], cardiomyocytes [27,28], hepatocytes [29,30], pancreatic beta cells [31], endothelial cells [32], blood cells [33,34] and chondrocytes [35] have all been successfully derived in the laboratory. Levenberg and co-workers demonstrated the differentiation of human ESCs on polymeric scaffolds into 3D structures with characteristics of developing neural tissues, cartilage, liver, or blood vessels [36].

\subsection{Application of embryonic stem cells}

\subsubsection{Application of mouse embryonic stem cells}

It is obvious that mouse ESCs will never find clinical applications. However, the knowledge of mouse ESCs was used for the isolation, growth and differentiation and thus possible future application of human ESCs. Furthermore, mouse ESCs are used as a model system to study early embryonic development and differentiation.

Another valuable feature of mouse ESCs is the ability to create genetically modified mice. When genes are introduced into an ESC, and these ESCs contribute to the germ cells (eggs or sperm) of the chimeric progeny, it is possible to breed a line of genetically changed mice. In a knock-out mouse, the function of a gene is disturbed, and the biological function of the gene can be studied. When a 
mutation is introduced that is known to be the cause of a human genetic disease, the mice may serve as a model for this human disease. Much of the knowledge of stem cell self-renewal is also based on the use of these knock-out, knock-in and gene over-expression models.

\subsubsection{Application of human embryonic stem cells}

The first step that has to be taken before it is feasible to use human ESCs in therapeutic application is the optimization of the culture method. The current method of colony growth on feeder cells is time-consuming and labor-intensive, and large-scale propagation of these cells is not possible. Feeder-free growth of enzymatically passaged human ESCs would be a huge step forward.

Another optimization of the culture protocol would be the derivation and growth under animal product-free conditions. After the discovery of prion diseases, such as Creutzfeldt Jakob (Bovine Spongiform Encephalopathy or mad cow disease), the risk of infection by non-human pathogens is well-recognized. It is not just the animal sera used in culture that contaminate the human ESC cultures. In 2005, it was discovered that human ESCs take up non-human sialic Neu5Gc [37] from the growth media and feeder cells. This uptake and expression of Neu5Gc on the surface of any tissue developed from human ESCs could induce an immune response upon transplantation. The use of serum-replacement media instead of serum in the media does not solve this problem, since there are still animal proteins in the serum replacement. The extracellular matrix proteins that may replace the feeder cells are usually from animal sources. Thus, the only solution would be to culture on human feeders, using human serum and supplements.

Immune-rejection can be expected from any human ESC line that is not derived from the patient's own cells. A patient would have to take immuno-suppressive drugs for the rest of his life. ESC could be genetically modified to escape host immune responses. Alternatively, one could make patientspecific ESCs. A first step in that direction is given in section 5 (State of the Art experiment).

Another challenge will be the purification of the cell type of interest out of heterogeneous cell populations. The complex signaling pathways and growth factor requirements to differentiate ESCS into one cell type are not fully understood. As a result, even though specific growth factors are added to the culture medium, the outcome is usually a low proportion of differentiated cells in a mixed population of cells. Purification of a cell population can be done by selecting for tissue-specific surface markers, or by transducing the cells using a tissue-specific promoter to drive a selectable 
marker such as antibiotic resistance. In the latter way, only cells of the desired tissue type will survive treatment with antibiotics.

Besides selecting for the differentiated cells, it will also be important to negatively select for the remaining undifferentiated population of ESCs. These cells, once implanted into the patient, may have the capacity to form teratomas. Although these tumors are generally benign, it is a highly undesired side-effect.

Another major milestone will be the demonstration of treatment efficacy in large animal models. ESCs have been isolated from rhesus monkey [38], cynomolgus monkey [39] and marmoset [40], which are widely used as experimental animal models for human diseases. These ESC lines could be a useful resource for preclinical stem cell research and developing ESC-based transplantation therapies.

In conclusion, in the past 10 years there has been tremendous progress in the isolation procedures and differentiation of human ESCs, but therapeutic application is still premature.

\subsection{Political and ethical concerns}

ESC research is controversial because of the use of human embryos. One of the main questions in ESC isolation is whether destroying the embryo means destroying life. To answer that question, one has to define when life begins. For some (religions), life starts at fertilization of the egg, for others after 40 days, or after 120 days when the embryo gets a soul, or sometimes even at birth.

In some countries, research on human ESCs is completely forbidden, in other countries research is allowed but it is illegal to create new lines. Not all countries have policies covering ESC research and many countries have a moratorium on ESC research. Law and guidelines generally describe:

- The use of embryos for research

- The production of embryos for research

- The use of left-over embryos from IVF treatment

- The creation of new ESC lines

- The use of already established human ESC lines

- Somatic Cell Nuclear Transfer in ESCs and therapeutic cloning

- Reproductive cloning

In Europe, ESC research is allowed for example in the United Kingdom, the Netherlands, Sweden and Belgium, whereas it is forbidden in Norway and Austria. In Germany, only ESCs that date from 
before 2002 can be imported. Australia and Israel allow ESC isolation from excess IVF embryos. In the United States, ESC research is not forbidden, but is not funded by federal funding. Only cell lines that were created before August 9, 2001 ("where the life and death decisions have already been made") are allowed in federally funded research. These lines are registered at the National Institute of Health, but not all of the 71 lines are of good quality for research. In 2006, President Bush used his veto power for the first time in his presidency, to veto a bill that would allow the government to spend federal money on ESC research that uses embryos left-over from IVF treatments. Privatelyfunded research and isolation of human ESCs is allowed.

\section{Adult stem cells}

Adult stem cells are undifferentiated cells, which reside in differentiated tissues. The first adult stem cells were discovered almost 50 years ago. Researches discovered that bone marrow contained two different populations of stem cells; hematopoietic stem cells (HSCs) [41,42] and bone marrow stromal cells [43]. The HSC give rise to all red and white blood cells and also platelets. The bone marrow stromal cells are a heterogenic population of progenitor cells giving rise to bone, cartilage, fat and haematopoiesis-supporting stroma. After this first discovery, adult stem cells were found in many different tissues such as heart, epidermis, liver, pancreas, brain, dental pulp and spinal cord.

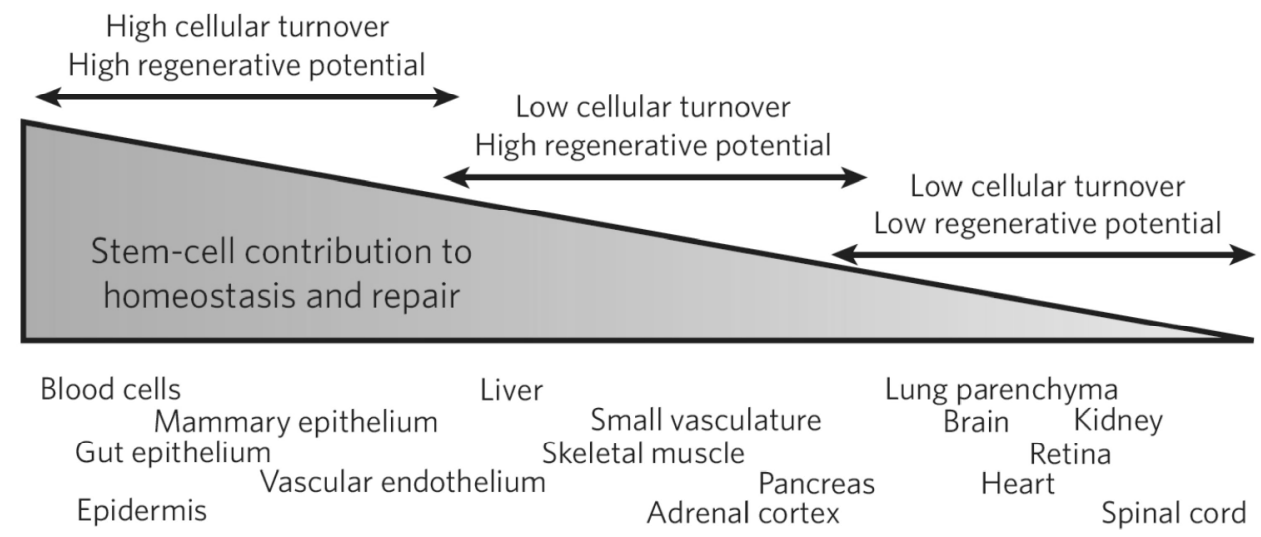

Figure 15. Stem cell contribution to homeostasis and repair [44]

Tissues can be divided into three categories (Figure 15).

- Tissues with high turnover (such as blood, skin and gut) have a prominent stem-cell compartment and have high regenerative capacity. 
- Tissues with low turnover but high regenerative potential. In skeletal muscle, for example, differentiated myofibres are unable to proliferate to generate new tissue, so after injury, muscle must rely on resident stem cells for all turnover and repair.

- Tissues with low turnover and low regenerative potential. Although there is much interest in harnessing the potential of stem cells in the brain and heart for therapeutic purposes, for example, there is limited endogenous repair capacity of these tissues following acute injuries.

\subsection{Isolation of human adult stem cells for research}

Human adult stem cells that are used for tissue engineering research are generally isolated from easily-accessible tissues.

HSCs can be isolated from the bone marrow, but since it is an invasive procedure, this becomes increasingly infrequent. HSCs can also be released in large numbers from the bone marrow compartment into the peripheral blood. This process is called mobilization and is induced by treating the patients with cytokines, such as G-CSF (granulocyte colony stimulating factors). Blood from the placenta and umbilical cord is an alternative rich source for HSCs. It is an appealing cell source, since these tissues are usually discarded after birth.

Mesenchymal stem cells (MSCs) can be harvested from the bone marrow of the iliac crest or femoral heads from patients undergoing total hip replacement. As these patients are already under anesthesia, the harvesting is somewhat less invasive. Recently fat has also been recognized as a rich source for multipotent stem cells. These adipose-derived stem cells are isolated from fat after liposuction.

Most researchers collaborate with academic hospitals to have access to stem cells. Some stem cells, such as MSCs are also commercially available. It is important to realize that cells isolated from one patient might behave differently than cells from another patient. Therefore, research has to be performed with stem cells from multiple patients, in order to validate the obtained results.

\subsection{Characterization of adult stem cells}

Adult stem cells are rare in the human body. The isolation of stem cells generally results in a heterogeneous population of cells. Many cell surface markers have been identified for the various stem cells. Combinations of several markers, or the absence of other markers, allows researchers to enrich or purify the population. Many of these markers are CD molecules (Cluster of Differentiation). Human HSCs are not as well-defined as mouse HSCs yet. CD34 was the first differentiation marker to be recognized and is still the most commonly used marker to obtain enriched populations of human HSCs. This population still contains many other cell types. Other markers include the absence of 
CD38, the presence of CD43, CD45RO, CD45RA, CD59, CD90, CD109, CD117, CD133, CD166 and Lin. A combination of CD34 with one or more of these markers results in a highly purified population. MSCs can be characterized by the presence of STRO-1 (from bone marrow STROmal cells) and the absence of CD34. More markers have been identified, but the isolation of a homogeneous mesenchymal cell population has not been achieved (see the discusion in section 4 Classical Experiment).

For tissue engineers, functional characterization is more important. The potency of a stem cell population to differentiate into various tissues can be analyzed in vitro, but the ultimate proof is formation of functional tissue in vivo.

For HSCs, the best described in vivo assay is the long-term repopulation assay. First, a mouse receives a dose of irradiation sufficient to kill its blood producing cells. Next, HSCs are injected into this lethally irradiated mouse. When the mouse recovers, and the injected cells have repopulated the entire haematopoietic system, these cells can be retransplanted into the next lethally irradiated mouse. When this mouse recovers as well, these cells are considered long-term stem cells capable of self-renewal.

For human MSCs, the in vitro formation of adipose tissue, cartilage, and mineralization is described in section 4 (Classical Experiment). However, mineralization in vitro is not a proof of functional bone formation. Therefore, MSCs will have to be implanted and analyzed for bone formation $[45,46]$. In vivo experiments with human MSCs can only be performed in immuno-deficient animals (mice and rats) and are mostly performed ectopically (not in bone). Orthotopic implantations of MSCs in large bone defects cannot be performed in humans. Therefore, researchers use large animal models, like the goat, to analyse the bone forming capacity of goat MSCs. Similarly, when bone marrow stromal cells are induced into the myogenic lineage, the cells do not only show characteristics of muscle cells in vitro. Upon transplantation, they also differentiate into muscle fibers [47].

\subsection{Self-renewal of adult stem cells}

Developmental signaling pathways such as Notch, Wnt and Hedgehog signaling are involved in the self-renewal of many adult stem cells, all in a context-dependent manner. Besides these extrinsic factors, self-renewal is also regulated intrinsically.

Stem cell self-renewal is regulated through the chromatin structure. Polycomb group proteins (PcG) can repress transcription of genes linked to differentiation by regulating chromatin structure. In particular Bmi-1, Mel-18 and Rae-28 (members of the Polycomb repression complex 1) are involved 
in the maintenance of self-renewal in HSCs. Overexpression of Bmi-1 promotes HSC self-renewal, by enhancing symmetrical cell divisions. Bmi-1-deficient cells have increased expression of the cell cycle inhibitor $\mathrm{p} 16^{\mathrm{lnk4a}}$, which results in senescence and increased expression of $\mathrm{p} 19^{\mathrm{Arf}}$, which is linked to apoptosis. Thus, the mechanism by which Bmi-1 modulates HSC self-renewal seems to be through stimulation of symmetric cell division and the induction of survival genes and simultaneously the repression of anti-proliferative genes [48].

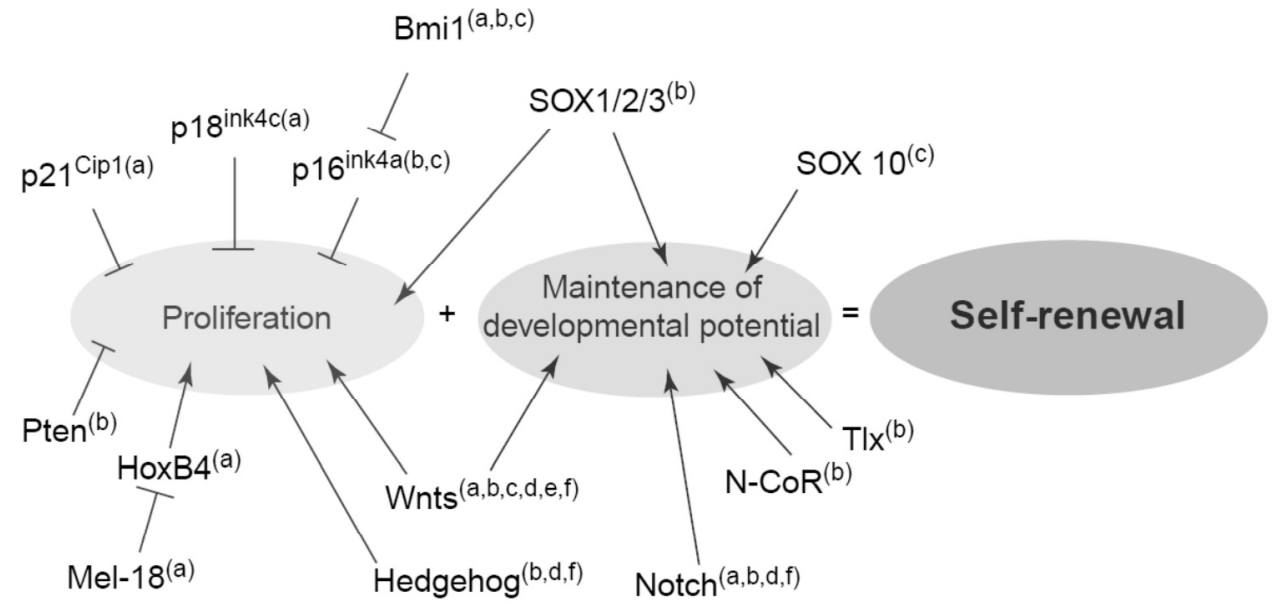
(a) Hematopoietic system
(b) Central nervous system
(c) Peripheral nervous system
(d) Skin
(e) Intestinal epithelium
(f) Germline stem cells

Figure 16. Self-renewal of adult stem cells

Current Opinion in Cell Biology

Regulators of somatic stem cell self-renewal can affect the ability of stem cells to proliferate, retain their developmental potential, or both. The maintenance of developmental potential includes establishing the competence to express each potential fate as well as inhibiting the act of lineage commitment and/or differentiation. It is possible that distinct mechanisms are employed to regulate competence as opposed to the actual decision to commit and/or differentiate. However, the precise mechanisms by which the depicted gene products regulate developmental potential is uncertain. This figure represents recent work in progress as additional regulatory proteins will exist, and the mechanisms by which the depicted proteins regulate selfrenewal will continue to be elucidated [49].

Other genes that are required for self-renewal are thought to negatively regulate differentiation. For example, $t / x$ and $N$-Cor promote self-renewal of neural stem cells by inhibiting the differentiation towards astrocytes. Sox genes are involved in both self-renewal and differentiation of neural stem cells in a time and context dependant manner. 
Furthermore, control of cell cycle and proliferation machinery is required for self-renewal regulation. Deficiency of the early G1 phase regulator $\mathrm{p} 16^{\operatorname{lnk} 4 \mathrm{c}}$ leads to increased HSC self-renewal. The late $\mathrm{G} 1$ phase regulator $\mathrm{p} 21^{\mathrm{cip} 1}$ deficiency leads to increased proliferation and thereby stem cell exhaustion. These mechanisms are summarized in Figure 16 and reviewed in [49]. In conclusion, somatic stem cells have tissue-dependent mechanisms for self-renewal.

\subsection{Stem cell niche}

The idea that stem cells are located in specific anatomical locations in adult tissue called 'niches' was introduced in 1978 by Schofield [50]. The niche is the stem cell microenvironment that provides a sheltering environment for the stem cells, in which the balance between stem cell quiescence and activity is maintained. Stem cells reside in the niche for an indefinite period of time while selfrenewing and producing differentiated progeny. The balanced interaction between the stem cell, the niche cells, the extracellular matrices and secreted factors ensures the maintenance of the stem cell phenotype, and guides a differentiating daughter away from the niche [51].

Many recently characterized niches appear to be simple in structure and operate using common mechanisms. In a simple niche, stem cells are locked to niche cells by adherens junctions and to the extracellular matrix through, amongst others, integrins. The niche positions the stem cells to receive intercellular signals to control growth and inhibit differentiation. (Figure 17A) In a more complex niche, different stem cells might be localized in the same niche, or more cell types contribute to the niche (Figure 17B). In a different type of niche, the storage niche, quiescent stem cells reside. These reserve stem cells are activated in case of wounding and subsequently divide and migrate to contribute to repair injured tissue (Figure 17C).

As a tissue engineer, it is important to realize that a stem cell, once isolated, purified and cultured, is devoid of its niche, and as a result, is not likely to behave as it would in the body. It might be crucial to understand the signals a cell receives when it is located in the niche, to be able to keep cells undifferentiated when placed in culture. 
(a) Simple niche

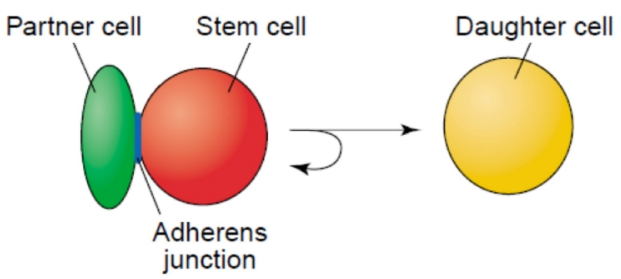

(b) Complex niche

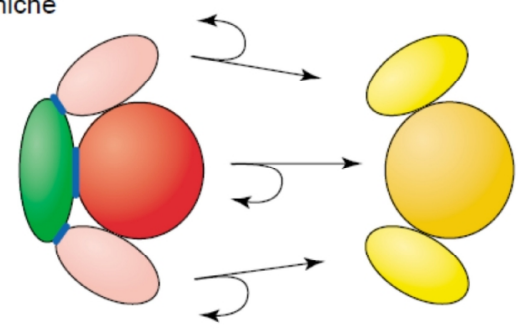

(c) Storage niche

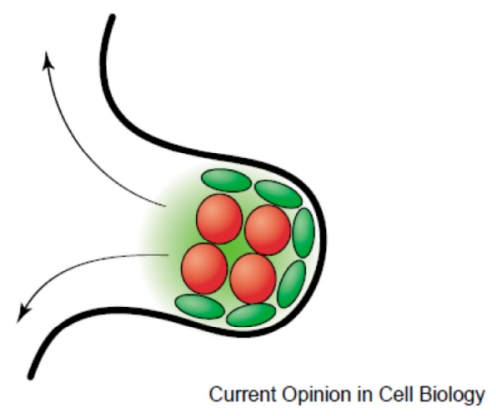

Figure 17. Proposed niche types

A) Simple niche. A stem cell (red) is associated with a permanent partner cell (green) via an adherens junction (blue). The stem cells divides asymmetrically to give rise to another stem cell and a differentiating daughter cell (orange). B) Complex niche. Two (or more) different stem cells (red and pink) are supported by one or more partner cells (green). Their activity is coordinately regulated to generate multiple product cells (orange and yellow) by niche regulatory signals. C) Storage niche. Quiescent stem cells are maintained in a niche until activated by external signals to divide and migrate (arrows) [51].

\subsection{Replicative senescence and immortality}

ESCS can divide indefinitely. However, when a cell becomes differentiated, it has a restricted proliferation capacity. MSCs also have a limited life span when grown in vitro. After 50-70 cell divisions [52], the cell cannot divide anymore, goes into replicative senescence (referred to as the "Hayflick phenomenon", in honour of Dr. Leonard Hayflick who was the first to publish it in 1965 [53]) and dies.

A phenomenon associated with senescence is telomere shortening. The ends of chromosomes are protected from degradation by a special chromatin structure known as the telomere. Telomeres consist of tandem repeats of TTAGGG, and during each cell division, repeats are lost as a result of 
incomplete replication. This successive shortening of telomeres eventually leads to loss of genetic material and results in cell death. ESCs express high levels of telomerase. The enzyme telomerase [54] adds telomeric repeats to the chromosome ends, thus protecting the shortening of the chromosomes. In most other cells, telomerase activity is low or undetectable. Telomerase has been proposed as the key to cellular immortality, turning off the clock, which counts off the number of cell division before senescence.

For research purposes, the unlimited availability of cells is highly desired. So besides ESCs, immortal or immortalized cell lines can be used. This can either be tumor cell lines, such as the HeLa cell line, which was isolated from a tumor biopsy of a patient called Henrietta Lacks (hence the name HeLa) in 1951, and has been in culture ever since. Alternatively, cells can be immortalized for example by introducing SV40 large TAg (Simian Vacuolating Virus 40 large T antigen), which is a powerful immortalizing gene. Cells that express SV40 large T antigen escape senescence but continue to lose telomeric repeats during their extended life span. After extended population doublings, they will eventually cease to proliferate as a result of chromosomal instability.

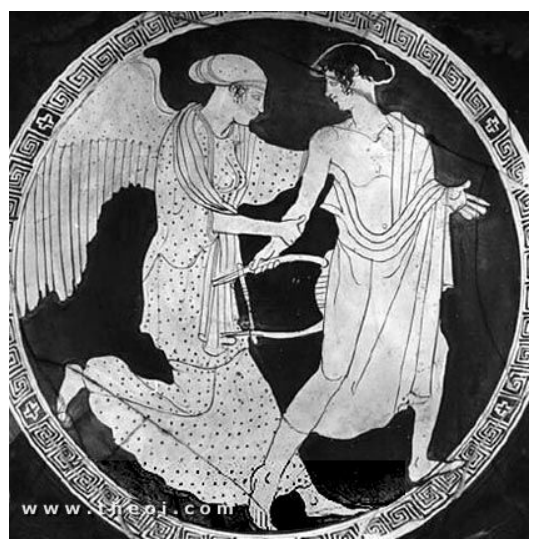

Figure 18. Immortality

Eos and Tithonos in happier times. The Greek goddess of the dawn, asked Zeus to make her very handsome lover Tithonos immortal, but forgot to ask for eternal youth. Tithonus indeed lived forever but grew more and more ancient. Museum Collection: British Museum, London, UK, taken from the website www.Theoi.com.

Cells can also be immortalized by restoring telomerase activity. When hTERT (human telomerase reverse transcriptase), the catalytic subunit of telomerase, is retrovirally introduced into human MSCs, the lifespan is these cells is extended. The cells maintain the ability to proliferate and differentiate over 3 years in culture. Thus, telomerization of human MSC by hTERT overexpression may maintain the stem cell phenotype [55]. 


\section{Classical Experiment: Multilineage potential of adult human mesenchymal stem cells}

The paper by Pittenger and co-workers in the Science issue of April 1999 [56] marked the broad introduction of the term "mesenchymal stem cells" for a population of cells originally identified by Friedenstein in the 1960s as plastic-adherent colony-forming units fibroblast (CFU-F) isolated from bone marrow [43]. Pittenger and co-workers obtained fifty bone marrow aspirates from 19-57 year old donors and selected the mononuclear fraction from the aspirate using a density gradient. Most of the cells from this fraction belong to the haematopoietic lineage and will not adhere when brought into culture. A small percentage of the cells (0.001-0.01\%) did adhere and they developed into symmetric colonies of cells. As such, the only difference with Friedenstein's isolation method is the purification of the mononuclear fraction. Flow cytometry analysis revealed that all cells were consistently positive for markers like, SH2, SH3, CD29, CD44, CD71, CD90 and CD106, but negative for the haematopoietic marker CD34. Despite this apparent homogeneity, MSCs isolated through this method still display large heterogeneity with respect to growth rate, phenotypic plasticity and colony morphology. Efficient expansion was achieved, with 50-375 million human MSCs within two cell passages. Human MSCs, however, do not express telomerase and are subject to proliferative senescence. The researchers further demonstrated efficient differentiation of human MSCs into the adipogenic, osteogenic and chondrogenic lineage by varying culture conditions (Figure 19).

This landmark paper, which has been cited 2500 times within 8 years after publication, strongly advocates human MSCs as a model system for questions of cell biological nature, such as cell fate decision, plasticity and senescence as well as a readily available source of cells for tissue engineering purposes. However, the name "mesenchymal stem cell" raised an ongoing debate on the nature of and nomenclature for mesenchymal cells with multi-potentiality isolated from various parts of the body including bone marrow and fat. The senescent phenotype of human MSCs in culture argues against their stemness. Therefore, many researchers rather use terms like marrow stromal cells, mesenchymal progenitor cells or skeletal progenitor cells to describe a population of cells, which cannot be discriminated from human MSCs on basis of marker gene expression or differentiation potential. 

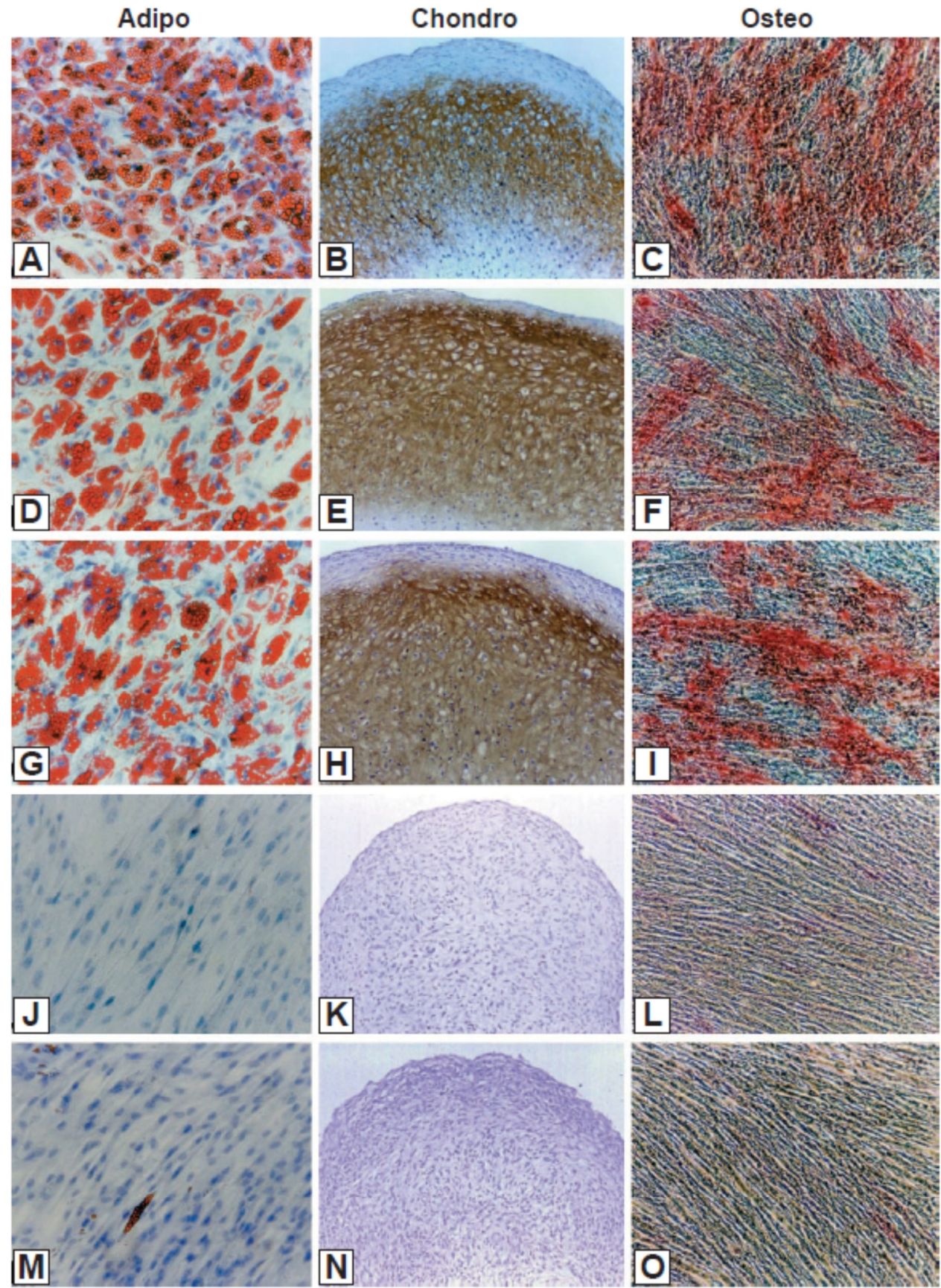

Figure 19. Isolated marrow-derived stem cells differentiate to mesenchymal lineages

Cultured cells from donors were tested for the ability to differentiate in vitro to multiple lineages. Donors (A through C) 158, (D through F) 177, and (G through I) 260 were each shown to differentiate appropriately to the adipogenic (Adipo), chondrogenic (Chondro), and osteogenic (Osteo) lineages. Adipogenesis was indicated by the accumulation of neutral lipid vacuoles that stain with oil red $O(A, D$, and $G)$, and such changes were not evident (J) with Hs27 newborn skin fibroblasts or (M) with 1087Sk adult mammary tissue fibroblasts. Chondrogenesis was 
shown by staining with the C4F6 monoclonal antibody to type II collagen and by morphological changes ( $B, E$, and $\mathrm{H}$ ), which were not seen by similarly culturing (K) Hs27 or (N) 1087Sk cells. Osteogenesis was indicated by the increase in alkaline phosphatase (C, F, and I) and calcium deposition, which was not seen in the (L) Hs27 or (O) 1087Sk cells [56].

\section{State of the Art Experiment: Reprogramming of adult cells into a pluripotent ESC-like state}

Human ESCs are a promising cell source for treatment of diseases and injuries, because they provide an unlimited supply of cell that can differentiate into cells of all three germ layers [12]. However, there are still technical (immune rejection of transplanted ESCs) and ethical problems (use of human embryos) before ESCs can be used in patients. The problem of rejection can be overcome by a technique called somatic cell nuclear transfer. In this technique, the nucleus from an egg is replaced by the nucleus of a somatic cell [57]. In another experiment, an ESC was fused with a somatic cell [58,59]. The ESC reprograms the somatic cell chromosomes to an embryonic state. Both experiments are technically challenging and do not solve the ethical problem. However, from these experiments it has become clear that somatic cells can be reprogrammed into an ESC-like state.

In a major breakthrough article, Takahasi et al. [60] hypothesized that the factors that play an important role in the maintenance of the ESC identity may be able to induce pluripotency in somatic cells. They selected 24 genes as candidate factors and after several experiments they narrowed this down to 4 transcription factors: Oct-4 and Sox2, which function in maintaining pluripotency in early embryos and ESCs, and c-Myc and Klf-4, which contribute to the maintenance ESC phenotype and their rapid proliferation. By retroviral introduction of the four transcription factors in mouse fibroblasts, these differentiated somatic cells were reprogrammed into a pluripotent state. These cells were called induced pluripotent stem (iPS) cells. The iPS cells displayed a similar morphology and growth phenotype as ESCs, expressed some ESC markers. After subcutaneous implantation into immuno-deficient mice, they formed teratomas containing tissues originating from of all three germ-layers. However, iPS cells were not identical to ESCs. The gene expression pattern and epigenetic state was different, and the iPS cells failed to produce chimaeras.

In three recent publications, a second generation of iPS cells was presented [17,61,62]. All groups used Nanog for the selection of reprogrammed cells, and this proved to be a better approach (Figure 20). The iPS cells obtained with this selection strategy did generate viable adult chimaeras (Figure 12), contributed to the germ line, and had an epigenetic state that was similar to that of ESCs.

If it is possible to reprogram human somatic cells as well, this technique may solve the ethical problems surrounding the use of human ESCs. Theoretically, it will enable the generation of patient- 
specific iPS cells, thereby circumventing immuno-rejection. However, there are still numerous problems to be solved. The efficiency of reprogramming should be increased. So far, the experiments were only performed with mouse cells. The method has to be applied to human cells, which most likely will require other factors. For therapeutic applications, the use of retroviruses to introduce the factors should be avoided.

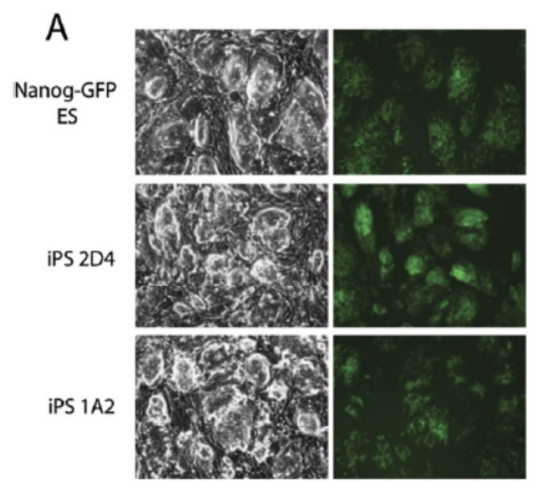

C

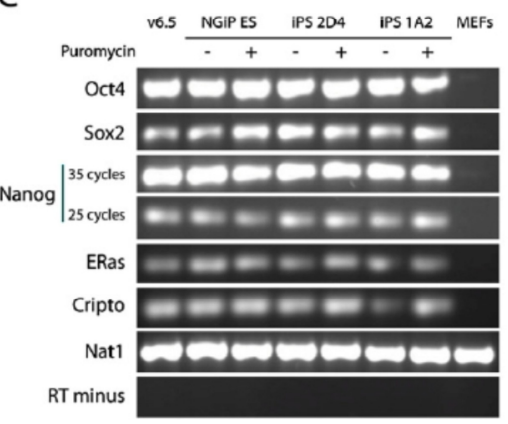

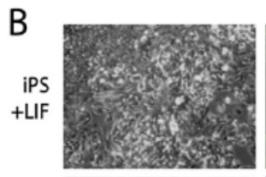

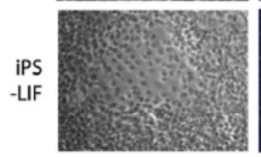

D
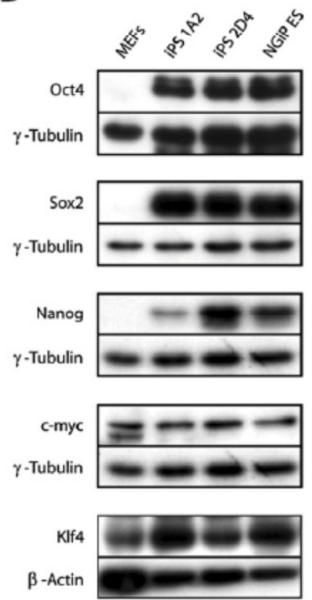
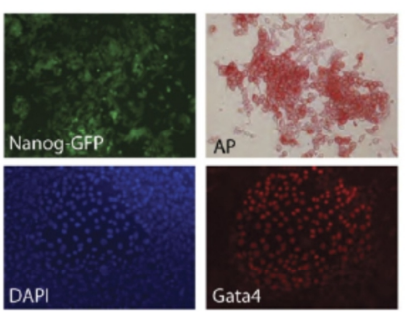

$E$
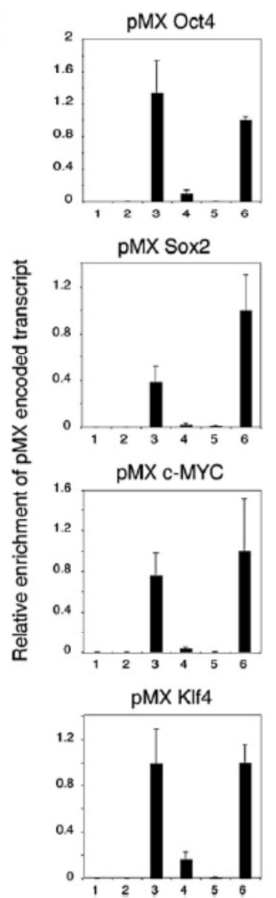

Figure 20. ESC-like Properties of Nanog-Selected iPS Cells

A) Morphology and Nanog promoter-driven GFP expression in ESCs (Nanog-GFP ES) and two iPS cell lines grown on feeders in the absence of puromycin selection. B) Effect of LIF withdrawal on iPS cells. Cells were grown for three passages without feeders. In the presence of LIF, 2D4 iPS cells maintain an ESC-like morphology, express endogenous Nanog as indicated by GFP expression, and are alkaline phosphatase (AP)-positive. Upon LIF withdrawal, iPS cells upregulate the primitive endoderm marker Gata4 as detected by immunostaining. A phase contrast image and counterstaining of the same cells with DAPI is shown. C) RT-PCR analysis of ESC marker gene expression in Nanog-GFP (NGiP) ESCs, and two iPS cell lines grown with and without continued puromycin selection, as well as in wild-type ESCs (V6.5) and MEFs as additional reference points. Primers for Oct4 and Sox2 are specific for transcripts from the respective endogenous locus. Nat1 was used as a loading control. D) Western blot analysis for expression of Nanog, Oct4, Sox2, c-myc, and KIf4 in iPS cell lines, MEFs, and Nanog-GFP (NGiP) 
ESCs. Anti-tubulin and anti-actin antibodies were used to control for loading. E) Quantitative PCR analysis of $\mathrm{pMX}$ retroviral transcription in (1) wild-type MEFs, (2) wild-type ESCs, (3) cells from the heterogeneous iPS line $1 \mathrm{~A} 2$ before sorting and subcloning, (4) 1D4 iPS, (5) 2D4 iPS, and (6) MEFs infected with the respective pMX virus. Transcript levels were normalized to $\beta$-actin. It should be noted that the retroviruses in the 2D4 iPS line appear completely silenced, whereas the heterogeneous $1 \mathrm{~A} 2$ line still shows abundant expression of the exogenous factors. Error bars represent the standard deviation of triplicate reactions. F) Teratoma derived from iPS line 1A2 showing differentiation into cell types from all three germ layers: epithelial structures (i), cartilage with surrounding muscle (ii), glandular structures (iii), and neural tissue (iv) [17].

\section{Future perspective}

Are stem cells a body self-repair kit? Can everybody get their own ESCs? Is it a hype, hope, or reality? Is it science, or science fiction? Is it too good to be true, too fast, too much, too soon, or too early to tell? The expectations are high, but can scientists meet them?

A lot of attention is being paid to stem cells, because their defining properties make them an ideal candidate to cure diseases. But a simple definition does not repair damaged tissue or replace failing organs. Much more knowledge is necessary, both fundamental and applied. If we look at the field of stem cell biology, the fundamental knowledge on the stem cell niche, self-renewal, early developmental processes, and epigenetics is growing every day. The next challenge is to apply this knowledge in the field of tissue engineering. Improved stem cell harvesting, stem cell culture, differentiation protocols and reprogramming of differentiated cells into a stem cell-like state will improve the chances of successful clinical applications. To achieve this, stem cell biologists and tissue engineers have to work together to bridge the gap between fundamental and applied science.

Human ESC research is still in its infancy: they were discovered just 10 years ago. As already mentioned in section 2.5.2 Application of human ESCS, ESC researchers in tissue engineering still face many technical hurdles. A recent international collaborative study from the International Stem Cell Initiative (ISCI) compared 59 human ESC lines from 17 laboratories from 11 countries [63]. The results from collaborative effort can be used to improve isolation, culture and differentiation protocols to make large-scale, clinical applicable culture available.

Adult stem cells have not been taken from the lab into the clinic at large scale. Bone marrow transplantation is by far the most successful therapy so far. In these transplants, a diseased of absent stem cell population is replaced by a fresh stem cell population. The body then instructs these cells to differentiate into the appropriate tissue. If we would like to replace damaged organs, we have to be able to differentiate the cells into a complex three dimensional tissue in vitro and/or in vivo. Therefore, we have to understand the complex cell-cell interactions that contribute to the function of the tissues. We might be able to differentiate a MSC into the osteogenic lineage, but that 
does not result in a piece of vascularized bone as seen in the body. Stem cells differentiated into cardiomyocytes will have to be coupled to the patient's heart cells to be functional. So it is not just (initiation of) differentiation that has to be investigated, in the end only a functional tissue will contribute to tissue repair. Many studies have been performed with mouse stem cells, or human stem cells implanted into an immuno-deficient mouse or rat. Because mice and men are not the same, successful results in mice will not automatically lead to (fast) successful results in humans.

The list of hurdles seems endless, but progress is being made. Progress towards the ultimate goal: an ESC-like cell for every patient (see section 5 State of the Art experiment)? It will take a lot of small steps, some breakthroughs, and unfortunately also unsuccessful experiments. During our efforts in the lab, Coldplay sings to us: "Nobody said it was easy". But realize that the combining factor between fundamental stem cell biology and engineered functional tissue is FUN! 


\section{CHAPTER 2 SKELETAL TISSUE ENGINEERING}

\section{USING EMBRYONIC STEM CELLS}

Jojanneke M. Jukes, Clemens A. van Blitterswijk, Jan de Boer

Institute for Biomedical Technology, Department of Tissue Regeneration, University of Twente, Enschede, The Netherlands

Don't you know

They're talkin' about a revolution

It sounds like a whisper

Talkin' about a revolution - Tracy Chapman - Tracy Chapman 


\title{
SKELETAL TISSUE ENGINEERING USING EMBRYONIC STEM CELLS
}

\begin{abstract}
Various cell types have been investigated as candidate cell source for cartilage and bone tissue engineering. In this review, we focused on chondrogenic and osteogenic differentiation of mouse and human embryonic stem cells (ESCS), and their potential in cartilage and bone tissue engineering. A decade ago, mouse ESCs were first used as a model to study cartilage and bone development and essential genes, factors and conditions for chondrogenesis and osteogenesis were unraveled. This knowledge, combined with data from differentiation of adult stem cells, led to successful chondrogenic and osteogenic differentiation of mouse ESCs, and later also human ESCs.

Next, researchers focused on the use of ESCs for skeletal tissue engineering. Cartilage and bone tissue was formed in vivo using ESCs. However, the amount, homogeneity and stability of the formed cartilage and bone were still insufficient for clinical application. The current protocols not only require improvement on differentiation efficiency, but also on ESC-specific hurdles such as the tumorigenicity and immunorejection. In addition, some of the general tissue engineering challenges such as cell seeding and nutrient limitation in larger constructs, will also apply to ESCs. In conclusion, there are still many challenges, but there is potential for ESCs in skeletal tissue engineering.
\end{abstract}




\section{Tissue engineering of cartilage and bone}

Tissue engineering aims at repairing or replacing damaged or diseased tissue. Cells, scaffold materials, or both form the basis of this approach. Tissue engineering is used for tissues that have a poor self healing capacity, such as cartilage, or when defects are too big for the body's capacity to heal itself, such as large bone defects.

For instance, in autologous chondrocyte transplantation (ACT) [64], articular cartilage is harvested from a minor load-bearing region of the knee. Chondrocytes are isolated and expanded in vitro and subsequently injected under a periosteal flap sutured over the defect. Since the first operation in 1987, patients are followed up continuously, and good results have been achieved, especially regarding the durability of the repair $[65,66]$. An alternative approach for the delivery and retention of the cells in the defect site is the use of a scaffold, which in addition can provide the mechanical support directly after surgery, thereby reducing the rehabilitation time. ACT and scaffold-based therapies for the repair of cartilage defects generally require millions of chondrogenic cells [67]. In order to obtain these cell amounts, in vitro cell expansion is required. It is well-known that chondrocytes dedifferentiate in monolayer culture [2]. Furthermore, in osteoarthritis, cartilage degenerates, due to a disturbed chondrocyte metabolism. As such, chondrocytes might not be the ideal cell source to repair the defect.

Current therapies for bone substitution include the use of autografs, allografts and synthetic materials. In the first strategy, often referred to as the golden standard, healthy bone is harvested from the patient and implanted into the bone defect. Good results have been obtained, but donor site morbidity and limited availability within the patient's body are drawbacks of the technique. More bone is available for allograft treatments, for which bone is harvested from a human cadaver. The bone has to be removed of all components which might induce an immune response, including cells. However, the risk of disease transmission cannot be ruled out, and allografts are less efficient than autografts. When autografts or allografts are not practicable, materials such as titanium alloys, for mechanical support, or ceramic materials or polymers to support bone growth have been used for hip replacement and other defects. However, poor integration with the surrounding bone due to mechanical and biological imcompatibility results in a need for bone graft substitutes. Cell-based bone tissue engineering might result in better bone healing and several cell types have been investigated. 


\subsection{Adult stem cells for tissue engineering}

The use of mature cell types such as chondrocytes and osteoblasts is associated with several drawbacks. The limited availability, donor site morbidity, dedifferentiation, and limited proliferative capacity urged researchers to study other cell types, in particular stem cells. The ability of stem cells to self-renew and differentiate into various cell types makes them an interesting source for tissue engineers. The best known example are bone marrow-derived stromal cells [56], also known as mesenchymal stem cells (MSCs), which can be differentiated into adipocytes, chondrocytes and osteoblasts. Other cells that have been differentiated into the chondrogenic or osteogenic lineage include MSCs isolated from adipose tissue derived after liposuction [68], fetal blood and liver [69], peripheral blood [70], umbilical cord [71], placenta [72], amniotic fluid [73,74] and human embryonic germ cells [75]. The low frequency and limited proliferative capacity are drawbacks of adult stem cells.

\subsection{Embryonic stem cells for tissue engineering}

Another potentially interesting cell type for tissue engineering is the embryonic stem cell (ESC). In 1981, mouse ESCs were isolated [10,11] (Figure 1A), followed by their human counterpart in 1998 [12] (Figure 1B).
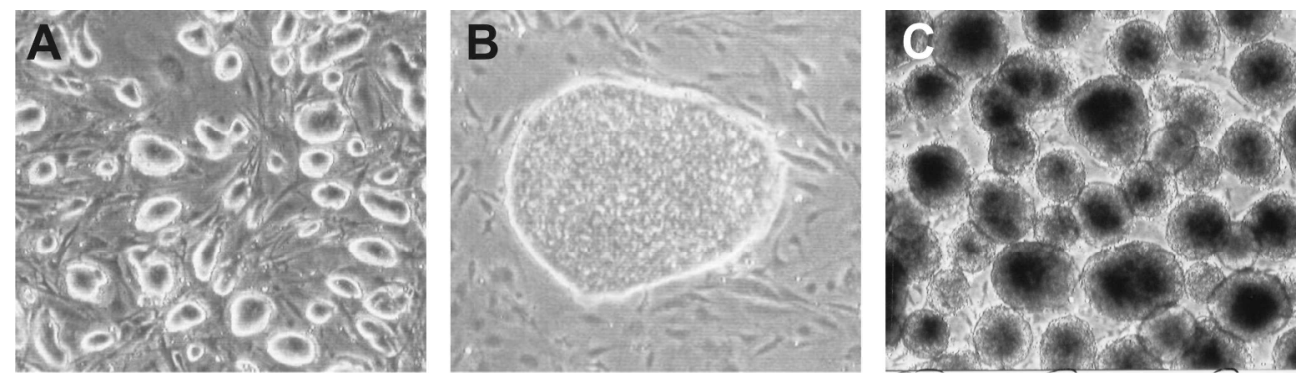

Figure 1. Embryonic stem cells and embryoid bodies

A) Mouse ESC colonies on a feeder layer of mouse embryonic fibroblasts. B) Human ESC colony on a feeder layer of mouse embryonic fibroblasts. C) Mouse EBs in suspension.

By definition, ESCs can self-renew and are pluripotent. Therefore, ESCs supply an infinite amount of cells which can be differentiated into all cell types of the human body, in contrast to adult stem cells which are at best multipotent and generate limited amount of cells in vitro. Stem cell biologists have developed protocols to proliferate the ESCs indefinitely while remaining undifferentiated. Equally challenging is the development of defined protocols to differentiate ESCs into the desired cell type in vitro and in vivo. The first step in most ESC differentiation protocols is the formation of embryoid bodies (EBs) (Figure 1C). EBs are spontaneously forming free-floating aggregates of ESCs, in which cells differentiate at random into lineages of all three germ layers: endoderm, mesoderm, and 
ectoderm. These intact EBs, or single cells derived from these aggregates, so called EB cells, are used in the subsequent directed differentiation steps.

The ability of ESCs to form cartilage or bone is well-known, as these tissues are readily observed in teratomas. A teratoma, a benign tumor in which tissue from all three germ layers can be recognized, develops when undifferentiated ESCS are injected into various organs of an animal. However, the directed and controlled chondrogenic and osteogenic differentiation of ESCs had to be determined. Strategies for the differentiation of MSCs and ESCs into the chondrogenic and osteogenic lineage have been reviewed in 2004, but data on ESC was limited at that time [76,77]. The strategies include culturing in defined media to which chondro- or osteoinductive cytokines, growth factors and chemical factors have been added, the use of instructive substrata such as ECM components, coculturing and the addition of conditioned media, the application of physical stimuli, and genetic manipulation. Many of these protocols are based on protocols established for adult stem cells, chondro- and osteoprogenitors, or mature chondrocytes and osteoblasts.

ESCs have the potential to become all cell and tissue types of the body, and during EB formation, cells differentiate at random. During embryonic development, chondrogenesis and osteogenesis are closely intertwined. Therefore, it should not be surprising that many of the chondrogenic supplements are also involved in osteogenic differentiation. In fact, many of the cytokines, growth factors and signaling pathways described for chondro- and osteogenesis play a role in the development of many tissues. A well-defined differentiation protocol is required to direct all ECSs into the chondrogenic or osteogenic lineage.

A decade ago, researchers started to differentiate ESCs into the chondrogenic and osteogenic lineage. The aim of many papers was to study chondrogenesis and osteogenesis, and not specifically the use of ESCs for tissue engineering or regenerative medicine applications. Using ESCs, the early steps of chondrogenesis and osteogenesis can be studied, for which adult stem cells and chondrocytes or osteoblasts are too mature. Another advantage is the relative ease to stably transfect ESCs and to obtain high cell numbers of transfected cells. By generating Sox $9^{+/}$and Sox $9^{-1-}$ ESC lines, Sox9 was identified as the first transcription factor that is essential for chondrocyte differentiation and cartilage formation [78]. Gene knockout or overexpression helped in elucidating the influence of transcription factors and genes on chondrogenesis and osteogenesis [79-83]. By creating chimaeric mice, the function of these genes can be studied in the developing embryo or living animals. In other studies, mouse ESCs were used as a screening system to identify factors that are both necessary and sufficient for cartilage and bone formation $[84,85]$. The lessons learned from 
ESCs as a model to study cartilage and bone development, can result in improved protocols for more successful application of ESCs in cartilage and bone tissue engineering.

First studies were performed using mouse ESCs, and differentiation approaches were translated to human ESCs. After establishing the chondrogenic and osteogenic potential of ESCs, tissue engineers focused on the formation of cartilage and bone in combination with scaffolds. In this review, we further comment on the current challenges that have to be solved before ESCS can be used in the clinic, such as optimization of culture protocols, tumorigenicity of ESCS and immune rejection of allogeneic ESCs.

\section{Chondrogenic differentiation of ESCs}

To determine whether ESCs have indeed differentiated into the chondrogenic lineage, markers that characterize cartilage were investigated. Articular cartilage is composed of chondrocytes, the extracellular matrix (ECM) they secrete, and water. The major components of the cartilage ECM are collagen type II and the proteoglycan aggrecan. Two chondrocyte-specific transcription factors have been identified, Sox9 [78] and scleraxis [86]. Cartilage has some distinct histological features: round cells in a lacuna surrounded by large areas of ECM (Figure 2A).
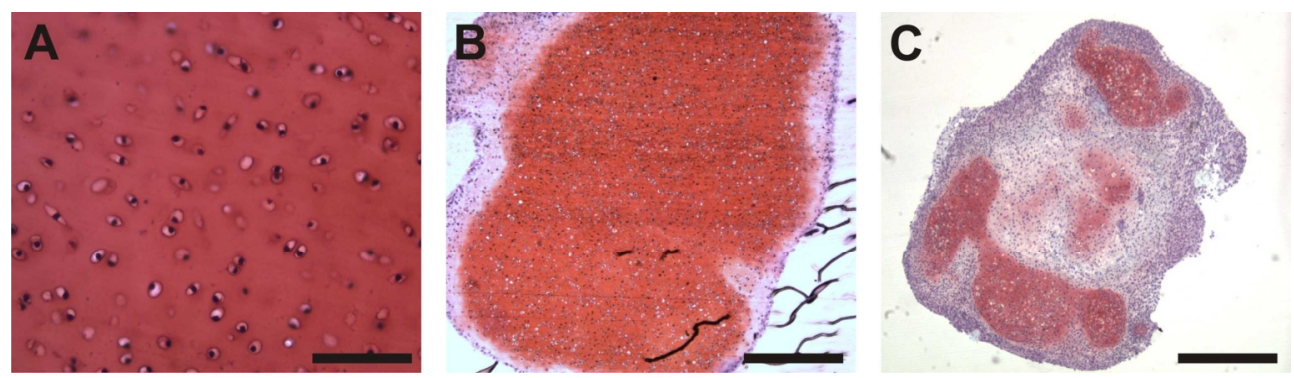

\section{Figure 2. Cartilage}

A) A cross section of bovine articular cartilage, in which the chondrocytes can be recognized in their lacunae, surrounded by ECM. Glycosaminoglycans are stained pink by Safranin-O. Scale bar represents $100 \mu \mathrm{m}$. B) After 21 days in pellet culture, bovine chondrocytes, which were harvested from the cartilage in figure $2 \mathrm{~A}$, secreted cartilage ECM. Scale bar represents $500 \mu \mathrm{m}$. C) After 21 days in pellet culture in serum-free chondrogenic medium, mouse ESCs have formed cartilage nodules. Scale bar represents $500 \mu \mathrm{m}$.

\subsection{Spontaneous chondrogenic differentiation of mouse ESCS}

In the first studies, intact mouse EBs were cultured on tissue culture plastic in basic ESC proliferation medium and in the outgrowth of the attached EBs, cartilaginous regions were observed [87]. Various stages of chondrogenesis, such as condensed mesenchymal cells, chondroprogenitor cells, mature and hypertrophic chondrocytes could be recognized by analyzing the expression pattern of mesenchymal and cartilage-related transcription factors and genes $[87,88]$ and electron-microscopic 
analysis [89]. Thus, mouse ESCs can differentiate into the chondrogenic lineage not only in teratomas, but also in vitro, though with varying efficiency between several mouse ESC lines [90].

\subsection{Chondrogenic differentiation of ESCs in a co-culture system}

The formation of tissues in the developing embryo is heavily influenced by factors that are secreted by, or through direct cell-cell interactions with, the surrounding cells. This can be mimicked in vitro in a co-culture system, in which ESCs are differentiated into the chondrogenic lineage in the direct or indirect presence of chondroprogenitor cells or mature chondrocytes.

Antibiotic-resistant mouse ESCs were combined with progenitor cells derived from the limb buds of the developing embryo in micromass culture. After 4 days, antibiotic treatment allowed only the ESCs to survive. These cells continued to differentiate, and cartilaginous nodules were formed. Direct cell-cell contact was important in this system, as no chondrogenic differentiation was observed when conditioned medium from limb bud progenitor cells was added to mouse ESCs [91]. In an indirect co-culture system, human ESCs were separated from primary human nasal chondrocytes [35] or bovine chondrocytes [92] by a well insert. Factors that were secreted by the mature chondrocytes were able to diffuse through the membrane and stimulate the chondrogenic differentiation of the human ESCs.

\subsection{Growth factor-induced chondrogenic differentiation}

Some of the chondrogenic factors that are secreted by the surrounding cells have been identified. Rather than adding the more mature cells, the isolated factors can be added to the ESC cultures. In the following experiments, the basic proliferation medium was supplemented with growth factors, such as members of the transforming growth factor beta (TGFß) super family, to direct and enhance chondrogenic differentiation. TGFß1 alone did not enhance, but actually slightly reduced the amount of cartilage nodules derived from mouse ESCS [87]. The addition of TGFß3 and parathyroid hormone under low serum conditions after retinoic acid treatment of EBs favored chondrogenic differentiation [93].

When bone morphogenetic protein (BMP)-2 or -4 was added early in the differentiation protocol when EBs were in suspension, chondrogenesis was induced [87]. The chondrogenic effect of BMPs was time-dependent. Rather than chondrogenic differentiation, BMP4 induced ESCS into the osteogenic lineage when added to retinoic acid-treated EBs during the entire differentiation procedure (discussed below) [93]. The addition of BMP2 and TGFß1 during EB formation, followed by the addition of insulin, ascorbic acid (AA) and BMP2 during the differentiation period resulted in the most profound stimulation of cartilage-specific gene expression [94]. Thus, promotion of the 
chondrogenic lineage was dependent on the combination of the growth factors, hormones and other supplements, their concentration and time of application.

\subsection{Effect of cell morphology on chondrogenic differentiation}

Cartilage tissue can easily be recognized by the round shaped chondrocytes embedded in ECM. When chondrocytes are isolated from cartilage and grown in monolayer culture, the cell morphology changes from round to fibroblast-like and the cytoskeleton reorganizes from a cortical distribution towards bundled stress fibers of actin filaments. As a consequence, chondrocytes dedifferentiate and switch from collagen type II to collagen type I expression. Dedifferentiated chondrocytes can redifferentiate by culturing them in pellets or in a hydrogel in which cell-cell or cell-hydrogel contact is present. Cell morphology can also be influenced by culturing cells in the presence of a cytoskeleton-disrupting agent such as cytochalasin, which has previously been used to induce chondrogenesis of mesenchymal limb bud cells [95]. When ESCs were treated with cytochalasin, cell morphology changed from fibroblast-like towards round, the stress fibers were disrupted and actin filaments reorganized. Cytochalasin-treated EB-derived cells expressed more Sox9 and collagen type II after 4 hours. After 5 days, the treated cells stained positive for the collagen type II protein, but the expression of the collagen type II gene returned to the level of control cultures [96]. Thus, cytochalasin treatment and the resulting change in cell morphology had an effect on chondrogenesis of ESCs. However, we should realize that a transient rise in gene expression does not necessarily mean that significantly more ECM will be secreted by the cells. Therefore, additional experiments will have to be performed to see the long term effect of changing cell morphology on cartilage formation.

\subsection{Effect of aggregation on chondrogenesis}

In the studies described above, the ESCs were cultured on tissue culture plastic. This 2D environment does not seem to be the most logical choice to form a 3D tissue. One of the earliest steps in chondrogenesis is the formation of a high cell density aggregate, which allows cell-cell interactions between the mesenchymal cells and with the surrounding cells. Whereas cartilage tissue does not consist of a single cell layer, but is a 3D structure, the flat EB plating culture results in flat cells and does not allow extensive cell-cell interactions. Mesenchymal or precartilage condensation can be mimicked in a micromass or pellet culture system (Figure 2B), in which the cells are cultured in a high cell density aggregate. Cartilage formation of mesenchymal cell lines and MSCs was enhanced in pellets [97]. The influence of aggregation on chondrogenic differentiation of mouse ESCs was further investigated. Chondrogenic differentiation of mouse ESCs on tissue culture plastic 
was mainly observed in regions where cells clustered into 3D-like chondrogenic nodules. Rather than waiting for the cells to form the aggregates themselves, micromass culture or pellets of EB cells were formed in basic proliferation medium, and compared with plated EBs. Chondrogenesis was indeed enhanced in the 3D cultures [98]. In addition, pellets allow analysis of the cartilage morphology. By making cross sections of the pellets, round cells could be recognized in ECM. Thus, aggregation enhanced chondrogenesis of ESCs and created a 3D tissue, which is a feature of cartilage.

\subsection{Cartilage formation in serum-free chondrogenic medium}

Instead of culturing in ESC basic proliferation medium supplemented with cartilage-specific growth factors, ESCs can also be differentiated in medium used to drive adult stem cells or primary cells into the chondrogenic lineage. Chondrogenic differentiation of rabbit and human MSCs can be achieved in serum-free medium containing proline, AA, sodium pyruvate, insulin-transferrin-selenious acid (ITS+), dexamethasone (Dex) and TGFß1 or TGFß3 $[97,99,100]$. Cartilage nodules were formed in pellets of mouse ESC-derived EB cells cultured in this serum-free chondrogenic medium containing TGFß3 [101,102] (Figure 2C). We even observed cartilage formation in the absence of TGFß3 [102], but under both conditions the formed tissue was not homogeneous and other cell types were also present in the pellets.

Chondrogenic differentiation was observed in regions of human EB outgrowth when human ESCS were cultured in serum-free chondrogenic medium containing BMP2. Micromass cultures in the presence of BMP2 again showed the beneficial effect of high cell density on cartilage formation [103]. When human EBs were cultured for 4 weeks in serum-free chondrogenic medium with a cocktail of growth factors, some ECM was produced. These EBs or the dissociated EB cells were cultured for another 4 weeks. EB cells formed pellets and produced more ECM, whereas EBs formed loose aggregates [104]. When these self-assembled constructs were cultured under hypoxia, thereby mimicking the conditions of native cartilage, more collagen type II was produced, and the human ESCs displayed better biomechanical functionality [105].

\subsection{Chondrogenic differentiation of human ESC-derived MSCS}

Rather than starting the differentiation protocols with undifferentiated human ESCs, or cells derived from EBs, some studies have first generated a cell population similar to MSCs. To initiate mesenchymal differentiation, human ESCs were cultured on a feeder layer of murine OP9 stromal cells, which have the potential to induce hematopoietic differentiation of mouse ESCs. After 40 days, cells were sorted by Fluorescence Activated Cell Sorting (FACS) for CD73, a marker expressed in 
adult MSCs. The CD73-positive fraction expressed a set of surface markers considered to define MSCs, and were negative for hematopoietic markers [106]. Functional characterization was performed by differentiating the cells into mesenchymal tissues such as fat, cartilage and bone.

During embryonic development, mesenchymal cells do not only arise from mesoderm, but also from the neural crest. After differentiation of human ESCs into neural rosettes, a CD73-positive fraction could be isolated, which could be differentiated into the adipogenic, chondrogenic and osteogenic lineage [107]. Thus human ESCs appear to be able to differentiate into mesenchymal-like cells, from both mesoderm [106] and neural crest [107]. However, both protocols might be rather complicated and lengthy, with low efficiency for tissue engineering applications.

In a simplified protocol, spontaneously differentiated cells were separated from the undifferentiated human ESC colony by mechanical dissociation. The scraped cells were cultured in basic medium, and expressed similar CD markers as MSCs [108]. Multipotency was determined by differentiating the cells into the osteogenic and adipogenic, but not chondrogenic, lineage. There are differences in morphology and differentiation capacity between various batches. By using a similar approach, mesenchymal-like cells were derived by passaging the EB outgrowth several times. The cell population became more homogeneous, and morphologically similar to MSCs [109]. The human EBderived mesenchymal-like cells expressed some MSC surface markers, but not CD73, the marker used in the above-mentioned studies for selection of the mesenchymal fraction [106,107]. Although the derivation of ESC-derived mesenchymal-like cells was simplified, the chondrogenic differentiation capacity has to be optimized.

From the above-described studies, one can conclude that it is possible to direct both mouse and human ECS into the chondrogenic lineage, and form cartilage tissue. The positive effect on cartilage formation of some growth factors and supplements and of 3D culture over 2D plating has been confirmed. However, no protocol resulted in the formation of large amounts of homogeneous tissue. Besides cartilage nodules, many other tissue types are also formed. Some protocols are quite complicated and will not be easily transferred into a clinical setting. In conclusion, the efficiency and homogeneity of cartilage formation should be further improved.

\section{Cartilage tissue engineering using ESCs}

Even though only some requirements for cartilage formation in 2D and small 3D systems had been defined, researchers already started culturing and differentiating the ESCs in a tissue engineering set up. To be able to repair larger defects in clinical practice, the formation of larger constructs is necessary. Generally, this involves the use of a scaffold material. Requirements for successful tissue 
engineering application include high cell numbers, homogeneous and high cell density seeding, and good nutrient flow throughout the scaffolds for sustained viability and homogeneous differentiation. The first requirement is often a problem for primary and adult stem cells, but not for ESCs because they can proliferate indefinitely. However, the last prerequisites pose an equal challenge for ESCs.

\subsection{Cartilage tissue engineering using polymeric scaffolds}

Mouse ESCs that were stimulated into the chondrogenic lineage in basic medium supplemented with $A A$, insulin and TGFß were seeded onto polycaprolactone scaffolds. The cells attached to the scaffold, and after subcutaneous implantation in mice some Alcian blue-positive cells were observed [110]. However, these cells did not form tissue with typical cartilage morphology. Co-cultured human ESCs were seeded onto poly-D, L-lactide scaffolds and implanted subcutaneously in the back of immuno-deficient mice. After 5 weeks, ECM was produced on the scaffolds, and collagen type II was detected by immunostaining [35].

When mouse EB cells or intact EBs were seeded onto a poly(ethylene oxide terephthalate)poly(butylene terephthalate) scaffold and differentiated in serum-free medium containing TGFß3, cartilage formation was observed. However, the seeding was inhomogeneous and the efficiency was rather low [102]. For homogeneous seeding, the scaffold's pores have to be fully interconnected to allow cells to enter the center of the scaffold. On the other hand, cells should not wash out by medium flow, or sink to the bottom of the scaffold due to gravitational forces. Optimized scaffold architecture and dynamic seeding and culturing might improve seeding efficiency and homogeneity. However, the seeding of ESCs in chondrogenic medium is complicated by the absence of serum proteins, which resulted in easy detachment of the cells from the polymeric fibers [102]. Serum proteins enhanced cell attachment on scaffolds, but they inhibited the chondrogenic differentiation of mouse ESCs [102] (Chapter 5). A careful balance between medium flow and cell attachment has to be maintained, to successfully seed and differentiate ESCs on scaffolds.

An alternative to direct seeding is using a gel to seed cells on a scaffold. Human EB cells were combined with Matrigel and seeded onto a thin poly(lactic-co-glycolic acid)/poly(L-lactic acid) scaffold. The human ESC proliferation medium was supplemented with TGFß1 to direct the cells into the chondrogenic lineage. Cartilaginous tissue was indeed formed in vitro [36].

\subsection{Cartilage tissue engineering using hydrogels}

Hydrogels cannot only be used as a seeding method, but also as a scaffold. A homogeneous high cell density can be achieved in hydrogels. Gels like agarose and alginate have been used to study cartilage formation by chondrocytes. Mouse EB cells were encapsulated in various hydrogels and the 
seeding was indeed homogeneous, but most cells died within 1-7 days [102], whereas bovine chondrocytes survived the entire 21 day culture period. In Matrigel, only a superficial layer of EB cells survived (Figure $3 \mathrm{~A}$ ) and differentiated into the chondrogenic lineage (Figure 3B). This implies that cell-matrix and cell-cell interactions improve viability of the EB cells in a hydrogel. Indeed, when intact EBs instead of EB cells were seeded in the hydrogels, the majority of the EBs remained viable during the entire 21 day culture period [102].
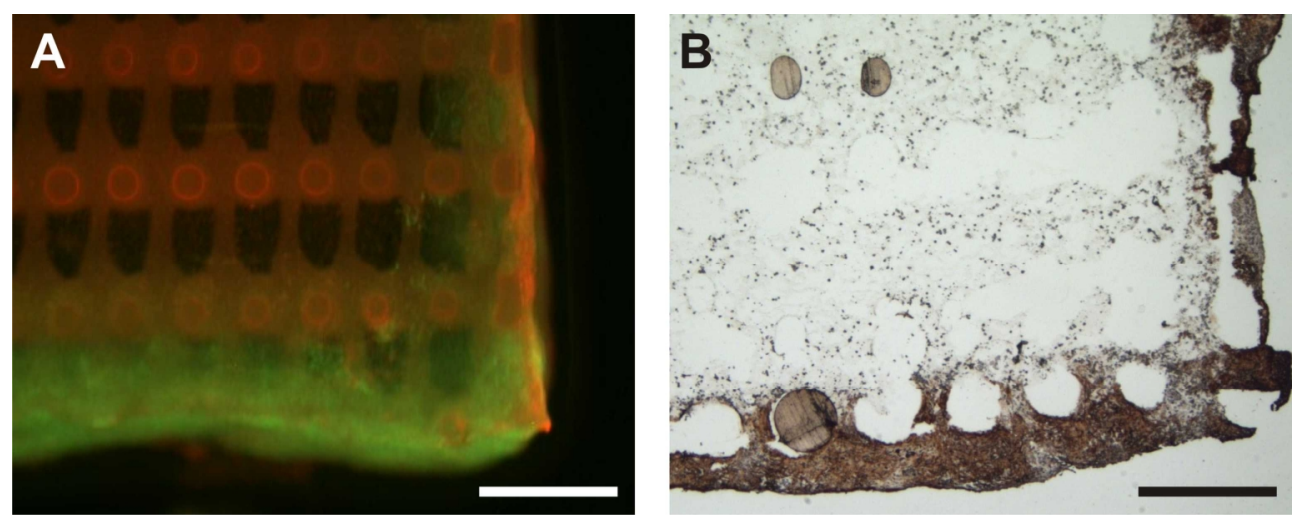

Figure 3. Mouse ESCs in a hydrogel

A) Mouse ESC-derived EB cells were mixed in Matrigel and then injected into a poly(ethylene oxide terephthalate)-poly(butylene terephthalate) scaffold. After 21 days, a superficial layer of viable cells is present, whereas cells in the center of the constructs died. Viable cells are indicated by green staining and dead cells by red staining. The scaffold fibers display red background staining. Scale bar represents $100 \mu \mathrm{m}$. B) Cartilage formation by mouse ESCs in the viable tissue layer as seen in figure 3A. Immunostaining for collagen type II is visualized by brown staining. Scale bar represents $100 \mu \mathrm{m}$.

Next, the chondrogenic differentiation of EBs in gel systems was investigated. When intact mouse EBs were encapsulated in alginate disks, and cultured either in basic proliferation medium, or medium supplemented with Dex, chondrogenesis was not enhanced, when compared with plated EBs [98]. However, when mouse EBs were encapsulated in a poly(ethylene glycol)-based hydrogel and cultured in serum-free chondrogenic medium containing TGFß1, a significant increase in cartilage markers was observed compared to 2D plating [111]. Addition of glucosamine to the chondrogenic medium resulted in improved cartilage matrix production [112]. Cartilage formation by EBs was also observed when cells were seeded in either Matrigel or agarose [102]. When EBderived cartilage in either Matrigel or agarose was implanted in immuno-deficient mice for 3 weeks, some cartilage was observed in vivo. However, the implanted cartilage was not very stable. Upon degradation of the gel, most of the cartilage was lost. In addition, teratoma formation was observed in these experiments, even though samples had been pre-cultured in vitro [102]. 
Human EB-derived mesenchymal-like cells were seeded in functionalized poly(ethylene glycol) (PEG)-based hydrogels and cultured in chondrogenic medium containing TGFß1. The addition of collagen type I or hyaluronic acid did not result in chondrogenic differentiation, but the addition of RGD-peptides induced the formation of a cartilaginous matrix by human ESC-derived mesenchymallike cells [109]. It is known that mechanical compression can enhance chondrogenic differentiation [113]. When human EB-derived mesenchymal-like cells were encapsulated in a hydrogel and 1 cycle of mechanical stimulation was applied the expression of cartilage genes was increased [114]. Whether mechanical stimulation of ESCs result in more cartilage formation and what the optimal loading regime would be, has to be determined.

Co-cultured human ESCs were also seeded in PEG-RGD gels, and cartilage-like tissue was formed and cartilage-specific genes were expressed [92] at higher levels than in PEG hydrogels. When these cellgel constructs were implanted, cartilage tissue was observed after 12 and 24 weeks in vivo.

In conclusion, mouse and human ESCs have been differentiated into the chondrogenic lineage and cartilage was not just formed on plates and in pellets, but also in gels and on scaffolds. Some typical tissue engineering difficulties were encountered such as limited nutrient diffusion, and homogeneous seeding. In vitro, regions of cartilage tissue were observed on the scaffolds, but still heterogeneous as also seen in pellets. Few in vivo studies were performed, and it is clear that these protocols require major improvement. The regions of cartilaginous tissue were small, and in some studies, typical characteristics of cartilage were not observed. The efficiency and stability of in vitro and in vivo cartilage formation has to be improved to obtain clinically relevant amounts of cartilage for tissue engineering applications.

\section{Osteogenic differentiation of ESCs}

Bone (Figure 4) is mainly composed of cells, inorganic and organic matrix and water. Three mesodermal-derived cell types constitute bone. Osteoblasts secrete uncalcified matrix called osteoid, the osteocytes reside in little lacunae surrounded by calcified matrix and bone lining cells are in a resting state. The osteoclasts, which originate from hematopoietic precursors, degrade matrix. Their differentiation from ESCs [115-124] will not be included in this manuscript. Unlike cartilage, bone is a vascularized tissue. Inorganic bone matrix consists of carbonated calciumphosphate apatite. Collagen type $I$ is the main component of the organic matrix and proteoglycans and non-collagenous proteins such as osteopontin, osteonectin, osteocalcin and bone sialoprotein (BSP) form the remaining fraction. Osteoblasts express alkaline phosphatase (ALP), an enzyme which 
plays a role in calcification. Two bone-specific transcription factors, osterix [125] and runt-related transcription factor $2 /$ core binding factor alpha 1 (runx2/cbfa1) [126], have been identified.
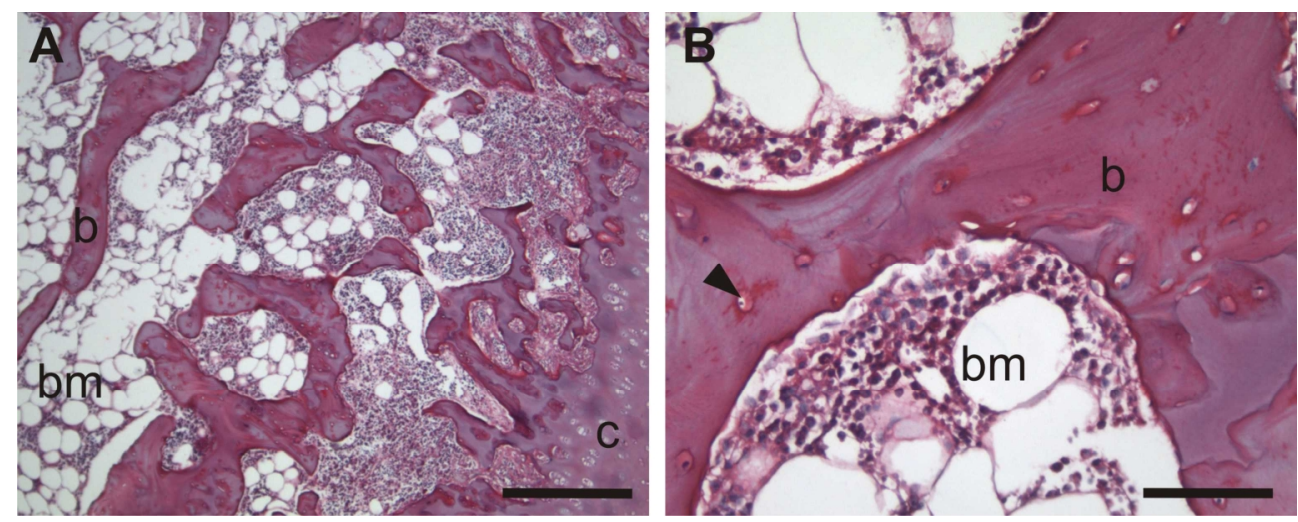

\section{Figure 4. Bone}

A) Haematoxylin and eosin stained cross section of bone derived from a bovine knee. The articular cartilage (c) of the femoral head can be seen on the right and the underlying bone (b) and bone marrow (bm) in the marrow cavities on the left. Scale bar represents $500 \mu \mathrm{m}$. B) At higher magnification, osteocytes (black arrowhead) can be recognized in their lacunae in the bone matrix (b). Scale bar represents $100 \mu \mathrm{m}$.

Osteogenic differentiation in vitro can be analyzed by looking at mineral deposition, ALP activity and osteogenic gene expression. Bone tissue can form in vivo, which can be defined histologically by identifying bone lining cells and osteocytes and by looking at the organized structure of lamellar bone. In addition, bone marrow can be found.

\subsection{Growth factor-induced osteogenic differentiation of ESCS}

Some spontaneous osteogenic differentiation of ESCs was observed in basic medium [127]. By using similar approaches as described above for chondrogenic differentiation, the osteogenic fraction could be increased. Growth factors and cytokines widely used to promote osteogenic differentiation of primary osteoblasts, pre-osteoblasts and adult stem cells [128] are AA, ß-glycerophosphate (BGP) and Dex. Supplementing ESC medium with AA and BGP [127] or the trio of supplements [129-132] significantly increased the amount of bone nodules and the expression of osteogenic markers in mouse and human ESC cultures. Osteogenic differentiation was also observed when intact EBs, rather than dissociated EB cells, were cultured in the presence of osteogenic supplements $[133,134]$. Thus, osteogenic protocols could be transferred from adult (stem) cells to ESCs. Researchers also translated a protocol for adipogenic differentiation of mouse ESCS [135] to osteogenic differentiation [136] and later to chondrogenic differentiation [93]. In this protocol, EBs were treated with retinoic acid (RA) from day 3-5. When RA-treated EBs were cultured in mineralization medium, osteogenic markers were expressed, but only few mineralized areas were formed [136]. 
Addition of BMP4 [93], BMP2 [136], or compactin [136], a member of the statin family shown to increase bone density and osteoblast number in vivo [137], greatly enhanced the amount of bone nodules.

Not only biological supplements can induce osteogenic differentiation. Inorganic stimuli derived from bioactive glass could induce the growth and differentiation of primary osteoblasts [138]. The soluble ions released from bioactive glass were as effective as Dex at inducing the formation of mineralized nodules, and the addition of bioactive glass extract to Dex-treated cultures resulted in an increased amount of bone nodules [139]. This suggests that scaffolds of inorganic materials used in bone tissue engineering, can enhance osteogenic differentiation.

\subsection{Timing of osteogenic supplementation}

In previous studies, it was recognized that the effect of many supplements is time-dependent. Late administration of Dex resulted in an increase of bone nodules for both mouse $[127,140]$ and human ESCs [141]. The addition of the calcium-regulating hormone vitamin D3 instead of Dex also increased the number of calcified cells [142], but only when the osteogenic supplements were added after the EB formation step.

In a microarray study, control ESCs were compared with vitamin D3-treated cells and a change in expression of genes related to pluripotency and osteogenic differentiation was observed [143]. RA was expressed early and BMP2 was expressed at later stages of differentiation. This is in agreement with the observations that RA only enhanced osteogenic differentiation when added during the EB formation phase [136], and not when added later during differentiation and mineralization [127]. Adding osteogenic supplements at the wrong time even resulted in a decrease in osteogenesis, as seen when BMP2 was added during early stages of differentiation [143]. Early administration of BMP4 resulted in chondrogenic differentiation [87], whereas continuous stimulation resulted in osteogenesis [93].

Thus, by timing the supplementation of RA, BMP2, Dex and vitamin D3 the differentiation of ESCs into the osteogenic lineage could be significantly increased.

\subsection{Osteogenic differentiation of ESCs in a co-culture system}

Direct and indirect co-culture systems were also investigated for osteogenic differentiation of ESCs. Indirect co-culture of mouse ESCS with primary calvarial osteoblasts isolated from fetal mice resulted in five times more bone nodules, compared to control ESCs in the absence of osteoblasts [127]. The cell-cell interactions and BMPs secreted by primary bone-derived cells, stimulated human ESCs into the osteogenic lineage in a direct co-culture system [144]. In an alternative co-culture 
system, the use of bone was avoided. Cell extracts derived from human ESC-derived osteogenic cultures induced undifferentiated human ESCs into the osteogenic lineage [145].

Rather than inducing cells into the osteogenic lineage, another co-culture aimed at increasing the mesodermal fraction before and during EB formation, by culturing mouse ESCs in the presence of medium conditioned by the human hepatocarcinoma cell line HepG2. The most efficient osteogenic differentiation was observed when EBs formed for 1 and 3 days. Longer EB culture times resulted in the spontaneous differentiation towards beating cardiomyocytes and reduced osteogenic differentiation [146]. Thus, careful timing of the EB formation time of conditioned medium-treated mouse ESCs enhanced osteogenic differentiation.

\subsection{Influence of the EB step on osteogenic differentiation}

It is unclear whether the EB formation step, a not well-characterized step during which the cells differentiate at random, is essential or if cells might be directly differentiated into the desired lineage.

HepG2-treated and untreated mouse ESCS still formed bone nodules [147] or cartilage [148] when the EB step was completely omitted, or shortened as described above [146]. However, these studies did not compare osteogenic or chondrogenic differentiation without and with EB formation, and therefore one cannot conclude which protocol is more efficient. When the EB formation step was omitted, calcium deposition by human ESCs was slightly delayed [130]. In contrast, another study concluded that human ESCs formed bone nodules earlier and at higher efficiency without the EB step [149], as also seen for mouse ESCs [150]. The formation of free-floating EBs was compared with initiation of differentiation in monolayer [151]. All four human ESC lines could be differentiated into the mesodermal and osteogenic lineage, although with varying efficiency. In fact, 3 lines exhibited greater osteogenic potential after monolayer culture, whereas one cell line expressed more bonespecific markers after EB formation [151].

From the studies described above we can conclude that EB formation is not essential, but we cannot conclude whether EB formation step is beneficial for osteogenic differentiation.

Even though most researchers based their protocols for osteogenic differentiation of ESCs on protocols established for human MSC, parameters such as timing of osteogenic supplements and their concentration varied between the experiments. For example, not all groups used Dex, and when added to the osteogenic medium, the concentration varied from $10^{-4}$ [138] to $10^{-8} \mathrm{M}$ $[132,149,151]$, which might have a major influence on osteogenic differentiation. The ESC-specific step of EB formation was different on many points. In some experiments, EB formation was omitted, 
and when EBs were formed, the duration and the supplements added varied. Later during differentiation, either intact EBs, or EB cells were used. Even though the most optimal protocol has not been established yet, mineralization and osteogenic gene expression were observed, although at varying levels.

\section{Bone tissue engineering using ESCs}

To apply ESCs in the field of bone tissue engineering, the protocols will have to be transferred from tissue culture plastic to scaffolds. The formation of bone tissue by ESCs can be analyzed in in vivo experiments.

\subsection{Osteogenic differentiation of ESCs on scaffolds}

In the first study describing 3D osteogenic differentiation of mouse ESCS, RA-treated EBs were first cultured for 3 weeks in 2D on tissue culture plastic in mineralization medium. Next, these differentiated cells were trypsinized and seeded onto polylactic acid scaffolds. Cells attached, grew, and after another 4 weeks in mineralization medium, they secreted ECM which stained positive for mineralization by von Kossa staining, and they expressed osteocalcin and osteopontin. These patterns were similar to the control 2D plates [134].

To investigate whether the 3D environment is enhancing osteogenic differentiation, as described above for chondrogenic differentiation of ESCS, EB cells were encapsulated in a peptide hydrogel, Puramatrix, and cultured in mineralization medium [152]. Mineralization was observed both in 2D and 3D. Slightly higher levels of osteopontin and collagen type I were observed in the 3D hydrogel cultures compared to 2D tissue culture plastic. ALP activity on the other hand, was higher in nonosteogenic control than in osteogenic cultures. However, ALP is also expressed by undifferentiated cells, and the number of Oct4-positive cells was higher in this 3D system [152]. This indicates that the 3D system enhances osteogenic differentiation, but also the undifferentiated state of the cells. All in all, the 3D environment seems to mimic the cell's natural environment, being bone or the stem cell niche, better than $2 \mathrm{D}$ culture plastic.

\subsection{Osteogenic differentiation of ESCs in vivo}

Both mouse and human ESCs could be successfully stimulated into the osteogenic lineage in vitro. However, for tissue engineering applications, it is essential that the cells can contribute to bone formation in vivo. Despite the many in vitro studies, no studies have been published about in vivo bone formation by mouse ESCs. Our group has observed that stimulation of the cells with compactin, BMP2 or Dex resulted in mineralization in vitro, as described above. However, when 
mouse ESCs were seeded on ceramic scaffold particles, cultured in the various osteogenic media for 7 or 21 days and subsequently implanted in subcutaneous pockets of immuno-deficient mice, no bone was formed [129]. Thus, in vitro osteogenic differentiation of mouse ESCs did not lead to bone formation in vivo (manuscript submitted).

Human ESC-derived EB cells were cultured in the presence of osteogenic supplements AA and BGP for 14 days, and Dex was added to this medium for another 24 hours. These stimulated cells were then seeded onto poly-D,L-lactide scaffolds and implanted subcutaneously onto the back of immuno-deficient mice for 5 weeks. Discrete areas of mineralization were observed, and osteocalcin was expressed by the implanted cells [141]. However, the formation of bone in which osteocytes and bone lining cells could be recognized was not observed.

Implantation in the peritoneal cavity of osteogenic and control human ESC-derived EB cells in injection chambers for 11 weeks resulted in the formation of mineralized areas [153]. However, from the presented data, we conclude that control samples were equally positive as osteogenic cultures. Apparently, osteogenic supplementation for 4 days before implantation was not long enough to enhance osteogenic differentiation of human ESCs to levels higher than spontaneous osteogenic differentiation. Similar conclusions could be drawn from the in vitro data [130].

Human ESCs which were co-cultured in vitro for 14 days on a feeder layer of PBDs as described above [144], were seeded onto porous composite scaffolds using fibrinogen and implanted in immuno-deficient mice [154]. After 4 and 8 weeks in vivo, small patches of bone-like tissue were observed between the fibrous connective tissue. More mineralized tissue was observed on ESCseeded than on empty scaffolds. The addition of BMP2 in the fibrinogen-cell mixture further enhanced osteogenic differentiation.

Thus, some indications of bone-like and mineralized tissue formation by human ESCs in vivo were present, but the tissue formed did not resemble bone histologically.

\section{Endochondral bone tissue engineering using ESCs}

Bone can be formed by two different mechanisms in the embryo. In the first, neural crest-derived mesenchymal cells condense and differentiate into osteoblasts. This direct approach has been used in studies that attempted to differentiate ESCs into bone. In the other mechanism, paraxial and lateral plate mesoderm-derived mesenchymal cells aggregate and form cartilage which matures and calcifies. Blood vessels invade the calcified cartilage, which serves as a template for bone deposition by osteoblasts. As the direct differentiation into the osteogenic lineage did not result in bone 
formation, the endochondral approach was investigated. The first step in endochondral ossification is the maturation of the cartilage template, which becomes hypertrophic, as indicated by the switch from the expression of collagen type II, a cartilage-specific marker, to collagen type $\mathrm{X}$, expressed by hypertrophic chondrocytes.

\subsection{Full chondrogenic maturation of ESCs in vitro}

Indications of hypertrophic maturation and calcification of ES-derived chondrocytes were observed during in vitro chondrogenic differentiation. During spontaneous differentiation of attached mouse EBs, the expression of cartilage markers reduced, which coincided with the appearance of collagen type X, a marker for hypertrophic cartilage, and bone markers $[88,155,156]$. Expression of osteogenic markers could also be stimulated by switching from chondrogenic medium to hypertrophic medium [101] or by adding osteogenic supplements such as vitamin D3, BGP [94] or BMP2 [103]. Thus, in the same culture, several steps of chondrogenic and osteogenic differentiation could be recognized.

\subsection{Endochondral bone formation in vivo}

The initial steps of endochondral ossification can be studied in vitro. ESCs are able to produce fully matured chondrocytes and subsequently osteogenesis is observed. However, the actual formation of bone via this indirect approach has to be studied in vivo. Therefore, mouse ESCs were seeded on scaffolds, and differentiated into the chondrogenic lineage for 21 days in serum-free chondrogenic medium containing TGFß3. At this time, cartilage tissue was observed and collagen type II was expressed. When these cartilage tissue-engineered constructs were implanted subcutaneously in immuno-deficient mice, the cartilage became hypertrophic, calcified, became surrounded by bone tissue and was ultimately replaced by bone within 21 days (Figure 5). The newly formed bone was aligned with osteoblasts, and osteocytes were visible in the mature and mineralized bone tissue.

Chondrogenic induction for 3 or 7 days was not sufficient, as bone formation was only observed after induction for 14 and 21 days which resulted in the formation of a cartilage template. Endochondral bone formation was not just observed in subcutaneous pockets. When ESC-derived cartilage was implanted on a ceramic disc in a critical size cranial defect in an immuno-deficient rat, bone was formed as well. Using the endochondral approach, for the first time directed and reproducible in vivo bone formation using ESCs was demonstrated in ectopic and orthotopic sites. The process is very robust. Bone was observed in all experiments where an ESC-derived cartilage template was formed in vitro [129]. However, when we followed the same approach for human 
ESCs, the efficiency of cartilage formation was too low to observe endochondral bone formation using human ESCs. Thus, endochondral ossification might be a new approach for bone tissue engineering using ESCs.

\section{0 days}

14 days

21 days
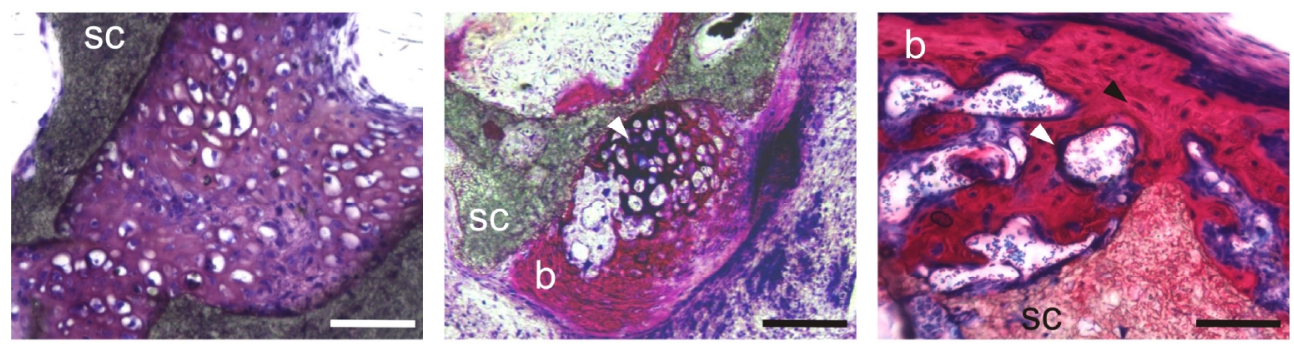

Cartilage

\section{Bone}

Figure 5. Endochondral bone formation by mouse ESCs

Mouse ESCs were seeded on ceramic scaffolds (sc) and differentiated into the chondrogenic lineage for 21 days. Subsequently, these samples were implanted subcutaneously in nude mice ( 0 days in vivo). After 14 days in vivo, hypertrophic cartilage (white arrowhead) can be recognized, surrounded by newly formed bone tissue (b). After 21 days in vivo, bone tissue with osteocytes (black arrowhead) and bone lining cells (white arrowhead) can be recognized. Thus in time, the cartilage matured, served as a template for bone formation and was replaced by bone tissue. Cartilage tissue was stained purplish pink by thionin ( 0 days), bone tissue was stained reddish pink by basic fuchsin (14 and 21 days). Scale bar represents $100 \mu \mathrm{m}$.

\section{Current and future challenges}

Some of the basic requirements for cartilage and bone tissue engineering using ESCs have been established. The protocols have been improved on many points, but currently, both cartilage and bone formation in vivo is not efficient enough for clinical application. Besides enhancing chondrogenic and osteogenic differentiation, some ESC-specific challenges still have to be solved. The first steps to solve some of the challenges have already been addressed and will be described below.

\subsection{Upscaling of culture and differentiation of ESCS}

The current protocols for ESC maintenance and differentiation are rather time-consuming and labor intensive, especially protocols for human ESCs. The propagation of human ESCs often involves mechanical dissection by micropipettes, a process which requires concentration, time and 
experience. Feeder-free culture of human ESCS on normal culture plastics, and passaging using trypsin would make up-scaling more realistic. In many protocols, growth factors and other cytokines are used. For large scale culture, efficient use of these expensive supplements will have to be implemented.

To simplify the differentiation of ESCs, osteogenic differentiation without the EB formation step has been described above [149-151]. A bioreactor system in which maintenance of ESCs, EB formation and differentiation can be combined by simply changing the medium has been described for osteogenic differentiation [157]. Mouse ESCs were encapsulated in alginate/gelatin beads, and cultured in a rotating $50 \mathrm{ml}$ bioreactor vessel in ESC maintenance medium. When medium was changed to EB medium for a period of 5 days, the proliferating cells formed aggregates, which expressed the mesodermal marker flk-1. Beads were cultured for another 21 days in osteogenic medium and mineralization was observed. ESCs can thus be differentiated in this simplified system, which is amenable for scale-up. ESCs could also be seeded and proliferated on gelatin-based microcarriers in a spinner flask. When proliferation medium was changed to osteogenic medium, indications of osteogenic differentiation were observed [158].

\subsection{Animal product-free culture of ESCS}

Most isolation, proliferation and differentiation protocols use animal-derived products, which can be contaminated with pathogens. Much attention was drawn to the risk of pathogen transfer to humans when prion diseases such as Creutzfeldt Jakob, also known as mad cow disease, were discovered. It was also discovered that human ESCS can take up and express non-human sialic acid Neu5Gc from either feeder cells or culture media [37], which might provoke an immune-response upon transplantation. Therefore, ideally, protocols will be free of animal product before ESCs can be applied in human clinical therapy. This means that ESCs can no longer be isolated and maintained using mouse embryonic fibroblasts as feeder cells, medium cannot contain animal serum proteins, and animal-derived supplements such as trypsin and growth factors. Several studies have optimized the maintenance protocols by using human feeder cells, or ECM as a substitute for feeders, and serum-replacement instead of bovine serum. However, most protocols still contain animal products in one of the many steps that are required from isolation to application of ESCs. Full replacement of animal-derived products might result in an undesired high cost of culture and differentiation, which makes the use of ESCs less attractive for clinical application. 


\subsection{Patient-specific ESCs}

As ESCS do not exist in the human body, they cannot be isolated from patients. Therefore, immunological rejection upon implantation of scaffolds seeded with allogeneic ESCs can be expected. One approach to circumvent this problem is to match ESCs to the patient, similar to organ transplantations. Since ESCs have an indefinite proliferation capacity, large banks of ESCs can be established, which can be screened to find a potential human leukocyte antigen (HLA) match [159]. Patients will likely have to take immunosuppressive drugs for the rest of their lives. More radical approaches would be the genetic alteration of ESCs to match the patient, or the very technically and ethically challenging therapeutic cloning.

Recently, patient-specific ESC-like cells have been created. Somatic cells have been reprogrammed to a pluripotent state and are referred to as induced pluripotent stem cells (iPS cells). Transcription factors that are involved in the maintenance of pluripotency, such as Oct3/4 and Sox2, and genes involved in the maintenance and rapid proliferation of ESCs, c-Myc and Klf4, were introduced into mouse fibroblasts by retroviral transfection [60]. These differentiated fibroblasts then reprogrammed into an embryonic-like state, as indicated by their morphology and expression of ESC markers. By improving the protocol, three groups then reported the generation of viable adult chimeras, which indicated that the iPS cells contributed to the formation of all cell and tissue types in the mouse $[17,61,62]$.

Subsequently, human cells have been reprogrammed to pluripotency by the introduction of the 4 above-mentioned factors in adult human dermal fibroblasts [160], or a combination of Oct4, Sox2, Nanog and Lin28 [161]. These human iPS cells expressed ESC markers and could form EBs containing cells of the three germ layers. They could be differentiated into neural and cardiac cells, and after implantation teratomas formed in which many tissues, amongst others cartilage, were observed. The transgenicity of iPS cells might be a drawback for clinical applications, but new protocols using for example RNAi or small molecules could avoid viral transduction. Further research will have to be performed to investigate the chondrogenic and osteogenic differentiation of iPS cells for skeletal tissue engineering applications.

\subsection{Tumorigenicity of ESCS}

The ability to form benign tumors in vivo, so called teratomas, is a well-known characteristic of ESCs. Undifferentiated mouse ESCs were injected into mouse knee joints or into a subcutaneous pocket and analyzed after 1 to 8 weeks. In total, 22 out of 25 mice developed subcutaneous tumors, whereas 8 out of 25 knees contained a tumor. The knee tumors were smaller than the subcutaneous tumors, but large enough to destroy the knee joint after 8 weeks. Cartilage nodules were observed 
both in subcutaneous and knee joint tumors [162]. When undifferentiated mouse ESCs were implanted into osteochondral defects created in the patellar groove of immunosuppressed rats, no tumors were observed up to 8 weeks. Cartilaginous tissue was observed, which indicated the chondro-inductive effect of the osteochondral defect on ESCs [163]. Next, the influence of mechanical stimulation on cartilage and tumor formation by mouse ESCs was investigated. Rats with mouse ESCs in osteochondral defects in the patellar groove were elevated of their hind limbs by tail suspension. In one group, free motion of the knee joint was allowed, whereas in the second group the knee joints were immobilized, due to which the patella did not fully cover the defect. Tumors were formed in all the immobilized knee joints, whereas fibrocartilage was amongst the regenerated tissue when knee movement was allowed [164]. The confined space outside the defect created by the movement of the patella, might prevent tumor growth, as also suggested when subcutaneous and knee joint tumors in mice were compared [162]. It appears that the environment of the osteochondral defect site has a beneficial effect on osteochondral differentiation and a negative effect on tumor formation by undifferentiated mouse ESCs.

In the experiments described above, undifferentiated ESCS were used, which are known to have tumorigenic potential. Therefore, most researchers differentiate the cells in vitro into the desired lineage, before they are used in animal studies. However, we observed that 3 weeks of in vitro differentiation of mouse ESCs in chondrogenic medium, which resulted in the presence of fully differentiated cartilage tissue, was not sufficient to eliminate the formation of teratomas in vivo [102]. Even though the population of undifferentiated cells is greatly reduced upon differentiation, every remaining uncommitted cell can grow uncontrolled when implanted into a defect site. Strategies have to be developed to be absolutely sure that no remaining undifferentiated cells are implanted into the patient, as this can have a detrimental effect. Besides undifferentiated cells, cells that are committed to another lineage are also undesired. For example, cardiomyocytes, which form rather spontaneously during differentiation of ESCs, do not contribute to the repair of a cartilage or bone defect.

\subsection{Homogeneous tissue formation by ESCS}

The optimization of differentiation protocols in order to obtain homogeneous tissue can focus on improving the differentiation efficiency or at purifying the heterogeneous cell population. This can either be negative selection by which undifferentiated cells are separated from the rest of the population, or positive selection for chondrogenic or osteogenic markers.

A combination of both optimization and positive selection resulted in more homogeneous cartilage formation by ESCs. To stimulate the formation of mesodermal cells during EB formation, BMP4 was 
added at this stage. The mesodermal fraction was sorted from the EB cells by selecting for lateral plate mesoderm marker vascular endothelial growth factor receptor 2 (VEGFR-2 or flk-1) and paraxial mesoderm marker platelet-derived growth factor receptor alpha (PDGFR $\alpha$ ) [101]. These mesodermal progenitor cells, expressing either flk-1, PDGFR $\alpha$, or both, were capable of forming cartilage nodules in a pellet culture, when cultured in serum-free medium containing TGFß3. The negative fraction did not form cartilaginous tissue. The PDGFR $\alpha$-positive fraction was also able to form cartilage nodules in the absence of TGFß3 [101], as also observed for unsorted cells [102]. By adding PDGF-BB or BMP4 to the differentiation medium, larger pellets filled with cartilage matrix could be obtained. Thus, the combination of growth factors can stimulate a mesodermal fraction of mouse ESCs towards more homogeneous cartilage formation.

Similarly, osteogenic ESCs were sorted by magnetically activated cell sorting (MACS) using Cadherin11, a cell adhesion molecule expressed at high levels during the early stages of osteoblasts differentiation. When the cadherin-11-positive population was cultured for another 2 weeks in mineralization medium, almost homogeneous bone nodule formation was observed, whereas the cadherin-11-negative fraction did not show osteogenic differentiation [140].

The choice of selection marker is critical, but difficult. By choosing an early marker, the cells might still have the potency to differentiate towards undesired lineages. By choosing a late marker, the cells might already be embedded in their ECM and therefore less accessible for markers and for selection techniques which mostly require a single cell solution.

\section{Conclusion}

Differentiation of ESCs into the chondrogenic and osteogenic lineage caught the attention of scientist from the beginning of this century. More recently, researchers started to investigate the potential of ESCs in cartilage and bone tissue engineering. The earlier studies merely analyzed chondrogenic and osteogenic markers. However, we observed that increased expression of the collage type II gene does not necessarily mean that cartilage tissue will be formed, and mineralization in vitro is not a good indicator for in vivo bone formation. Some analyses reveal no information on the distribution of cartilage and bone in the sample, nor tell something about the heterogeneity, both of which are important parameters.

The search for the optimal protocol continues, and as a result, it is often difficult to compare the results of the articles. The subtle or large differences between differentiation protocols might have a bigger influence on chondrogenesis or osteogenesis then currently known. In addition, there will be variation between the various mouse and human ESC lines used in various studies. Even though 
there are similarities between mouse and human ESCs, there are even more differences. Not all mouse data can simply be extrapolated to human ESCs. Last but not least, the amount of in vivo data is still limited. The formation of sufficient amounts of functional and stable cartilage and bone requires further research.

Besides increasing the efficiency of cartilage and bone formation, ESC-specific challenges will also have to be addressed. These current disadvantages affect researchers investigating the use of ESCS in any clinical application, and hopefully a combined effort can be made to find the solutions to make the use of ESCs safe.

Progress has been made in the last years, but cartilage and bone tissue engineering using ESCs is still in its infancy. Luckily, this is the period when cartilage and bone develop rapidly in the human body, thus we can be optimistic about the future perspectives.

\section{Acknowledgements}

The authors gratefully acknowledge the support of the Dutch Technology Foundation STW grant TPG 5923, the Dutch Program for Tissue Engineering and the TeRM Smart Mix Program of the Netherlands Ministry of Economic Affairs and the Netherlands Ministry of Education, Culture and Science.

The authors declare that they have no conflict of interest. 



\section{CHAPTER 3 A NEWLY DEVELOPED \\ CHEMICALLY CROSSLINKED DEX-PEG HYDROGEL FOR CARTILAGE TISSUE ENGINEERING}

Jojanneke M. Jukes ${ }^{1}$, Leonardus J. van $\operatorname{der} \mathrm{Aa}^{2}$, Christine Hiemstra ${ }^{2}$, Theun van Veen ${ }^{1}$, Pieter J. Dijkstra ${ }^{2,3}$, Zhiyuan Zhong ${ }^{3}$, Jan Feijen ${ }^{2}$, Clemens A. van Blitterswijk ${ }^{1}$, Jan de Boer ${ }^{1}$

${ }^{1}$ Institute for Biomedical Technology, Department of Tissue Regeneration, University of Twente, Enschede, The Netherlands

${ }^{2}$ Institute for Biomedical Technology, Department of Polymer Chemistry and Biomaterials, University of Twente, Enschede, The Netherlands

${ }^{3}$ Biomedical Polymers Laboratory and Jiangsu Key Laboratory of Organic Chemistry, College of Chemistry, Chemical Engineering and Materials Science, Soochow University, Suzhou 215123, PR China

The swell season

Glenn Hansard and Markéta Irglová - The Swell Season 


\title{
A NEWLY DEVELOPED CHEMICALLY CROSSLINKED DEX- PEG HYDROGEL FOR CARTILAGE TISSUE ENGINEERING
}

\begin{abstract}
Cartilage tissue engineering, in which chondrogenic cells are combined with a scaffold, is a cellbased approach to regenerate damaged cartilage. Various scaffold materials have been investigated, amongst which hydrogels. Previously, we have developed dextran-based hydrogels which form under physiological conditions via a Michael type addition reaction. Hydrogels can be formed in situ by mixing a thiol-functionalized dextran (Dex-SH) with a tetra-acrylated star poly(ethylene-glycol) (PEG-4-Acr) solution. In this manuscript we describe how the degradation time of Dex-PEG hydrogels can be varied from 3 to 7 weeks by changing the degree of substitution of thiol groups on dextran. The degradation times increased slightly after encapsulation of chondrocytes in the gels. The effect of the gelation reaction on cell viability and cartilage formation in the hydrogels was investigated. Chondrocytes or embryonic stem cells (ESCS) were mixed in the aqueous dextran solution and we confirmed that the cells survived gelation. After a 3-week culturing period, chondrocytes and ESCderived embryoid bodies were still viable and both cell types produced cartilaginous tissue. Our data demonstrate the potential of dextran hydrogels for cartilage tissue engineering strategies.
\end{abstract}




\section{Introduction}

Hydrogels are hydrated networks of crosslinked hydrophilic polymers. Hydrogels are applied in day to day life as contact lenses and the absorbent material in disposable diapers. These applications reflect some of the interesting characteristics of hydrogels: biocompatibility and high water content, which make hydrogels suitable for biomedical applications such as wound dressings, drug delivery systems and tissue engineering. Due to their high water content, many hydrogels are compatible with proteins and cells. By controlling the permeability and degradation profile of the hydrogel, encapsulated drugs or proteins can be released locally in a controlled fashion. The mechanical properties of hydrogels parallel those of soft tissues, which make hydrogels suitable candidates for tissue engineering of adipose tissue and blood vessels. Hydrogels are also investigated for cartilage tissue engineering, as the encapsulation of cells provides the desired 3D environment, and the extracellular matrix of cartilage is also highly hydrated. The design of hydrogels can be directed towards a controlled degradation allowing it to be replaced by newly formed tissue. Cells can be combined with the hydrogel precursors before gelation and functional groups or growth factors can be incorporated into the hydrogel to enhance tissue formation. In situ forming hydrogels are highly interesting, as the cell-laden hydrogels can be injected into the defect site by a minimally invasive surgical procedure.

A wide variety of chemically and physically crosslinked hydrogels, based on natural materials and synthetic polymers, have been developed and studied in recent years. Natural materials include the proteins fibrin, collagen and gelatin, and the polysaccharides hyaluronic acid, alginate, dextran and chitosan. Examples of synthetic polymers are block-copolymers of poly(ethylene glycol) (PEG) and poly(lactic acid) (PLA), poly(glycolic acid) (PGA), poly(glycolic acid-co-lactic acid) (PGLA) or poly( $\varepsilon$ caprolactone) (PCL).

The aqueous polymer solution can be turned into a gel by physical or chemical crosslinks. The noncovalent bonds of physically crosslinked hydrogels, for example stereocomplexed hydrogels [165], result in mechanically weak hydrogels. The physical interactions are reversible, resulting in disruption of the gel upon a change in, for example, temperature or $\mathrm{pH}$. An advantage is that these gels can generally be formed under mild conditions. Chemical crosslinking results in more stable hydrogels due to the covalent bonds formed. The properties of chemically crosslinked hydrogels can be varied by the amount of crosslinks introduced and the hydrophilic-hydrophobic ratio. Further customization can be achieved by varying the concentration of polymer and the polymer length. 
Reactive crosslinkers or initiators, or crosslinking conditions can be toxic for included cells or may lead to modification of biological compounds. Examples of chemical crosslinking methods include photopolymerization of acrylated polymers by UV or visible light and redox polymerization. Recently, more mild conditions for chemical crosslinking have been developed, such as the Michael reaction [166-169]. The addition of a nucleophile to an $\alpha, ß$-unsaturated carbonyl compound, a type of conjugate addition, results in the formation of covalent bonds.

We are interested in applying hydrogels for cartilage repair. Due to the poor self healing capacity of cartilage, surgical intervention is generally required. Techniques such as mosaicplasty, microfracturing or subchondral bone drilling generally result in low quality cartilage. A cell-based therapy aimed at regenerating the damaged cartilage is tissue engineering, in which chondrogenic cells are generally combined with a scaffold material and applied in the defect site. In autologous chondrocyte implantation ( $\mathrm{ACl}$ ), culture expanded chondrocytes are injected in the defect site behind a periosteal flap [64]. Alternatively, chondrocytes can be combined with a scaffold material, such as a hydrogel, to achieve high cell density, homogeneous seeding, retainment of the cells in the defect, and incorporating chondrogenic compounds. Our approach is to mix chondrogenic cells in the hydrogel prior to gelation, and allow formation of cartilage extracellular matrix before the hydrogel completely degrades. Chemically crosslinked hydrogels that can be formed under mild conditions meet our requirements. Recently, we developed dextran hydrogels prepared by Michael type addition [170,171].

Dextran is a bacterial-derived polysaccharide, which is commercially available. Dextran particles have been widely used as chromatography matrices (Sephadex) and as cell carriers (Cytodex). Dextran has been used as a blood plasma expander and dextran hydrogels have been investigated for drug delivery applications [172]. Dextran-based hydrogels are highly hydrophilic and biocompatible and the degradation products can be excreted through the kidneys as long as the molecular weight of the original dextran components is below the filtration threshold of the kidney. The hydroxyl groups of dextran allow the introduction of functional groups by conjugation $[173,174]$. When dextran is functionalized with reactive groups such as thiols, chemically crosslinked hydrogels can be formed via Michael type addition between thiols and polymers containing acrylate or vinyl sulfone groups. The reaction is catalyzed by a weak base, which is present under physiological conditions. The precursor solutions can be injected at the defect site and the hydrogel will form in situ. The crosslinking reaction is self-selective: the polymer components react with each other, and side reactions with proteins are minimal [166]. 
We developed a range of dextran hydrogels with various characteristics. Dextran was functionalized with either thiol (SH) or vinyl sulfone (VS) groups. Thiol-functionalized dextran (Dex-SH) could form a hydrogel with tetra-acrylated star PEG (PEG-4-Acr) or vinyl sulfone-functionalized dextran (Dex-VS) [170]. Dex-VS reacted with multifunctional mercapto PEG (PEG-2-SH or PEG-4-SH) or dithioerythritol (DTE) [171]. Hydrogels of Dex-VS crosslinked with PEG-4-SH have been investigated for controlled release of proteins [175]. Based on gelation and degradation time, we decided to investigate hydrogels of Dex-SH crosslinked with PEG-4-Acr (Dex-PEG) for cartilage tissue engineering using both chondrocytes and stem cells, and compared the results with agarose gels.

\section{Materials and methods}

\section{Bovine chondrocyte culture}

Articular cartilage was harvested from the femoral patellar groove of freshly slaughtered calves and dissected into $1-\mathrm{mm}$ pieces. Chondrocytes were isolated via overnight digestion in $0.15 \%$ type II collagenase (Worthington Biochemical, Lakewood, NJ). Fresh chondrocytes were used at passage 0 and cultured in chondrocyte medium, which was composed of Dulbecco's Modified Eagle's Medium (DMEM) high glucose (Invitrogen, Carlsbad, CA)), 10 mM HEPES (Invitrogen), 10\% fetal bovine serum (FBS, Cambrex, Walkersville, MD), $100 \mathrm{U} / \mathrm{ml}$ penicillin (Invitrogen), $100 \mu \mathrm{g} / \mathrm{ml}$ streptomycin (Invitrogen), $0.2 \mathrm{mM}$ ascorbic acid (ASAP, Sigma, St. Louis, MO), $0.1 \mathrm{mM}$ non-essential amino acids (NEAA, Sigma) and $0.4 \mathrm{mM}$ proline (Sigma).

\section{Mouse ESC culture}

Mouse ESC line iB10 was plated at a density of 5,000-10,000 cells $/ \mathrm{cm}^{2}$ on gelatin-coated tissue culture flasks. Mouse ESCs were cultured in 50\% mouse ESC proliferation medium consisting of DMEM (Biowhittaker, Basel, Switzerland) containing $4.5 \mathrm{mg} / \mathrm{ml} \mathrm{D-glucose,} \mathrm{10 \%} \mathrm{FBS} \mathrm{(selected} \mathrm{batch}$ for mouse ESC culture, Greiner, Kremsmünster, Austria), $100 \mathrm{U} / \mathrm{ml}$ penicillin, $100 \mu \mathrm{g} / \mathrm{ml}$ streptomycin, $4 \mathrm{mM}$ L-glutamine (Invitrogen), $0.1 \mathrm{mM} \mathrm{NEAA}$, and 50\% of Buffalo rat liver cellconditioned mouse ESC proliferation medium [176]. Prior to use, $1000 \mathrm{U} / \mathrm{ml}$ Leukemia Inhibitory Factor (Esgro, Chemicon International, Billerica, MA) and $50 \mu \mathrm{M}$ 2-mercaptoethanol (Invitrogen) were added to the medium. Cells were grown at $37^{\circ} \mathrm{C}$ in a humidified $5 \% \mathrm{CO}_{2}$ incubator and passaged with $0.05 \%$ trypsin/EDTA (Invitrogen) before reaching confluence.

A common first step in differentiation protocols of ESCs is the formation of embryoid bodies (EBs). EBs were formed by culturing mouse ESCs at 20,000 cells $/ \mathrm{cm}^{2}$ in non tissue culture-treated 6-well plates (Greiner) in $4 \mathrm{ml}$ EB medium consisting of DMEM, 10\% FBS, $100 \mathrm{U} / \mathrm{ml}$ penicillin, $100 \mu \mathrm{g} / \mathrm{ml}$ 
streptomycin, $4 \mathrm{mM}$ L-glutamine, $0.1 \mathrm{mM}$ NEAA and $50 \mu \mathrm{M}$ 2-mercaptoethanol for 4 days. To obtain a single cell suspension, EBs were dissociated with $0.25 \%$ trypsin/EDTA. ESC-derived EB cells were used for differentiation experiments.

Further differentiation of stem cells into the chondrogenic lineage was performed in chondrogenic medium consisting of DMEM high glucose (Invitrogen), $100 \mathrm{nM}$ dexamethasone (Sigma), $100 \mathrm{U} / \mathrm{ml}$ penicillin, $100 \mu \mathrm{g} / \mathrm{ml}$ streptomycin, $0.2 \mathrm{mM} \mathrm{ASAP}$, insulin-transferrin-selenite (ITS+1, Sigma), 100 $\mu \mathrm{g} / \mathrm{ml}$ sodium pyruvate (Sigma), $40 \mu \mathrm{g} / \mathrm{ml}$ proline and $10 \mathrm{ng} / \mathrm{ml}$ TGF $\beta 3$ (Biovision, Mountain View, CA) $[97,99]$.

\section{Gel preparation}

Thiol-functionalized dextran (Dex-SH), based on dextran (31 kDa, Fluka, Buchs, Switzerland), and the crosslinker poly(ethylene glycol) tetra-acrylate (PEG-4-Acr), based on PEG-4 (Mw = 2000, Nektar Therapeutics, Huntsville, AL), were synthesized as described previously [170]. The degree of substitution (DS, defined as the number of substituents per 100 anhydroglucosidic rings of dextran) was either 16 or 22 (the resulting hydrogels are denoted as Dex16-PEG and Dex22-PEG). We prepared gels with a $10 \mathrm{w} / \mathrm{v} \%$ polymer concentration (total dry weight of both dextran and PEG per volume of buffer). Dextran hydrogels were formed via Michael type addition between Dex-SH and PEG-4-Acr in phosphate buffered saline (PBS) at pH 7.2-7.4 at room temperature (Figure 1).

The molar ratio of thiol to unsaturated groups was kept at 1.1, to compensate for thiol groups that have formed disulfide bonds upon exposure to air and to ensure complete reaction with the available acrylate groups. In addition, Dex-SH was weighed and dissolved in PBS under nitrogen flow to reduce contact with $\mathrm{O}_{2}$, which would result in a lower effective concentration of free thiol groups. PEG-4-Acr can crosslink under the influence of light or $\mathrm{O}_{2}$ and was kept in the dark and also weighed under nitrogen flow until mixing of the gel components. To encapsulate cells in the gel, cells were washed with PBS, and the pelleted cells were resuspended in the Dex-SH solution. PEG-4-Acr was mixed thoroughly with Dex-SH by pipetting. A 96-well plate was used as a mold to prepare gels of $150 \mu \mathrm{l}$, with a diameter of $6 \mathrm{~mm}$ and height of $5 \mathrm{~mm}$. As a control, agarose gels were prepared by mixing $0.5 \%$ agarose (Sigma) with PBS. 


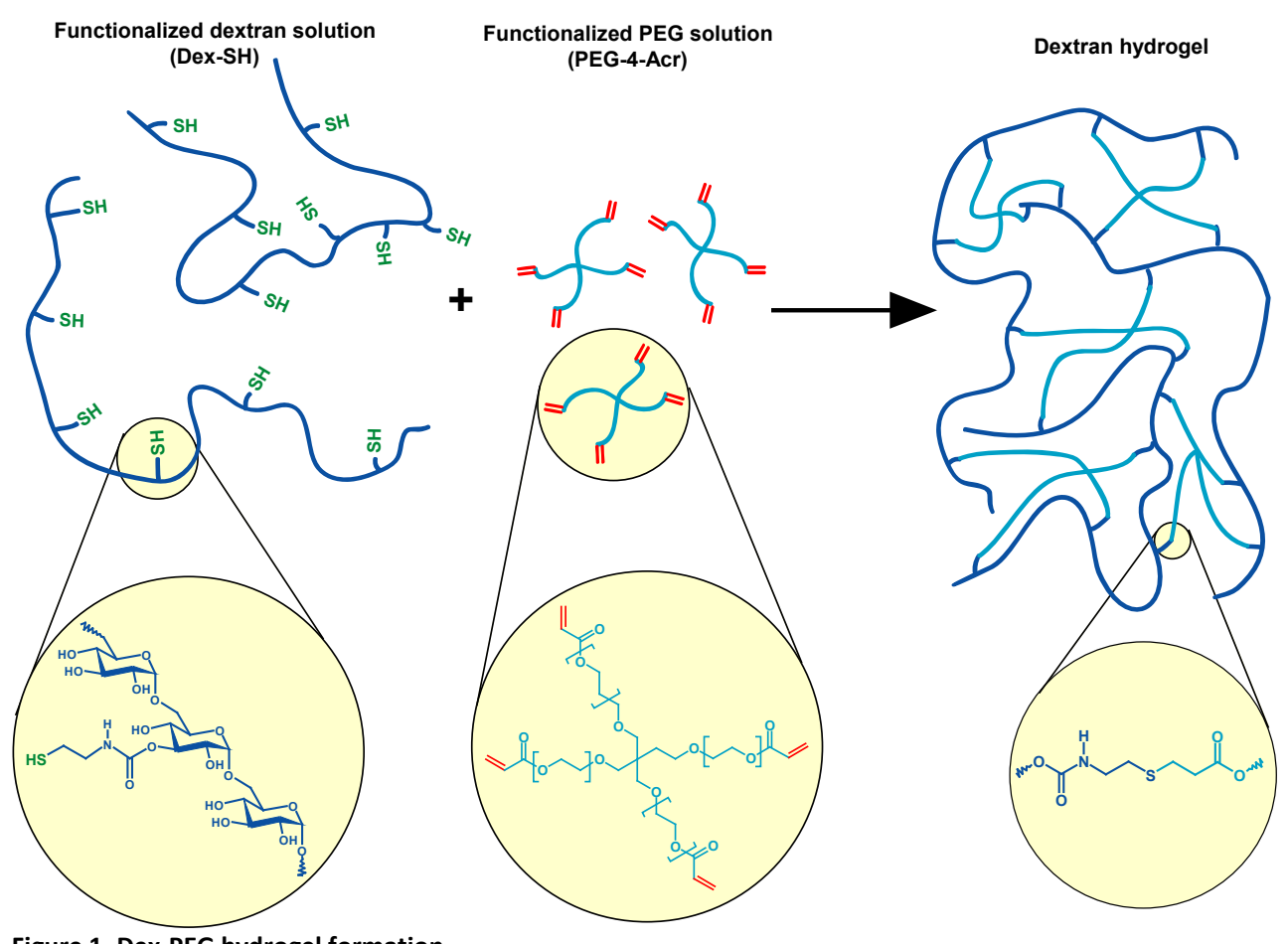

Figure 1. Dex-PEG hydrogel formation

Schematic representation of in situ hydrogel formation by Michael type addition between aqueous solutions of Dex-SH and PEG-4-Acr.

\section{Swelling tests}

Dex16-PEG and Dex22-PEG gels, both with and without chondrocytes, were compared in a swelling test. The swelling experiment was performed in triplicate. After preparation in a mold, the hydrogels were transferred to a well with chondrocyte medium and placed in a $\mathrm{CO}_{2}$ incubator at $37^{\circ} \mathrm{C}$. Medium was refreshed twice a week. The hydrogels were removed from the medium and weighed after 0,5 , $7,11,14,17$ and 21 days. The swelling ratio of the gel was calculated by dividing the weight of the swollen hydrogel after exposure to medium $(W t)$ by the initial hydrogel weight after preparation $\left(W_{0}\right)$ : swelling ratio $=W_{t} / W_{0}$.

\section{Cell viability assay}

A live/dead assay (Molecular Probes, Carlsbad, CA) was used to analyze cell viability according to manufacturer's specifications. Briefly, $1 \mathrm{~mm}$ thick sections were cut from the center of the hydrogels and incubated in PBS solution containing $6 \mu \mathrm{M}$ ethidium homodimer-1 and $2 \mu \mathrm{M}$ calcein AM for 30 minutes at $37^{\circ} \mathrm{C}$. Sections were immediately examined in an inverted fluorescent microscope (Nikon Eclipse E400) using a FITC Texas Red filter. Calcein AM is enzymatically converted, producing green 
fluorescence in living cells. Ethidium homodimer-1 is able to enter cells with damaged membranes and bind to nucleic acids, thereby producing red fluorescence in dead cells.

\section{Histology}

Safranin-O staining was used to analyze tissue morphology and glycosaminoglycan (GAG) expression. Gels were washed with PBS and fixed overnight in $4 \%$ paraformaldehyde (Merck, Darmstadt, Germany) and dehydrated using sequential ethanol series. Hydrogels were embedded in glycol methacrylate (Merck) and cut using a microtome to yield $5 \mu \mathrm{m}$ thick sections. Sections were stained with haematoxylin (Sigma), and fast green (Merck) to visualize cells/cell nuclei (blue staining) and Safranin-O (Sigma) which stained sulphated extracellular GAGs pink.

\section{Quantitative GAG and DNA assay}

Hydrogels for quantitative analysis of GAG expression and cell number were washed with PBS and frozen at $-80^{\circ} \mathrm{C}$. Subsequently, the contents of the gels were digested with $1 \mathrm{mg} / \mathrm{ml}$ proteinase $\mathrm{K}$ (Sigma) in Tris/EDTA buffer (pH 7.6) containing $185 \mathrm{mg} / \mathrm{ml}$ iodoacetamide and $1 \mathrm{mg} / \mathrm{ml}$ pepstatin A (Sigma) for $>16 \mathrm{~h}$ at $56^{\circ} \mathrm{C}$. GAG content was spectrophotometrically determined with 9dimethylmethylene blue chloride (Sigma) staining in PBE buffer $\left(14.2 \mathrm{~g} / \mathrm{l} \mathrm{Na}{ }_{2} \mathrm{HPO}_{4}\right.$ and $3.72 \mathrm{~g} / \mathrm{l}$ $\mathrm{Na}_{2}$ EDTA, pH 6.5) with a microplate reader (Bio-Tek instruments, Richmond, VA) at an absorbance of $520 \mathrm{~nm}$. Values were corrected for background by staining of empty gels. Cell numbers were determined via quantification of total DNA with a CyQuant DNA kit according to the manufacturer description (Molecular Probes) using a fluorescent plate reader (Perkin Elmer, Boston, MA). Data $(n=6$, measured in duplicate) are expressed as mean \pm standard deviation (SD). Statistical significance was determined by one-way ANOVA with SPSS 14.0 software (Chicago, IL).

\section{Results}

\section{Gel formation}

We investigated the potential of Dex-PEG hydrogels for cartilage tissue engineering. To achieve homogeneous seeding in the hydrogel, mixing the cells into the polymer solution before gelation is a prerequisite. In a previous experiment, gelation times of 50 and 22 seconds were determined for Dex16-PEG and Dex22-PEG hydrogels, respectively (data not shown). Therefore, we mixed chondrocytes or ESCS in the Dex-SH solution before adding the crosslinker PEG-4-Acr. The final concentration was 5 million cells $/ \mathrm{ml}$. Aliquots of the Dex-SH solution, with or without cells, were transferred to the mold and a solution of PEG-4-Acr was added by pipetting. Gels formed 
homogeneously within 1 minute, and were transferred to a culture plate with medium using a spatula after hardening for an additional 5 minutes. The presence of cells did not seem to affect the gelation process.

\section{Swelling and degradation of Dex-PEG hydrogels}

Dex-PEG gels are degradable under physiological conditions through hydrolysis of the ester bonds between the thioether and PEG. Upon hydrolysis, the gels swell, until the hydrogel network disintegrates and all the degradation products finally dissolve (Figure $2 \mathrm{~A}$ ).

A

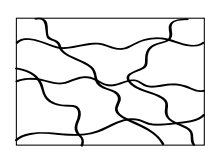

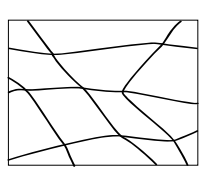
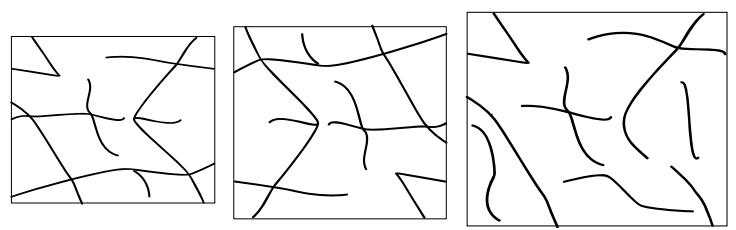

B

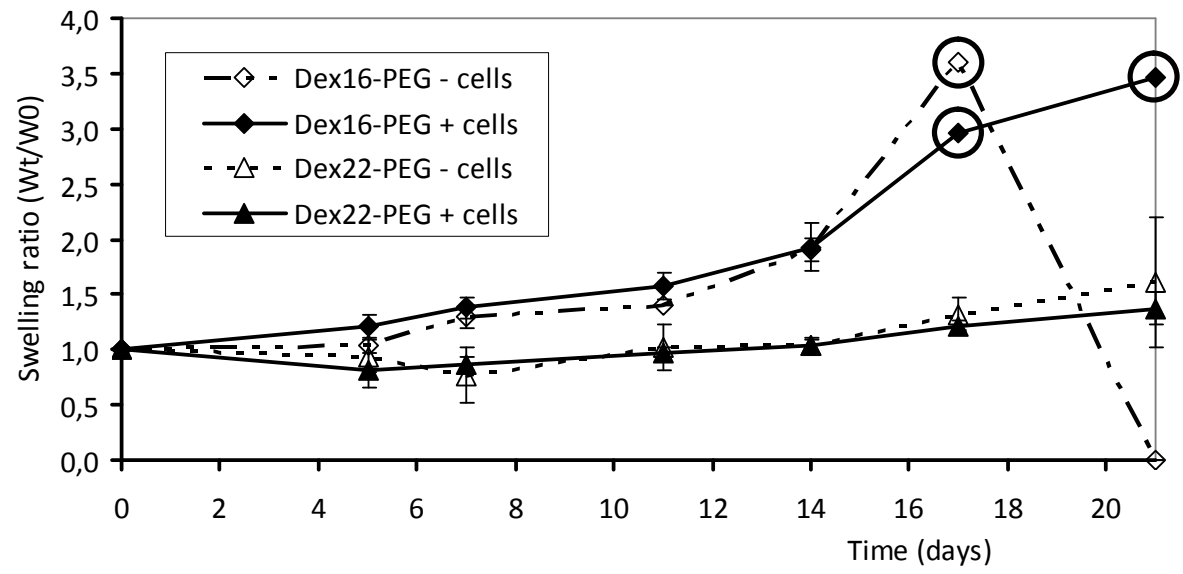

Figure 2. Swelling profiles of Dex16-PEG and Dex22-PEG hydrogels

A) Schematic representation of the swelling and degradation of the hydrogel. B) Hydrogels with two different DS were prepared with and without chondrocytes. Swelling ratios $\left(W_{t} / W_{0}\right)$ are shown as mean $\pm S D(n=3)$. A circle indicates that one or more of the three samples had dissolved completely. Therefore, error bars were omitted for these samples.

The swelling ratio $W_{t} / W_{0}$ was determined by weighing the gels at various time points. The time when no gel was left was taken as the degradation time. Figure $2 \mathrm{~B}$ shows the swelling profiles of the Dex16-PEG and Dex22-PEG gels, both with and without cells. The gels could swell to approximately 3.5 times their original weight $\left(W_{0}\right)$ and the diameter increased from $6 \mathrm{~mm}$ to almost $2 \mathrm{~cm}$. Next, the gels dissolved completely. The gels with a higher DS had an increased degradation time. The Dex16PEG gels degraded in approximately 17 to 22 days. The Dex22-PEG gels degraded slower (Figure 2B) and all gels, both with and without cells, were still present after 21 days. Dex22-PEG gels with cells 
gradually swelled for more than 7 weeks, before they finally went into solution (data not shown). In all experiments, it was observed that the presence of cells in the gel resulted in slower swelling and degradation (Figure 2B).

\section{Viability of chondrocytes in the Dex-PEG hydrogel}

We investigated whether cells would survive the gelation reaction and if in time, sufficient nutrients would diffuse into the gels to maintain the viability of the cells in the center of the gels. The viability of the bovine chondrocytes after 1 h, 1, 2, 7 and 21 days was analyzed. As a control, we encapsulated chondrocytes into a $0.5 \%$ agarose gel. The viability of chondrocytes in both Dex16-PEG and Dex22-PEG gels remained above $95 \%$ at all time points, similar to cells encapsulated in agarose gels (Figure 3).
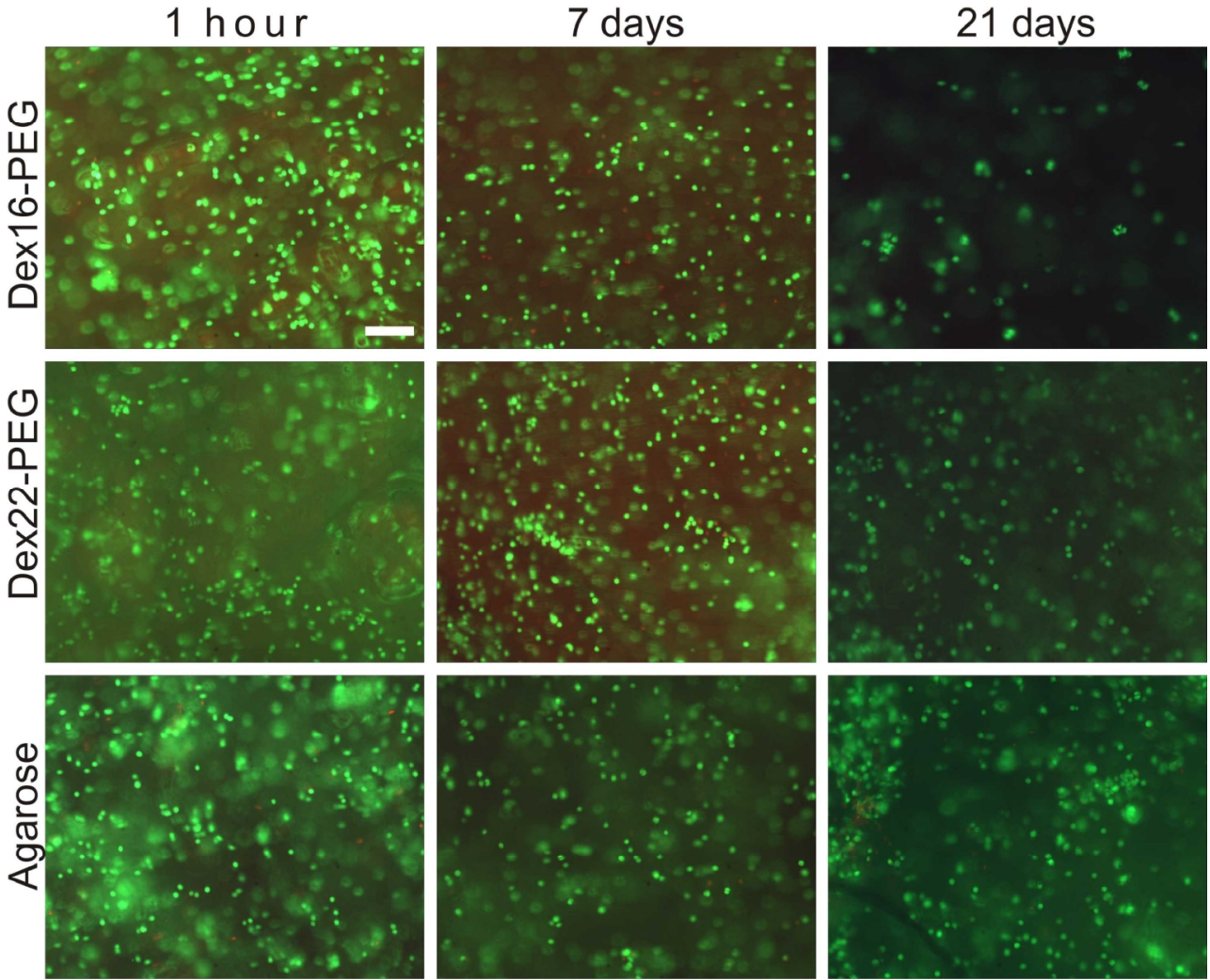

Figure 3. Viability of bovine chondrocytes encapsulated in Dex-PEG hydrogels

The viability of chondrocytes in the hydrogels was measured in time, and compared to the viability of the cells in agarose. Cells are homogeneously distributed in the gels. After 21 days, chondrocytes in the Dex16-PEG gel are more dispersed due to swelling. Green cells indicate viable cells, red cells indicate dead cells. Scale bar represents $100 \mu \mathrm{m}$, all pictures were taken at same magnification. 
Cells were homogeneously distributed in the hydrogels. In time, the Dex-PEG gels took up water and swelled. This resulted in more distance between the chondrocytes, especially observed at day 21 with the Dex16-PEG gel, which was reaching its maximum size, before it would dissolve (Figure 3). Thus, bovine chondrocytes survived both gelation and prolonged culture times in the Dex-PEG hydrogels.

\section{Proliferation and cartilage formation by chondrocytes in Dex-PEG hydrogels}

After 21 days, multicellular aggregates were present in the gels (Figure 4A), although it was more apparent in the agarose samples than in both Dex-PEG gels. This was confirmed by a significant difference in the amount of DNA after 21 days. Two to three times more DNA was measured in the agarose samples than in both Dex-PEG gels (Figure 4B).

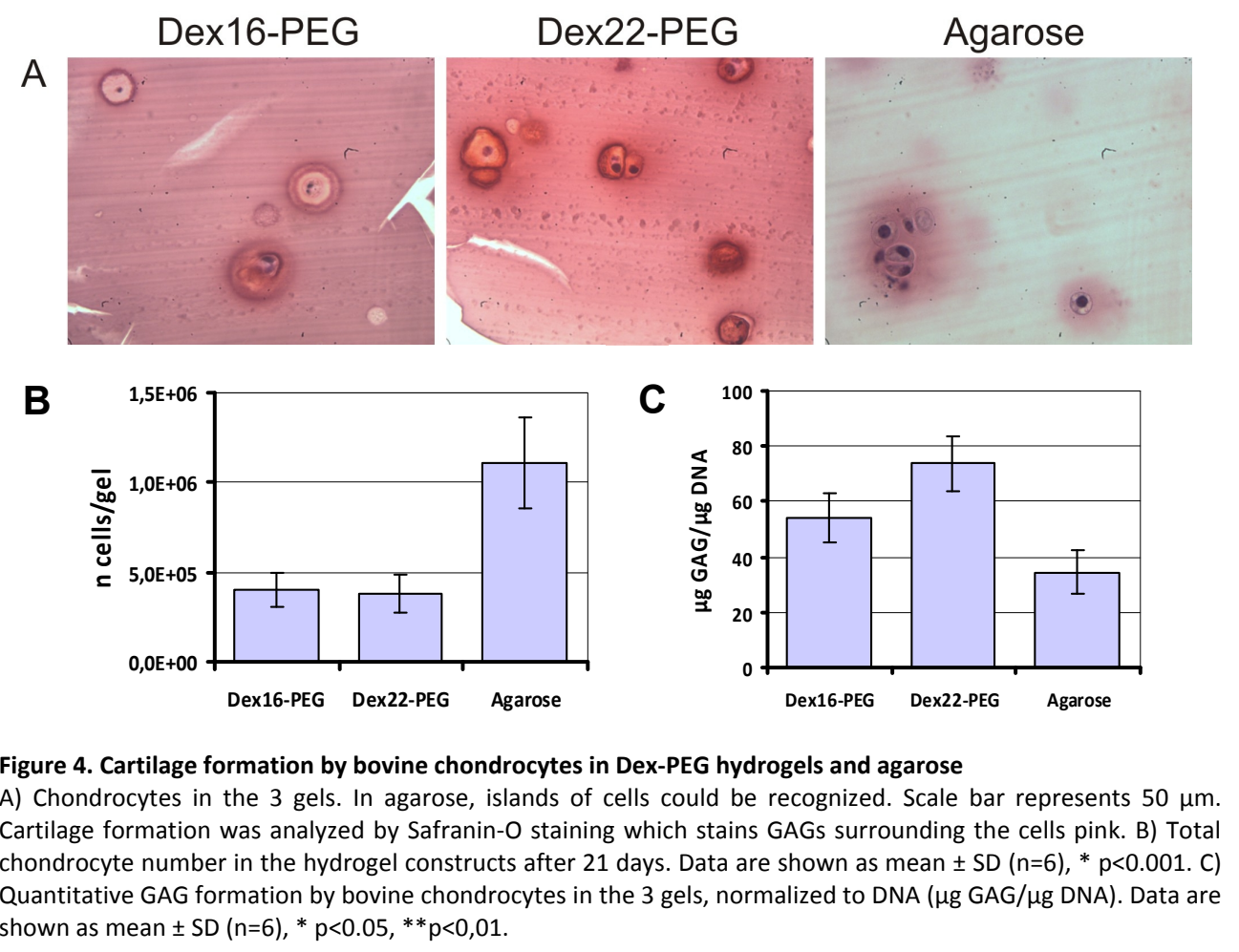

We analyzed glycosaminoglycan (GAG) production both qualitatively and quantitatively as an indication of the formation of cartilage-like tissue by the chondrocytes encapsulated in the hydrogels. During histological processing, the hydrogels shrunk to their original size. As such, we did not observe the spacing of cells as seen in the viability assay samples. Chondrocytes were surrounded by a ring of positively stained GAGs, which diffused into the hydrogel (Figure 4A). The 
highest GAG levels were measured in the agarose samples (data not shown). However, when we corrected GAG production for cell number (GAG/DNA) $(n=6)$, we observed significantly higher levels for both Dex-PEG gels when compared to agarose, and also higher levels for Dex22-PEG than Dex16PEG (Figure 4C).

\section{Viability and cartilage formation by mouse ESCS}

The potential of Dex-PEG hydrogels for cartilage tissue engineering using another chondrogenic cell type, mouse ESCs, was investigated. We first analyzed whether mouse ESCs are able to attach to the hydrogel. The bottom of a well plate was coated with a thin layer of hydrogel. When mouse ESCs in medium were applied on top of the gel, we did not observe any cell attachment (data not shown). On the gel, the mouse ESCs formed embryoid bodies, as also seen when cultured on non-adhering culture plastic. The thin layer of hydrogel degraded fast, and the degradation products in the medium did not interfere with the formation of EBs (data not shown).

Next, the viability of mouse ESCS in the Dex-PEG hydrogel was investigated. Mouse ESCs were encapsulated into the hydrogels using a similar approach as described above for bovine chondrocytes. Two hours after encapsulation, the cells were still viable. Within 2 days, the viability of the cells decreased rapidly (Figure 5A), similar to previous results with mouse ESCs in all other gels we tested so far, such as agarose, alginate, Matrigel and Puramatrix [102]. The viability of intact EBs in the dextran hydrogels was also analyzed. The EBs displayed better survival in the Dex-PEG hydrogels than single EB cells (Figure 5A), as also seen in other hydrogels analyzed [102]. We also analyzed the formation of cartilage tissue by the EBs. We observed some cartilaginous tissue formation by the EBs, both in the Dex16-PEG and Dex22-PEG gels (Figure 5B), indicating that chondrogenic cells other than chondrocytes could also form cartilage tissue in the Dex-PEG hydrogel. 


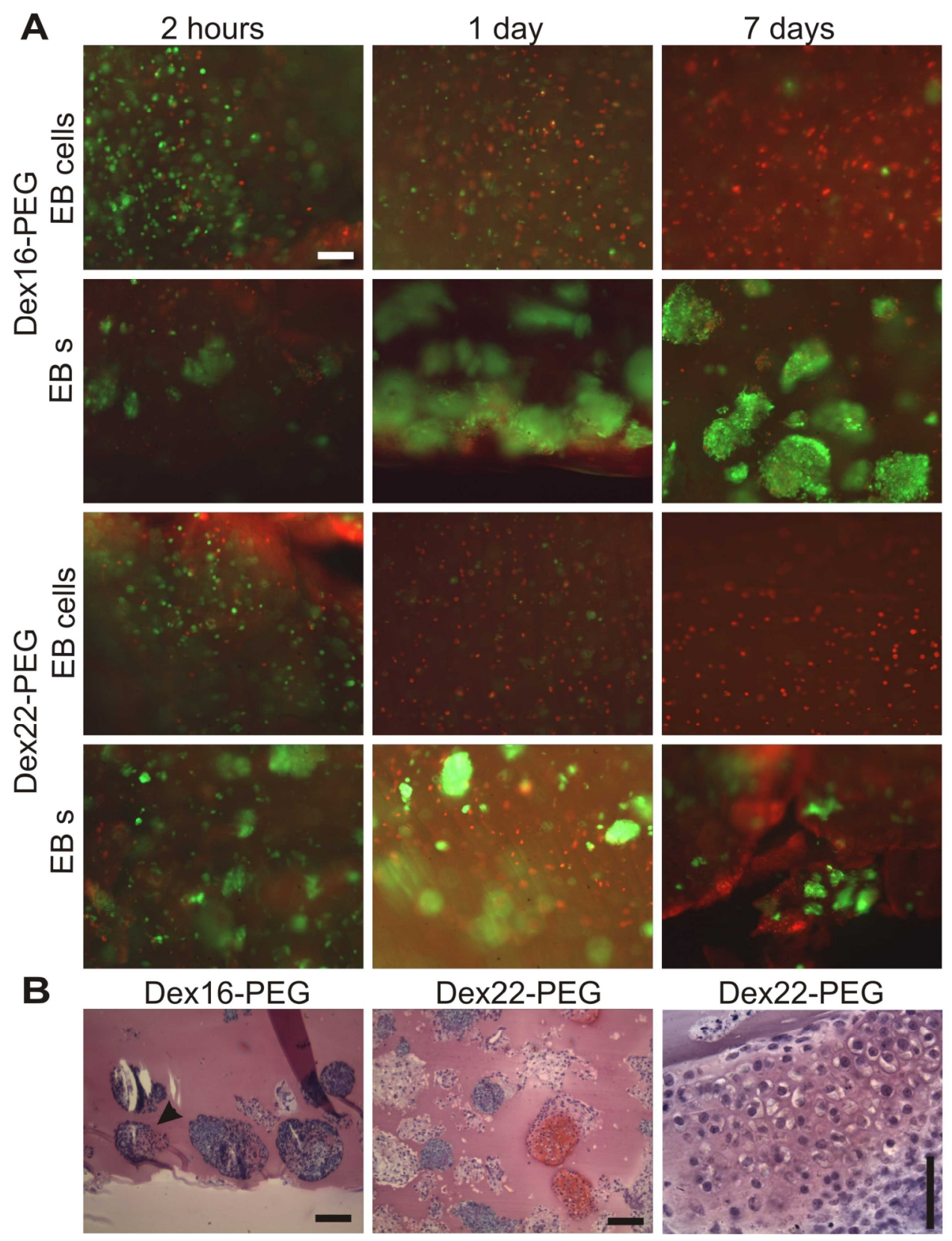

Figure 5. Viability of and cartilage formation by mouse ESCs in Dex-PEG hydrogels

A) Viability of EB cells and intact EBs after 2 hours, 1 and 7 days. EB cells did not survive, intact EBs were still viable. Some red background staining of the gel was observed. Scale bar represents $100 \mu \mathrm{m}$. B) Cartilage formation by intact EBs after 16 days culture in serum-free chondrogenic medium. Pink staining indicates the formation of GAGs by mouse ESC-derived EBs. Horizontal scale bar represents $100 \mu \mathrm{m}$, vertical scale bar represents $50 \mu \mathrm{m}$. 


\section{Discussion}

In this manuscript, the potential of a newly developed dextran-based hydrogel for cartilage tissue engineering was demonstrated. Cells encapsulated in the Dex-PEG hydrogel survived gelation and incubation over several weeks, and formed cartilaginous tissue.

Dex-PEG hydrogels were prepared using thiol-functionalized dextran with a degree of substitution of 16 or 22 and tetra-acrylated star PEG as crosslinker. The hydrogel compositions were chosen based on their gelation time and degradation time. The gelation time should not be too short to allow homogeneous mixing of cells and gelation. On the other hand, too long gelation times would result in settling of the cells at the bottom of the gels, and cell death due to a lack of medium nutrients and longer exposure to reactive groups. The Dex-PEG showed gelation times of 30 to 60 seconds, which might be too fast to add cells after mixing the 2 gel components. Therefore, we decided to mix the cells in the functionalized dextran solution before adding the crosslinker, which resulted in homogeneous cell distribution in the hydrogel and homogeneous gelation.

In some applications, hydrogels are used to deliver the cells into a defect site, and subsequently the gels degrade. However, we wanted a more stable hydrogel in which the cells can first form cartilage tissue. A prerequisite of the design was that the gels had to be stable for at least 3 weeks, to allow less committed stem cells to differentiate into the chondrogenic lineage before the gel degrades. The hydrogels prepared degraded in approximately 3 to 7 weeks. The swelling profile of the Dex-PEG hydrogel was influenced by the DS of dextran. Further fine-tuning of the mechanical and degradation properties can be achieved by varying the molecular weight of the polymer, the concentration of polymer, or choosing another crosslinker $[170,171]$. As such, the hydrogel can be designed for the desired application, for example articular chondrocyte injection. The cells in the hydrogel seem to stabilize the gel, and the degradation time increases accordingly. Whether there is a correlation between cell density, the formed ECM and degradation time has not been determined yet. The in vivo degradation profile of these hydrogels has to be further investigated. The surrounding tissue and confined space in the defect site will be important factors that influence the hydrogel stability and its potential application in tissue engineering.

Chondrocytes are not the only cell source investigated for cartilage tissue engineering. Stem cells are another cell source that upon differentiation into the chondrogenic lineage can form cartilage tissue. Both ESCs [10-12] and adult stem cells, for example mesenchymal stem cells isolated from bone marrow [99] or fat tissue derived from liposuction procedures [177], can form cartilage. We previously investigated the potential of ESCs for cartilage and bone tissue engineering 
$[102,129,178]$. We seeded ESCs in hydrogels and cartilage was successfully formed [102]. Therefore, we also investigated chondrogenic differentiation of mouse ESCs in dextran-based hydrogels. The viability and chondrogenic capacity of mouse ESCs encapsulated in the Dex-PEG gels was investigated first. Cell death was observed when single EB cells were encapsulated in the hydrogel. Similar results were obtained with all gels we have investigated earlier [102]. However, intact EBs did survive the encapsulation and subsequent incubation period, and formed cartilaginous tissue. Cartilage formation was mainly observed in the EBs, which is comparable to the behavior of EBs in other non-adhering gels such as agarose and alginate [102]. In gels to which cells could adhere, such as Matrigel or Puramatrix, a more intense superficial layer of cartilage was observed, not only restricted to the EBs [102]. Further functionalization of the Dex-PEG hydrogel by adding adhesion molecules might enhance cartilage formation by ESCs. In contrast, the non-adhesive property of the dextran-based hydrogels may benefit tissue engineering using articular chondrocytes. We previously observed that the integrin-mediated attachment of chondrocytes to polymeric films is associated with dedifferentiation of the chondrocytes and reduced cartilage formation [179]. It is important to functionalize the hydrogel for the appropriate cell type with the appropriate adhesion molecules. When bovine chondrocytes were seeded in the hydrogel, cartilaginous tissue was formed. Even though the total amount of GAG was less than in control agarose samples, the amount of GAG per cell was higher than in agarose. Therefore, we conclude that there is potential for Dex-PEG hydrogels for tissue engineering applications. Future work will include additional biocompatibility tests and in vivo performance of the gel.

\section{Acknowledgements}

JMJ was supported by Dutch Technology Foundation (STW) grant TPG 5923, CH was funded by the Netherlands Organization for Scientific Research (NWO), JdB was supported by a Senter/Novem grant.

We would like to thank Weyl Beef Products in Enschede for generously supplying us with calf and bovine cartilage. 



\title{
CHAPTER 4 CRITICAL STEPS TOWARD A
}

\section{TISSUE-ENGINEERED CARTILAGE IMPLANT USING EMBRYONIC STEM CELLS}

\author{
Jojanneke M. Jukes, Lorenzo Moroni, Clemens A. van Blitterswijk, Jan de Boer \\ Institute for Biomedical Technology, Department of Tissue Regeneration, \\ University of Twente, Enschede, The Netherlands
}

Pink - it's my new obsession

Pink - Aerosmith - Nine lives 


\title{
CRITICAL STEPS TOWARD A TISSUE-ENGINEERED CARTILAGE IMPLANT USING EMBRYONIC STEM CELLS
}

\begin{abstract}
Embryonic stem cells (ESCs) cells are a potential source for cartilage tissue engineering because they provide an unlimited supply of cells, which can be differentiated into chondrocytes. So far, chondrogenic differentiation of both mouse and human ESCs has only been demonstrated in 2D cultures, in pellet cultures, in a hydrogel or on thin biomaterials. The next challenge will be to form cartilage on a load-bearing, clinically relevant sized scaffold in vitro and in vivo, to regenerate defects in patients suffering from articular cartilage disorders. For a successful implant, cells have to be seeded efficiently and homogenously throughout the scaffold. Parameters investigated were the scaffold architecture, seeding method and cellular condition. Seeding in a 3D fiber deposited scaffold was more homogenous than in a compression moulded scaffold. The seeding efficiency on bare scaffolds was compromised by the absence of serum in the chondrogenic medium, but could be improved by combining the cells with a gel and subsequent injection into the 3DF scaffolds. However, the viability of the cells was unsatisfactory in the interior of the graft. Cell aggregates, so called embryoid bodies, were seeded with increased survival rate. Mouse ESCs readily underwent chondrogenic differentiation in vitro in pellets, on bare scaffolds, in Matrigel and in agarose, both as single cells and in embryoid bodies. The differentiation protocol requires further improvement to achieve homogenous differentiation and abolish teratoma formation in vivo. We conclude that embryonic stem cells can be used as a cell source for cartilage tissue engineering, pending further optimization of the strategy.
\end{abstract}




\section{Introduction}

Articular cartilage has a limited capacity for self-repair after injury. Therefore, various surgical procedures have been developed to repair damaged cartilage. A relatively new approach is cartilage tissue engineering, for example articular chondrocyte transplantation (ACT) [64]. In cartilage tissue engineering, chondrogenic cells, such as articular chondrocytes $[180,181]$ or mesenchymal stem cells, can be combined with a porous biodegradable polymeric scaffold, to create a clinically relevant sized, load bearing implant. Our lab has used copolymers of soft, hydrophilic poly(ethylene oxide terephthalate) and hard, hydrophobic poly(butylene terephthalate) (PEOT/PBT) as a potential polymer for articular cartilage repair. By varying the molecular weight of the poly(ethylene glycol) segments and the PEOT/PBT weight ratio, an entire family of polymers was obtained, each with its own mechanical properties [181-183]. Furthermore, by novel 3D fiber deposition technology, the architecture, pore size and shape of the PEOT/PBT scaffolds can be varied in a reproducible manner, by which structures that closely mimic the biomechanical properties of cartilage can be produced [181,182].

The recent surge in embryonic stem cell research has attracted the interest of regenerative medicine and tissue engineering. ESCs are capable of indefinite, undifferentiated proliferation in vitro while maintaining the potential to differentiate into derivatives of all three embryonic germ layers: endoderm, mesoderm, and ectoderm [10-12]. In essence, ESCs can provide an unlimited supply of cells which can be differentiated into chondrocytes, under the right differentiation conditions.

A number of papers have been published about chondrogenic differentiation of mouse ESCs. Starting point of these differentiation studies was the formation of embryoid bodies (EBs). EBs are aggregates of randomly differentiating ESCs, which form spontaneously in suspension culture. Kramer et al. [87] found cartilaginous regions in the outgrowths of plated embryoid bodies, when cultured in basic proliferation medium. By adding BMP2 or BMP4 to the culture medium, they increased the number of cartilaginous nodules per embryoid body, whereas TGFß1 resulted in a slight decrease of alcian blue-positive nodules. In studies by Kawaguchi et al. [93], BMP4 was not sufficient to induce chondrogenesis. However, retinoic acid treatment of the EBs followed by culture in basic proliferation medium to which TGFß3 and parathyroid hormone were added did induce chondrogenesis. Zur Nieden et al. [94] analysed the addition of several supplements to the basic culture medium during embryoid body formation and during the attached culture. When BMP2 and TGFß1 were added during EB formation and insulin, ascorbic acid and BMP2 were added during the further differentiation, the most profound up-regulation of cartilage-specific genes was seen. The 
above-mentioned studies used a 2D differentiation protocol. Tanaka et al. [98] cultured EBs encapsulated in alginate, and EB cells in micromass and pellet cultures, thus taking the step towards a 3D differentiation protocol. Chondrogenesis was not enhanced in the encapsulated EBs, when compared with plated EBs. However, culturing EB cells in micromass or pellet cultures significantly increased the expression of cartilage-specific markers. Nakayama et al. [101] took the differentiation protocol another step forward and selected a mesodermal fraction from the EB cells and subjected these cells to pellet culture in serum-free chondrogenic medium containing TGFß3 as described earlier for mesenchymal stem cells [97,99]. By varying the combination of TGFß3, BMP4 and PDGF$\mathrm{BB}$ in the chondrogenic medium, the cartilaginous tissue became more homogeneous. Recently, chondrogenic differentiation of ESCs has been investigated in a tissue engineering context. Hwang et al. encapsulated embryoid bodies in a hydrogel and cultured them in chondrogenic medium. Again it was shown that a 3D environment is beneficial for chondrogenesis [111]. Chondrogenic differentiation of human ESCs has only been described by Levenberg et al. Cells were seeded on 1 $\mathrm{mm}$ thin porous polymer scaffold sections and cartilaginous tissue was formed by adding TGFß1 to the culture medium [36].

So far, chondrogenic differentiation of ESCs has been accomplished in 2D cultures or in micromass or pellets cultures, encapsulated in a hydrogel or on thin biomaterials. For tissue engineering applications, a load-bearing, clinically relevant sized scaffold is desirable. To meet this, we investigated the chondrogenic potential of mouse ESCs on $4 \mathrm{~mm}$ thick PEOT/PBT scaffolds.

\section{Materials and methods}

\section{Mouse ESC culture}

Mouse ESC line iB10 was cultured as described previously [176]. In brief, cells were plated at a density of 5000-10000 cells $/ \mathrm{cm}^{2}$ on gelatin-coated tissue culture flasks. Mouse ESCs were cultured in $50 \%$ mouse ESC proliferation medium consisting of Dulbecco's Modified Eagle's Medium (DMEM, Biowhittaker) containing $4.5 \mathrm{mg} / \mathrm{ml} \mathrm{D-glucose,} \mathrm{10 \%} \mathrm{fetal} \mathrm{bovine} \mathrm{serum} \mathrm{(selected} \mathrm{batch} \mathrm{for} \mathrm{mouse}$ ESC culture, Greiner), $0.1 \mathrm{mM}$ non-essential amino acids (NEAA, Sigma), $4 \mathrm{mM}$ L-glutamine (Invitrogen), $100 \mathrm{U} / \mathrm{ml}$ penicillin (Invitrogen), $100 \mu \mathrm{g} / \mathrm{ml}$ streptomycin (Invitrogen) and $50 \%$ of Buffalo rat liver cell-conditioned mouse ESC proliferation medium [176]. Prior to use $1000 \mathrm{U} / \mathrm{ml}$ Leukemia Inhibitory Factor (Esgro, Chemicon International) and $50 \mu \mathrm{M}$ 2-mercapto-ethanol (Gibco) were added to the medium. Cells were grown at $37^{\circ} \mathrm{C}$ in a humidified $5 \% \mathrm{CO}_{2}$ incubator and passaged with $0.05 \%$ trypsin/EDTA before reaching confluence. 


\section{Scaffolds}

PEOT/PBT block copolymers were obtained from IsoTis S.A. (Bilthoven, The Netherlands). The molecular weight of the starting poly(ethylene glycol) (PEG) segments used in the polymerization process was $300 \mathrm{~g} / \mathrm{mol}$, while the weight ratio between PEOT and PBT blocks was 55 and 45 respectively (300PEOT55PBT45). Compression moulded (CM) scaffolds were prepared using a compression moulding and particle leaching technique, as described previously [184]. Briefly, 300PEOT55PBT45 granules were mixed with 75 vol\% sodium chloride grains with a size ranging from 400-600 $\mu \mathrm{m}$. After compression moulding under heat and pressure $\left(\mathrm{T}=230^{\circ} \mathrm{C}, \mathrm{P}=2.8 \mathrm{MPa}\right)$, the 300PEOT55PBT45 block was immersed in demineralised water to remove the sodium chloride, and dried under reduced pressure in a vacuum oven. 3D fiber deposited (3DF) scaffolds were fabricated with a Bioplotter device (Envisiontec $\mathrm{GmbH}$, Germany), essentially an XYZ plotter construction as previously described by Moroni et al. [182]. The plotted fibers had a diameter of $170 \mu \mathrm{m}$, while the fiber spacing was set to $600 \mu \mathrm{m}$, and the layer thickness was set to $150 \mu \mathrm{m}$. The angle between two successive layers was $90^{\circ}$. For scaffolds with a larger pore volume, the fiber diameter was $275 \mu \mathrm{m}$, the layer thickness was set to $225 \mu \mathrm{m}$ and the fiber spacing was set to $1200 \mu \mathrm{m}$ and the fiber orientation was changed after two printed layers $\left(0^{\circ} / 0^{\circ} / 90^{\circ} / 90^{\circ}\right)$. Cylindrical scaffolds with a diameter of 4 or $5 \mathrm{~mm}$ were cored out from $4 \mathrm{~mm}$ thick porous CM and 3DF blocks, and placed in a cylindrical, radio-frequency based glow-discharge glass bell chamber (Harrik Scientific Corp, NY). A vacuum was applied to the chamber $(0.01 \mathrm{mbar})$ and subsequently flushed 4 times with argon (Hoekloos B.V., The Netherlands). The scaffolds were then treated under argon plasma for 30 minutes [185].

\section{Differentiation}

To initiate differentiation, embryoid bodies (EBs) were formed by culturing mouse ESCs at 20,000 cells $/ \mathrm{cm}^{2}$ in non tissue culture-treated 6-well plates (Greiner) in $4 \mathrm{ml} \mathrm{EB}$ medium (DMEM, 10\% FBS, $0.1 \mathrm{mM}$ non-essential amino acids, $4 \mathrm{mM}$ L-glutamine, $100 \mathrm{U} / \mathrm{ml}$ penicillin, $100 \mu \mathrm{g} / \mathrm{ml}$ streptomycin and $50 \mu \mathrm{M}$ 2-mercaptoethanol) for 4 days. To obtain a single cell suspension, EBs were dissociated with $0.25 \%$ trypsin/EDTA. Further differentiation into the chondrogenic lineage was performed in chondrogenic differentiation medium (diff+) consisting of DMEM high glucose (Gibco), $100 \mathrm{nM}$ dexamethason (Sigma), $100 \mathrm{U} / \mathrm{ml}$ penicillin, $100 \mu \mathrm{g} / \mathrm{ml}$ streptomycin, insulin-transferrin-selenite (ITS+1, Sigma), $0.2 \mathrm{mM}$ ascorbic acid 2-phosphate (Sigma), $100 \mu \mathrm{g} / \mathrm{ml}$ sodium pyruvate (Sigma), 40 $\mu \mathrm{g} / \mathrm{ml}$ proline (Sigma) and $10 \mathrm{ng} / \mathrm{ml}$ TGF $\beta 3$ (R\&D Systems or Biovision) $[97,99]$. For pellet culture, aliquots of $0.5 \times 10^{6} \mathrm{~EB}$ cells in $1 \mathrm{ml}$ medium were centrifuged at $500 \times \mathrm{g}$ for 2 minutes in $12 \mathrm{ml}$ 
polypropylene conical tubes, as described for mesenchymal stem cells $[97,99]$.The medium was changed every 3 to 4 days.

For direct seeding studies, argon-plasma-treated scaffolds were preincubated with EB medium for 2 $\mathrm{h}$ to allow serum proteins to attach to the scaffold to improve cell attachment. For static seeding, scaffolds were seeded with $2.5 \times 10^{6}$ EB cells in $100 \mu \mathrm{lEB}$ medium, and after 2 hours $2 \mathrm{ml}$ EB medium was added. For dynamic seeding, $5 \times 10^{6}$ cells per scaffold were seeded in a $30 \mathrm{ml}$ spinner flask stirred at $50 \mathrm{rpm}$. For dynamic seeding in a small volume, $2 \mathrm{ml}$ tubes with a lid with a membrane, which permits gas exchange (LidBac, Eppendorf) were used. The scaffolds were placed in the tube, and $2.5 \times 10^{6} \mathrm{~EB}$ cells or intact EBs in $2 \mathrm{ml}$ EB medium were added. The tubes were placed in a cylindrical tube holder and rotated on a roller bank overnight, after which the scaffolds were transferred to a 6well plate. After static or dynamic seeding overnight, the medium was replaced by chondrogenic or control (EB) medium and subsequently cultured statically for 21 days.

For combined gel-scaffold experiments, scaffolds were placed in a $6 \mathrm{~mm}$ cylindrical mould. A cell suspension of $10 \times 10^{6} \mathrm{~EB}$ cells or EBs (equivalent of $10 \times 10^{6}$ cells) in $100 \mu \mathrm{l}$ growth factor reduced Matrigel (BD Biosciences) or agarose was seeded onto the scaffold and incubated for $30 \mathrm{~min}$ at $37^{\circ} \mathrm{C}$ or room temperature to allow solidification of the gel. EBs were seeded onto scaffolds with large pores, to allow better infiltration of the EBs-gel mixture. After gel formation, the constructs were detached from the mould and transferred to a 6-well plate. Chondrogenic or control medium was replaced twice a week.

\section{Quantitative $P C R$}

Three pellets were combined for qPCR reactions. Total RNA was isolated using an RNeasy mini kit (Qiagen) and on-column DNase-treated with 10 units RNase-free DNase I (Gibco) at $37^{\circ} \mathrm{C}$ for 30 minutes. DNase was inactivated at $72^{\circ} \mathrm{C}$ for 15 minutes. The quality and quantity of RNA was analysed by spectrophotometry. Two $\mu \mathrm{g}$ of RNA was used for first strand cDNA synthesis using Superscript II (Invitrogen) according to the manufacturers' protocol. One $\mu$ l of 100x diluted cDNA was used for GAPDH control amplification and $1 \mu \mathrm{l}$ of undiluted CDNA was used for other genes. QPCR was performed on a Light Cycler real-time PCR machine (Roche) using the SYBR Green I master mix (Invitrogen). Data was analysed using Light Cycler software version 3.5.3, using the fit point method by setting the noise band to the exponential phase of the reaction to exclude background fluorescence. Expression of chondrogenic genes was calculated relative to GAPDH levels by comparative $\Delta \mathrm{C}_{\mathrm{T}}$ method [186]. Primer sequences are listed in Table 1. 


\begin{tabular}{|c|c|c|c|c|}
\hline Gene & & Sequence & Product & Ref or GenBank \\
\hline \multirow[t]{2}{*}{ Collagen type II } & fwd & 5'-CAAGGCCCCCGAGGTGACAAA-3' & $216 \mathrm{bp}$ & NM_031163 \\
\hline & rev & 5'-GGGGCCAGGGATTCCATTAGAGC-3' & & \\
\hline \multirow[t]{2}{*}{ Collagen type I } & fwd & 5'-GCATGGCCAAGAAGACATCC-3' & $83 \mathrm{bp}$ & [142] \\
\hline & rev & 5'-CCTCGGGTTTCCACGTCTC-3' & & \\
\hline \multirow[t]{2}{*}{ COMP } & fwd & 5'CGAAACGTGGGCTGGAAGGATAAA-3' & 255 bp & NM_016685 \\
\hline & rev & 5'-TCTCTGCAGCCCGGTGACTCTCGTA-3' & & \\
\hline \multirow[t]{2}{*}{ GAPDH } & fwd & 5'-AACGACCCCTTCATTGAC-3' & $191 \mathrm{bp}$ & [187] \\
\hline & rev & 5'-TCCACGACATACTCAGCAC-3' & & \\
\hline
\end{tabular}

\section{Cell viability assay}

A live/dead assay (Molecular Probes) was used to analyze cell viability according to manufacturer's specifications. Briefly, $1 \mathrm{~mm}$ thick sections were cut from the center of the scaffolds and incubated in PBS solution containing $6 \mu \mathrm{M}$ ethidium homodimer-1 and $2 \mu \mathrm{M}$ calcein AM for 30 minutes at $37^{\circ} \mathrm{C}$. Sections were immediately examined in an inverted fluorescent microscope (Nikon Eclipse E400) using a FITC Texas Red filter. Calcein AM is enzymatically converted, producing green fluorescence in living cells. Ethidium homodimer-1 is able to enter cells with damaged membranes and bind to nucleic acids, thereby producing red fluorescence in dead cells.

\section{Cell quantitation}

Quantitation of total DNA was performed by Cyquant Cell Proliferation assay kit (Invitrogen) using a spectrofluorometer (Perkin Elmer, IL). DNA content was translated into cell numbers by normalizing to a cell and DNA standard. Six samples were analyzed per condition.

\section{Histology}

Safranin-O staining was used to analyze tissue morphology and glycosaminoglycan (GAG) expression. Constructs were fixed in $0.14 \mathrm{M}$ cacodylate buffer $(\mathrm{pH} 7.2-7.4)$ containing $0.25 \%$ glutaraldehyde (Merck) and dehydrated using sequential ethanol series. Pellets and 300/55/45-gel constructs were plastic embedded in glycol methacrylate (Merck) and cut using a microtome to yield $5 \mu \mathrm{m}$ thick sections. Sections were stained with haematoxylin (Sigma), and fast green (Merck) to visualize cells/cell nuclei (blue staining) and Safranin-O (Sigma), which stained sulphated extracellular GAGs pink. 


\section{Immunohistochemistry}

Samples were embedded in cryomatrix (Thermo Shandon) and cut on a cryotome (Thermo Shandon) to yield $5 \mu \mathrm{m}$ sections. Cryosections were fixed in ice-cold acetone for 5 minutes and incubated for 1 hour in blocking buffer (PBS supplemented with 10\% FBS). Subsequently, the samples were treated with monoclonal antibody for collagen type II (1:100, II-II6B3, Developmental Studies Hybridoma Bank). After 1 hour, samples were washed with PBS and incubated with the secondary goat antimouse antibody (1:100, DAKO) for 1 hour. Staining was visualized using DAB-solution (DAKO) for 5 minutes and haematoxylin as a counter stain for 3 minutes.

\section{In vivo studies}

For in vivo studies, samples were first precultured in vitro in serum-free chondrogenic medium with (Matrigel samples) or without (agarose samples) TGF $\beta 3$ for 21 days. Before implantation, samples were washed in PBS. Nude mice (HsdCpb:NMRI-nu, Harlan) were injected with buprenorphine (Temgesic) for pain relief and were kept under isoflurane anastehesia during surgery. The surgical site was cleaned with ethanol, 4 subcutaneous pockets were created on the lateral sides of the spine at the back of the mouse. In each pocket, 1 construct was inserted. After 21 days, animals were sacrificed and the implants were removed and processed histologically as described above. Animals were housed at the Central Laboratory Animal Institute (Utrecht University, The Netherlands) and experiments were approved by the local Animal Care and Use committee.

\section{Results}

\section{Chondrogenic differentiation of mouse ESCs in a pellet culture}

We first assessed the chondrogenic potential of the mouse ESC line iB10. A common first step in differentiation protocols of ESCs is the formation of embryoid bodies (EBs). For chondrogenic differentiation, we used the well-established pellet culture system [97,100]. 4-day old EBs were dissociated into a single cell suspension, pelleted at a density of 500,000 cells and cultured in serumfree chondrogenic differentiation medium with or without TGF $\beta 3$ (diff+ or diff-). As a control, pellets were also cultured in EB medium. Within 24 hours after incubation, most cells condensated into a spherical aggregate (not shown).

After 14 days of culture in both complete and incomplete chondrogenic medium, Safranin-Opositive nodules were observed in the pellets, indicative of chondrogenic differentiation (data not shown), which was even more pronounced after 21 days (Figure 1A). The cartilaginous nodules contained large cells in lacunae surrounded by extracellular matrix containing glycosaminoglycans 
and collagen type II, all of which are characteristics of cartilage tissue. It should be noted however that we never observed homogeneous staining of either markers, indicating that other cell types were present in the pellets. In contrast, we never observed GAG or collagen type II expression in cells cultured in control medium.

A

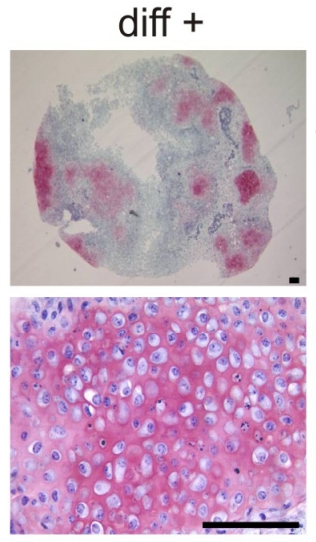

Saf-O

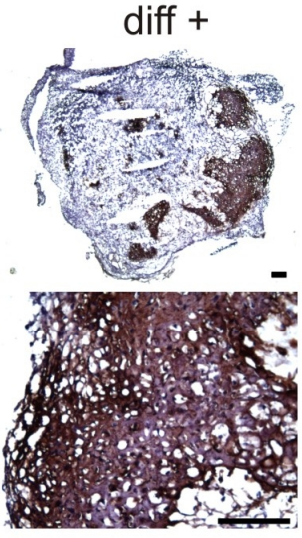

Col2 diff -
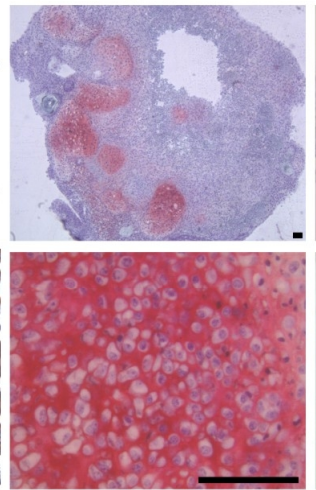

Saf-O ctrl

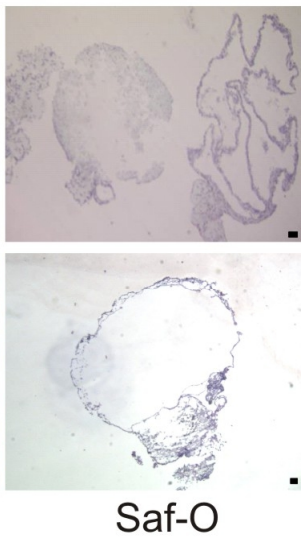

B

Col2

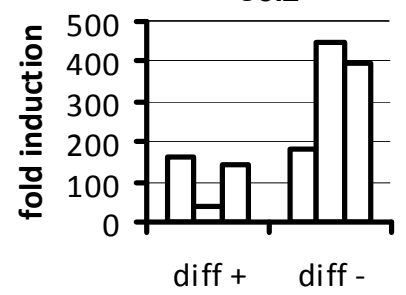

COMP

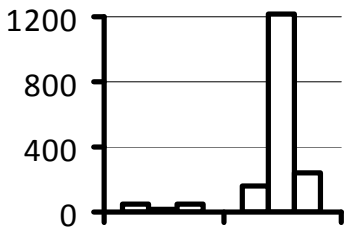

diff + diff -
Col1

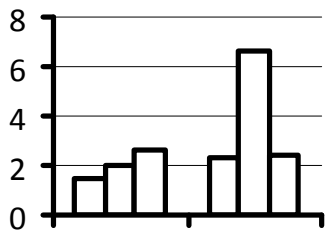

diff + diff -

Figure 1. Chondrogenic differentiation of mouse ESCs in a pellet culture

A) Representative pictures of histological sections of mouse ESC pellets cultured in complete chondrogenic medium with TGFß3 (diff+), incomplete chondrogenic medium without TGFß3 (diff-) or control (ctrl) medium for 21 days. Pink Safranin-O (Saf-O) staining of proteoglycans indicates cartilage formation. Immunostaining for collagen type II (Col2) is visualized by brown staining. Higher magnification view of the samples cultured in chondrogenic medium shows typical cartilage morphology. Scale bar: $100 \mu \mathrm{m}$. B) Collagen type II, cartilage oligomeric matrix protein and collagen type I expression pellets of mouse ESCs, determined by qPCR. Pellets were cultured in complete chondrogenic (diff+) or incomplete chondrogenic (diff-) medium for 21 days. Levels are normalized to pellets grown in control medium. Every bar indicates the expression in 3 pooled pellets.

For further verification of chondrogenic differentiation in pellet cultures, we performed $\mathrm{qPCR}$ analysis on a number of well-known cartilage-specific genes. After 21 days, we found a substantial up-regulation of collagen type II and cartilage oligomeric matrix protein (COMP) expression in chondrogenic medium as compared to pellets in control medium (Figure 1B). To illustrate the expression of collagen type II in the pellets, we plotted the samples separately. We also investigated the expression of the osteogenic differentiation marker collagen type I. Some up-regulation of 
collagen type I was observed, but not in the same order of magnitude as the up-regulation of the chondrogenic markers (Figure 1B). When pellets were cultured in incomplete chondrogenic medium, which lacks TGFß3, the same trend was seen. The absence of TGFß3 in the differentiation medium does not seem to result in lower expression of chondrogenic genes (Figure 1B) and histological data confirmed the presence of cartilage like tissue (Figure 1A). The histological and qPCR analysis demonstrate that ESCs efficiently undergo chondrogenic differentiation.

\section{Seeding and chondrogenic differentiation of mouse ESCS on bare scaffolds}

Since pellets have a diameter of 1-2 $\mathrm{mm}$, these cannot be used to heal large cartilage defects. Therefore, mouse ESCs were seeded onto porous polymeric scaffolds and their chondrogenic potential was investigated. Cylindrical scaffolds with a diameter and thickness of $4 \mathrm{~mm}$ were prepared by either compression moulding (CM) or 3D fiber deposition technology (see Figure 2A). For a successful implant, cells have to be efficiently seeded throughout the scaffold.

First, 4-day old EB cells were statically seeded onto CM scaffolds, both in chondrogenic and control medium. Cell staining showed that EB cells did not distribute throughout the scaffold and mainly attached on the outside of the scaffold (not shown). Consequently, after 21 days in chondrogenic medium, cartilaginous regions were only present on the outside of the scaffolds, as indicated by Safranin-O staining, whereas no cells were seen inside the scaffolds (Figure 2B). Next, we designed fully interconnected porous scaffolds by 3D fiber deposition, as described previously $[181,182]$. Static seeding and culturing of EB cells and EBs (Figure $2 \mathrm{C}$ ) resulted in inhomogeneous cell distribution as a result of gravitational forces.

The homogeneity was improved by dynamic seeding in a spinner flask (Figure 2C). Quantification of cell seeding indicates that static seeding is more efficient than dynamic seeding (Figure 2D). However, we feel that the efficiency of seeding does not outweigh the inhomogeneity observed by light microscopy (Figure 2C) and we anticipated that dynamic seeding at high cell densities might overcome both problems.

Indeed, dynamic, high cell density seeding increased seeding efficiency. We used $2 \mathrm{ml}$ filter-tubes on a roller bank instead of the large spinner flasks. Both EB cells and intact EBs were dynamically seeded overnight, to allow attachment and aggregation of cells onto the 3DF scaffolds. For EB cells, the seeding efficiency in the tube was higher than in the spinner flask, but still not high enough to cover all available surface area (data not shown). More EBs attached to the scaffold, but the cell distribution was less homogeneous, due to random aggregation of EBs inside the scaffolds pores (Figure 2C). 

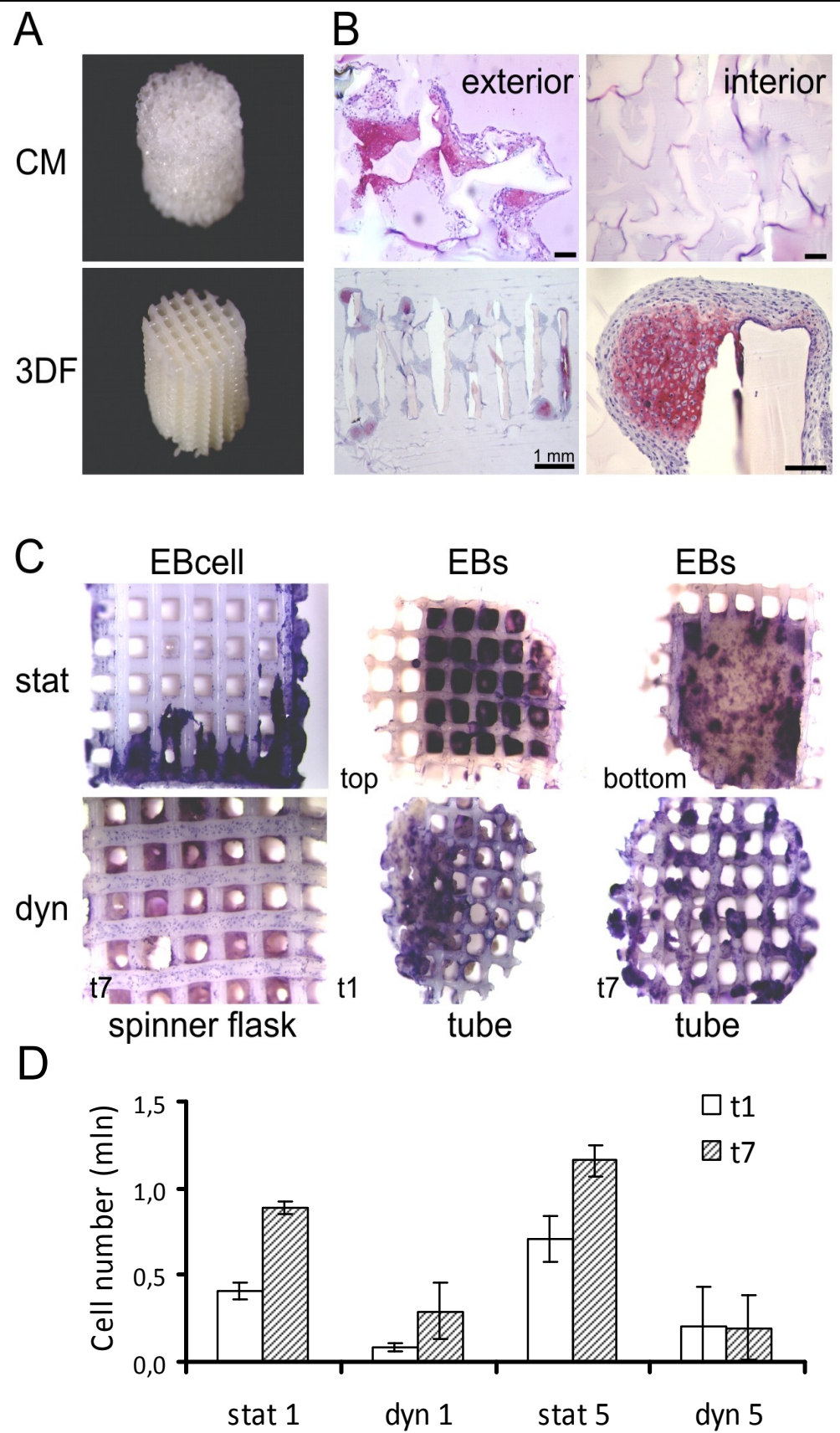

Figure 2. Chondrogenic differentiation of mouse ESCs on scaffolds

A) Macroscopic image of a cylindrical $4 \times 4 \mathrm{~mm}$ compression-moulded (CM) and 3D fiber deposited (3DF) scaffold. B) Safranin-O stained histological sections of mouse ESCs cultured statically in chondrogenic medium (diff+) for 21 days on CM (top) or 3DF (bottom) scaffolds. Pink Safranin-O staining of GAGs is observed on both scaffolds. Note the absence of cells on the interior of CM scaffolds. Scale bar: $100 \mu \mathrm{m}$, unless stated otherwise. C) Macroscopic images of EB cells and EBs seeded on scaffolds in EB medium. Cells were fixated and stained for 30 sec. with haematoxylin. All scaffolds had a diameter of $4 \mathrm{~mm}$. Inhomogeneous cell distribution of EB cells and 
EBs, 1 day after static seeding. Low seeding efficiency of EB cells in a spinner flask, after 7 days. Inhomogeneous seeding of EBs in a tube on a roller bank, 1 day after dynamic seeding, and after 7 days of static culture. Note the reduced amounts of cells after the culture period. D) Cells attached per scaffold $(n=6)$ after static and dynamic spinner flask seeding for 1 and 7 days in control medium, starting with 1 (stat 1 and dyn 1) or 5 (stat 5 and dyn 5 ) million cells/scaffold.

All seeding experiments described above were performed in control medium, in the presence of serum proteins. After seeding, the samples were cultured further in chondrogenic medium, in the absence of serum proteins. When the seeded scaffolds were cultured statically in chondrogenic medium for 21 days, the majority of the EB cells or EBs detached from the scaffold. This effect was even more pronounced when cultured dynamically in a spinner flask, where some scaffolds appeared to be completely empty after a 21 day culture period. As a result, little tissue was formed throughout the scaffolds. Nevertheless, in regions of sustained cell attachment, GAGs were expressed by cells and the tissue showed morphology characteristic of cartilage, both in static (Figure 2B) and both dynamic cultures. In conclusion, chondrogenic differentiation of mouse ESCs was achieved on bare scaffolds, but seeding and further culturing methods should be improved to obtain homogeneous tissue formation throughout the scaffolds.

\section{Viability of EB cells and EBs in hydrogels}

A frequently used technique to obtain homogeneous, high cell densities is immobilization in a gel. Furthermore, cells retain a rounded morphology, which might be beneficial for chondrogenic differentiation [188]. Two different gel systems were used in this study. Agarose has been used by many groups as a hydrogel for cartilage tissue engineering or for dynamical loading studies with chondrocytes $[189,190]$. Matrigel is a solubilized basement membrane preparation extracted from the Engelbreth-Holm-Swarm mouse sarcoma, a tumor rich in extracellular matrix proteins.

The viability of EB cells in these two gels was investigated. At various time points, a $1 \mathrm{~mm}$ thin slide was cut from the center of the gel to examine cell viability. In both gels, a homogeneous, high cell density was achieved (Figure 3A). However, we encountered a viability problem. In agarose, the majority of the EB cells died within 2 days and after 7 days hardly any cell survived. The viability of the cells in the center of the Matrigel cylinder gradually decreased, but a superficial layer of viable cells remained, which was still present after 21 days (Figure 3B). It was proposed that cell aggregates display better cell survival than single cells. To investigate this hypothesis, intact EBs were seeded in the two gels and the viability improved considerably. After 21 days, viable EBs were still present in the center of both gels (Figure 3B). However, some EBs showed cell death in their center and some mainly consisted of dead cells (data not shown). 


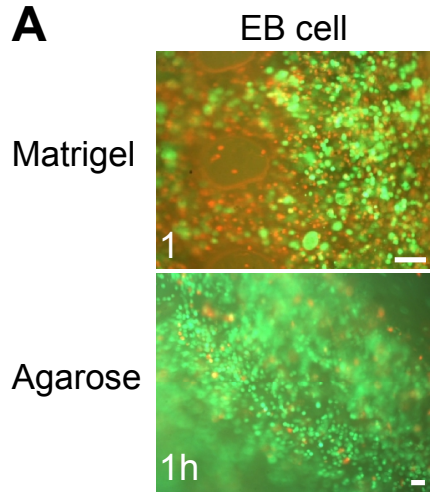

EB cell

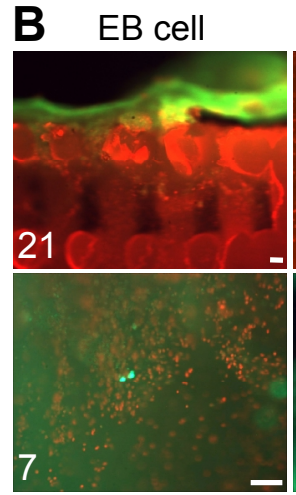

EB cell
EB cell

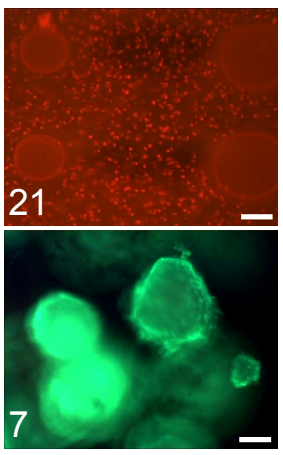

EBs
EBs

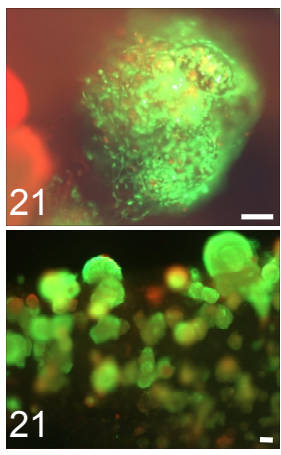

EBs

Figure 3. Viability of single EB cells and embryoid bodies in a gel

A) Viability staining of EB cells after 1 day in Matrigel or 1 hour in agarose indicates homogeneous high cell density of mostly viable cells inside these gels. B) Cell viability staining of single EB cells or intact embryoid bodies (EBs) in either Matrigel or agarose. Cells were cultured for 7 or 21 days, as indicated. Viable cells are indicated by green staining, dead cells by red staining. Note that the scaffold fibers display red background staining. Scale bar: $100 \mu \mathrm{m}$.

\section{Chondrogenic differentiation of EBs in agarose in vitro and in vivo}

After confirming the viability, we investigated whether EBs in agarose could undergo chondrogenic differentiation. EBs were mixed with agarose and then injected into a 3DF scaffold, to improve the mechanical properties of the construct. Since the EBs vary in sizes up to $500 \mu \mathrm{m}$, the 3DF scaffolds were redesigned to allow better infiltration of the EB-gel mixture. The fiber spacing was enlarged from 600 to $1200 \mu \mathrm{m}$ and the double layer architecture increased layer thickness from 150 to 225 $\mu \mathrm{m}$. After 21 days of culture in chondrogenic medium, we observed GAG- and collagen type IIpositive EBs (Figure 4A, diff+), mainly at the periphery of the agarose cylinder. Most of the EBs were fully GAG- and collagen type II-positive, or fully negative. Cell death was seen in the center of some EBs (data not shown). When EBs were cultured in control medium, no cartilage matrix was produced (data not shown).

In a therapeutical application, the cartilaginous tissue will have to survive and possibly develop further in the patient's cartilage defect. Thus, after having established cartilaginous tissue formation in vitro, we now evaluated the capacity of mouse ESCs to form cartilaginous tissue in vivo. For in vivo studies, EBs in agarose on 3DF scaffolds were cultured for 21 days in either incomplete chondrogenic medium (which lacks TGFß3) or control medium and subsequently implanted subcutaneously into the back of 6 immuno-deficient mice. After the in vitro culture period, GAGpositive EBs were present (Figure 4A, diff-), as seen in earlier studies with complete chondrogenic medium (Figure 4A, diff+). After 21 days in vivo, all implants were encapsulated in a teratoma ranging in diameter from 6-25 $\mathrm{mm}$ (Figure 4B). The scaffolds were excised from the surrounding 
tissue and analyzed separately. GAG staining of tissue-sections revealed that no cartilage was formed in the scaffolds. Most of the agarose was still present in the gel-scaffold constructs, as indicated by a distinct border between surrounding tissue and gel with EBs inside (Figure 4B). In the teratomas from both chondrogenic and control implants many tissue types were seen, including cartilage nodules (Figure 4B). Although the in vitro chondrogenic differentiation of EBs in agarose was successful, the experimental setup for the chondrogenic differentiation of mouse ESCs in agarose in vivo was not successful. Cartilaginous tissue was mainly seen in the teratomas surrounding the implants. The EBs inside the agarose did not differentiate further into cartilaginous tissue when implanted in mice.

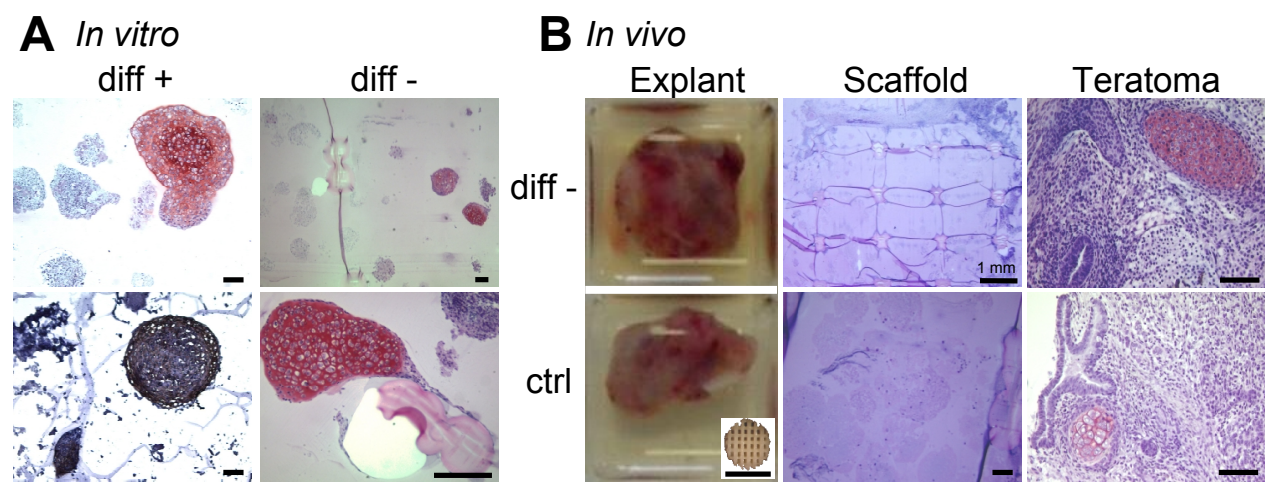

Figure 4. Chondrogenic differentiation of embryoid bodies in agarose in vitro and in vivo

A) Histological sections of embryoid bodies grown in agarose, on a 3DF scaffold and cultured for 21 days in vitro in complete chondrogenic medium (diff+). Cartilage formation is indicated by Safranin-O staining. For in vivo studies, samples were pre-cultured in incomplete chondrogenic medium (diff-). Scale bars represent $100 \mu \mathrm{m}$. B) Left: Macroscopic image of explants after 21 days in vitro in incomplete chondrogenic (diff-) or control (ctrl) medium and 21 days in vivo. The insert shows the scaffold used in this study as size comparison. Scale bar: 0.5 $\mathrm{mm}$. Right: Safranin-O stained sections of the explants. Scaffolds and teratomas were analyzed separately. Scale bar: $100 \mu \mathrm{m}$, unless stated otherwise.

\section{$E B$ cells and EBs in Matrigel in a scaffold in vitro and in vivo}

The in vivo experiments with EBs in agarose did not yield the desired chondrogenic implant. We then investigated the differentiation of mouse ESCs in another gel. Chondrogenic differentiation of both EB cells and intact EBs was studied in Matrigel in a 3DF scaffold. EB cells in Matrigel were cultured for 21 day in chondrogenic medium, which resulted in a superficial tissue layer. This was already indicated by the viability assay, which indicated massive cell death inside the gel (Figure 3B). In this tissue layer, widespread regions of GAG- and collagen type II-positive tissue were present. Some regions even formed a continuous cartilaginous layer (Figure 5A). Culture of EB cells in control medium did not result in chondrogenic differentiation (data not shown). We investigated whether we could also achieve chondrogenic differentiation inside the scaffolds. EBs, which have a higher survival rate than EB cells, were cultured in Matrigel in chondrogenic medium for 21 days. The EBs 
that were cultured in chondrogenic medium expressed GAGs and collagen type II (Figure 5A). The GAGs expressed in EBs stained less intense and appeared to be more diffused than in EB cell cultures of in pellet cultures.

After an in vitro culture period of 21 days, samples were implanted in 6 immuno-deficient mice. The Matrigel-scaffold constructs were retrieved after 21 days and analyzed for GAG expression. A large part of the Matrigel was degraded and replaced by fibrous tissue. The implanted cells continued to differentiate in vivo and various tissue structures were seen, including cartilage nodules. No differences were seen between the EB cell- or EB-containing constructs. The superficial cartilaginous tissue that was present at time of implantation could not be identified anymore. Even though the in vitro results are promising, the in vivo conditions will have to be optimized to obtain a functional tissue-engineered cartilage construct.

A In vitro diff+

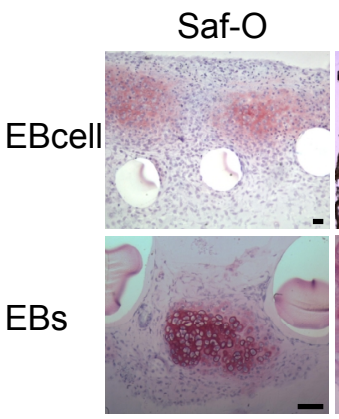

Saf-O

\section{Col2}

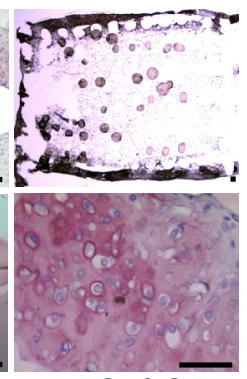

Saf-O

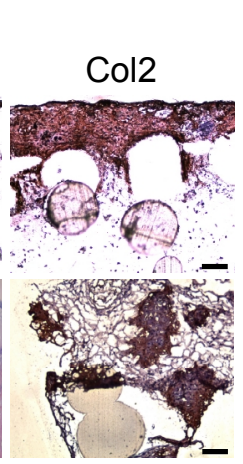

Col2
$\mathbf{B}$ In vivo

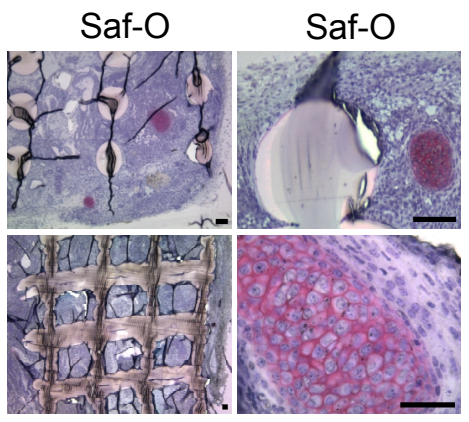

Figure 5. Chondrogenic differentiation of single EB cells or EBs in Matrigel in vitro and in vivo

A) Histological sections of EB cells or embryoid bodies (EBs) combined with Matrigel and a 3DF scaffold, cultured for 21 days in vitro in chondrogenic medium (diff+). GAG formation is indicated by Safranin-O (Saf-O) and collagen type II (Col2) staining. Scale bars represent $100 \mu \mathrm{m}$. B) Safranin-O stained sections of constructs cultured for 21 days in vitro in chondrogenic medium (diff+) and subsequently 21 days in vivo. Scale bar: $100 \mu \mathrm{m}$.

\section{Discussion}

The focus of this study was to assess the application of embryonic stem cells in cartilage tissue engineering. In this first attempt to use ESCs as a cell source, we encountered ESC-specific characteristics that were sometimes beneficial and sometimes disadvantageous to engineer a cartilage graft. We also faced well-known tissue engineering challenges such as choice of differentiation protocol and scaffold, cell seeding efficiency and homogeneity, and use of a less optimal in vivo experimental model system. 
We demonstrated that ESCs undergo chondrogenic differentiation in pellets, on scaffolds and in gels. However, we did not obtain homogeneous cartilaginous tissue. During EB formation the cells differentiate at random, which results in a heterogeneous cell population at the start of chondrogenic differentiation. By omitting the EB formation step, a homogeneous, undifferentiated cell population can be subjected to chondrogenic differentiation. However, little to no cartilaginous tissue was observed in pellet cultures of mouse ESCs instead of EB cells (data not shown). By a selection strategy, a more homogeneously differentiated cell population can be obtained. Nakayama et al. isolated a mesodermal progenitor cell fraction from EBs, by selecting for flk-1 and PDGFR $\alpha$ expression. Single and double positive cell fractions formed more cartilage nodules in pellet cultures than double negative fractions in serum-free chondrogenic medium containing TGFß3, but full cartilage was never observed [101]. The homogeneity of the cell population might be improved by choosing other selection markers, or selecting at a different time point.

The differentiation medium used in this study was optimized for bone marrow-derived mesenchymal stem cells $[97,99]$ and not for ESCs. Human mesenchymal stem cells require TFGß3 for chondrogenesis, and in these pellets intense Safranin-O staining was observed throughout the pellet [99]. In our studies, the chondrogenic effect of TGFß3 was not evident, as indicated by qPCR data from pellet cultures. The EB cell fractions isolated by Nakayama et al. appear to be at different stages in the chondrogenic pathway, since they responded differently to TGFß3. For the flk-1 fraction, TGFß3 is necessary, whereas for the PDGFR $\alpha$ fraction, cartilaginous regions were also formed in the absence of TGFß3 [101]. TGFß3 alone was not sufficient to obtain full cartilage in mouse ESC-derived EB pellet cultures. Nakayama et al. investigated the role of three factors known to be involved in embryonic chondrogenesis: TGFß, PDGF and BMP. PDGF-BB or BMP4 alone did not have a chondrogenic effect, but the addition of PDGF-BB or BMP4 to the chondrogenic medium lead to cartilage ECM deposition throughout the entire pellet. Thus, not only the growth factor combination, but also time of application and the concentration make the choice of differentiation medium complex. In further research, we will use a chondrogenic differentiation medium which is optimized for ESCs in our tissue engineering studies on scaffolds, to achieve more cartilage formation, both in vitro and in vivo.

The culture method during differentiation also plays an important role. As the first step in chondrogenesis during the development of an embryo is the condensation of mesenchymal cells and a 3D tissue is required to repair cartilage defects, we have chosen 3D culture methods. More chondrogenic differentiation was observed in $3 \mathrm{D}$ cultures when compared to $2 \mathrm{D}$ cultures $[98,111]$. 
In 3D hydrogel cultures of intact EBs, we observed fully GAG and collagen type II-positive EBs, despite the intrinsic heterogeneity of EBs. This might imply that randomly differentiated EBs show a degree of differentiation plasticity. Cells in EBs might dedifferentiate and redifferentiate along the chondrogenic pathway, possibly as a result of cell-cell signaling. Based on the fully cartilaginous EBs and the widespread cartilaginous tissue in the dense, superficial layer when the EB cells were combined with Matrigel, we will continue to use our approach of seeding cells at a high density in a 3D environment.

Besides the differentiation protocol, scaffold choice and cell-scaffold interactions are important for the in vivo performance of ESCs in cartilage tissue engineering. We chose a porous biodegradable scaffold, PEOT/PBT, which has been investigated for bone [184,191,192], skin [193-196] and cartilage $[180,181,197]$ tissue engineering. Two architectures, compression moulded and 3D fiber deposited, were investigated. Homogeneous, high efficiency seeding of porous scaffold has been a challenge in other studies. The seeding method, static or dynamic, influences the seeding efficiency and homogeneity. Some studies claim more efficient seeding [198], whereas others found lower seeding efficiency in a spinner flask compared to static seeding [199,200]. More homogeneous [200] or less uniform cell distribution [199] was also observed. Perfusion seeding increased the cell seeding efficiency and uniformity [199]. In our studies, static seeding on CM scaffolds was inefficient and not homogeneous. The pores of the scaffolds should be fully interconnected to allow cells to reach all parts of the scaffold. The 3DF scaffolds meet this requirement better than CM scaffolds [180]. On 3DF scaffolds, both static and dynamic seeding did not result in high seeding efficiency and homogeneous tissue formation. A more critical factor than the seeding method was the affinity of the mouse ESCs for the 300PEOT55PBT45 scaffolds, which appeared to be lower than the bovine and human chondrocytes used in earlier studies $[180,181,185,201-203]$. Especially in spinner flask cultures, the cells easily detached from the scaffolds. The pores of 3DF scaffolds are relatively large and accessible to medium flow when compared to CM scaffolds, which might result in more shear stress on the attached cells, when cultured in a spinner flask. Surface treatment of the 300PEOT55PBT45 improved cell attachment [185], but in serum-free medium the effect is less strong. The serum proteins present in the chondrocyte medium are beneficial for efficient cell attachment [185], but FBS inhibits chondrogenesis in our differentiation protocol (data not shown). Even preincubating the scaffolds and seeding in FBS containing control medium and subsequent culturing in serum-free chondrogenic medium did not result in the desired efficient seeding and tissue formation. 3D fiber deposition is a versatile technique to fabricate well-defined and custommade scaffolds. Pores can be varied in size and shape, whilst maintaining $100 \%$ interconnectivity. In 
the $0^{\circ} / 90^{\circ}$ architecture used in this study, the EB cells and EBs could easily flow through and out of the scaffold. By creating scaffolds with smaller fiber spacing, staggered fiber positioning in successive layers, or changing the fiber orientation using smaller angel steps (e.g. $0^{\circ} / 30^{\circ} / 60^{\circ} / 90^{\circ}$ ), the EB cells and EBs can be caught in the smaller pores. Another method to increase the cell density in the scaffolds is depositing a fine mesh of thin fibers between the larger 3DF fibers by electron spinning technique (Moroni et al., in press). Cells can attach to or aggregate between these fibers, and EBs will be entrapped in the mesh. Furthermore, 3DF scaffolds of other PEOT/PBT compositions or other biodegradable polymers for which the ESCs have a higher affinity can be fabricated. In further research, the scaffolds will be optimized to increase tissue formation on the scaffold.

In this paper, we investigated a different seeding method to improve seeding efficiency. The ESCS could be homogeneously distributed in a gel at high cell densities, and subsequently seeded in a 3DF scaffold. Unfortunately, cell survival within the gels was poor. In both gels, cells were still viable directly after seeding, so the encapsulation itself at less optimal temperatures did not cause immediate cell death. Although the survival of EB cells on the outside of Matrigel is suggestive of nutrient limitation inside the gel, this cannot explain why EB cell do not survive on the outside of agarose, whereas chondrocytes do survive within agarose [188]. Cells can attach to the ECM proteins which are present in Matrigel, suggesting that cell-cell or cell-matrix interactions are required for the survival of EB cells in gels. This might stimulate signaling pathways in the cell, which are beneficial for cell survival. When intact EBs, in which cell-cell contact was ubiquitous, were seeded in the gels, a much better cell survival was achieved throughout the gels. The influence of the gel and matrix components on the survival of ESCs will be investigated in further research. By comparing different hydrogels, and by adding matrix components to the gel, the requirements for cell survival and efficient differentiation will be characterized.

Nutrient availability is another important factor for cell survival. Small aggregates of 20 to 50 ESCS were encapsulated in agarose microcapsules with a diameter of $150 \mu \mathrm{m}$ and continued to proliferate in culture [204]. Encapsulation of mouse ESCs in alginate-PLL microcapsules with a diameter of 500 $\mu \mathrm{m}$ did not reduce the viability of the cells up to 20 days in culture [205]. However, the gels in our studies are larger and the cells might have died of nutrient limitation in the gels. Nutrient diffusion can be improved by diluting the hydrogel. As the mechanical support of our constructs comes from the 3DF scaffold and not from the gel itself, the limitation of gel dilution will merely lie in a possible faster degradation rate of the diluted hydrogel. Nutrient diffusion can also be improved by culturing the gels in a spinner flask or bioreactor. During dynamic culturing, there is better nutrient flow through the constructs, which is beneficial for cell survival. 
After a 21 day in vitro culture period, the EBs in agarose still had their original shape and size, and did not seem to have proliferated. Although agarose itself does not inhibit cell growth [188], it provides substantial resistance to the diffusion of ECM proteins [206]. In Matrigel the cells were able to proliferate, as can be seen in the formation of a dense superficial tissue layer. The GAGs expressed by the EBs were diffused, and the gel itself shows more change from its original shape during culture. As such, the EBs shape and borders are less maintained after a 21 day culture period. Matrigel even stimulated chondrogenesis of chick limb bud mesenchymal stem cells [207]. Thus the properties of the gel can influence cell survival, cell and ECM distribution and differentiation.

When the in vitro differentiated construct were implanted in immuno-deficient mice, the cells continued to proliferate and differentiate in vivo. Some cartilaginous tissue was formed in vivo, but some of the in vitro differentiated tissue was lost upon implantation. For the EB cells seeded in Matrigel, it is possible that the superficial cartilaginous tissue layer was disturbed or destroyed during the insertion of the construct into the subcutaneous pocket. The loss of the cartilaginous EBs in agarose and Matrigel might be a result of cell death or dedifferentiation. It is not likely that all implanted cells died, since we found cartilage nodules after implantation, and these were not likely to result from host fibrous tissue in-growth. The predifferentiation in vitro apparently was not sufficient to maintain a stable chondrogenic phenotype in vivo. The lack of chondrogenic signals in the ectopic implantation site might result in dedifferentiation of the implanted cartilaginous tissue. Cells can be seeded on "instructive" scaffolds, from which chondrogenic signals can be released from, such as the appropriate growth factors. A novel emulsion-coating method for the controlled release of proteins from PEOT/PBT scaffolds has been developed $[208,209]$. As such, chondrogenic differentiation can be stimulated both during in vitro differentiation and after implantation. The loss of cartilage phenotype was also observed in a study where intact chondrogenic micromasses derived from synovial membrane mesenchymal stem cells were implanted subcutaneously in immunodeficient mice [210]. The exact mechanism remains to be elucidated, since adipose tissue-derived mesenchymal stem cells continue to express cartilage ECM proteins [211] and chick limb bud mesenchymal stem cells suspended in Matrigel form hyaline cartilage discs [207] upon subcutaneous implantation in immuno-deficient mice. By explanting the constructs at different time points after implantation, insight in the fate of implanted cells and cartilaginous tissue can be obtained.

In one experiment, implantation in the back of immuno-deficient mice resulted in the formation of teratomas. The ability to form teratocarcinomas in vivo is a well-known characteristic of undifferentiated mouse ESCs. In our studies, we implanted constructs, which had been 
differentiated in vitro for 3 weeks. The formation of a teratoma from differentiated mouse ESCs implies that despite the presence of differentiated cartilaginous tissue, cells capable of forming teratomas still exist in these constructs. This was also observed after injection of 9-15 day old EB cells that were differentiated into the hepatocyte lineage, implying that undifferentiated cells are still present in these cultures [212]. Besides differentiation status of the ESC-derived cells, the high numbers of implanted cells might stimulate teratoma formation [212,213]. The site of injection [212] or implantation also plays a role in the formation of a teratoma. In an experiment by Wakitani et al., the injection of undifferentiated mouse ESCs into the knee joint resulted in less tumor formation than subcutaneous implantation [162]. Thus, the implantation of ESCs in an orthotopic site might also reduce teratoma formation. Ideally, we would like to implant the ESCs in a cartilage defect. The knee joint of an immuno-deficient rat is the largest animal model in which long term immunosuppression is not required. In this model we can investigate the functionality of the implanted cartilaginous tissue and the long term stability of the chondrocyte phenotype of pellets or small scaffolds. However, the main problem is the lack of an appropriate large animal model in which a relevant sized critical defect can be created to implant ESCs on a larger scaffold.

In this paper, we presented results of cartilage tissue engineering with ESCs. Cartilaginous tissue was consistently formed in vitro in the relatively simple chondrogenic differentiation protocol used in this study. High cell amounts could easily be obtained, and homogeneous high cell density seeding was achieved by using a gel seeding method. Viability of encapsulated cells was improved by seeding cell aggregates instead of single cells. Other challenges, such as homogenous differentiation in vitro and in vivo, choice of most optimal scaffold and gel characteristics and stable cartilage formation in vivo, will be explored in further research.

\section{Acknowledgements}

We would like to thank Jan de Wit of Erasmus MC Rotterdam for supply of iB10 mouse ESCs, testing of sera and general discussion. We would also like to thank Sanne Both for assistance with implantation studies. J.M.J was supported by STW grant TPG 5923, JdB was supported by a Senter/Novem grant.

The collagen type II monoclonal antibody (developed by T.F. Linsenmayer) was obtained from the Developmental Studies Hybridoma Bank developed under the auspices of the NICHD and maintained by The University of lowa, Department of Biological Sciences, lowa City, IA 52242, USA. 


\section{CHAPTER 5 EFFICIENCY OF CARTILAGE FORMATION BY MOUSE AND HUMAN EMBRYONIC STEM CELLS}

Jojanneke M. Jukes, Sanne K. Both, Clemens A. van Blitterswijk, Jan de Boer

Institute for Biomedical Technology, Department of Tissue Regeneration, University of Twente, Enschede, The Netherlands

But I still haven't found what I'm looking for I still haven't found what I'm looking for - U2 - The Joshua Tree 


\title{
EFFICIENCY OF CARTILAGE FORMATION BY MOUSE
}

\section{AND HUMAN EMBRYONIC STEM CELLS IN SERUM-FREE \\ CHONDROGENIC MEDIUM}

\begin{abstract}
Embryonic stem cells (ESCs) have been used to study chondrogenesis, and have been investigated as a potential cell source for cartilage tissue engineering. Cartilage formation by adult stem cells has been achieved in pellet cultures in serum-free chondrogenic medium with transforming growth factor-beta (TGFß). In this manuscript, we investigated the efficacy of this protocol for mouse and human ESCs and assessed the necessity of the medium components of the chondrogenic medium. In tissue engineering, cells are combined with scaffolds. Therefore, we also analyzed cartilage formation by mouse and human ESCs in various hydrogels and on polymeric or ceramic scaffolds. We conclude that cartilaginous tissue was formed by mouse and human ESCs, although with varying efficiency and not homogeneously. Whereas one mouse ESC line could be easily triggered to form cartilage, cartilage formation by human ESCs is far less robust. The chondrogenic medium is a good basis for cartilage formation by embryonic and adult stem cells, but it will need improvement to efficiently form a tissue-engineered cartilage implant.
\end{abstract}




\section{Introduction}

The formation of cartilage by embryonic stem cells (ESCs) has been investigated by both fundamental and applied researchers. Whereas developmental biologists try to unravel the factors involved in chondrogenesis in the developing embryo, tissue engineers try to form cartilage from ESCs to repair a defect.

Chondrogenesis has been studied for many years. Early studies used chick or mouse embryos, embryonic limb cultures or mature chondrocytes, and more recent studies included stem cells. Mouse ESCs $[10,11]$ have been proven to be a valuable in vivo model system to study genes involved in chondrogenesis. ESCs are pluripotent, which means they can form all tissues from any of the three germ layers. Mouse ESCs can be injected into a blastocyst, and after implantation in the uterus, ESCS will contribute to the formation of all tissues in the developing chimeric embryo. In ESCs, gene activity can be inactivated or activated through gene knock out or overexpression, respectively, and the effect can be studied in the developing chimeric embryo, or adult chimeric mouse [78]. In addition, adult and embryonic stem cells provide an in vitro model to study factors that are involved in the many steps of chondrogenesis without the necessity to generate transgenic mice.

Culture conditions and growth factors, such as transforming growth factor beta (TGFß), that stimulate the formation of cartilage have been elucidated from chondrogenesis studies. As such, improved knowledge of chondrogenesis in embryonic development will contribute to improved cartilage repair strategies. Cartilage has limited repair capacity. The chondrocytes are embedded in their matrix and cannot migrate to the defect site and stem cells cannot migrate to the defect site in the avascular cartilage. One approach to repair a cartilage defect is tissue engineering, in which chondrogenic cells are combined with a scaffold material, such as polymeric scaffolds or hydrogels. Because of the limited availability of chondrocytes, the potential of stem cells, such as human mesenchymal stem cells (MSCs) or ESCs, for cartilage tissue engineering has been investigated.

First, the formation of cartilage by stem cells had to be established. A decade ago, two groups described the chondrogenic differentiation of multipotent human bone marrow-derived MSCs. They used a pellet culture system, which mimics precartilage condensation, and cells were cultured in serum-free chondrogenic medium containing TGFß $[99,100]$, which was a modified version of medium used for chondrogenic differentiation of rabbit MSCs [97]. This medium and the pellet culture have since then been the basis for many studies investigating the chondrogenic differentiation of stem cells. MSCs from other sources, e.g. adipose tissue [68], umbilical cord [214] or fetal blood, liver and bone marrow [69], and MSCs from other species such as goat $[215,216]$ and 
bovine bone marrow [217] have been successfully differentiated into the chondrogenic linage. Cartilage formation by human MSCs on scaffolds or in gels has also been achieved in chondrogenic medium $[102,218]$.

Mouse and human ESCs [12] have been differentiated into the chondrogenic lineage in vitro as well. First studies used proliferation medium supplemented with chondrogenic growth factors [87] or coculture systems with chondrocytes $[35,91]$ on tissue culture plastic. However, the formation of cartilage tissue was more efficient in a 3D environment such as a micromass or pellet culture [98]. Previously, we have successfully transferred the protocol for MSCs to mouse ESCs. Cartilage nodules were formed by mouse ESCs in pellet cultures, in hydrogels and on scaffolds when cultured in chondrogenic medium $[101,102]$.

Our interest is the formation of a tissue-engineered cartilage implant using ESCs, rather than studying chondrogenesis. Therefore, as a read-out of our differentiation experiments, we decided to focus on the histology of the cartilage tissue, and examined cell morphology and matrix staining for glycosaminoglycans (GAGs). These two factors are present in most histological scoring systems of cartilage implants [219-222].

In this study, we used chondrogenic medium and the pellet culture system as the basis to investigate the efficiency of cartilage formation by mouse and human ESCs.

\section{Materials and methods}

\section{Mouse ESC culture}

Mouse ESC line iB10 was cultured as described previously [102]. In brief, cells were plated at a density of 5000-10000 cells $/ \mathrm{cm}^{2}$ on gelatin-coated tissue culture flasks. Mouse ESCs were cultured in $50 \%$ mouse ESC proliferation medium consisting of Dulbecco's Modified Eagle's Medium (DMEM, Biowhittaker) containing $4.5 \mathrm{mg} / \mathrm{ml} \mathrm{D-glucose,} \mathrm{10 \%} \mathrm{fetal} \mathrm{bovine} \mathrm{serum} \mathrm{(selected} \mathrm{batch} \mathrm{for} \mathrm{mouse}$ ESC culture, Greiner), $0.1 \mathrm{mM}$ non-essential amino acids (NEAA, Sigma), $4 \mathrm{mM}$ L-glutamine (Invitrogen), $100 \mathrm{U} / \mathrm{ml}$ penicillin (Invitrogen), $100 \mu \mathrm{g} / \mathrm{ml}$ streptomycin (Invitrogen) and $50 \%$ of Buffalo rat liver cell-conditioned mouse ESC proliferation medium [176]. Prior to use, $1000 \mathrm{U} / \mathrm{ml}$ Leukemia Inhibitory Factor (Esgro, Chemicon International) and $50 \mu \mathrm{M}$ 2-mercapto-ethanol (Gibco) were added to the medium. Cells were grown at $37^{\circ} \mathrm{C}$ in a humidified $5 \% \mathrm{CO}_{2}$ incubator and passaged with $0.05 \%$ trypsin/EDTA before reaching confluence. 
The iB10 ESC line is a subclone of the E14 line, which has been isolated from 129/ola mice. In addition, we cultured two wild type ESC lines isolated from C57BL/6 mice (C57BI6-1 and C57BI6-2), kindly provided by Prof. van der Horst, Erasmus MC Rotterdam, the Netherlands [223] and a mouse ESC line isolated from C57BL/6 mice expressing a GFP transgene engineered to be expressed specifically in chondrocytes (Col2GFP), kindly provided by Prof. Ung-il Chung, University of Tokyo, Tokyo, Japan [84].

To initiate differentiation, embryoid bodies (EBs) were formed by culturing mouse ESCs at 20,000 cells $/ \mathrm{cm}^{2}$ in non tissue culture-treated 6-well plates (Greiner) in $4 \mathrm{ml} \mathrm{EB}$ medium (DMEM, 10\% FBS, $0.1 \mathrm{mM}$ non-essential amino acids, $4 \mathrm{mM} \mathrm{L-glutamine,} 100 \mathrm{U} / \mathrm{ml}$ penicillin, $100 \mu \mathrm{g} / \mathrm{ml}$ streptomycin and $50 \mu \mathrm{M}$ 2-mercaptoethanol) for 4 days. To obtain a single cell suspension, EBs were dissociated with $0.25 \%$ trypsin/EDTA. EB cells or intact EBs were seeded onto poly(ethylene oxide terephthalate) poly(butylene terephthalate) (PEOT/PBT) scaffolds [182], onto 2-3 $\mathrm{mm}$ porous ceramic particles [224], in $0.5 \%$ agarose (Sigma) in PBS, $0.75 \%$ alginate (Sigma) in PBS, Matrigel (BD Biosciences) or Puramatrix (3DM Inc).

\section{Human ESC culture}

Human ESC line SA002 (CellArtis, Sweden) was established as described by Heins et al. [225]. Human ESC colonies were cultured in gelatin-coated organ well culture dishes (Becton Dickinson) on a feeder layer of mitomycin-C inactivated mouse embryonic fibroblasts (MEFs) in VitroHES ${ }^{\text {TM }}$ medium (Vitrolife AB, Kungsbacka, Sweden) supplemented with $4 \mathrm{ng} / \mathrm{ml}$ human recombinant basic fibroblast growth factor (rh-bFGF, Invitrogen). Medium was refreshed every 2 days. Undifferentiated human ESC colonies were mechanically dissociated into pieces of approximately $0.05 \mathrm{~mm}^{2}$ every 4-5 days with a Stem Cell Cutting Tool (Swemed, Vitrolife) and passaged onto a fresh feeder layer.

To form EBs, colonies were cut into larger pieces of approximately $0.2 \mathrm{~mm}^{2}$ and cultured in a non tissue culture-treated plate in VitroHES medium with or without rh-bFGF and with or without various batches of FBS, or in EB medium as described for mouse ESCs. After 4-8 days, EBs were dissociated using $0.25 \%$ trypsin.

\section{Chondrogenic differentiation}

Differentiation into the chondrogenic lineage was performed in chondrogenic differentiation medium consisting of DMEM high glucose (Gibco), $100 \mathrm{nM}$ dexamethason (Sigma), $100 \mathrm{U} / \mathrm{ml}$ penicillin, $100 \mu \mathrm{g} / \mathrm{ml}$ streptomycin, insulin-transferrin-selenite (ITS+1, Sigma), $0.2 \mathrm{mM}$ ascorbic acid 2-phosphate (ASAP, Sigma), $100 \mu \mathrm{g} / \mathrm{ml}$ sodium pyruvate (Sigma), $40 \mu \mathrm{g} / \mathrm{ml}$ proline (Sigma) and 10 $\mathrm{ng} / \mathrm{ml}$ TGF $\beta 3$ (Biovision) $[97,99]$. For pellet culture, aliquots of $0.5 \times 10^{6}$ cells in $1 \mathrm{ml}$ medium were 
centrifuged at $500 \times \mathrm{g}$ for 2 minutes in $12 \mathrm{ml}$ polypropylene conical tubes. The chondrogenic medium was changed every 3 to 4 days.

\section{Histology}

Safranin-O staining was used to analyze tissue morphology and GAG expression. Constructs were fixed in $0.14 \mathrm{M}$ cacodylate buffer ( $\mathrm{pH}$ 7.2-7.4) containing $0.25 \%$ glutaraldehyde (Merck) and dehydrated using sequential ethanol series. Pellets, polymeric scaffolds or hydrogel samples were embedded in glycol methacrylate (Merck) and cut using a microtome to yield $5 \mu \mathrm{m}$ thick sections. Sections were stained with haematoxylin (Sigma), and fast green (Merck) to visualize cells/cell nuclei (blue staining) and Safranin-O (Sigma), which stained sulphated extracellular GAGs pink. Ceramic scaffolds were embedded in methyl methacrylate (LTI, Bilthoven, The Netherlands) and sections were processed on a histological diamond saw (Leica SP1600; Bensheim, Germany). Sections were etched with an $\mathrm{HCl} /$ ethanol mixture and sequentially stained. Cartilage formation was visualized by $0.04 \%$ thionin (Sigma) in $0.1 \mathrm{M}$ sodium acetate (Merck), which stained cells blue and GAGs pink. Histological sections were analyzed using a light microscope (E600 Nikon).

\section{In vivo studies}

Human EB cells were seeded onto 2-3 mm porous ceramic particles [224] and precultured in EB, chondrogenic or osteogenic medium containing dexamethason described for human MSCs [226] for 21 days. Subsequently, the samples were implanted into subcutaneous pockets in the back of immuno-deficient mice (HsdCpb:NMRI-nu Harlan, n=6) for 4 weeks [102]. Osteogenic samples were also precultured for 1 week and implanted for 6 weeks. Experiments were approved by the local Animal Care and Use committee.

\section{Results}

\section{Cartilage formation by mouse ESCS}

We have been able to successfully transfer the chondrogenic differentiation protocol for rabbit [97] and human MSCs $[99,100]$ to goat bone marrow-derived MSCs. Goat MSCs were cultured for 21 days in serum-free chondrogenic medium containing TGFß, resulting in homogeneous cartilage formation (data not shown). Similarly, EB cells derived from the mouse ESC line iB10 were pelleted and cultured in chondrogenic medium for 21 days. Histological analysis and GAG staining showed that cartilage tissue was formed (Figure 1A), but mostly in nodules rather than homogeneously throughout the pellet, as presented earlier in chapter 4 [102]. The yield and size of chondrogenic 
nodules varied per experiment, with 1 to 15 cartilage nodules per cross section, resulting in approximately $10-60 \%$ of cartilage tissue.

A

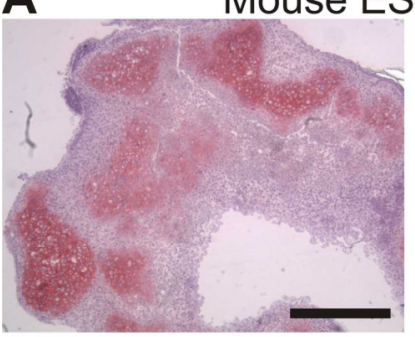

B 21 days

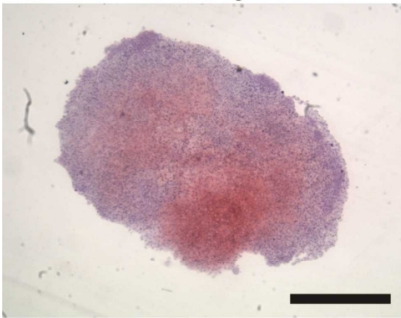

C

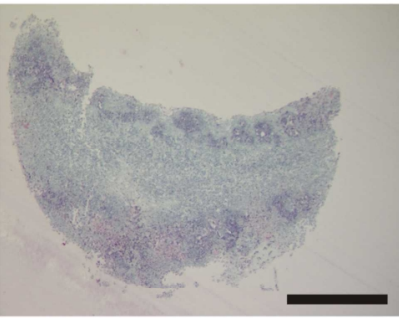

D PEOT/PBT

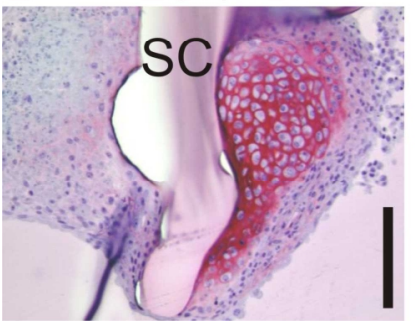

F Agarose

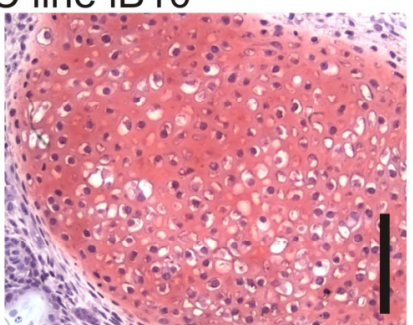

42 days
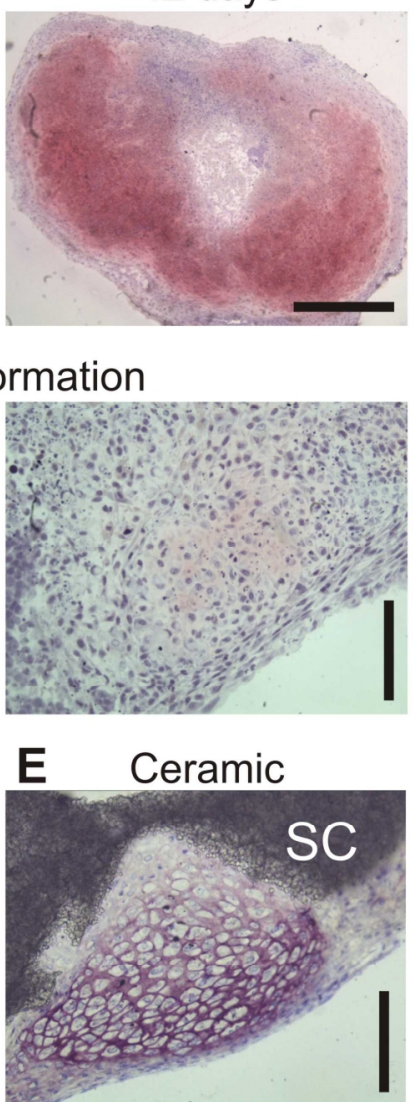

G Alginate

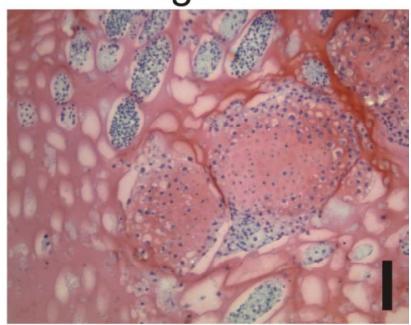

H Matrigel

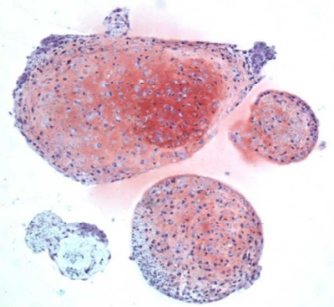

I
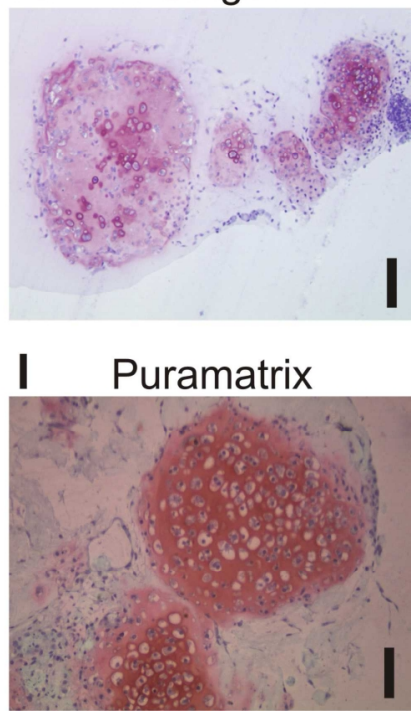

Figure 1. Cartilage formation by mouse ESCs

Representative pictures of histological sections of A) mouse ESC iB10-derived EB cells cultured in pellets in chondrogenic medium for 21 days; higher magnification of a cartilage nodule on the right. B) Pellets cultured for 21 and 42 days in chondrogenic medium. C) No cartilage formation was observed when mouse ESCs were directly cultured in pellets. Cartilage formation by mouse EB cells on D) polymeric PEOT/PBT and E) ceramic scaffolds (sc). Cartilage formation by intact mouse EBs in F) agarose, G) alginate, H) Matrigel and I) Puramatrix. Pink Safranin-O staining of proteoglycans indicates cartilage formation. Horizontal scale bar: $500 \mu \mathrm{m}$, vertical bar: $100 \mu \mathrm{m}$. 
We attempted to increase the homogeneity of cartilage formation. Prolonged chondrogenic differentiation, 42 days instead of 21 days, did not result in more homogeneous cartilage formation (Figure 1B). Because EB formation results in random differentiation of ESCs into cells of all three embryonic germ layers, we tried to direct chondrogenic differentiation at an earlier time point. To this end, we subjected ESCs directly to the chondrogenic differentiation regime. However, when the EB formation step was omitted, no cartilage formation was observed (Figure 1C).

For tissue engineering, the pellet system should be omitted and cells should be seeded and differentiated on scaffolds. Cartilage formation by iB10-derived EB cells was observed on PEOT/PBT [102] (Figure 1D) and ceramic scaffolds [129] (Figure 1E), and when intact EBs were seeded in the hydrogels agarose (Figure 1F) [102], alginate (Figure 1G), Matrigel (Figure 1H) [102] and Puramatrix (Figure 11). We observed differences in the distribution of GAGs in the various hydrogels. In the inert hydrogels agarose and alginate, GAGs seemed to accumulate within the EBs, whereas in the hydrogels which allow attachment of the cells, GAG staining was observed in the EBs as well as in the hydrogel.

\section{Minimal requirements for cartilage formation by mouse ESC line iB10}

We previously observed that goat MSCs can be stimulated into the chondrogenic lineage when exposed to TGFß1 only during the first two days of differentiation [227]. To investigate whether mouse ESCs could also form cartilage with shorter TGFß3 incubation periods, we cultured pellets in the presence of TGFß3 for $0,1,2$ or 3 weeks. We observed that cartilage nodules were formed in all pellets (Figure 2A). In addition, culturing EB cells in the absence of TGFß3 did not result in lower expression of the collagen type II gene [102]. Thus, TGFß does not seem to be a critical factor for chondrogenic differentiation of mouse ESCs.

Based on the observation that TGFß3 can be omitted from chondrogenic medium, we next examined which of the other supplements in the medium were essential for chondrogenic differentiation. Therefore, we cultured pellets of mouse ESC line iB10-derived EB cells in DMEM with antibiotics, supplemented with a single factor, combinations of factors, up to chondrogenic medium. Surprisingly, we observed cartilage formation in all media combinations (Figure 2B), including in DMEM with antibiotics. The efficacy however seemed to be limited and the pellets were very small in medium supplemented with just 1 or 2 factors. Further supplementation resulted in larger and denser pellets and more and larger cartilage nodules (Figure 2B).

From the data above it may seem that cartilage is formed irrespective of the medium used. However, when cells were cultured in EB medium, chondrocyte medium [228] or osteogenic medium [129], we never observed cartilage formation (Figure 2C). Therefore, we examined whether 
fetal bovine serum, which is a supplement in all media mentioned, was inhibiting chondrogenic differentiation. Indeed, when $10 \%$ FBS was added to chondrogenic medium without TGFß3, mouse ESCs would not form cartilage nodules (Figure 2C).
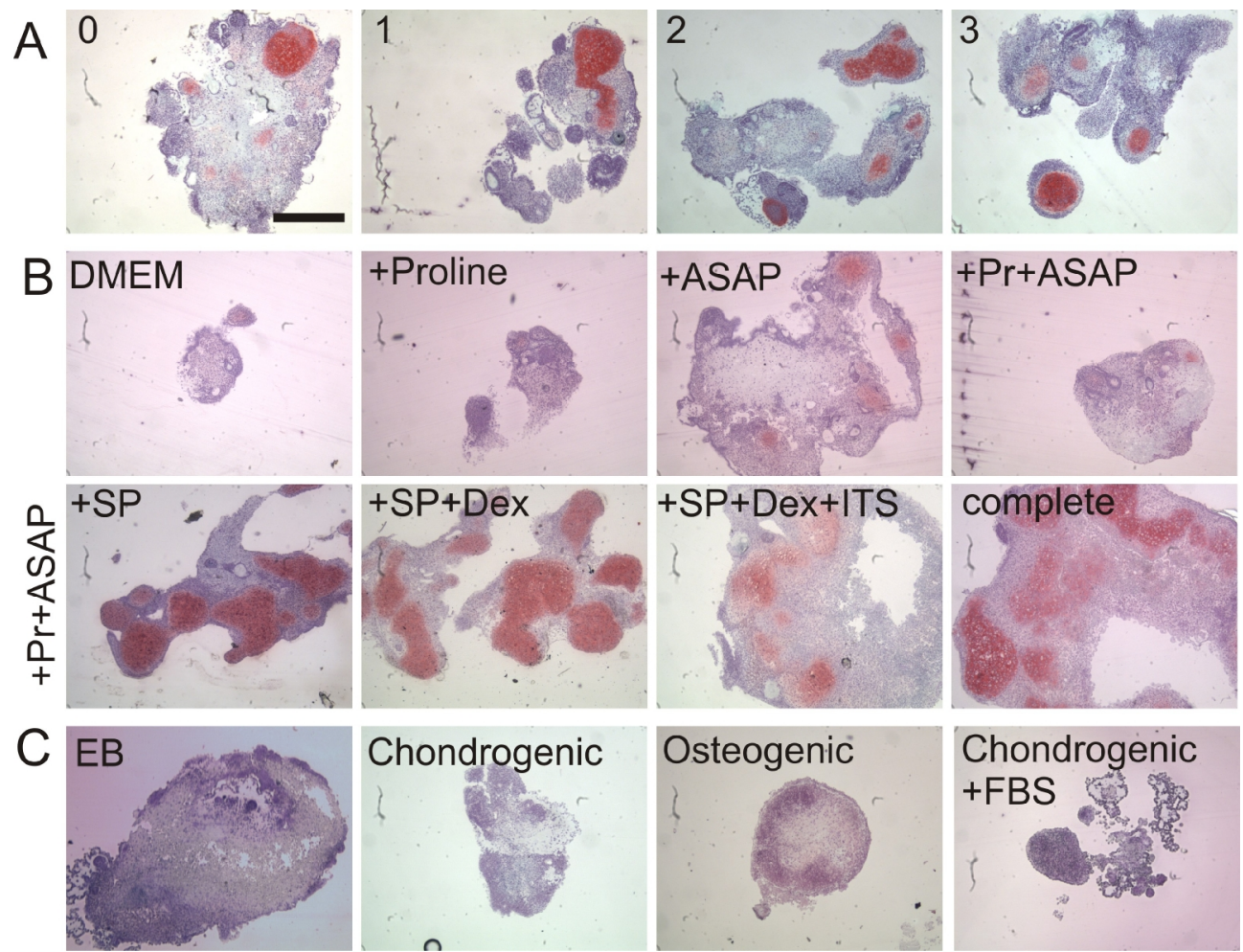

Figure 2. Essential supplements for cartilage formation by mouse ESCs

Safranin-O stained histological sections of mouse ESC-derived EB cells cultured in pellets in A) chondrogenic medium supplemented with TGFß3 for the first 0, 1, 2 and 3 weeks. B) DMEM with antibiotics, supplemented with proline (Pr); ASAP; Pr, ASAP and Sodium Pyruvate (SP); Pr, ASAP, SP and Dex; Pr, ASAP, SP, Dex and ITS; Pr, ASAP, SP, Dex, ITS and TGFß3 (= complete chondrogenic medium). C) EB medium, chondrocytes medium, osteogenic medium or chondrogenic medium with $10 \%$ FBS. Scale bar: $500 \mu \mathrm{m}$, all pictures were taken at same magnification.

\section{Efficiency of cartilage formation by various mouse ESC lines}

Although in principle all ESC lines are pluripotent, differences in proliferation rate and differentiation characteristics between the various cell lines do exist [63]. Therefore, we investigated the chondrogenic potential of 3 other mouse ESC lines. All cells were cultured according to the same protocol and in the same medium used for the $\mathrm{iB} 10$ line, also when the cells were previously cultured under slightly different conditions. First of all, we observed differences in proliferation rates. The population doubling time of $\mathrm{iB} 10$ cells was 15.5 hours, whereas cell lines C57BI6-1, C57BI6-2 and Col2GFP had doubling times of 19.6, 16.8, and 20.9 hours respectively (Figure 3A). 
A

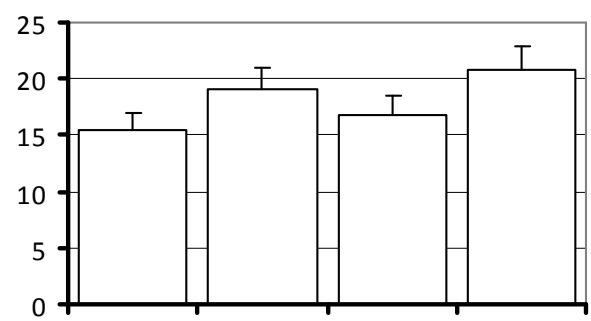

iB10 C57BI6-1 C57BI6-2 Col2GFP

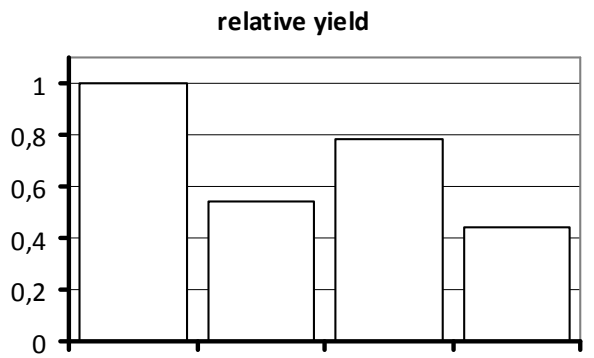

iB10 C57BI6-1 C57BI6-2 Col2GFP

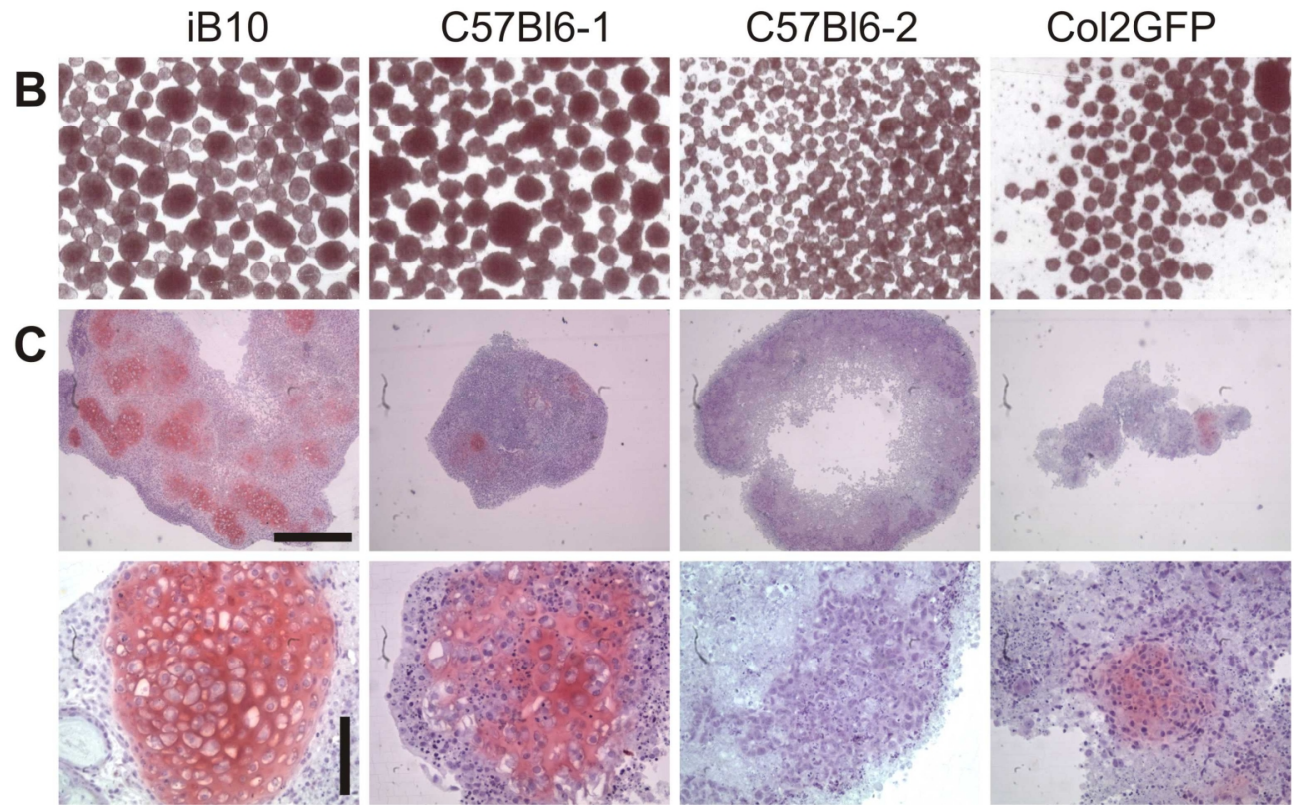

Figure 3. Efficiency of cartilage formation by various mouse ESC lines

A) Population doubling time (h) of 4 mouse ESC lines and the relative yield of cells after 3 days, compared to the iB10 line. B) Efficiency of EB formation after 4 days by 4 ESC lines: iB10, C57BI6-1, C57BI6-2 and Col2GFP. C) Efficiency of cartilage formation by 4 mouse ESC lines, indicated by pink Safranin-O staining of GAGs. Horizontal scale bar: $500 \mu \mathrm{m}$, vertical bar: $100 \mu \mathrm{m}$.

Even more pronounced differences were observed in the efficacy of EB formation. In our standard protocol for iB10 cells, we allow cells to proliferate for 4 days in EB medium because longer EB formation times result in aggregation of individual EBs, without further proliferation (data not shown). The average population doubling time of $\mathrm{iB} 10$ cells during EB formation was 24 hours. This resulted in an average yield of 2.8 million cells after 4 days after initially seeding 175,000 cells in a well. At the same seeding regime, we observed no to one population doubling for the other lines, resulting in smaller and fewer, and in some cases hardly any EBs (Figure 3B). Next, we seeded the cells at a 4 times higher cell density. In this case, 1.5 to 2.5 population doublings were observed in 4 
days, although the EBs were still smaller and fewer in number than the iB10 EBs, especially the Col2GFP EBs.

When pellets were formed from EB cells derived from the 4 ESC lines, some cartilage formation was observed for C57BL6-2 and Col2GFP-derived cells. In this experiment, C57BI6-1 did not show chondrogenic differentiation (Figure 3C).

\section{Cartilage formation by human ESCS}

Next, we analyzed whether the chondrogenic differentiation protocol could be applied to human ESCs (Figure 4A). Because we observed cell death when single human ESCs were used to form EBs, we used larger pieces of manually cut human ESC colonies to initiate EB formation. Interestingly, we observed that the human ESCs remaining in the dish after the colonies were cut for a new passage would grow into rather undifferentiated colonies when the dishes were left in the incubator (Figure 4B). This "regrowth" was used to form a second batch of EBs. We did not observe differences in the efficiency of EB formation or EB morphology between EBs derived from first or second cut of human ESC colonies. Human EBs (Figure 4C) were less regularly shaped than mouse EBs, and formed with much lower efficiency, even when we extended the EB formation time from 4 to 8 days.

To form pellets, EBs were dissociated with trypsin, which resulted in substantial loss of cells during the various washing and centrifugation steps. The yield from 20 dishes with approximately 10 to 12 human ESC colonies was approximately 1.5 million human EB cells. To reduce cell loss, we also prepared pellets using intact EBs. Pellets of 250,000-500,000 cells were cultured for 21 days in chondrogenic medium containing TGFß3. Cartilaginous tissue with cells in lacunae surrounded by positively stained extracellular matrix was formed in some experiments (intact EBs: Figure 4D, EB cells: Figure 4E). In other pellets, we observed faint staining of the ECM, but the tissue did not show typical cartilage morphology (data not shown). The majority of pellets cultured in chondrogenic medium (51 out of 60 pellets analyzed) was negative for chondrogenic differentiation (Figure 4F), but sometimes other tissue structures were observed. We never observed evidence of chondrogenic differentiation, based on morphology or GAG staining, in control pellets cultured in EB medium (data not shown).

In conclusion, we observed some cartilage formation by human ESCs. However, cartilaginous regions were found in less than $10 \%$ of the experiments and remained limited to few and small nodules, with little matrix accumulation. 

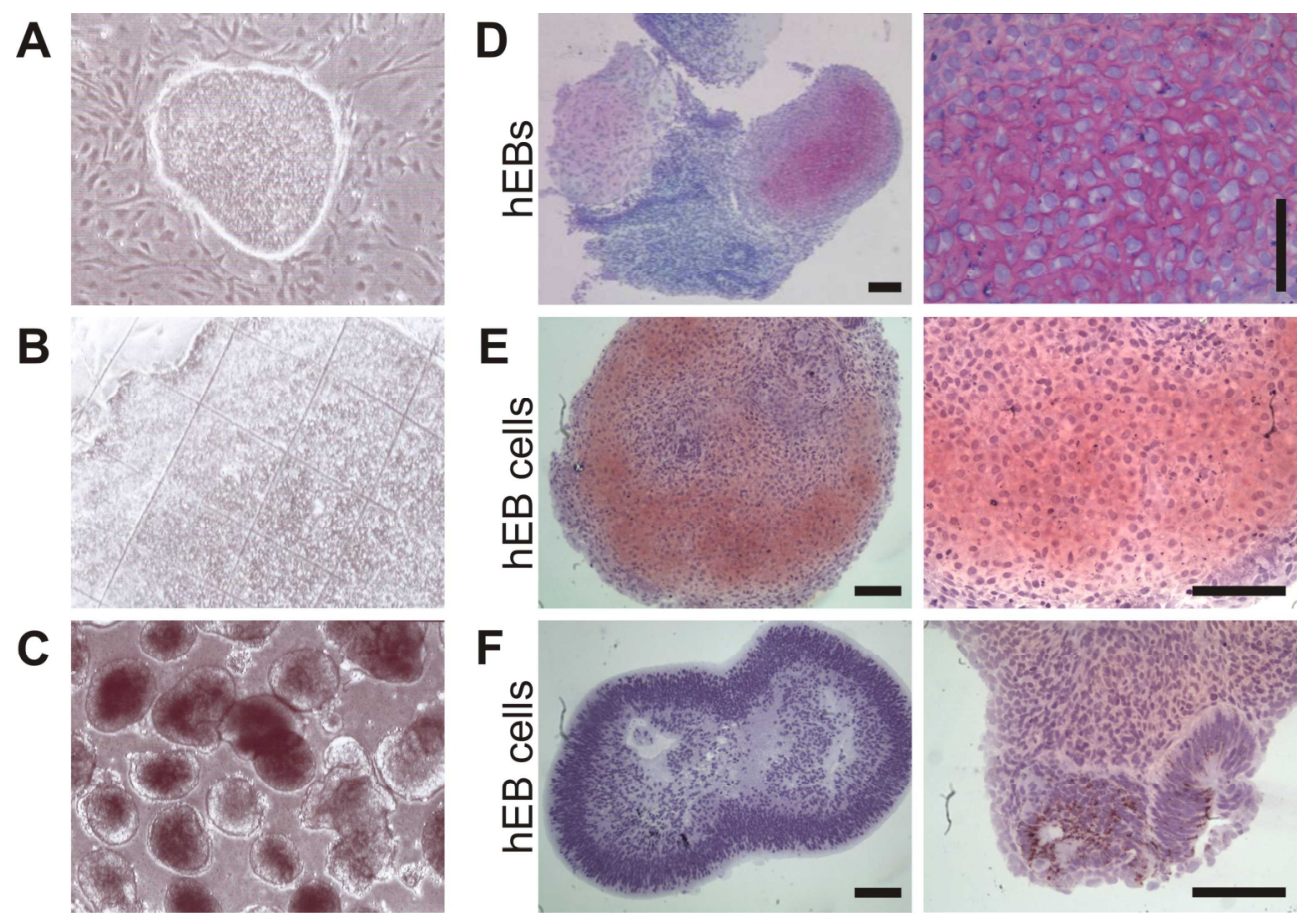

Figure 4. Cartilage formation by human ESCs

A) Undifferentiated human ESC colony on feeder cells. B) Higher magnification image of "regrowth" of undifferentiated human ESCs on cutting pattern after removing the original colony for passaging. C) Human EBs. D-F) Safranin-O stained histological sections of pellets of human EBs D) and human EB cells E-F) cultured in chondrogenic medium for 21 days. Horizontal scale bar: $100 \mu \mathrm{m}$, vertical bar: $50 \mu \mathrm{m}$.

\section{In vivo cartilage formation by human ESCS}

For future clinical applications, 3 dimensional constructs larger than pellets are required. Therefore, we assessed the chondrogenic potential of human ESCs on scaffolds. We used similar approaches as previously applied for mouse ESCs $[102,129]$. We seeded and differentiated human EB cells or intact human EBs in hydrogels (data not shown), on polymeric (data not shown) and ceramic scaffolds (Figure 5A). After 21 days, some cartilaginous regions were observed in the hydrogels (data not shown) or in the pores of the scaffold (shown in Figure 5A for the ceramic scaffold), with a comparable efficiency as described above for differentiation in pellets.

Upon subcutaneous implantation of the cell-seeded scaffolds, large cartilage regions were observed in 4 out of 6 mice when human ESCs were precultured in chondrogenic medium (Figure 5B), but also in 4 out of 6 mice with samples precultured in osteogenic medium (Figure 5C). When cells were cultured in EB medium, only 1 cartilage nodule was observed in the 6 mice (data not shown), compared to 2 to 6 cartilage nodules per samples in the chondrogenic and osteogenic cultures, suggesting that in vitro differentiation did direct the cells into the chondrogenic lineage. The 
cartilage tissue was mainly found in the pores of the scaffolds and aligning the scaffold material, and not as nodules in the surrounding tissue, which is characteristic of cartilage formation in teratomas.
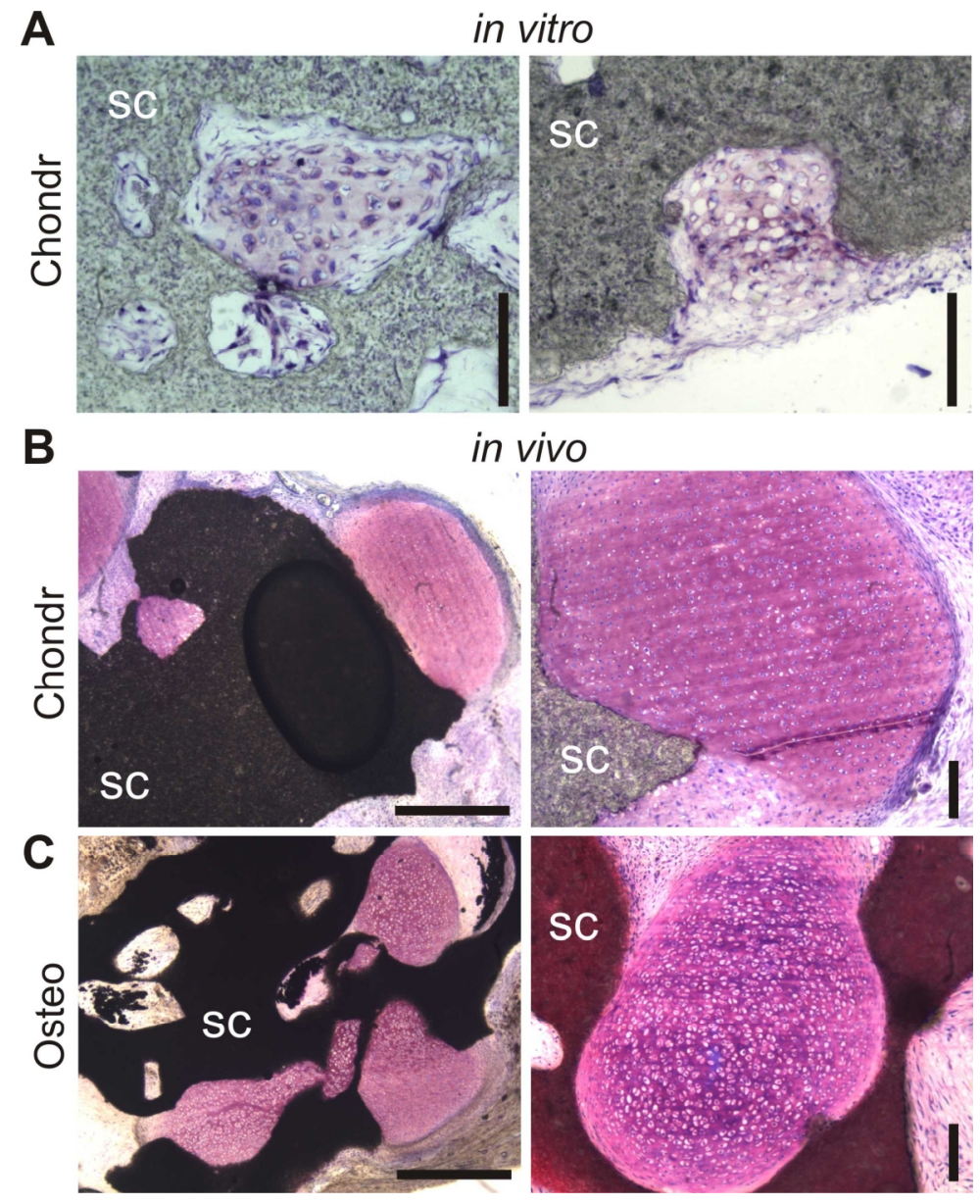

Figure 5. Cartilage formation by human ESCs in vivo

A) Human EB cells seeded on ceramic scaffolds and cultured in chondrogenic medium for 21 days in vitro. B) Human EB cells cultured in chondrogenic medium for 3 weeks and implanted for 4 weeks. C) Human EB cells cultured in osteogenic medium for 7 days and implanted for 6 weeks. Histological sections were stained with thionin which stains cells blue and GAGs pink. Horizontal scale bar: $500 \mu \mathrm{m}$, vertical bar: $100 \mu \mathrm{m}$.

\section{Discussion}

In this study we report cartilage formation by both mouse and human ESCs, although with varying efficiency. We decided to use the most straightforward read out for chondrogenic differentiation, which is the histological analysis of the newly formed cartilage tissue and staining for the presence of glycosaminoglycans in the extracellular matrix. Mouse ESC-derived cartilage tissue could be easily recognized: chondrocytes were embedded in their lacunae and the matrix stained positive for GAGs. 
In a previous study, we further confirmed chondrogenic differentiation of mouse ESCs by analyzing the expression of cartilage-specific markers, such as collagen type II and aggrecan [102]. The morphology of human ESC-derived cartilage was more similar to human MSC-derived cartilage with smaller lacunae and fainter staining of the matrix. We realize that further, more detailed studies concerning the expression of cartilage-specific proteins are necessary to validate our current data.

The chondrogenic medium used in our studies was reported to result in homogeneous cartilage formation by human MSCs [99] in pellet culture. However, we have observed very heterogeneous differentiation by human MSCs from various donors in pellet cultures, varying from abundant to undetectable cartilage formation (data not shown). Similar observations have been reported by many groups. The mechanism is still poorly understood, but donor variation, as also observed for osteogenic differentiation of human MSCs [229], and the heterogeneity of the MSC population are factors known to play a part. Similarly, we observed varying efficiencies of cartilage formation when we analyzed several mouse ESC lines. In this study, we only investigated one human ESC line. More human ESC lines should be investigated to analyze the efficiency of cartilage formation compared to the line we investigated.

Even though many cartilage nodules were formed in the mouse ESC pellets, we never observed homogeneous differentiation. The medium could only drive a subset of the heterogeneous mouse EB cell population into the chondrogenic lineage, and an even smaller fraction of the human EB cells. TGFß3 was not essential for mouse ESCs to differentiate into chondrocytes, whereas more factors will be required for robust cartilage formation by human ESCs. The medium can be a good basis for cartilage formation by stem cells, but optimization is necessary.

The efficiency of cartilage formation by human ESCs was very low. Human ESCs might require longer incubation times than mouse ESCS or human MSCs. Mouse ESCs formed cartilage within 14 days (data not shown), but mouse development in general is much faster than human development. Human MSCs are already more committed, and the time for human ESCs to form this mesenchymallike chondroprogenitor cells might have to be added to the incubation period of 21 days used in this study. In addition, it appears that more committed cells respond better to the administered growth factors than less differentiated cells. No cartilage formation was observed when mouse ESCs were directly submitted to the chondrogenic differentiation regime, whereas 4 day old mouse EB cells did differentiate into chondrocytes. Similarly, when articular chondrocytes were cultured in chondrogenic medium, matrix accumulation was already observed after 7 days, considerably faster than observed for human MSCs [99]. 
We were also interested in the chondrogenic capacity of human ESCS in a tissue engineering context. We seeded cells on ceramic scaffolds and observed some cartilaginous tissue. Besides optimizing the chondrogenic medium, the scaffold material can be designed to enhance cartilage formation. It has been reported that human ESC-derived mesenchymal-like cells did not form cartilage in pellets or simple hydrogels, but did so in functionalized hydrogels [109]. The scaffold material also influences the distribution of the cartilage matrix, as seen in the different hydrogels we analyzed. The material's influence on the efficiency of cartilage formation, on the strength of the construct, on the degradation of the scaffold material and the in vivo stability of the formed cartilage for future clinical applications will have to be further analyzed.

Even though we observed cartilage formation by mouse and human ESCs, it was apparent that the chondrogenic medium used in these studies is not sufficient for efficient cartilage formation by all embryonic and adult stem cells. The variation between mouse ESC and human ESC, and goat and human MSCs, emphasized that animal models cannot simply be transferred to human models. The variation between human MSC and human ESCS clearly pointed out that every cell requires a different set of factors for optimal differentiation into the desired lineage. The variation we observed between donors and cell lines has important consequences for future clinical applications, as each patient or cell line will require optimized medium. It will be important to unravel the critical parameters for cartilage formation, resulting in a general protocol for a large group of patients. 



\section{CHAPTER 6 ENDOCHONDRAL BONE TISSUE}

\section{ENGINEERING USING EMBRYONIC STEM}

\section{CELLS}

Jojanneke M. Jukes ${ }^{1, *}$, Sanne K. Both ${ }^{1,}{ }^{,}$, Anouk Leusink ${ }^{1}$, Lotus M. Th. Sterk ${ }^{2}$, Clemens A. van Blitterswijk ${ }^{1}$, Jan de Boer ${ }^{1}$

\footnotetext{
${ }^{1}$ Institute for Biomedical Technology, Department of Tissue Regeneration, University of Twente, Enschede, The Netherlands

${ }^{2}$ Laboratory of Pathology, Enschede, The Netherlands

*Both authors contributed equally to this work
}

When you've got to feel it in your bones Bones - Radiohead - The Bends 


\title{
ENDOCHONDRAL BONE TISSUE ENGINEERING USING
} EMBRYONIC STEM CELLS

\begin{abstract}
Embryonic stem cells (ESCS) can provide an unlimited supply of pluripotent cells for tissue engineering applications. Bone tissue engineering by directly differentiating ESCs into osteoblasts was unsuccessful so far. Therefore, we investigated an alternative approach, based on the process of endochondral ossification. A cartilage matrix was formed in vitro by mouse ESCs seeded on a scaffold. When these cartilage tissue-engineered constructs (CTECS) were implanted subcutaneously, the cartilage matured, became hypertrophic, calcified and was ultimately replaced by bone tissue in the course of 21 days. Bone aligning hypertrophic cartilage was observed frequently. Using various chondrogenic differentiation periods in vitro, we demonstrated that a cartilage matrix is required for bone formation by ESCs. Chondrogenic differentiation of mesenchymal stem cells and articular chondrocytes showed that a cartilage matrix alone was not sufficient to drive endochondral bone formation. Moreover, when CTECS were implanted orthotopically into critical size cranial defects in rats, efficient bone formation was observed. We report for the first time ESC-based bone tissue engineering under controlled, reproducible conditions. Furthermore, our data indicate that ESCs can also be used as a model system to study endochondral bone formation.
\end{abstract}




\section{Introduction}

Bone tissue engineering is generally approached by combining osteogenic cells with a porous biodegradable ceramic scaffold. Human bone marrow-derived mesenchymal stem cells (MSCs) can be differentiated into the osteogenic lineage by culturing the cells in the presence of the osteogenic differentiation supplements dexamethasone, ascorbic acid and ß-glycerol phosphate [128,226]. Mineralized bone matrix is deposited in vitro as a result of the expression of osteogenic genes [56] and de novo bone formation is observed when human MSCs are implanted into an ectopic or orthotopic site [226]. Even though MSCs from most human donors show osteogenic potential, there is large variation in bone forming capacity by human MSCs, and multipotency is gradually lost upon expansion [229]. Most importantly, bone formation by MSCs is currently insufficient for successful tissue engineering [230]. Besides efforts to increase bone formation by MSCs in vivo, we also explore embryonic stem cells (ESCS) [10-12] as a potential source for bone tissue engineering. ESCs are capable of indefinite, undifferentiated proliferation in vitro and can provide an unlimited supply of cells which can be differentiated into various cell types [36].

Before ESCs can be used in clinical applications, some technical issues have to be addressed, such as the labor-intensive procedure and the use of animal-derived reagents to expand human ESCs, the immunogenicity of allogeneic ESCS and the potential risk of tumorigenicity. Moreover, a differentiation scheme has to be designed to obtain the desired cell or tissue type. Osteogenic differentiation of mouse and human ESCs has been established in vitro, by culturing the cells in medium supplemented with ascorbic acid, B-glycerol phosphate, dexamethasone [127,130,141], BMP2 [136], compactin [136] or vitamin D3 [142]. Mineralization was observed and qPCR analysis showed up-regulation of osteogenic markers such as Cbfa-1/Runx2, osteopontin, bone sialoprotein and osteocalcin. We observed similar results when mouse and human ESCs were differentiated into the osteogenic lineage in vitro (manuscript in preparation). To assess bone tissue engineering using ESCS, we seeded human or mouse ESCs onto ceramic scaffolds and cultured them in osteogenic media for 7 or 21 days. Six weeks after implantation into immuno-deficient mice, no bone tissue was observed in samples of mouse ESCs (manuscript in preparation). For human ESCs, we observed some in vivo mineralized tissue, but no bone tissue, as reported previously [141]. So far, in vivo bone formation by ESCs has only been observed in teratomas. Strikingly, it occurred to us that bone tissue in teratomas frequently aligns hypertrophic cartilage, which resembles the process of endochondral ossification. Most bones in the body are formed via endochondral ossification, which involves the formation of cartilage tissue from condensed mesenchymal cells, and the subsequent replacement of the cartilage template by bone. In contrast, direct conversion of mesenchymal tissue into bone is 
called intramembranous ossification, which occurs primarily in the craniofacial skeleton. In this manuscript, we describe an alternative approach to in vivo bone formation using ESCs, based on the process of endochondral ossification.

\section{Materials and methods}

\section{Cell culture}

Mouse ESC line iB10 was cultured and embryoid bodies were formed as described previously [102]. ESC-derived embryoid body cells were used for differentiation experiments. Human MSCs were isolated from bone marrow aspirates from donors that had given written informed consent [226]. Goat MSCs and calf articular chondrocytes were isolated as described earlier $[46,228]$.

\section{Differentiation}

Aliquots of 1.5 million cells were seeded onto 3 ceramic particles of 2-3 mm, prepared as described by Yuan et al. [224]. For cranial implants, 1 million cells were seeded statically on both sides of the $8 \times 1.5 \mathrm{~mm}$ disc. Further differentiation of stem cells into the chondrogenic lineage was performed in serum-free chondrogenic medium containing TGFß3 [99]. For differentiation of human MSCs, the chondrogenic medium was supplemented with $250 \mathrm{ng} / \mathrm{ml}$ human BMP6 (Biovision) [231]. For differentiation of ESCs into the osteogenic lineage, cells were cultured for another 3 days in medium supplemented with $10^{-7} \mathrm{M}$ retinoic acid (Sigma) and subsequently in medium supplemented with 0.2 $\mathrm{mM}$ ascorbic acid, $2.5 \mu \mathrm{M}$ compactin (Sigma) and $0.01 \mathrm{M} \beta$-glycerol phosphate (Sigma) (adapted from [136]). Osteogenic differentiation of human MSC was described by Both et al. [226].

\section{In vivo studies}

Samples were precultured in chondrogenic or osteogenic medium for 21 days and subsequently implanted into immuno-deficient mice (HsdCpb:NMRI-nu Harlan, $n=6$ ) for 21 days, unless indicated otherwise [102]. For cranial implantation, immuno-deficient rats (Crl:NIH-Foxnrnu, Charles River) were injected subcutaneously with $0.02 \mathrm{mg} / \mathrm{kg}$ buprenorphine (Temgesic) for pain relief. The rats were induced with 4-5\% isoflurane and during the operation they were maintained with a mixture of isoflurane (1.5-3\%), $\mathrm{O}_{2}(200-300 \mathrm{ml} / \mathrm{min})$ and $\mathrm{N}_{2} \mathrm{O}(50-200 \mathrm{ml} / \mathrm{min})$. An incision was made in the skin over the cranium from the middle of the nasal bones to the posterior nuchal line. The periostium was sedated with Lidocaine (2\%) and removed. An $8 \mathrm{~mm}$ trephine dental bur (ACEuropa, Lda) was used to mark the defect site and a $0.7 \mathrm{~mm}$ drill (Synthes) was used to remove the bone to realize the craniotomy. An implant was press fitted into the defect site. Six rats received a sham implant and 7 
rats received a CTEC. The overlaying tissue was sutured back in layers. After 4 weeks, implants were removed and processed histologically as described below. Animals were housed the Central Laboratory Animal Institute (Utrecht University, The Netherlands) and experiments were approved by the local Animal Care and Use committee.

\section{Histological staining and light microscopical analysis}

Samples were fixed in $0.25 \%$ glutaraldehyde (Merck) in $0.14 \mathrm{M}$ cacodylate buffer and dehydrated using sequential ethanol series. Scaffolds were embedded in methyl methacrylate (LTI, Bilthoven, The Netherlands) and sections were processed on a histological diamond saw (Leica SP1600; Bensheim, Germany). Sections were etched with an $\mathrm{HCl}$ /ethanol mixture and sequentially stained to visualize cartilage and bone. Cartilage formation was visualized by $0.04 \%$ thionin (Sigma) in $0.1 \mathrm{M}$ sodium acetate (Merck), which stained cells blue and glycosaminoglycans pink. Bone formation was visualized by $1 \%$ methylene blue (Sigma) and $0.03 \%$ basic fuchsin (Sigma), which stained cells blue and bone pink. Histological sections were analyzed using a light microscope (E600 Nikon). For mineralization studies, ESC-derived EBs or MSCs were grown on tissue culture plates in osteogenic medium for 21 days, fixed and incubated with $5 \%$ silver nitrate (Sigma) under a UV-lamp, until black staining was observed.

\section{Histomorphometry}

Histomorphometry was performed on particles which were explanted at different time points and on the inner circle of cranial implants. Low magnification images were made from 2-3 sections per sample. Scaffold, bone and cartilage were pseudo-coloured and image analysis was performed with KS400 software (Carl Zeiss Vision, Oberkochen, Germany). A custom-made program (University of Utrecht, The Netherlands) was used to measure percentage of cartilage or bone compared to scaffold area.

\section{Statistical analysis}

Statistical calculations were performed with SPSS 14.0 software (Chicago, IL). Histomorphometric data for particles was not normally distributed. Therefore, we used non-parametric tests to compare the amount of cartilage and bone in time (Kruskal-Wallis), and the amount of cartilage and bone at different time points (Wilcoxon signed rank test). For the cranial implants, the samples of the sham group showed little to no bone in-growth in the inner circle whereas bone was formed in all TE samples. Due to the lack of variation in the sham group, we calculated the mean of the three images 
for each sample and a Mann-Whitney $U$ test was used to detect a difference between the two groups.

\section{Results}

\section{Chondrogenic differentiation of mouse ESCs in vitro and bone formation in vivo}

It is well-established that osteogenic human MSC mineralize in vitro (Figure $1 \mathrm{~A}$ ) and form bone in vivo (Figure 1B) [226]. This process of ossification occurs through intramembranous ossification, without the intermediate production of cartilage (data not shown). Moreover, it is known that mouse ESCs, like MSCs, can be induced into the osteogenic lineage in vitro as indicated by mineralization (Figure 1C) and the up-regulation of osteogenic genes. However, in contrast to human MSCs, these osteogenic mouse ESCs did not form bone upon implantation (Figure 1D). Therefore, we assessed an alternative approach: endochondral ossification, in which ESCs first deposit cartilage, which may serve as a template for ossification. In previous studies, we have shown cartilage formation by mouse ESCs in pellets, on polymeric scaffolds and in hydrogels [102]. We now assessed the chondrogenic potential of mouse ESCs on ceramic scaffolds. ESC-derived embryoid body (EB) cells were seeded on ceramic particles and cultured in serum-free chondrogenic differentiation medium containing TGF 33 for 21 days. Regions with typical cartilage morphology, being round cells with lacunae surrounded by extracellular matrix, were found on the outside of the particles and inside the pores (Figure 1E). Cartilage was formed in each sample cultured in chondrogenic medium and about a tenth to a third of the cells on the particles differentiated into chondrocytes. Collagen type II expression was substantially up-regulated compared to control cultures (data not shown). Particles cultured in control or osteogenic medium did not show formation of cartilage (data not shown).

After creating a cartilage template on ceramic particles by differentiating mouse ESCs into the chondrogenic lineage for 21 days in vitro (from now on referred to as cartilage tissue-engineered constructs, CTECS), the next step was to demonstrate in vivo bone formation. Therefore, CTECs were implanted subcutaneously in the back of immuno-deficient mice for 21 days. Bone-like tissue was formed in all samples which were differentiated into the chondrogenic lineage (Figure 1F), in contrast to the samples which were differentiated into the osteogenic lineage in vitro (Figure 1D), in which bone tissue was never observed. The newly formed bone, also known as osteoid, was aligned with osteoblasts, and osteocytes were visible in the mature and mineralized bone tissue (Figure 1G). Bone was formed both on the outside of the particles, as within the pores, and the tissue consisted 
of lamellar bone as demonstrated by polarized light (Figure $1 \mathrm{H}$ ). For the first time, we demonstrate directed, reproducible bone formation using mouse ESCs in vivo.
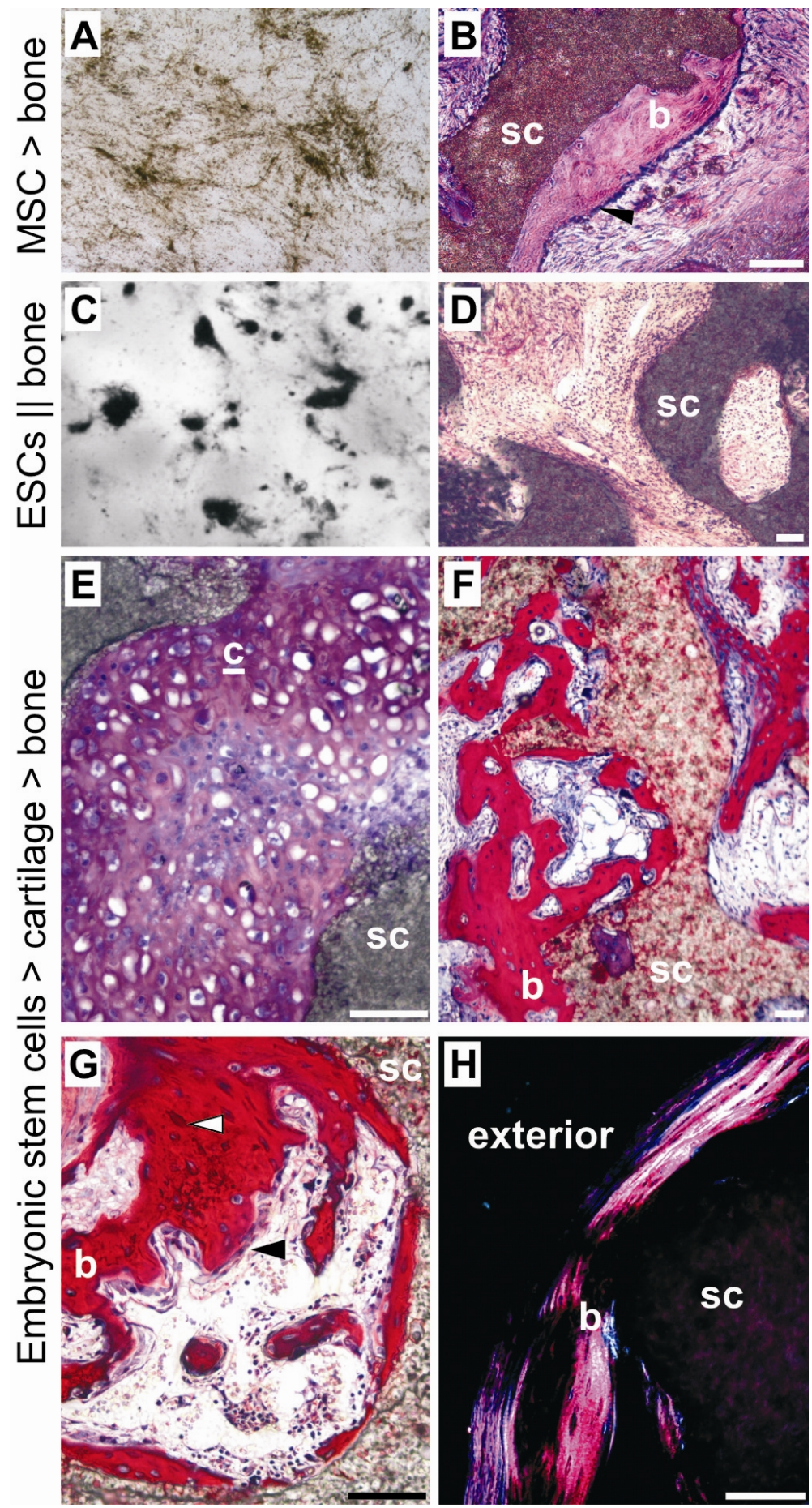


\section{Figure 1. Two approaches for in vivo bone formation by MSCs and ESCs}

A) In vitro osteogenic differentiation of human MSCs cultured on tissue culture plastic for 21 days, indicated by von Kossa staining, which stains mineralized matrix black. B) In vivo, bone is formed by human MSCs, as shown by the methylene blue and basic fuchsin stained sections of cells grown on ceramic particles for 7 days and implanted subcutaneously into immuno-deficient mice for 6 weeks. Bone tissue stains pink and bone-lining cells are indicated by a black arrow head. C) In vitro osteogenic differentiation of mouse ESCs cultured on tissue culture plastic for 21 days, indicated by black von Kossa staining of the mineralized matrix. D) After 21 days, no bone is formed in vivo by mouse ESCs, which were precultured in vitro for 21 days on ceramic particles in osteogenic medium. E) In the process of endochondral ossification, bone is formed on a cartilage template. Mouse ESCs were cultured in chondrogenic medium on ceramic particles for 21 days. Cells displayed a chondrocyte phenotype as indicated by round cells in lacunae surrounded by extracellular matrix, which stained positive for glycosaminoglycans (indicated by pink thionin staining). F) CTECs were implanted subcutaneously for another 21 days to demonstrate bone formation. Bone tissue is stained dark pink by basic fuchsin. G) Higher magnification of bone tissue observed on implanted CTECs. Bone-lining cells are indicated by a black arrow head and osteocytes by an open arrowhead. H) The bone tissue that was formed consisted of lamellar bone as indicated by polarized light. Ceramic scaffolds are indicated by sc, bone by b and cartilage by c. Scale bar represents $100 \mu \mathrm{m}$.

\section{A time course of endochondral bone formation by mouse ESCS}

Next, we investigated the fate of in vitro formed cartilage after implantation and the process of bone formation in vivo. Therefore, we analyzed the CTECS 2, 7, 14 or 21 days after implantation (Figure 2A). At the time of implantation, cartilage tissue was present on the CTECs and hardly any cellular stroma was observed. After 2 days in vivo, cartilaginous tissue was still present on the implanted CTECS, indicating the survival of the implanted tissue. The CTECs showed different amounts of fibrous immature scar-like tissue that showed resemblance to mesenchymal cells in tissue cultures. After 7 days in vivo, the cartilage showed the beginning of maturation indicated by larger lacunae with smaller uniform nuclei. The onset of endochondral calcification was indicated by slight basic fuchsin staining within the mature cartilage. Furthermore, the fibrous stroma became more cellular and dense and contained more vessels. Bone formation was first seen after 14 days in vivo. Bone surrounded hypertrophic chondrocytes and the mineralized cartilage matrix (Figure 2B). In some regions, cartilage seemed to be totally replaced by bone and in other regions, mature cartilage was still present. After 21 days in vivo, hardly any cartilage remained, and more bone tissue was observed than after 14 days. We even observed tissue resembling bone marrow in some bone lacunae (Figure 2A).

The gradual decrease in the amount of cartilage and a gradual increase in the amount of bone tissue in time were confirmed by histomorphometric analysis. After 21 days, there was significantly less cartilage and significantly more bone per scaffold area than at earlier time points. There was also significantly more bone than cartilage after 21 days (Figure 2C). Thus, in the course of 21 days in vivo, almost all cartilage matured and was replaced by bone tissue. 


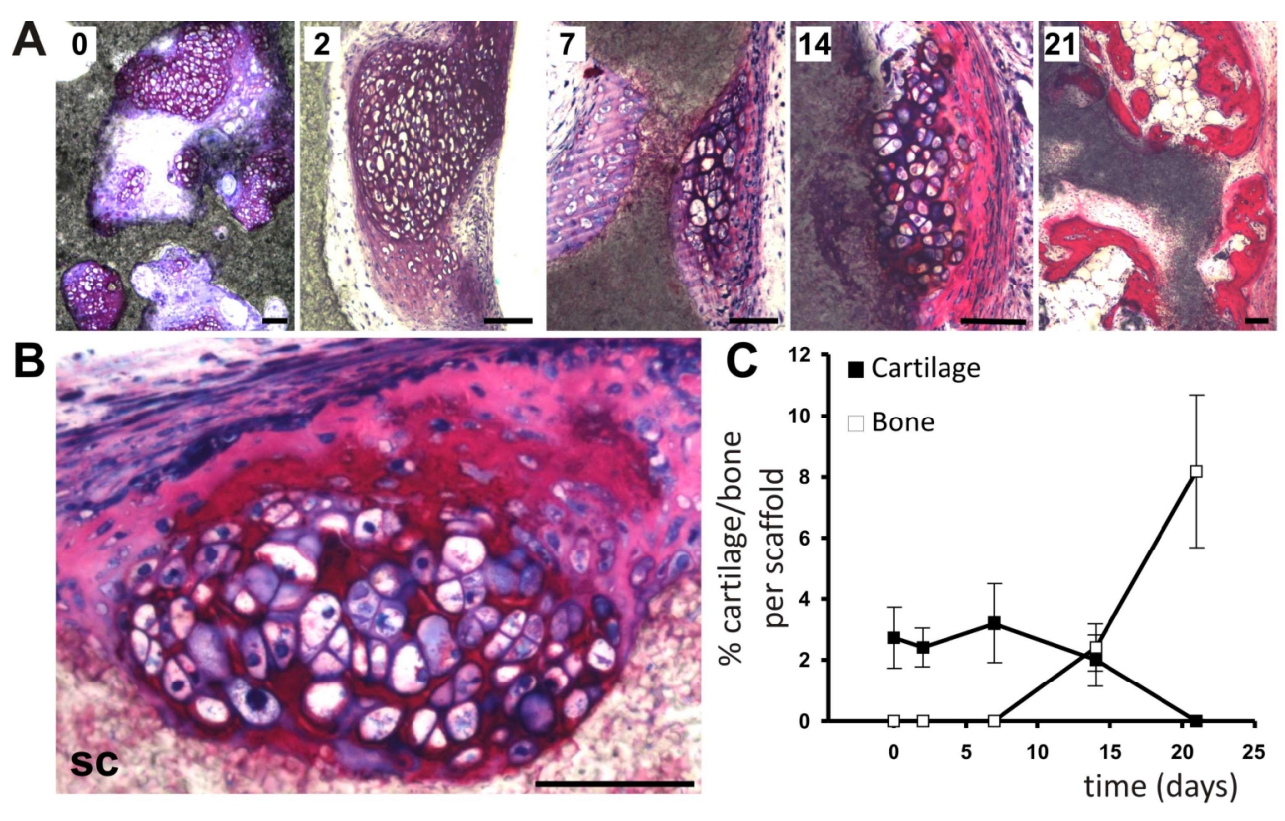

Figure 2. In vivo bone formation throughout time

A) Representative images of histological sections of CTECs 0, 2, 7, 14 and 21 days after implantation. At 0 and 2 days in vivo, cartilage matrix is visualized by pink thionin staining of glycosaminoglycans. At 7, 14 and 21 days in vivo, bone tissue is stained by methylene blue and basic fuchsin staining, which stains cells blue and bone tissue dark pink. Cartilage can still be recognized by morphology, being an extracellular matrix in which single cells in a lacuna can be distinguished. B) Higher magnification image of a CTEC after 14 days in vivo, showing the process of endochondral ossification. Hypertrophic chondrocytes in mineralized cartilage were surrounded by bone tissue. Scale bars represent $100 \mu \mathrm{m}$. C) Histomorphometric analysis of the amount of cartilage and bone per available scaffold area in time.

\section{A cartilage template from ESCs is necessary for endochondral bone formation}

We investigated whether either chondrogenic stimulation or cartilaginous tissue was required for in vivo bone formation. Therefore, we differentiated cells in vitro for 3, 7, 14 and 21 days, and subsequently implanted these samples for another 21 days into immuno-deficient mice. In vitro chondrogenic differentiation for 3 and 7 days did not result in tissue with typical cartilage morphology. After 14 days, the first, mainly small, cartilaginous regions were observed, and more and larger regions of cartilage tissue were formed after 21 days of in vitro culture (Figure 3A). After 14 days in vitro, we scored 0-5 cartilage nodules, with an average of 2.4 (12 sections) and for the 21day CTECs, we observed 11-34 nodules, with an average of 21.7 nodules/section (Figure 3C). After subsequent implantation for 21 days, we scored the amount of bone nodules aligning the ceramic particle in all histological sections (5-7 sections per sample, 6 mice per time point). No bone nodules were observed in the 3 days samples. For the samples that had been differentiated in vitro for 7 days, we observed 1 bone nodule in a few sections, with an average of 0.2 bone nodules/section. 
A In vitro B In vivo
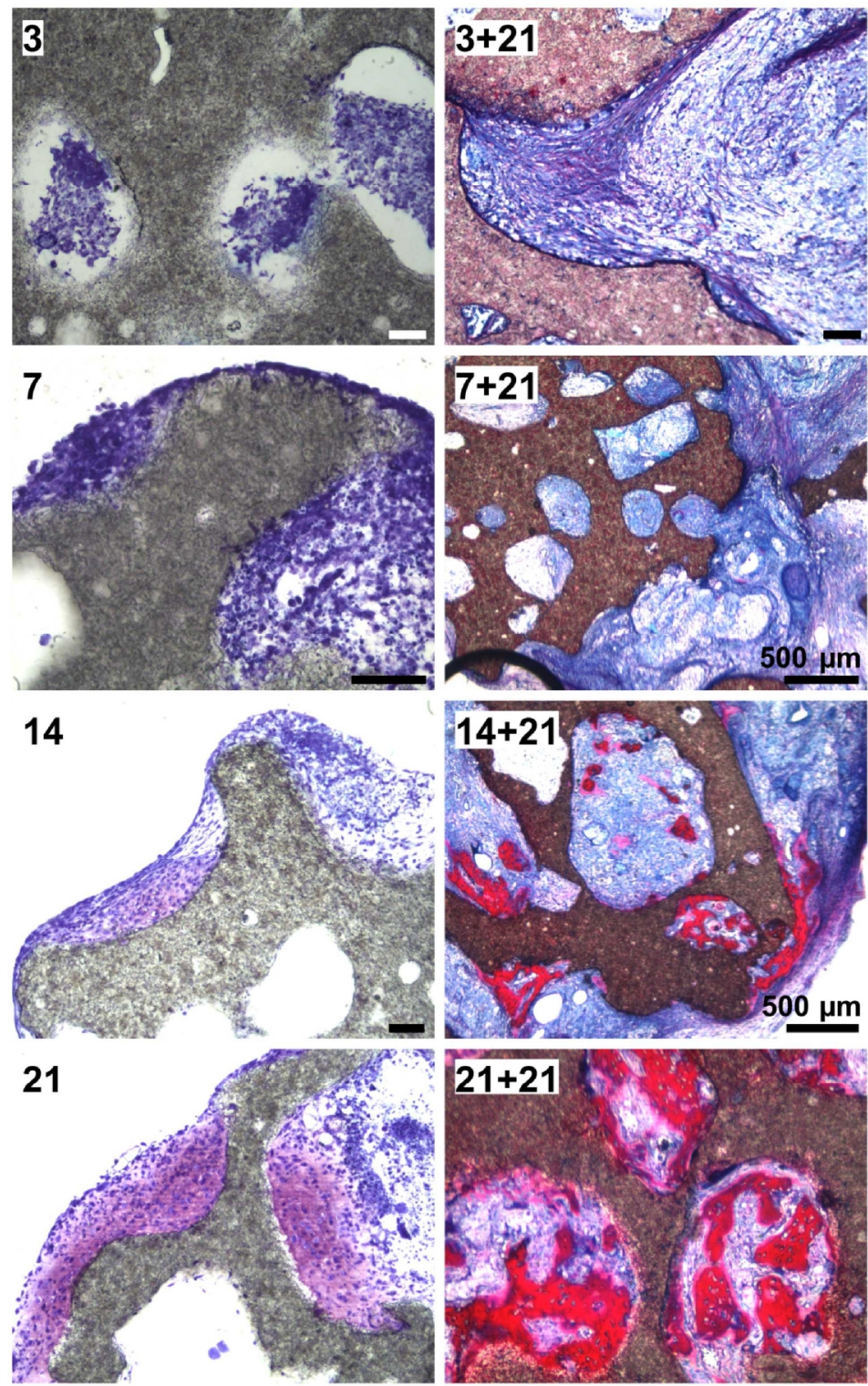

C

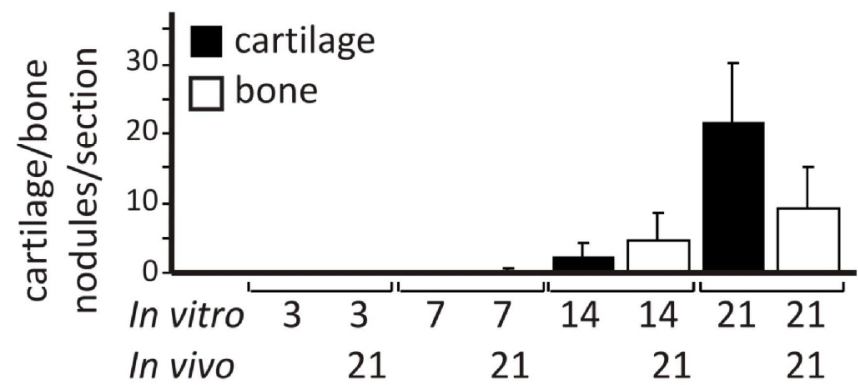




\section{Figure 3. The necessity of a cartilage template for in vivo bone formation using mouse ESCs}

A) Chondrogenic differentiation of mouse ESCs for 3, 7, 14 and 21 days in vitro, as indicated by thionin staining. Cartilage matrix was first observed after 14 days of chondrogenic differentiation and the amount increased in time as seen after 21 days. B) Subsequent implantation for 21 days after in vitro differentiation for 3, 7, 14 and 21 days. Bone tissue was observed in the $14+21$ and $21+21$ days samples as indicated by basic fuchsin staining. Scale bars represent $100 \mu \mathrm{m}$. C) Average amount of cartilage nodules in vitro and bone nodules in vivo in time, scored per section.

For the 14 days-CTECs, we observed 0-13 bone nodules in the sections, with an average of 4.6, and for the 21 day CTECs, we observed 2-20 bone nodules, with an average of 9.2 bone nodules/section (Figure 3C). Thus, bone was mainly observed in the samples that had been differentiated into the chondrogenic lineage for 14 days and 21 days (Figure 3B). In consistency with the higher amount of cartilage in vitro, the highest amount of bone was found in the 21 days samples. We conclude that a cartilage template is required for bone formation.

\section{A cartilage template is not sufficient for endochondral bone formation}

To investigate whether any cartilage template will mature, calcify and will be replaced by bone, we implanted cartilage derived from articular chondrocytes and adult stem cells.

Freshly isolated calf chondrocytes were cultured on ceramic particles in chondrocyte proliferation medium. After 21 days in vitro, cartilage and some fibrous tissue was formed on the ceramic particles (Figure 4A). Subsequently, these constructs were implanted into immuno-deficient mice. The cartilage phenotype was stable in vivo and more cartilage matrix was deposited (Figure 4B). Hypertrophy and calcification of the cartilage matrix was not observed. No signs of endochondral ossification were observed when articular chondrocyte-derived cartilage was implanted.

We also investigated the fate of a cartilage template derived from adult stem cells. Given that even non-stimulated MSCs can form bone in vivo, we had to look for signs of endochondral ossification other than sheer bone formation, such as hypertrophic chondrocytes, calcified matrix and regions where bone aligns and replaces cartilage. We seeded goat and human MSCs on ceramic particles and differentiated these cells into the chondrogenic lineage as described for ESCs. Cartilaginous tissue was formed by goat MSCs, which was almost homogeneous in some samples (Figure 4A). Human MSCs proliferated on the ceramic particles and a small amount of GAG-positive tissue was observed (Figure 4A), limited to one to three small regions per sample.

Next, these CTECS were implanted into immuno-deficient mice for an additional 21 days. Cartilaginous tissue was still observed and bone was formed in vivo (Figure 4B).

Bone formation in human MSC samples remained limited to an average of 2.6 small bone nodules per sample, similar to the rather inefficient chondrogenic differentiation in vitro. No signs of endochondral ossification were observed in these few bone nodules. 


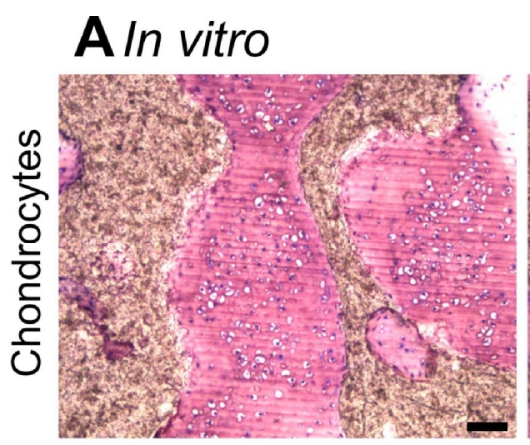

\section{$\mathrm{B}$ In vivo}
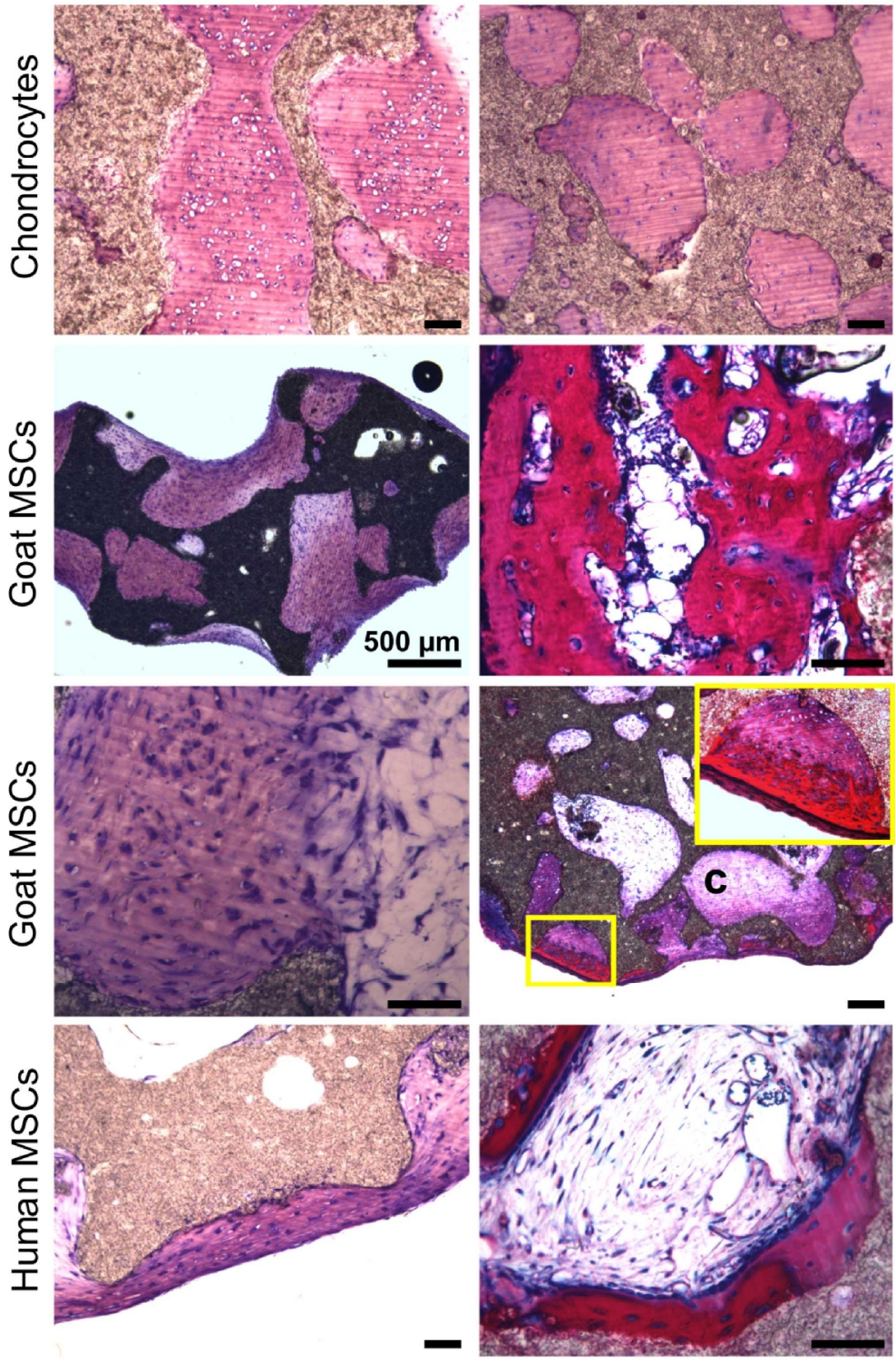

Figure 4. A cartilage template is not sufficient to induce endochondral bone formation

A) In vitro cartilage formation by bovine chondrocytes, goat MSCs and human MSCS, stained by thionin. B) Subsequent implantation of the in vitro samples as displayed in A). No bone was formed in the bovine cartilage samples. Bone was observed in both goat MSCs and human MSC samples as indicated by basic fuchsin staining. In some regions, bone aligned cartilage (inset). Cartilaginous tissue (c) was still present in goat MSC samples. Scale bars represent $100 \mu \mathrm{m}$. 
Extensive bone formation was observed in the implanted goat MSC CTECs: up to 40 bone nodules per section. Most bone appeared to be formed by the process of intramembranous ossification, as also observed when non-stimulated cells were implanted. However, in $14 \%$ of the bone regions, bone aligned cartilage or calcified areas were observed in the cartilaginous tissue. Even though we did not observe the typical replacement of hypertrophic cartilage by bone, as observed with mouse ESCs (Figure 2B), these areas are indications of the onset of endochondral bone formation by goat MSCs. In studies with mouse ESCs, hardly any cartilage was observed after 21 days in vivo (Figure 2C). However, large regions of cartilage were still present in the implanted goat MSCs samples (Figure 4B). Of the implanted large cartilaginous regions, approximately $80 \%$ did not show signs of endochondral ossification after implantation.

Based on these experiments, we conclude that a cartilaginous matrix does not automatically lead to endochondral bone formation, but rather that stem cell-derived cartilage, and especially ESCderived cartilage has a tendency to mature and enter the process of endochondral ossification.

\section{Bone tissue engineering in an orthotopic defect}

The above-mentioned in vivo studies were restricted to ectopic implantation sites. To study bone formation in an orthotopic defect, we implanted CTECs or empty scaffolds in an $8 \mathrm{~mm}$ critical size cranial defect in an immuno-deficient rat. After 21 days, cartilage was observed in all in vitro discs (CTECs, data not shown).

The cranial defect proved to be critical, as bone in-growth did not bridge the sham implant. To distinguish between bone in-growth from the cranium, and bone formation using ESCs, all samples were divided in an outer ring, containing the regions of bone in-growth, and an inner ring, in which bone in-growth was not observed in the sham implants (Figure 5A). The percentage of bone in the inner circle of sham implants and CTECS (Figure 5C) was determined by histomorphometry (Figure 5B). Significantly more bone was observed in the inner circle of CTECS, as compared to sham implants. Hereby, we show efficient bone formation using ESCs in an orthotopic defect. 

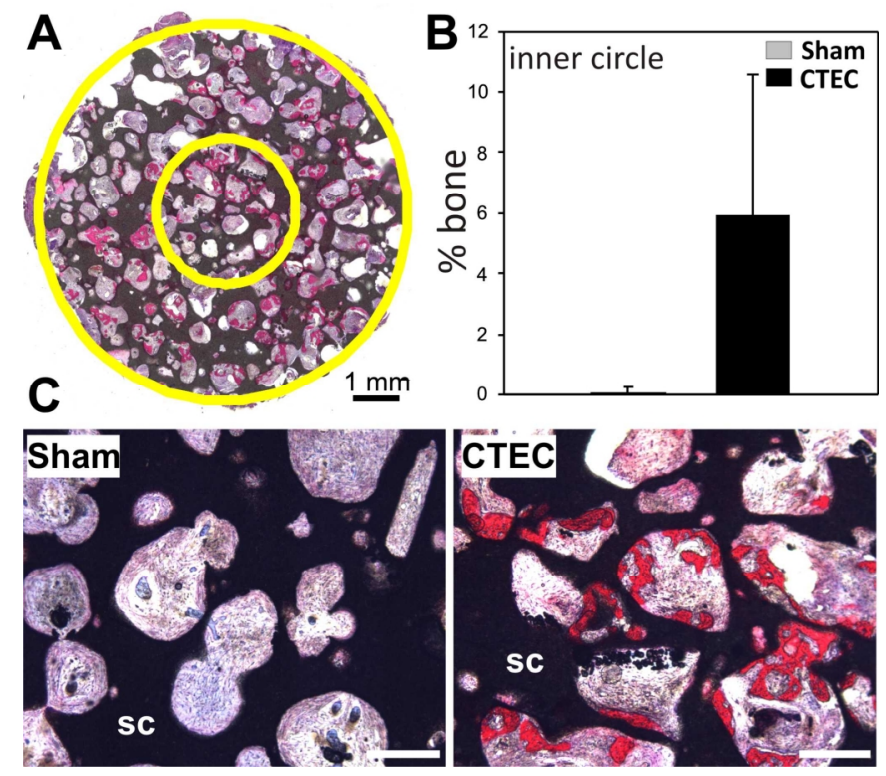

Figure 5. Orthotopic bone formation using ESCs in a rat cranial defect

A) Bone formation in an $8 \mathrm{~mm}$ CTEC implanted for 21 days in a critical size cranial defect in a rat, visualized by methylene blue and basic fuchsin staining. For subsequent analysis, the sample was divided in an outer and inner ring, indicated by yellow circles. B) Histomorphometric analysis of bone tissue formed in the inner circle of sham implants and CTECs. C) Higher magnification view of the inner circle $(2.7 \mathrm{~mm})$ of a sham implant and a CTEC. Scale bar represents $500 \mu \mathrm{m}$, scaffold is indicated with sc.

\section{Discussion}

In this paper, we describe in vivo bone formation using mouse ESCs. We first attempted to directly differentiate ESCs into the osteogenic lineage, based on differentiation protocols and media established for adult stem cells [56] and osteoblasts. Although in vitro results were satisfying, in vivo experiments did not result in bone formation, by either mouse or human ESCs. We opted for an alternative approach by first forming a cartilage template and subsequently allowing the cartilage to be replaced by bone. Using this approach, we demonstrated for the first time directed and reproducible in vivo bone formation using ESCs in ectopic and orthotopic sites. The process is very robust, as we observed bone in all experiments where an ESC-derived cartilage template was formed in vitro. The amount of bone formed in these experiments was comparable to the amounts formed by rat and goat MSCs (data not shown). We also observed endochondral bone formation on polymeric scaffolds, indicating that in vivo bone formation was not induced by and is not exclusive to ceramic scaffolds (data not shown).

Most, if not all, differentiation protocols result in a heterogeneous population of ESCs differentiated into various lineages, but enriched for the desired cell type. Cartilage and bone were formed in all 
experiments, but ESCs also differentiated into other less or more advanced tissue types, like squamous and cylindrical epithelial cells lining cyst-like spaces and tubules, endothelial cells lining (blood) vessels, fat and stroma. In some experiments we even observed teratoma formation. Cartilage, hypertrophic and calcified cartilage and some bone were observed in the teratomas, but in contrast to our directed differentiation, these tissues did not align the scaffold material. For future application, the constructs should be purified from residual undifferentiated ESCs to avoid teratoma formation in vivo. In addition, purification could result in more homogeneous cartilage formation, which might result in improved bone formation.

Currently we are investigating which cell population is responsible for bone deposition. A subpopulation of mesodermal or osteoprogenitor cells might be present in the implanted heterogeneous ESC population, which differentiate into osteoblasts and deposit bone matrix. Alternatively, as during bone growth, blood vessels infiltrate the cartilage matrix and host cells might form bone tissue stimulated by factors secreted by the hypertrophic chondrocytes. Blood vessels close to the cartilage matrix were observed frequently in the samples.

The next step will be to show in vivo bone formation using human ESCs. When human ESCs were differentiated into the osteogenic lineage, small fragments of mineralized tissue were observed in vivo [141], but no osteocytes and osteoblasts were observed. The first step in endochondral bone formation by human ESCs would be the formation of a cartilage template. The chondrogenic potential of human ESCs has been demonstrated in indirect co-culture experiments with primary chondrocytes [35]. Whereas mouse ESCs show consistent cartilage formation, we did not observe cartilage formation when the chondrogenic protocols were transferred to human ESCs (data not shown). Serum-free chondrogenic medium containing TGFß3 did not only result in heterogeneous cartilage formation by mouse ESCs, but the cartilaginous tissue that was formed by human MSCs in our studies was also not homogeneous (data not shown), even when supplemented with BMP6. Further optimization of the growth-factor regime for both adult and embryonic stem cells is necessary $[103,104]$.

Mouse ESC-derived cartilage displayed maturation, calcification and subsequent replacement by bone tissue. Indications of maturation were also observed for human ESC-derived cartilaginous tissue $[35,103]$ Apparently, ESCs followed the route of embryonic development. This implicates that ESCs can be used as a model system to study endochondral bone formation. Where most current models use chicken eggs, in vitro limb cultures, or transgenic mice, our results show that endochondral bone formation can now be studied with ESCs. Transgenic ESCs, rather than transgenic mice can be used to investigate the influence of several genes in the process of endochondral bone formation. Besides in vitro assays, in vivo bone formation can be studied in 
ectopic and orthotopic models. The orthotopic defect used in our studies might not be the most logical model for endochondral bone formation, as it is well-known that the bones of the craniofacium form through intramembranous ossification. However, this study does demonstrate that that the implanted mouse ESCs results in bone formation in an orthotopic defect in a rat.

In conclusion, our data show that mouse ESCs readily undergo endochondral ossification after deposition of a cartilage matrix, which can benefit both bone tissue engineering and the genetic dissemination of endochondral bone formation.

\section{Acknowledgements}

We would like to thank Jan de Wit from Erasmus MC Rotterdam for supply of iB10 mouse ESCs, Hugo Fernandes from the University of Twente and the GDL in Utrecht for assistance with rat cranial defect surgery and Pamela Habibović from the University of Twente for assistance with statistical analysis. JMJ was supported by STW grant TPG 5923, JdB and SB were supported by a Senter/Novem grant. 


\section{CHAPTER 7 POTENTIAL OF EMBRYONIC}

\section{STEM CELLS FOR IN VIVO BONE}

\section{REGENERATION}

Jojanneke M. Jukes, Sanne K. Both, Clemens A. van Blitterswijk, Jan de Boer

Institute for Biomedical Technology, Department of Tissue Regeneration, University of Twente, Enschede, The Netherlands

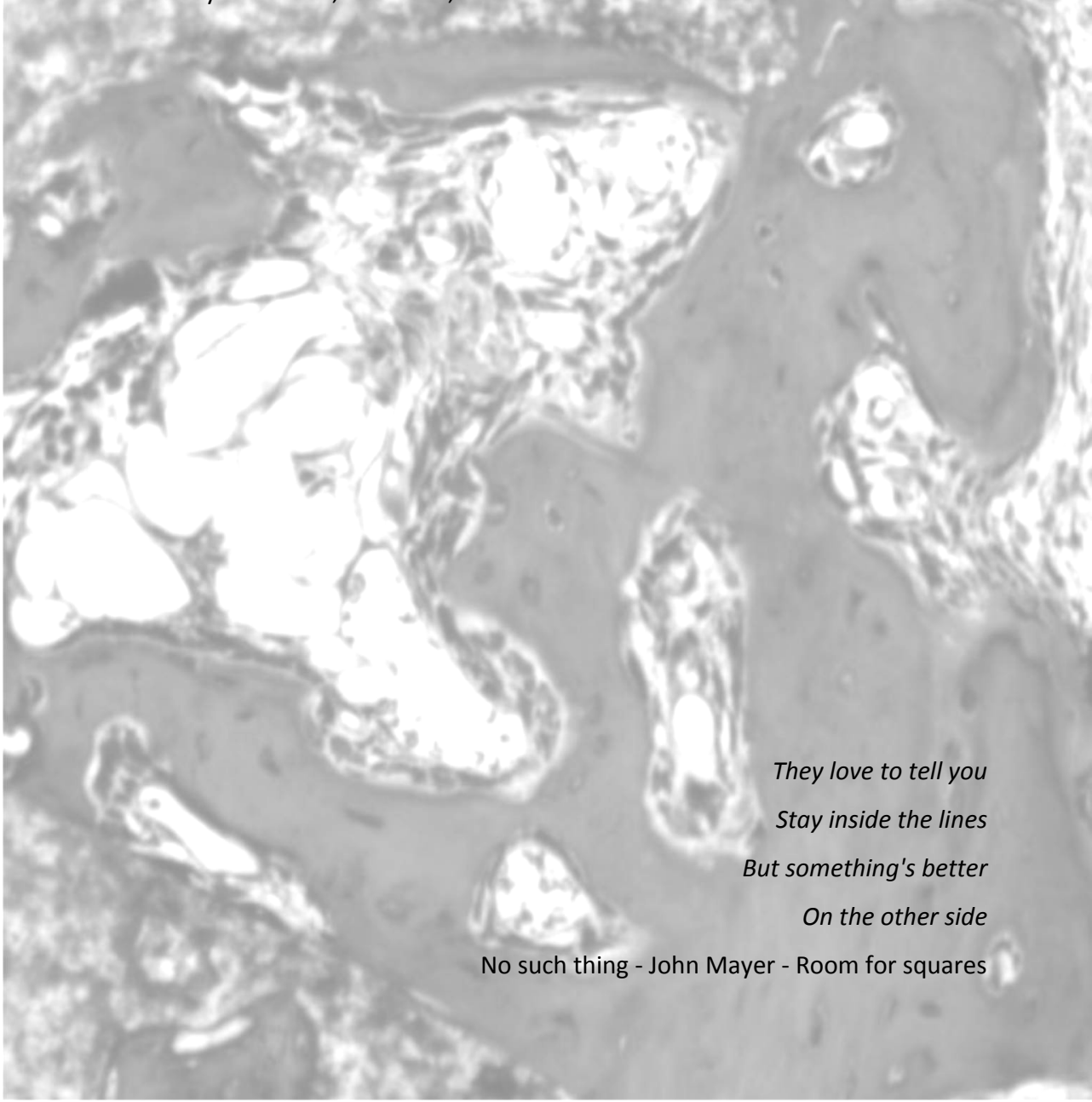




\section{EDITORIAL: POTENTIAL OF EMBRYONIC STEM CELLS}

\section{FOR IN VIVO BONE REGENERATION}

When your little niece falls out of a tree and breaks her arm, she will cry, but the doctor applies a pretty pink cast, and within a couple of weeks, the bone will heal. Unfortunately, when a patient suffers extensive bone trauma, for example after an accident or removal of a bone tumor, the selfhealing capacity of bone is not sufficient to fill the bone defect and surgical intervention is required. The current golden standard for bone therapy is the use of autografts. Healthy bone is harvested from, for example, the patient's pelvis or rib, and implanted into the bone defect. Autologous bone has all the characteristics required for in vivo bone regeneration: osteoconductivity (bone cells migrate over its surface into the graft), osteoinductivity (factors in the graft induce bone formation of uncommitted stem cells), osteogeneity (bone forming cells are present in the graft), absence of immune rejection and load-bearing properties. However, using the patient's own bone does have some drawbacks. There is limited availability of bone in the patient's body, two operations are needed to harvest and implant the bone, and severe chronic pain at the donor site is often experienced by the patient.

When autologous bone cannot be used, there are alternatives, but they do not perform as well as the autograft. Allografts, bone harvested form human cadavers, are available in larger quantities. To reduce the risk of disease transfer, the graft has to be devitalized, but this treatment also reduces osteoinductivity and osteogeneity.

Alternatively, allografts can be processed into an osteoinductive product: demineralized bone matrix (DBM). During preparation, bone minerals and cells are removed, but the osteoinductive proteins retain their activity. The best known osteoinductive proteins are the bone morphogenetic proteins (BMPs). Recombinant human-BMP2 is an FDA approved product and is used for spinal fusion, in which the spine is stabilized by fusing two or more vertebrae. The production and purification of BMPs make this a costly treatment. Another approach is to combine bone forming cells with a support material and to implant this construct in the bone defect. This approach is known as bone tissue engineering. As scaffold material, porous ceramics, used as bone fillers in small defects, are typically chosen. The materials degrade in vivo, leaving newly formed bone in the defect site. Bone forming cells, osteoblasts, cannot simply be isolated from the patient. Bone would have to be harvested, and cells would have to be isolated out of their bone matrix. Stem cells with osteogenic potential are a more logical cell type. Multipotent mesenchymal stem cells (MSCs) can be 
differentiated into, amongst others, bone, cartilage and fat. MSCs have been isolated from several sources, including bone marrow and fat derived from liposuction procedures.

MSCs can be isolated from the patient, making them an ideal candidate to apply in in vivo bone tissue engineering. The great potential of MSCs has been proven by rat and goat MSCs, which form large amounts of bone upon implantation. Unfortunately, so far, human MSCs do not meet the expectations. When cells were implanted into animal models, or applied in clinical trials, the amount of bone formed was insufficient to bridge a large bone defect [230]. Furthermore, donor variation in the amount of deposited bone is observed, which makes it even harder to design the most optimal protocol [229]. The protocols for human MSCs in bone regeneration have to be improved, before they can be used in the clinic. Therefore, scientists have continued to search for cells with the potential to form more bone in a defect. Recently, various groups have studied the osteogenic potential of embryonic stem cells (ESCs). Initial studies applied osteogenic protocols for human MSCs to mouse and human ESCs. In vitro results were very promising, because osteogenic markers were expressed by the cells and mineralization was observed. Next, ESCs were seeded onto ceramic scaffolds, differentiated into the osteogenic lineage in vitro, and implanted into immuno-deficient mice. Unexpectedly, no bone tissue was formed [141]. Although some small regions of mineralized tissue were observed, cells that histologically define bone, i.e. bone lining cells, osteocytes and bone marrow, could not be identified.

Recently, we followed another approach, by mimicking bone formation in an embryo. Rather than directly forming bone, called intramembranous ossification, a cartilage template is first formed, on which bone can be deposited, a process called endochondral ossification. Chondrogenic differentiation of mouse ESCs was induced using protocols developed for cartilage formation by human MSCs. ESCs were seeded onto ceramic scaffolds and differentiated in vitro which resulted in the formation of cartilage. These constructs were subsequently implanted into immuno-deficient mice. In vivo, bone was formed on the cartilage template. In time we could follow the process, from chondrocyte maturation towards hypertrophy, via mineralized cartilage matrix, to the formation of lamellar bone in which osteoblasts, osteocytes, bone lining cells, and some bone marrow-like tissue could be identified. Thus by not following the conventional approach of intramembranous ossification, we were able to form bone using mouse ESCs [129]. We followed the same approach for human ESCs, but under the current conditions, their chondrogenic potential is insufficient to form cartilage in vitro and bone in vivo. 
In theory, ESCs are a good candidate for bone tissue engineering, because they can provide an infinite amount of cells which can form bone. But are they a good candidate in practice, and can we already apply human ESCs in the clinic? The current answer would be not yet or not at all. There are still many limitations concerning using human ESCs. Some drawbacks are ESC-specific. The current proliferation protocols are too complicated and time-consuming to allow scaling up of the cultures to clinically relevant amounts (tens of millions of cells). Their propensity to form teratomas, benign tumors, upon implantation is reason for concern. We observed teratoma formation in mouse ESC samples that had already been differentiated in vitro for 3 weeks. Apparently, a small residue of undifferentiated cells remains, which can result in devastating tissue growth in vivo. Another characteristic of ESCs is their pluripotency, which means they can form every cell type of the human body. This is an advantage, but at the same time a disadvantage. So far, we only observed heterogeneous cartilage tissue formation, and it will be a challenge to form homogeneous tissue using such a pluripotent cell population. Protocols will have to stimulate differentiation into the desired tissue while simultaneously inhibiting differentiation into another lineage, or include cell sorting. Another drawback is that unlike MSCs, ESCs cannot be isolated from the patient. To avoid immune rejection upon implantation, ESC banks would have to be established to match the patient's cells. Recently, the generation of induced pluripotent stem cells (iPS cells) has provided the possibility for a source of autologous pluripotent stem cells $[160,161]$. However, iPS cells will also hold the ESC's disadvantages of pluripotency and tumorigenicity.

Do these disadvantages mean we should stop investigating the potential of ESC for in vivo bone regeneration? Again, the answer will be not yet or not at all.

Isolated only a decade ago, the work with human ESCs is still in its infancy. Progress has been made, and impressive results have been obtained. However, the limitations described above are a huge challenge for researchers and we expect it will take many years before the use of human ESCs in bone repair will be safe and efficient enough. Perhaps the translation of ESCs into the clinic will turn out to be too complicated, or too expensive.

However, there might an alternative use for human ESCs in bone repair. An important question in the endochondral bone formation approach described above for mouse ESCs is which cells are responsible for the actual bone formation. Do the implanted mouse ESCs, which had not differentiated into chondrocytes, form bone, or are host MSCs attracted by signals released from the cartilage matrix? In the latter case, the cartilage matrix could be considered osteoinductive, and this would put the use of ESCs into a new perspective. Many of the above-mentioned disadvantages of 
using ESCs in the clinic could be avoided by not implanting the living cells. By allowing ESCs to create a cartilage matrix in vitro and subsequently processing the tissue, we might be able to create an osteoinductive decellularized cartilage matrix (DCM). As such, human ESCs can be used to produce an off the shelf product to repair bone defects. We might even use other cell sources to create this DCM. Mouse ESCS could be an equally good candidate as human ESC and we know that MSCs can also form cartilage. We observed some endochondral bone formation when we implanted cartilage derived from goat MSCs. The most ideal cell source for DCM production would be a cell line that is easy to maintain and differentiate. More experiments are required to establish the ideal conditions for endochondral ossification using various cell sources. However, by keeping an open mind, and using less conventional approaches, we might surprise ourselves again, and find a new golden standard for bone repair therapies by either using ESCs, or products produced by ESCs. 



\section{CHAPTER 8 GENERAL DISCUSSION}

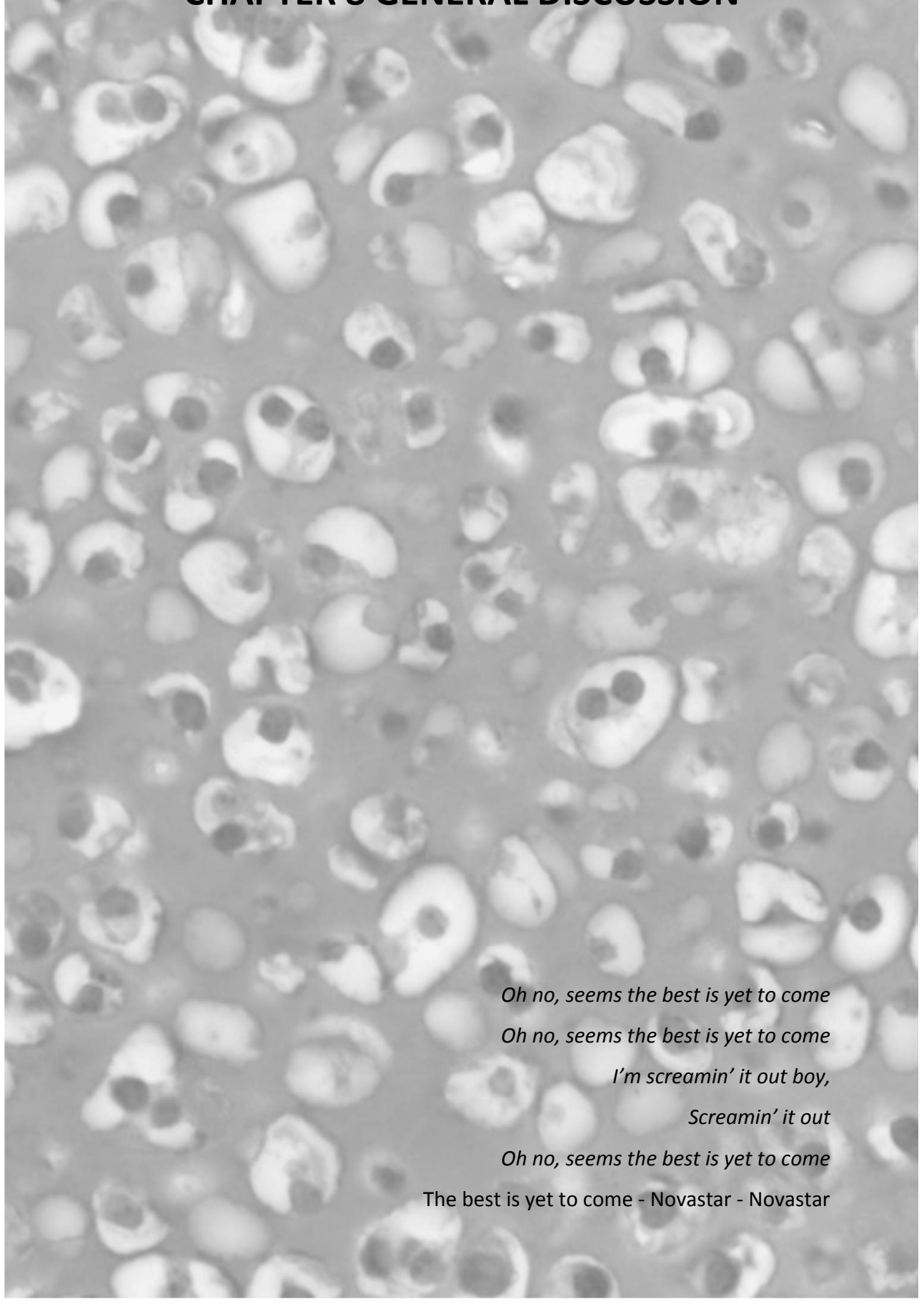




\section{GENERAL DISCUSSION}

\section{Future challenges for cartilage tissue engineering using embryonic stem cells}

Tissue engineers are facing common challenges, regardless of the tissue they are trying to repair. First, the cell type, the scaffold material and the culture and differentiation protocol are to be chosen. Upon further investigation, cell-scaffold interactions, such as cell attachment and distribution, survival of cells on or in the scaffolds, and possible instructive characteristics of the scaffold material will have to be fine-tuned. Finally, once the cells have formed the desired tissue, the stability of the tissue in vivo and the integration with the surrounding tissue are essential for successful clinical application.

Mouse embryonic stem cells (ESCs) were isolated in 1981 [10,11], followed by human ESCs in 1998 [12]. The first study describing directed chondrogenic differentiation of mouse ESCs was published in 2000 [87]. At the start of this thesis in 2003, chondrogenic differentiation of human ESCs and the potential of ESCs for cartilage tissue engineering had not been investigated. In this thesis, we have described that cartilage was formed by both mouse and human ESCs, but from our experiments we could conclude that the current protocols have to be improved before ESCs can be successfully and safely applied in cartilage tissue engineering. In this chapter, we discuss some of the challenges that are more or less specific for the application of ESCs in cartilage tissue engineering.

\section{Proliferation of ESCs}

Cartilage defects can be up to $12 \mathrm{~cm}^{2}$ large and 0.5-7 mm thick [232]. Tissue engineering approaches to repair cartilage defects generally require large amounts of chondrogenic cells: an average of 1 million cells $/ \mathrm{cm}^{2}$ for autologous chondrocyte transplantation [67], and a cell density ranging from roughly 5-50 million cells $/ \mathrm{cm}^{3}$ for scaffold-based strategies. The limited proliferative capacity and associated loss in differentiation potential of human chondrocytes and human mesenchymal stem cells (MSCs) might hinder their application in cartilage tissue engineering. ESCs on the other hand can proliferate indefinitely, but we have witnessed differences in the efficiency to obtain clinically relevant amount of ESCs. 
Proliferation of mouse ESC line iB10 proved to be straight forward, and the formation of EBs was very efficient. We were able to culture billions of EB cells with little effort in 1 week, which is sufficient to create a tissue-engineered cartilage implant. In contrast, experiments with human ESCS were seriously hampered by the low number of cells we were able to generate, in spite of the countless hours spent on cell culturing. In addition, we often encountered spontaneous differentiation of human ESCs, which sometimes resulted in barely enough undifferentiated colonies to maintain the culture. Also, the formation of human EBs is far less efficient than what we observed for mouse EBs. Starting with 20 dishes with 10-12 human ESC colonies each, we could only obtain a total of 2-4 million human EB cells. To put this into perspective, it took 2 entire days to upscale the human ESC culture from 20 to 60 to 120 dishes and another day of mechanical dissection to form human EBs. Subsequently, we obtained approximately 15 million cells to seed on scaffolds and some control samples for a small in vivo experiment. Obtaining clinically relevant amounts, e.g. 50 million human EB cells, using the current proliferation protocol will be very labor intensive and timeconsuming, and may even be near impossible.

In the past years, researchers have attempted to optimize the proliferation protocols on several points. Standard protocols for human ESC culture include growth on a feeder layer of mouse embryonic fibroblasts, and mechanical dissection of the colonies. Leukemia inhibitory factor, which could replace the feeder layer in mouse ESCS cultures, is insufficient to maintain the self-renewal potential of human ESCs. The mouse embryonic fibroblasts have been replaced by human feeders, which are more feasible for clinical applications, by ECM extracts such as Matrigel, or by conditioned medium [233]. Mouse ESCs can be routinely expanded using trypsin. Human ESC cultures have been enzymatically expanded, for example using trypsin or collagenase IV, albeit with concomitant genomic instability. As such, each improvement had its disadvantages, and most did not result in the desired time and cost efficient protocol that could be translated to large scale cultures. The increasing understanding of the basis of self-renewal of human ESCs might result in straightforward culture protocols.

\section{Differentiation of ESCs}

We observed that cartilage formation by mouse ESCs turned out to be very reproducible, whereas the chondrogenic differentiation of human ESCs was rather inefficient (Chapter 5) [102]. Nevertheless, differentiation was never homogeneous, and other tissue structures could be recognized in the samples. 


\section{The influence of EB formation}

Most ESC differentiation protocols start with the formation of EBs. In fact, EB formation was essential for cartilage formation in our experiments (Chapter 5). Mesenchymal condensation is one of the first steps in chondrogenesis. The aggregation of cells into EBs might mimic this step to some extent.

During EB formation, the ESCS form free-floating aggregates of randomly differentiating cells, expressing markers for all three germ layers within one EB [234]. The differentiation processes during this step are not well-characterized. Chondrogenic differentiation could only trigger a subset of the heterogeneous EB cell population to form cartilage, resulting in cartilage nodules, rather than homogeneous differentiation. One could hypothesize that the fate of an ESC to differentiate into a chondrocyte is determined during EB formation. The amount of cartilage nodules would then correlate with the amount of chondrogenic cells formed during EB formation. Clonal expansion of these cells or cell-cell interaction may have determined the size of the cartilage nodules. Factors can be added to the EB medium to reduce the randomness of differentiation during EB formation [146]. By either stimulating the differentiation towards mesodermal cells, or by reducing the differentiation towards ectoderm and endoderm, a higher percentage of prechondrogenic EBs might form, thereby improving the homogeneity of cartilage formation by ESCs.

Nevertheless, differentiation within an EB might be less random than expected, evidenced by full chondrogenic differentiation of a subset of intact EBs encapsulated in hydrogels [102]. If prechondrogenic EBs could be selected from the cultures, it would result in more homogeneous cartilage formation. Another interesting observation we made is that intact EBs survive better in hydrogels than single EB cells. In addition, cell-cell contact seemed beneficial for cartilage formation by mouse ESCs [102]. Further investigation is needed to elucidate whether the use of intact EBs rather than EB cells is beneficial for cartilage tissue engineering. Another advantage to use intact EBs would be the reduction of cells lost during the dissociation of human EBs into single cells.

\section{Chondrogenic medium}

In the developing embryo, many signals influence the cells during their differentiation towards chondrocytes. A combination of growth factors, cytokines, hormones, transcription factors, cell-cell and cell-matrix interactions defines cartilage formation.

Even though serum-free chondrogenic medium containing TGFß can be a good basis for cartilage formation by ESCs, it is not surprising that differentiation in medium with the same the composition throughout the entire differentiation from EB cell to chondrocyte does not result in homogeneous 
cartilage formation. Culturing in media with a specific cocktail of growth factors during each step might direct the pluripotent ESCs, via EB formation, into the mesodermal lineage, towards mesenchymal cells and ultimately chondrocytes. Another shortcoming of the current chondrogenic medium is that its components do not exclusively differentiate cells into the chondrogenic lineage. So far, no unique chondrogenic factor has been defined, but ESCs have been used as a model system to search for and identify these factors [78,84].

\section{Cell sorting}

More homogeneous cartilage formation can be achieved by cell sorting, using fluorescence- or magnetic-activated cell sorting (FACS or MACS). This can be either by selecting against cells that have not differentiated into the chondrogenic lineage, or by selecting for chondrogenic cells. The most efficient time for selection will have to be determined. Early sorting for a more general mesodermal marker [101] or mesenchymal marker [106] would result in a large fraction of cells, but due to their multipotency, they might still differentiate into other tissue types. On the other hand, sorting for a chondrocyte-specific marker will result in the most homogeneous chondrogenic cell population, but in this case the cells might have to be isolated from their cartilaginous matrix and the yield will be low. Even though an indefinite amount of ESCS will be available due to their ability to self-renew, millions of chondrogenic cells are required to engineer a cartilage construct. Therefore, a high yield is important for successful selection strategies in a clinical setting.

\section{Scaffolds}

We seeded ESCs onto polymeric scaffolds that have previously been studied by our lab for cartilage tissue engineering, and onto calcium phosphate scaffolds which have been used for bone tissue engineering. We investigated chondrogenic differentiation of ESCs in hydrogels that have been studied in cartilage research, such as agarose and alginate, and commercially available hydrogels such as Matrigel and Puramatrix. The project "An artificial extra cellular matrix for the regeneration of cartilage", which was the basis for the research described in this thesis, resulted in a newly developed dextran-based hydrogel $[170,171]$. We analyzed its potential for cartilage tissue engineering using both bovine chondrocytes and mouse ESCs (Chapter 3). ESCs formed cartilage in or on all scaffold materials we have investigated so far.

\section{Hydrogels for cartilage tissue engineering}

When designing a hydrogel for cartilage tissue engineering, some basic requirements have to be met. Obviously, cells should survive in the hydrogel. Therefore, gelation in the presence of radicals, 
high temperatures or photopolymerization under extreme wavelengths should be avoided to ensure cell survival during encapsulation. The hydrogel should be biocompatible and permeable to allow nutrient diffusion and removal of waste products. Upon formation of cartilage tissue, the hydrogel should degrade into non-toxic components which can be removed by the body.

When engineering a cartilage construct, it is desirable that the hydrogel can be formed in any desired shape and size, to match the cartilage defect contours. However, some gelation conditions limit the size and shape of the hydrogel, for example alginate which forms beads when immersed in a $\mathrm{CaCl}_{2}$ solution. Hydrogels are generally too soft to have good load bearing properties. Therefore, we have encapsulated cells in the hydrogel and subsequently injected this mixture into a polymeric scaffold for support.

A constant quality of the hydrogels, without batch variation, is important for reproducible results. Sterilization of the hydrogel components should not affect their properties. Functionalized groups on the hydrogel components might react to high temperatures or radiation, and some gel solutions are too viscous to pass through a filter.

\section{Hydrogels for cartilage tissue engineering using ESCS}

Some characteristics of the hydrogel might have to be adjusted when ESCS are used to create a cartilage implant. We have experienced that ESCs are more sensitive than chondrocytes, as seen by the decreased viability of EB cells in the hydrogels [102]. Therefore, the gelation conditions might have to be milder to ensure ESC survival. Alternatively, intact EBs could be encapsulated with increased survival rates. We observed that EBs would settle at the bottom of the hydrogel much faster than single cells. To prevent this inhomogeneous distribution, the gelation time should not be too long. When undifferentiated EB cells or intact EBs are encapsulated in the hydrogel, in vitro preculture will be required to avoid teratoma formation in vivo. In addition, cartilage formation by ESCs is delayed when compared to chondrocytes. Consequently, the degradation time of the gels might have to be increased.

\section{Cell-scaffold interactions}

We observed differences in cell-scaffold interactions and distribution of the formed cartilage matrix based on the scaffold used. The influence of the scaffold material on cell seeding and differentiation requires further investigation. 


\section{Cell seeding and scaffold architecture}

The mechanical properties of the 3D fiber deposited (3DF) scaffolds can be varied by changing the fiber spacing, the layer thickness and the orientation between successive layers $[181,182,235,236]$. In this thesis, we investigated the effect of various designs on the seeding homogeneity and efficiency of ESCs on the scaffolds. It was observed that EB cells easily flowed through the fully interconnected pores of the 3DF scaffolds. To retain the cells in the scaffolds, we adjusted the angle of the successive layers, deposited fibers in a staggered orientation or reduced the fiber spacing. Intact EBs on the other hand, were too large to infiltrate the scaffold and fiber spacing and layer thickness were increased to allow distribution of the EBs in the scaffolds. Although we could design the 3DF scaffolds to meet our requirements, the seeding efficiency was more influenced by the medium, than by the design of our scaffolds.

\section{Cell seeding in serum-free chondrogenic medium}

The absence of serum proteins in chondrogenic medium drastically reduced cell attachment to our polymeric scaffolds. We tried to increase the initial seeding efficiency by preincubating the scaffolds with serum and by seeding the EB cells in medium containing serum. However, subsequent culture in chondrogenic medium resulted in cell detachment from the fibers during prolonged incubation. Unfortunately, the addition of serum to the chondrogenic medium inhibited the formation of cartilage by ESCs (Chapter 5 ).

We observed that the seeding efficiency was higher under static conditions [102], but dynamic seeding in a spinner flask or in a tube on a roller bank resulted in increased homogeneity. It stresses the importance to improve the seeding conditions to achieve better seeding efficiency and homogeneity.

\section{Cell-material interaction}

We have observed differences in cell survival, cartilage formation and GAG distribution, which seemed to depend on cell adhesive properties of the hydrogels [102]. Cell attachment in Matrigel did not only seem to increase the viability, but also the chondrogenic differentiation of ESCs. Similarly, cartilage matrix deposition by human ESC-derived MSC-like cells increased in hydrogels modified with arginine-glycine-apsarate (RGD) peptides [109]. In contrast, integrin-mediated attachment of chondrocytes results in dedifferentiation and reduced matrix deposition [2]. Characterization of ESC-material [237] and chondrocyte-material interactions can identify how hydrogels can be functionalized with adhesion peptides to enhance cartilage formation. 


\section{ESC-derived cartilage in vivo}

An important requirement for successful application of ESCs in cartilage tissue engineering is the stability of the implanted cartilage. The engineered cartilage should integrate with healthy cartilage surrounding the defect and reorganize into the right zonal distribution.

\section{Stability of implanted cartilage}

When we implanted cartilage formed by ESCs in Matrigel, the hydrogel degraded, and most of the cartilage could not be retrieved after explantation [102]. We do not know whether the cartilage degraded with the gel, or if the superficial cartilage layer was disrupted while inserting the constructs into the subcutaneous pockets during implantation. Instability of engineered cartilage was also observed when MSCs, differentiated into the chondrogenic lineage for 3 weeks, were implanted into immuno-deficient mice. The cartilage either degraded, or fibrous or fibrocartilagelike implants were retrieved $[210,238]$. It appeared that longer in vitro incubation resulted in fewer degraded cartilage samples after implantation [238]. Thus, the stability of ESC-derived cartilage in vivo might be improved by changing the in vitro differentiation protocol.

\section{Endochondral ossification}

An unexpected, yet interesting form of cartilage instability was observed when we implanted ESCderived cartilage formed on ceramic or polymeric scaffolds. Bone was formed on this cartilage template via the process of endochondral ossification [129]. In the developing embryo the cartilaginous skeleton is replaced by bone and cartilage in the epiphyseal plates will mature, become hypertrophic and is ultimately replaced by bone, allowing bone growth. Permanent hyaline cartilage is found on the articular joint surface.

We have not yet investigated what triggers ESC-derived chondrocytes to follow the path of endochondral bone formation. It has been reported that ESC-derived cardiomyocytes tissue displayed characteristics of embryonic rather than adult tissue [239]. This might implicate that ESCderived cartilage will behave as embryonic tissue, and its fate is thus to be replaced by bone.

Further characterization of the ESC-derived cartilage will establish whether embryonic or adult, such as articular or growth plate, cartilage markers are expressed.

\section{Inhibition of cartilage maturation}

If we would like to use ESCs for cartilage tissue engineering, we have to prevent cartilage maturation into hypertrophy in vitro and in vivo. We did not observe an increase in the expression of the gene encoding collagen type $\mathrm{X}$, a marker for hypertrophy, after 21 days of in vitro chondrogenic 
differentiation. However, in the in vitro samples we sometimes observed chondrocytes in large lacunae surrounded by a thin layer of cartilage ECM, a morphology observed in hypertrophic cartilage. It has been reported by other groups that mouse and human ESCs express hypertrophic markers and even some osteogenic markers in vitro $[88,101,155]$. Therefore, we would have to further investigate if the ESC-derived cartilage already displayed signs of hypertrophy before implantation.

If so, hypertrophy should be suppressed. Parathyroid hormone-related peptide (PTHrP) is known to sustain chondrocyte proliferation and delay chondrocyte maturation [240]. Addition of PTHrP to chondrogenic pellet cultures of MSCs resulted in reduced expression of the collagen type $\mathrm{X}$ gene, and reduced alkaline phosphatase activity. Hypertrophy could be suppressed and chondrogenesis was even stimulated in the presence of PTHrP [241,242]. The effect of PTHrP on ESC chondrogenesis cultures has not been investigated yet, but modification of the in vitro culture protocol might result in cartilage with a matrix containing collagen type II but not collagen type $\mathrm{X}$.

\section{The effect of the environment}

Besides the cells and the scaffold, the environment plays an important role in the stability of the implanted cartilage and hence successful cartilage tissue engineering.

In our studies, we implanted ESC-derived cartilage subcutaneously in immuno-deficient mice. This environment is not the same as a cartilage defect and cells are exposed to different biological and mechanical influences. A drawback of the current animal models, i.e. immuno-deficient mice and rats, is the small size of the cartilage defect that can be created. Implantation in these small defects remains limited to small constructs, such as pellets. As such, characteristics associated with larger constructs, such as nutrient limitation, cannot be investigated. The cartilage layer in mice and rat knees is very thin, which complicates the creation of a cartilage defect without disturbing the subchondral bone.

\section{Teratoma formation}

The propensity of undifferentiated ESCs to develop teratomas after injection into mouse organs is used to demonstrate the pluripotency of newly isolated ESCs. Even though teratomas are benign tumors, it is obvious that they are highly undesirable in a clinical setting. Upon differentiation, ESCS will loose their capacity to form teratomas. The incidence of teratoma formation appears to decrease with increasing in vitro differentiation times. Prolonged in vitro differentiation of human ESCs into the neuronal lineage was essential to prevent the formation of teratomas [243]. We observed more and larger teratomas after 7 days of preculture in vitro, when compared to 14 and 
21 days. However, after in vitro chondrogenic differentiation for 21 days, cartilage was formed by ESCs, but Oct4, a marker for undifferentiated cells, was still expressed by another fraction of the cells in the samples [244], and teratomas rapidly developed upon subcutaneous implantation into immuno-deficient mice [102].

Obviously, the number of implanted undifferentiated cells corresponds to the chance of teratoma formation. Studies describing the injection of undifferentiated ESCs into the joints of mice and rats have used samples of 10-15 $\mu \mathrm{l}$ with a cell density of 10 million cells/ml [162-164]. Even though we will implant differentiated cells, the high cell numbers required to create a cartilage implant will increase the chance of introducing remaining undifferentiated cells into the knee. Therefore, it will be essential to demonstrate that no undifferentiated cells are implanted into a patient. This can be either achieved by ensuring that all cells will differentiate, or by removing the undifferentiated cell before implantation.

Implantation in the tissue of interest does not prevent teratoma formation of undifferentiated ESCs, as observed when ESCs were injected into the heart [245,246] or liver [212]. Mouse ESC injected into a mouse joint formed teratomas and destroyed the joint, although the incidence was lower and teratomas were smaller than teratomas developing after subcutaneous injection [162]. Teratoma formation was not observed when mouse ESCs were implanted into an osteochondral defect in a rat joint [163]. However, when the joints were immobilized, large teratomas still developed in the defects [164]. It seems that a confined space such as the joint compared to a subcutaneous pocket or the immobilized joint versus the free motion joint, reduced the incidence of teratoma formation. When we implanted chondrogenically differentiated ESCs into a cranial defect, we still observed teratoma formation, sometimes leading to the death of the rats [247]. In conclusion, we cannot prevent teratoma formation by implanting the ESC-derived cartilage implant into a cartilage defect.

\section{ESCs: from the lab to the clinic?}

In the case that we are able to solve all the problems described above, can we transfer ESCs from the lab to the clinic?

\section{The law and embryonic stem cells}

Ever since the isolation of human ESCs in 1998 [12], there has been ethical debate on the use, creation and destruction of human embryos. In many countries, it is forbidden by law to use ESCs for research or to isolate new human ESC lines. In the Netherlands, the Embryo Law dating from 2002 does not allow the creation of embryos, but the isolation of human ESCs from leftover embryos for 
medical research is allowed. However, their medical application is not yet allowed. The government awaits new scientific insight to update this law.

The first FDA approval for a clinical study using human ESCs was given in January 2009. This is considered to be a milestone and researchers and companies are hoping this will lead to approval for more clinical studies. For the past 8 years, ESC research in the USA has been limited by presidential actions. In March 2009, the newly elected president Obama removed the limitations to fund and conduct human ESC research, which is expected to greatly boost ESC research in the USA.

\section{Patient-specific cells}

Because ESCS cannot be isolated from the patient, in contrast to bone marrow or adipose tissuederived stem cells, the problem of immuno-rejection should be solved. By creating a bank of ESCs to match the human leukocyte antigen (HLA) of ESC lines with the HLA of the patient's cells, and additional administration of immunosuppressants, the patient's body might accept the implanted ESC-derived cartilage construct.

An alternative may be the generation of induced pluripotent stem cells (iPS cells) from adult cells $[17,61,62,160,248]$. These iPS cells display many characteristics of ESCs, such as their self-renewal capacity and pluripotency, but unfortunately also their complicated culture protocols and capacity to form teratomas. Thus, these disadvantages of ESCs are currently not solved by using iPS cells to create a cartilage construct. However, advantages of iPS cells over ESCs include the ability to be generated from adult cells, thereby making it possible to create autologous pluripotent cells. In addition, the ethical controversy surrounding human ESCs does not apply to iPS cells. It is to be expected that future clinical applications of pluripotent stem cells will use iPS cells rather than ESCs.

\section{Conclusion}

A lot of research will be required before ESCs can be safely used to create a cartilage implant. Relatively few groups are investigating cartilage tissue engineering using ESCs. However, most of the challenges concerning the application of ESCs in a clinical setting are not cartilage-specific and as such, cartilage tissue engineers can benefit from other studies in which ESCs are investigated for their application in treatment of for example Alzheimer's disease or diabetes. Some of the challenges addressed in this chapter have not received ample attention, and some have been slightly ignored, such as the formation of teratomas after implantation of tissue-engineered samples. In addition, the translation of experimental data from mouse to human ESCs has not been as straightforward as expected, and the variation among various ESC lines will influence the reproducibility of studies. Up to this moment, the culture protocols are not suitable for large-scale 
culture. Nevertheless, in the past years, researchers have made a lot of progress, both on the characterization of ESCS and on cartilage formation. Our results on endochondral bone formation are promising for both developmental biologists and bone tissue engineers. As such, ESCs have proven to be a valuable model system, and the potential for application in skeletal tissue engineering has been established. 


\section{SUMMARY}

Tissue engineering aims at repairing or replacing damaged or diseased tissue. Cells, scaffolds, or both form the basis of this approach. Articular cartilage has a limited capacity for self-repair after injury. Tissue engineered cartilage might be used to repair cartilage defects. Chondrocytes can be isolated from articular cartilage, harvested from a minor load-bearing region of the knee, and expanded in vitro. The availability of chondrocytes is limited, and chondrocytes dedifferentiate during expansion. Furthermore, osteoarthritic chondrocytes have a disturbed metabolism. As such, chondrocytes might not be the ideal cell source to repair the defect.

Therefore, researchers investigated if stem cells can be used for cartilage tissue engineering. Stem cells can self-renew and differentiate into various cell types. The isolation and characteristics of embryonic and adult stem cells are described in Chapter 1 . In this thesis, we investigated the potential of embryonic stem cells (ESCs) for cartilage tissue engineering. After differentiation of mouse and human ESCs into the chondrogenic and osteogenic lineage had been established, tissue engineers focused on the formation of cartilage and bone by ESCs in combination with scaffolds (reviewed in Chapter 2).

Various scaffold materials have been investigated for cartilage tissue engineering, such as polymeric scaffolds and hydrogels. The high water content of hydrogels allows the encapsulation of cells and biological compounds. We investigated whether chondrocytes would form cartilage in a newly developed dextran-based hydrogel. These hydrogels formed in situ via a Michael type addition reaction by mixing a thiol-functionalized dextran (Dex-SH) and a tetra-acrylated star poly(ethyleneglycol) (PEG-4-Acr). The degradation time of the Dex-PEG hydrogel could be adjusted by changing the amounts of crosslinks. The chondrocytes survived gelation and formed cartilage extracellular matrix (Chapter 3). We also encapsulated aggregates of ESCs, so called embryoid bodies, in the DexPEG hydrogel, and cartilaginous tissue was formed.

We established some critical steps when using ESCs for cartilage tissue engineering (Chapter 4). 3D fiber deposited polymeric scaffolds have fully interconnected pores and can be designed to match the mechanical properties of cartilage. However, the seeding efficiency on the polymeric scaffold material (300PEOT55PBT45) used in our studies was low. ESCs attached poorly to the scaffold fibers due to the lack of serum proteins in the chondrogenic medium. The seeding efficiency and homogeneity could be increased by encapsulating cells in a hydrogel, such as agarose or Matrigel, 
and injecting this mixture into the polymeric scaffold. The viability of single ESCs in a hydrogel was unsatisfactory, but intact EBs could be incubated for prolonged times. Mouse EB cells and EBs formed cartilage in pellets, on polymeric scaffolds and in hydrogels, when cultured in vitro in serumfree chondrogenic medium with transforming growth factor-beta (TGFß). Cartilage was formed in nodules and other cell types were also observed. We attempted to improve the homogeneity of cartilage formation, and we examined the necessity of the components in the chondrogenic medium (Chapter 5). Whereas one mouse ESC line showed efficient and reproducible cartilage formation, other mouse ESC lines only formed small amounts of cartilage. We transferred the differentiation protocol from mouse ESCs to human ESCs, and observed that some cartilaginous tissue was formed, but with very low efficiency (Chapter 5).

When we implanted cartilage formed by mouse ESCs in hydrogels in polymeric scaffolds subcutaneously into the back of immuno-deficient mouse, we could retrieve some cartilage (Chapter 4). When we implanted cartilage formed by mouse ESCs on ceramic or polymeric scaffolds, we observed that the cartilage matured, became hypertrophic, calcified and was ultimately replaced by bone tissue in the course of 21 days (Chapter 6). Hence, the cartilage was used as a template for endochondral bone formation. This is the first report of in vivo bone formation using ESCs, both ectopically and orthotopically in a cranial defect, under controlled, reproducible conditions. Bone tissue engineering by directly differentiating ESCs into osteoblasts was unsuccessful so far. A future perspective of how endochondral bone tissue formation might be translated into a clinical product is described in Chapter 7.

Although we were able to take the first steps towards an ESC-derived cartilage implant, many challenges remain, especially for human ESCs. The proliferation and differentiation protocols have to be improved on various aspects. Cartilage formation has to be more homogeneous, and no remaining undifferentiated ESCs can be implanted, because this will result in the formation of benign tumors (teratomas). More in vivo studies are necessary to demonstrate the stability and safety of the implanted cartilage. Scaffold materials should be optimized, and most likely functionalized to enhance cartilage formation by ESCs. These challenges are discussed in Chapter 8. 


\section{SAMENVATTING}

Weefselkweek (tissue engineering) heeft als doel beschadigd of ziek weefsel te herstellen of vervangen. Cellen, dragermaterialen (scaffolds), of beide, vormen de basis van deze aanpak. Beschadigd kraakbeen kan zichzelf niet of nauwelijks herstellen. Gekweekt kraakbeen zou gebruikt kunnen worden om het kraakbeendefect te herstellen. Kraakbeencellen (chondrocyten) worden geïsoleerd uit kraakbeenweefsel dat geoogst wordt uit een gedeelte van de knie dat minder belast wordt, en vermeerderd in een kweekfles (in vitro). Er kan slechts weinig kraakbeenweefsel geoogst worden, en na de vermeerdering is het moeilijk de kraakbeencellen aan te zetten tot de vorming van kraakbeeen. Bovendien hebben kraakbeencellen van een osteoartritis patiënt een verstoorde stofwisseling. Kraakbeencellen zijn daardoor misschien niet de beste kandidaat om het kraakbeendefect te herstellen.

Wetenschappers hebben onderzocht of stamcellen toegepast kunnen worden in kraakbeenweefselkweek. Stamcellen kunnen zichzelf vernieuwen, en uitgroeien tot verschillende celtypes (differentiatie). De isolatie en karakterisering van embryonale en volwassen stamcellen is beschreven in Hoofdstuk 1. In dit proefschrift hebben we onderzocht of het mogelijk is embryonale stamcellen (ESCS) te gebruiken voor kraakbeenweefselkweek. Nadat was vastgesteld dat ESCS konden uitgroeien tot kraakbeen- en botcellen, richtten weefselkwekers hun aandacht op het vormen van kraakbeen- en botweefsel in combinatie met dragermaterialen (Literatuuroverzicht in Hoofdstuk 2).

Diverse dragermaterialen, zoals polymeren en hydrogelen, zijn onderzocht om er kraakbeenweefsel op te kweken. Omdat hydrogelen veel water bevatten, kunnen cellen en biologische actieve stoffen erin worden gemengd. In Hoofdstuk 3 hebben we onderzocht of kraakbeencellen in een nieuw ontwikkelde hydrogel op basis van dextraan kraakbeenweefsel vormden. Deze hydrogelen vormden onder fysiologische omstandigheden via een Michael type additie reactie tussen dextraan met een thiolgroep (Dex-SH) en poly(ethyleen glycol) tetra-acrylaat (PEG-4-Acr). De afbraaksnelheid van deze Dex-PEG hydrogel kon worden aangepast door het aantal verbindingen tussen dextraan en PEG te veranderen. De kraakbeencellen overleefden de vorming van de hydrogel, en vormden kraakbeen. We mengden ook celklompjes van ESCs, zogenaamde embryoid bodies (EBs), in de Dex-PEG gel, en kraakbenig weefsel werd gevormd. 
We hebben een aantal kritische stappen vastgesteld wanneer ESCs gebruikt worden voor kraakbeenweefselkweek (Hoofdstuk 4). 3D geprinte dragermaterialen kunnen zo ontworpen worden dat ze de mechanische eigenschappen van kraakbeen evenaren. Echter, de zaai-efficiëntie op het polymeer materiaal (300PEOT55PBT45) dat we in onze studies hebben gebruikt was laag. ESCS hechten zich slecht aan de polymeer fibers, door het gebrek aan serumeiwitten in het kraakbeenkweekmedium. De zaai-efficiëntie en zaaihomogeniteit konden verhoogd worden door cellen in een hydrogel, zoals agarose of Matrigel, te mixen en dit mengsel in het polymeer dragermateriaal te injecteren. De overleving van ESCs in de hydrogelen was onvoldoende, maar de celklompjes (EBS) konden wel langer gekweekt worden.

Muizen ESCs, zowel cellen als celklompjes, vormden kraakbeen in vitro in celpellets, op polymeer dragermaterialen en in hydrogelen wanneer ze gekweekt werden in kraakbeen differentiatiemedium zonder serum met de groeifactor TGFß. Kraakbeen werd gevormd in klontjes en daarnaast zagen we ook andere cel- en weefseltypes. We probeerden de homogeniteit van kraakbeenvorming te verbeteren, en we onderzochten welke componenten in het kraakbeen differentiatie-medium essentieel zijn (Hoofdstuk 5). Terwijl 1 van de muizen ESC lijnen efficiënt en reproduceerbaar kraakbeen vormde, vormden andere muizenlijnen slechts kleine hoeveelheden kraakbeen. Als we menselijke ESCs kweekten in kraakbeen differentiatie-medium zagen we dat er een beetje kraakbenig weefsel werd gevormd, maar met zeer lage efficiëntie (Hoofdstuk 5).

Wanneer we kraakbeen gevormd door muizen ESCs in hydrogelen in polymeer dragermaterialen onder de huid implanteerden in de rug van immuundeficiënte muizen (in vivo), konden we een beetje kraakbeen terugvinden (Hoofdstuk 4). Wanneer we kraakbeen gevormd door muizen ESCs op polymeer of keramische dragermaterialen implanteerden, zagen we dat het kraakbeen in de loop van 21 dagen volgroeide, hypertroof werd, verkalkte en uiteindelijk werd vervangen door botweefsel (Hoofdstuk 6). Het kraakbeen werd dus gebruikt als mal voor endochondrale botvorming. Dit is de eerste studie waarin in vivo bot werd gevormd met ESCs, zowel onderhuids als in een botdefect, onder gecontroleerde, reproduceerbare omstandigheden. Botvorming door ESC direct in botvormende cellen te differentiëren was tot nu toe niet succesvol. Een toekomstblik hoe endochondrale botvorming vertaald kan worden naar een klinisch product is beschreven in Hoofdstuk 7.

Ook al hebben we de eerste stappen kunnen zetten om te komen tot de vorming van een kraakbeenimplantaat met behulp van ESCs, blijven er nog veel uitdagingen over, zeker voor menselijke ESCs. De groei- en differentiatieprotocollen moeten op diverse aspecten verbeterd 
worden. Kraakbeenvorming dient homogener te zijn, en ongedifferentieerde cellen mogen niet geïmplanteerd worden, omdat dit resulteert in de vorming van goedaardige tumoren (teratomas). Meer in vivo studies zijn nodig om de stabiliteit en veiligheid van het geïmplanteerde kraakbeen aan te tonen. Dragermaterialen moeten worden geoptimaliseerd, en hoogstwaarschijnlijk dienen er extra eigenschappen aan toegevoegd te worden, om kraakbeenvorming te verbeteren. Deze uitdagingen zijn beschreven in Hoofdstuk 8. 


\section{REFERENCES}

[1] Morrison, SJ and Kimble, J. Asymmetric and symmetric stem-cell divisions in development and cancer. Nature 2006;441:1068-74

[2] von der Mark, K, Gauss, V, von der Mark, H and Muller, P. Relationship between cell shape and type of collagen synthesised as chondrocytes lose their cartilage phenotype in culture. Nature 1977;267:531-2

[3] Slack, JM and Tosh, D. Transdifferentiation and metaplasia--switching cell types. Curr Opin Genet Dev 2001;11:581-6

[4] Holden, C and Vogel, G. Stem cells. Plasticity: time for a reappraisal? Science 2002;296:2126-9

[5] Wagers, AJ and Weissman, IL. Plasticity of adult stem cells. Cell 2004;116:639-48

[6] Terada, N, Hamazaki, T, Oka, M, Hoki, M, Mastalerz, DM, Nakano, Y, Meyer, EM, Morel, L, Petersen, BE and Scott, EW. Bone marrow cells adopt the phenotype of other cells by spontaneous cell fusion. Nature 2002;416:542-5

[7] Ying, QL, Nichols, J, Evans, EP and Smith, AG. Changing potency by spontaneous fusion. Nature 2002;416:545-8

[8] Jackson, KA, Snyder, DS and Goodell, MA. Skeletal muscle fiber-specific green autofluorescence: potential for stem cell engraftment artifacts. Stem Cells 2004;22:180-7

[9] Adcock, IM, Ford, P, Barnes, PJ and Ito, K. Epigenetics and airways disease. Respir Res 2006;7:21

[10] Evans, MJ and Kaufman, MH. Establishment in culture of pluripotential cells from mouse embryos. Nature 1981;292:154-6

[11] Martin, GR. Isolation of a pluripotent cell line from early mouse embryos cultured in medium conditioned by teratocarcinoma stem cells. Proc Natl Acad Sci U S A 1981;78:7634-8

[12] Thomson, JA, Itskovitz-Eldor, J, Shapiro, SS, Waknitz, MA, Swiergiel, JJ, Marshall, VS and Jones, JM. Embryonic stem cell lines derived from human blastocysts. Science 1998;282:1145-7

[13] Williams, RL, Hilton, DJ, Pease, S, Willson, TA, Stewart, CL, Gearing, DP, Wagner, EF, Metcalf, D, Nicola, NA and Gough, NM. Myeloid leukaemia inhibitory factor maintains the developmental potential of embryonic stem cells. Nature 1988;336:684-7

[14] Odorico, JS, Kaufman, DS and Thomson, JA. Multilineage differentiation from human embryonic stem cell lines. Stem Cells 2001;19:193-204

[15] Hoffman, LM and Carpenter, MK. Human embryonic stem cell stability. Stem Cell Rev 2005;1:139-44

[16] Reubinoff, BE, Pera, MF, Fong, CY, Trounson, A and Bongso, A. Embryonic stem cell lines from human blastocysts: somatic differentiation in vitro. Nat Biotechnol 2000;18:399-404

[17] Maherali, N, Sridharan, r., Xie, W., Utikal, J., Eminli, S., Arnold, K., Stadtfeld, M., Yachechko, R., Tchieu, J., Jaenisch, R., Plath, K., Hochedlinger, K. Directly Reprogrammed Fibroblasts Show Global Epigenetic Remodeling and Widespread Tissue Contribution. Cell Stem Cell 2007;1:55-70

[18] Ying, QL, Nichols, J, Chambers, I and Smith, A. BMP induction of Id proteins suppresses differentiation and sustains embryonic stem cell self-renewal in collaboration with STAT3. Cell 2003;115:281-92

[19] Chambers, I, Colby, D, Robertson, M, Nichols, J, Lee, S, Tweedie, S and Smith, A. Functional expression cloning of Nanog, a pluripotency sustaining factor in embryonic stem cells. Cell 2003;113:643-55

[20] Mitsui, K, Tokuzawa, Y, Itoh, H, Segawa, K, Murakami, M, Takahashi, K, Maruyama, M, Maeda, M and Yamanaka, S. The homeoprotein Nanog is required for maintenance of pluripotency in mouse epiblast and ES cells. Cell 2003;113:631-42

[21] Pan, G and Thomson, JA. Nanog and transcriptional networks in embryonic stem cell pluripotency. Cell Res 2007;17:42-9

[22] Daheron, L, Opitz, SL, Zaehres, H, Lensch, WM, Andrews, PW, Itskovitz-Eldor, J and Daley, GQ. LIF/STAT3 signaling fails to maintain self-renewal of human embryonic stem cells. Stem Cells 2004;22:770-8

[23] Amit, M, Shariki, C, Margulets, V and Itskovitz-Eldor, J. Feeder layer- and serum-free culture of human embryonic stem cells. Biol Reprod 2004;70:837-45

[24] Carpenter, MK, Inokuma, MS, Denham, J, Mujtaba, T, Chiu, CP and Rao, MS. Enrichment of neurons and neural precursors from human embryonic stem cells. Exp Neurol 2001;172:383-97

[25] Zhang, SC, Wernig, M, Duncan, ID, Brustle, O and Thomson, JA. In vitro differentiation of transplantable neural precursors from human embryonic stem cells. Nat Biotechnol 2001;19:1129-33

[26] Reubinoff, BE, Itsykson, P, Turetsky, T, Pera, MF, Reinhartz, E, Itzik, A and Ben-Hur, T. Neural progenitors from human embryonic stem cells. Nat Biotechnol 2001;19:1134-40

[27] Mummery, C, Ward-van Oostwaard, D, Doevendans, P, Spijker, R, van den Brink, S, Hassink, R, van der Heyden, M, Opthof, T, Pera, M, de la Riviere, AB, Passier, R and Tertoolen, L. Differentiation of human 
embryonic stem cells to cardiomyocytes: role of coculture with visceral endoderm-like cells. Circulation 2003;107:2733-40

[28] He, JQ, Ma, Y, Lee, Y, Thomson, JA and Kamp, TJ. Human embryonic stem cells develop into multiple types of cardiac myocytes: action potential characterization. Circ Res 2003;93:32-9

[29] Rambhatla, L, Chiu, CP, Kundu, P, Peng, Y and Carpenter, MK. Generation of hepatocyte-like cells from human embryonic stem cells. Cell Transplant 2003;12:1-11

[30] Lavon, N, Yanuka, O and Benvenisty, N. Differentiation and isolation of hepatic-like cells from human embryonic stem cells. Differentiation 2004;72:230-8

[31] Assady, S, Maor, G, Amit, M, Itskovitz-Eldor, J, Skorecki, KL and Tzukerman, M. Insulin production by human embryonic stem cells. Diabetes 2001;50:1691-7

[32] Levenberg, S, Golub, JS, Amit, M, Itskovitz-Eldor, J and Langer, R. Endothelial cells derived from human embryonic stem cells. Proc Natl Acad Sci U S A 2002;99:4391-6

[33] Kaufman, DS, Hanson, ET, Lewis, RL, Auerbach, R and Thomson, JA. Hematopoietic colony-forming cells derived from human embryonic stem cells. Proc Natl Acad Sci U S A 2001;98:10716-21

[34] Chadwick, K, Wang, L, Li, L, Menendez, P, Murdoch, B, Rouleau, A and Bhatia, M. Cytokines and BMP-4 promote hematopoietic differentiation of human embryonic stem cells. Blood 2003;102:906-15

[35] Vats, A, Bielby, RC, Tolley, N, Dickinson, SC, Boccaccini, AR, Hollander, AP, Bishop, AE and Polak, JM. Chondrogenic differentiation of human embryonic stem cells: the effect of the micro-environment. Tissue Eng 2006;12:1687-97

[36] Levenberg, S, Huang, NF, Lavik, E, Rogers, AB, Itskovitz-Eldor, J and Langer, R. Differentiation of human embryonic stem cells on three-dimensional polymer scaffolds. Proc Natl Acad Sci U S A 2003;100:12741-6

[37] Martin, MJ, Muotri, A, Gage, F and Varki, A. Human embryonic stem cells express an immunogenic nonhuman sialic acid. Nat Med 2005;11:228-32

[38] Thomson, JA, Kalishman, J, Golos, TG, Durning, M, Harris, CP, Becker, RA and Hearn, JP. Isolation of a primate embryonic stem cell line. Proc Natl Acad Sci U S A 1995;92:7844-8

[39] Suemori, H, Tada, T, Torii, R, Hosoi, Y, Kobayashi, K, Imahie, H, Kondo, Y, Iritani, A and Nakatsuji, N. Establishment of embryonic stem cell lines from cynomolgus monkey blastocysts produced by IVF or ICSI. Dev Dyn 2001;222:273-9

[40] Thomson, JA, Kalishman, J, Golos, TG, Durning, M, Harris, CP and Hearn, JP. Pluripotent cell lines derived from common marmoset (Callithrix jacchus) blastocysts. Biol Reprod 1996;55:254-9

[41] Till, JE and Mc, CE. A direct measurement of the radiation sensitivity of normal mouse bone marrow cells. Radiat Res 1961;14:213-22

[42] Becker, AJ, Mc, CE and Till, JE. Cytological demonstration of the clonal nature of spleen colonies derived from transplanted mouse marrow cells. Nature 1963;197:452-4

[43] Friedenstein, AJ, Chailakhjan, RK and Lalykina, KS. The development of fibroblast colonies in monolayer cultures of guinea-pig bone marrow and spleen cells. Cell Tissue Kinet 1970;3:393-403

[44] Rando, TA. Stem cells, ageing and the quest for immortality. Nature 2006;441:1080-6

[45] Haynesworth, SE, Goshima, J, Goldberg, VM and Caplan, Al. Characterization of cells with osteogenic potential from human marrow. Bone 1992;13:81-8

[46] de Bruijn, JD, van den Brink, I, Mendes, S, Dekker, R, Bovell, YP and van Blitterswijk, CA. Bone induction by implants coated with cultured osteogenic bone marrow cells. Adv Dent Res 1999;13:74-81

[47] Dezawa, M, Ishikawa, H, Itokazu, Y, Yoshihara, T, Hoshino, M, Takeda, S, Ide, C and Nabeshima, Y. Bone marrow stromal cells generate muscle cells and repair muscle degeneration. Science 2005;309:314-7

[48] Park, IK, Qian, D, Kiel, M, Becker, MW, Pihalja, M, Weissman, IL, Morrison, SJ and Clarke, MF. Bmi-1 is required for maintenance of adult self-renewing haematopoietic stem cells. Nature 2003;423:302-5

[49] Molofsky, AV, Pardal, R and Morrison, SJ. Diverse mechanisms regulate stem cell self-renewal. Curr Opin Cell Biol 2004;16:700-7

[50] Schofield, R. The relationship between the spleen colony-forming cell and the haemopoietic stem cell. Blood Cells 1978;4:7-25

[51] Ohlstein, B, Kai, T, Decotto, E and Spradling, A. The stem cell niche: theme and variations. Curr Opin Cell Biol 2004;16:693-9

[52] Harley, CB, Futcher, $\mathrm{AB}$ and Greider, CW. Telomeres shorten during ageing of human fibroblasts. Nature 1990;345:458-60

[53] Hayflick, L. The Limited in Vitro Lifetime of Human Diploid Cell Strains. Exp Cell Res 1965;37:614-36

[54] Greider, CW and Blackburn, EH. Identification of a specific telomere terminal transferase activity in Tetrahymena extracts. Cell 1985;43:405-13 
[55] Simonsen, JL, Rosada, C, Serakinci, N, Justesen, J, Stenderup, K, Rattan, SI, Jensen, TG and Kassem, M. Telomerase expression extends the proliferative life-span and maintains the osteogenic potential of human bone marrow stromal cells. Nat Biotechnol 2002;20:592-6

[56] Pittenger, MF, Mackay, AM, Beck, SC, Jaiswal, RK, Douglas, R, Mosca, JD, Moorman, MA, Simonetti, DW, Craig, S and Marshak, DR. Multilineage potential of adult human mesenchymal stem cells. Science 1999;284:143-7

[57] Wilmut, I, Schnieke, AE, McWhir, J, Kind, AJ and Campbell, KH. Viable offspring derived from fetal and adult mammalian cells. Nature 1997;385:810-3

[58] Cowan, CA, Atienza, J, Melton, DA and Eggan, K. Nuclear reprogramming of somatic cells after fusion with human embryonic stem cells. Science 2005;309:1369-73

[59] Tada, M, Takahama, Y, Abe, K, Nakatsuji, N and Tada, T. Nuclear reprogramming of somatic cells by in vitro hybridization with ES cells. Curr Biol 2001;11:1553-8

[60] Takahashi, K and Yamanaka, S. Induction of pluripotent stem cells from mouse embryonic and adult fibroblast cultures by defined factors. Cell 2006;126:663-76

[61] Wernig, M, Meissner, A, Foreman, R, Brambrink, T, Ku, M, Hochedlinger, K, Bernstein, BE and Jaenisch, $\mathrm{R}$. In vitro reprogramming of fibroblasts into a pluripotent ES-cell-like state. Nature 2007;

[62] Okita, K, Ichisaka, T and Yamanaka, S. Generation of germline-competent induced pluripotent stem cells. Nature 2007;

[63] Adewumi, O, Aflatoonian, B, Ahrlund-Richter, L, Amit, M, Andrews, PW, Beighton, G, Bello, PA, Benvenisty, N, Berry, LS, Bevan, S, Blum, B, Brooking, J, Chen, KG, Choo, AB, Churchill, GA, Corbel, M, Damjanov, I, Draper, JS, Dvorak, P, Emanuelsson, K, Fleck, RA, Ford, A, Gertow, K, Gertsenstein, $M$, Gokhale, PJ, Hamilton, RS, Hampl, A, Healy, LE, Hovatta, O, Hyllner, J, Imreh, MP, Itskovitz-Eldor, J, Jackson, J, Johnson, JL, Jones, M, Kee, K, King, BL, Knowles, BB, Lako, M, Lebrin, F, Mallon, BS, Manning, D, Mayshar, Y, McKay, RD, Michalska, AE, Mikkola, M, Mileikovsky, M, Minger, SL, Moore, HD, Mummery, CL, Nagy, A, Nakatsuji, N, O'Brien C, M, Oh, SK, Olsson, C, Otonkoski, T, Park, KY, Passier, R, Patel, H, Patel, M, Pedersen, R, Pera, MF, Piekarczyk, MS, Pera, RA, Reubinoff, BE, Robins, AJ, Rossant, J, Rugg-Gunn, P, Schulz, TC, Semb, H, Sherrer, ES, Siemen, H, Stacey, GN, Stojkovic, M, Suemori, H, Szatkiewicz, J, Turetsky, T, Tuuri, T, van den Brink, S, Vintersten, K, Vuoristo, S, Ward, D, Weaver, TA, Young, LA and Zhang, W. Characterization of human embryonic stem cell lines by the International Stem Cell Initiative. Nat Biotechnol 2007;25:803-16

[64] Brittberg, M, Lindahl, A, Nilsson, A, Ohlsson, C, Isaksson, O and Peterson, L. Treatment of deep cartilage defects in the knee with autologous chondrocyte transplantation. N Engl J Med 1994;331:889-95

[65] Brittberg, M, Tallheden, T, Sjogren-Jansson, B, Lindahl, A and Peterson, L. Autologous chondrocytes used for articular cartilage repair: an update. Clin Orthop Relat Res 2001;\$337-48

[66] Peterson, L, Brittberg, M, Kiviranta, I, Akerlund, EL and Lindahl, A. Autologous chondrocyte transplantation. Biomechanics and long-term durability. Am J Sports Med 2002;30:2-12

[67] Tallheden, T, Bengtsson, C, Brantsing, C, Sjogren-Jansson, E, Carlsson, L, Peterson, L, Brittberg, M and Lindahl, A. Proliferation and differentiation potential of chondrocytes from osteoarthritic patients. Arthritis Res Ther 2005; 7:R560-8

[68] Zuk, PA, Zhu, M, Ashjian, P, De Ugarte, DA, Huang, Jl, Mizuno, H, Alfonso, ZC, Fraser, JK, Benhaim, P and Hedrick, MH. Human adipose tissue is a source of multipotent stem cells. Mol Biol Cell 2002;13:4279-95

[69] Campagnoli, C, Roberts, IA, Kumar, S, Bennett, PR, Bellantuono, I and Fisk, NM. Identification of mesenchymal stem/progenitor cells in human first-trimester fetal blood, liver, and bone marrow. Blood 2001;98:2396-402

[70] Zvaifler, NJ, Marinova-Mutafchieva, L, Adams, G, Edwards, CJ, Moss, J, Burger, JA and Maini, RN. Mesenchymal precursor cells in the blood of normal individuals. Arthritis Res 2000;2:477-88

[71] Romanov, YA, Svintsitskaya, VA and Smirnov, VN. Searching for alternative sources of postnatal human mesenchymal stem cells: candidate MSC-like cells from umbilical cord. Stem Cells 2003;21:105-10

[72] Fukuchi, Y, Nakajima, H, Sugiyama, D, Hirose, I, Kitamura, T and Tsuji, K. Human placenta-derived cells have mesenchymal stem/progenitor cell potential. Stem Cells 2004;22:649-58

[73] Kunisaki, SM, Jennings, RW and Fauza, DO. Fetal cartilage engineering from amniotic mesenchymal progenitor cells. Stem Cells Dev 2006;15:245-53

[74] De Coppi, P, Bartsch, G, Jr., Siddiqui, MM, Xu, T, Santos, CC, Perin, L, Mostoslavsky, G, Serre, AC, Snyder, EY, Yoo, JJ, Furth, ME, Soker, S and Atala, A. Isolation of amniotic stem cell lines with potential for therapy. Nat Biotechnol 2007; 25:100-6

[75] Kim, MS, Hwang, NS, Lee, J, Kim, TK, Leong, K, Shamblott, MJ, Gearhart, J and Elisseeff, J. Musculoskeletal differentiation of cells derived from human embryonic germ cells. Stem Cells 2005;23:113-23 
[76] Heng, BC, Cao, T and Lee, EH. Directing stem cell differentiation into the chondrogenic lineage in vitro. Stem Cells 2004;22:1152-67

[77] Heng, BC, Cao, T, Stanton, LW, Robson, P and Olsen, B. Strategies for directing the differentiation of stem cells into the osteogenic lineage in vitro. J Bone Miner Res 2004;19:1379-94

[78] Bi, W, Deng, JM, Zhang, Z, Behringer, RR and de Crombrugghe, B. Sox9 is required for cartilage formation. Nat Genet 1999;22:85-9

[79] Hargus, G, Kist, R, Kramer, J, Gerstel, D, Neitz, A, Scherer, G and Rohwedel, J. Loss of Sox9 function results in defective chondrocyte differentiation of mouse embryonic stem cells in vitro. Int J Dev Biol 2008;52:323-32

[80] Kim, JH, Do, HJ, Yang, HM, Oh, JH, Choi, SJ, Kim, DK, Cha, KY and Chung, HM. Overexpression of SOX9 in mouse embryonic stem cells directs the immediate chondrogenic commitment. Exp Mol Med 2005;37:261-8

[81] Tai, G, Polak, JM, Bishop, AE, Christodoulou, I and Buttery, LD. Differentiation of osteoblasts from murine embryonic stem cells by overexpression of the transcriptional factor osterix. Tissue Eng 2004;10:1456-66

[82] Tai, G, Christodoulou, I, Bishop, AE and Polak, JM. Use of green fluorescent fusion protein to track activation of the transcription factor osterix during early osteoblast differentiation. Biochem Biophys Res Commun 2005;333:1116-22

[83] Woei Ng, K, Speicher, T, Dombrowski, C, Helledie, T, Haupt, LM, Nurcombe, V and Cool, SM. Osteogenic differentiation of murine embryonic stem cells is mediated by fibroblast growth factor receptors. Stem Cells Dev 2007;16:305-18

[84] Ikeda, T, Kamekura, S, Mabuchi, A, Kou, I, Seki, S, Takato, T, Nakamura, K, Kawaguchi, H, Ikegawa, S and Chung, UI. The combination of SOX5, SOX6, and SOX9 (the SOX trio) provides signals sufficient for induction of permanent cartilage. Arthritis Rheum 2004;50:3561-73

[85] Ohba, S, Ikeda, T, Kugimiya, F, Yano, F, Lichtler, AC, Nakamura, K, Takato, T, Kawaguchi, H and Chung, UI. Identification of a potent combination of osteogenic genes for bone regeneration using embryonic stem (ES) cell-based sensor. Faseb J 2007;21:1777-87

[86] Cserjesi, P, Brown, D, Ligon, KL, Lyons, GE, Copeland, NG, Gilbert, DJ, Jenkins, NA and Olson, EN. Scleraxis: a basic helix-loop-helix protein that prefigures skeletal formation during mouse embryogenesis. Development 1995;121:1099-110

[87] Kramer, J, Hegert, C, Guan, K, Wobus, AM, Muller, PK and Rohwedel, J. Embryonic stem cell-derived chondrogenic differentiation in vitro: activation by BMP-2 and BMP-4. Mech Dev 2000;92:193-205

[88] Kramer, J, Hegert, C and Rohwedel, J. In vitro differentiation of mouse ES cells: bone and cartilage. Methods Enzymol 2003;365:251-68

[89] Kramer, J, Klinger, M, Kruse, C, Faza, M, Hargus, G and Rohwedel, J. Ultrastructural analysis of mouse embryonic stem cell-derived chondrocytes. Anat Embryol (Berl) 2005;210:175-85

[90] Kramer, J, Hegert, C, Hargus, G and Rohwedel, J. Mouse ES cell lines show a variable degree of chondrogenic differentiation in vitro. Cell Biol Int 2005;29:139-46

[91] Sui, Y, Clarke, T and Khillan, JS. Limb bud progenitor cells induce differentiation of pluripotent embryonic stem cells into chondrogenic lineage. Differentiation 2003;71:578-85

[92] Hwang, NS, Varghese, S and Elisseeff, J. Derivation of chondrogenically-committed cells from human embryonic cells for cartilage tissue regeneration. PLoS ONE 2008;3:e2498

[93] Kawaguchi, J, Mee, PJ and Smith, AG. Osteogenic and chondrogenic differentiation of embryonic stem cells in response to specific growth factors. Bone 2005;36:758-69

[94] zur Nieden, NI, Kempka, G, Rancourt, DE and Ahr, HJ. Induction of chondro-, osteo- and adipogenesis in embryonic stem cells by bone morphogenetic protein-2: effect of cofactors on differentiating lineages. BMC Dev Biol 2005;5:1

[95] Zanetti, NC and Solursh, M. Induction of chondrogenesis in limb mesenchymal cultures by disruption of the actin cytoskeleton. J Cell Biol 1984;99:115-23

[96] Zhang, Z, Messana, J, Hwang, NS and Elisseeff, JH. Reorganization of actin filaments enhances chondrogenic differentiation of cells derived from murine embryonic stem cells. Biochem Biophys Res Commun 2006;348:421-7

[97] Johnstone, B, Hering, TM, Caplan, Al, Goldberg, VM and Yoo, JU. In vitro chondrogenesis of bone marrow-derived mesenchymal progenitor cells. Exp Cell Res 1998;238:265-72

[98] Tanaka, H, Murphy, CL, Murphy, C, Kimura, M, Kawai, S and Polak, JM. Chondrogenic differentiation of murine embryonic stem cells: effects of culture conditions and dexamethasone. J Cell Biochem 2004;93:454-62

[99] Mackay, AM, Beck, SC, Murphy, JM, Barry, FP, Chichester, CO and Pittenger, MF. Chondrogenic differentiation of cultured human mesenchymal stem cells from marrow. Tissue Eng 1998;4:415-28 
[100] Yoo, JU, Barthel, TS, Nishimura, K, Solchaga, L, Caplan, Al, Goldberg, VM and Johnstone, B. The chondrogenic potential of human bone-marrow-derived mesenchymal progenitor cells. J Bone Joint Surg Am 1998;80:1745-57

[101] Nakayama, N, Duryea, D, Manoukian, R, Chow, G and Han, CY. Macroscopic cartilage formation with embryonic stem-cell-derived mesodermal progenitor cells. J Cell Sci 2003;116:2015-28

[102] Jukes, JM, Moroni, L, van Blitterswijk, CA and de Boer, J. Critical Steps toward a tissue-engineered cartilage implant using embryonic stem cells. Tissue Eng Part A 2008;14:135-47

[103] Toh, WS, Yang, Z, Liu, H, Heng, BC, Lee, EH and Cao, T. Effects of culture conditions and bone morphogenetic protein 2 on extent of chondrogenesis from human embryonic stem cells. Stem Cells 2007;25:950-60

[104] Koay, EJ, Hoben, GM and Athanasiou, KA. Tissue engineering with chondrogenically differentiated human embryonic stem cells. Stem Cells 2007;25:2183-90

[105] Koay, EJ and Athanasiou, KA. Hypoxic chondrogenic differentiation of human embryonic stem cells enhances cartilage protein synthesis and biomechanical functionality. Osteoarthritis Cartilage 2008;

[106] Barberi, T, Willis, LM, Socci, ND and Studer, L. Derivation of multipotent mesenchymal precursors from human embryonic stem cells. PLoS Med 2005;2:e161

[107] Lee, G, Kim, H, Elkabetz, Y, Al Shamy, G, Panagiotakos, G, Barberi, T, Tabar, V and Studer, L. Isolation and directed differentiation of neural crest stem cells derived from human embryonic stem cells. Nat Biotechnol 2007;25:1468-75

[108] Olivier, EN, Rybicki, AC and Bouhassira, EE. Differentiation of human embryonic stem cells into bipotent mesenchymal stem cells. Stem Cells 2006;24:1914-22

[109] Hwang, NS, Varghese, S, Zhang, Z and Elisseeff, J. Chondrogenic differentiation of human embryonic stem cell-derived cells in arginine-glycine-aspartate-modified hydrogels. Tissue Eng 2006;12:2695-706

[110] Fecek, C, Yao, D, Kacorri, A, Vasquez, A, Iqbal, S, Sheikh, H, Svinarich, DM, Perez-Cruet, M and Chaudhry, GR. Chondrogenic Derivatives of Embryonic Stem Cells Seeded into 3D Polycaprolactone Scaffolds Generated Cartilage Tissue In Vivo. Tissue Eng Part A 2008;

[111] Hwang, NS, Kim, MS, Sampattavanich, S, Baek, JH, Zhang, Z and Elisseeff, J. The Effects of Three Dimensional Culture and Growth Factors on the Chondrogenic Differentiation of Murine Embryonic Stem Cells. Stem Cells 2005;

[112] Hwang, NS, Varghese, S, Theprungsirikul, P, Canver, A and Elisseeff, J. Enhanced chondrogenic differentiation of murine embryonic stem cells in hydrogels with glucosamine. Biomaterials 2006;27:6015-23

[113] Huang, CY, Hagar, KL, Frost, LE, Sun, Y and Cheung, HS. Effects of cyclic compressive loading on chondrogenesis of rabbit bone-marrow derived mesenchymal stem cells. Stem Cells 2004;22:313-23

[114] Terraciano, V, Hwang, N, Moroni, L, Park, HB, Zhang, Z, Mizrahi, J, Seliktar, D and Elisseeff, J. Differential response of adult and embryonic mesenchymal progenitor cells to mechanical compression in hydrogels. Stem Cells 2007;25:2730-8

[115] Duplomb, L, Dagouassat, M, Jourdon, P and Heymann, D. Concise review: embryonic stem cells: a new tool to study osteoblast and osteoclast differentiation. Stem Cells 2007;25:544-52

[116] Goodman, ML, Chen, S, Yang, FC and Chan, RJ. Novel Method of Murine Embryonic Stem Cell-Derived Osteoclast Development. Stem Cells Dev 2008;

[117] Hemmi, H, Okuyama, H, Yamane, T, Nishikawa, S, Nakano, T, Yamazaki, H, Kunisada, T and Hayashi, S. Temporal and spatial localization of osteoclasts in colonies from embryonic stem cells. Biochem Biophys Res Commun 2001;280:526-34

[118] Okuyama, H, Tsuneto, M, Yamane, T, Yamazaki, H and Hayashi, S. Discrete types of osteoclast precursors can be generated from embryonic stem cells. Stem Cells 2003;21:670-80

[119] Suzuki, A and Nakano, T. Development of hematopoietic cells from embryonic stem cells. Int J Hematol 2001;73:1-5

[120] Tsuneto, M, Tominaga, A, Yamazaki, H, Yoshino, M, Orkin, SH and Hayashi, S. Enforced expression of PU.1 rescues osteoclastogenesis from embryonic stem cells lacking Tal-1. Stem Cells 2005;23:134-43

[121] Tsuneto, M, Yamazaki, H, Yoshino, M, Yamada, T and Hayashi, S. Ascorbic acid promotes osteoclastogenesis from embryonic stem cells. Biochem Biophys Res Commun 2005;335:1239-46

[122] Yamane, T, Kunisada, T and Hayashi, S. Embryonic stem cells as a model for studying osteoclast lineage development. Methods Mol Biol 2002;185:97-106

[123] Yamane, T, Kunisada, T, Yamazaki, H, Era, T, Nakano, T and Hayashi, SI. Development of osteoclasts from embryonic stem cells through a pathway that is c-fms but not c-kit dependent. Blood 1997;90:3516-23 
[124] Yamane, T, Kunisada, T, Yamazaki, H, Nakano, T, Orkin, SH and Hayashi, SI. Sequential requirements for SCL/tal-1, GATA-2, macrophage colony-stimulating factor, and osteoclast differentiation factor/osteoprotegerin ligand in osteoclast development. Exp Hematol 2000;28:833-40

[125] Nakashima, K, Zhou, X, Kunkel, G, Zhang, Z, Deng, JM, Behringer, RR and de Crombrugghe, B. The novel zinc finger-containing transcription factor osterix is required for osteoblast differentiation and bone formation. Cell 2002;108:17-29

[126] Ducy, P, Zhang, R, Geoffroy, V, Ridall, AL and Karsenty, G. Osf2/Cbfa1: a transcriptional activator of osteoblast differentiation. Cell 1997;89:747-54

[127] Buttery, LD, Bourne, S, Xynos, JD, Wood, H, Hughes, FJ, Hughes, SP, Episkopou, V and Polak, JM. Differentiation of osteoblasts and in vitro bone formation from murine embryonic stem cells. Tissue Eng 2001;7:89-99

[128] Jaiswal, N, Haynesworth, SE, Caplan, Al and Bruder, SP. Osteogenic differentiation of purified, cultureexpanded human mesenchymal stem cells in vitro. J Cell Biochem 1997;64:295-312

[129] Jukes, JM, Both, SK, Leusink, A, Sterk, LM, van Blitterswijk, CA and de Boer, J. Endochondral bone tissue engineering using embryonic stem cells. Proc Natl Acad Sci U S A 2008;105:6840-5

[130] Sottile, V, Thomson, A and McWhir, J. In vitro osteogenic differentiation of human ES cells. Cloning Stem Cells 2003;5:149-55

[131] Handschel, J, Berr, K, Depprich, RA, Kubler, NR, Naujoks, C, Wiesmann, HP, Ommerborn, MA and Meyer, $\mathrm{U}$. Induction of osteogenic markers in differentially treated cultures of embryonic stem cells. Head Face Med 2008;4:10

[132] Shimko, DA, Burks, CA, Dee, KC and Nauman, EA. Comparison of in vitro mineralization by murine embryonic and adult stem cells cultured in an osteogenic medium. Tissue Eng 2004;10:1386-98

[133] Cao, T, Heng, BC, Ye, CP, Liu, H, Toh, WS, Robson, P, Li, P, Hong, YH and Stanton, LW. Osteogenic differentiation within intact human embryoid bodies result in a marked increase in osteocalcin secretion after 12 days of in vitro culture, and formation of morphologically distinct nodule-like structures. Tissue Cell 2005;37:325-34

[134] Chaudhry, GR, Yao, D, Smith, A and Hussain, A. Osteogenic Cells Derived From Embryonic Stem Cells Produced Bone Nodules in Three-Dimensional Scaffolds. J Biomed Biotechnol 2004;2004:203-210

[135] Dani, C, Smith, AG, Dessolin, S, Leroy, P, Staccini, L, Villageois, P, Darimont, C and Ailhaud, G. Differentiation of embryonic stem cells into adipocytes in vitro. J Cell Sci 1997;110 ( Pt 11):1279-85

[136] Phillips, BW, Belmonte, N, Vernochet, C, Ailhaud, G and Dani, C. Compactin enhances osteogenesis in murine embryonic stem cells. Biochem Biophys Res Commun 2001;284:478-84

[137] Mundy, G, Garrett, R, Harris, S, Chan, J, Chen, D, Rossini, G, Boyce, B, Zhao, M and Gutierrez, G. Stimulation of bone formation in vitro and in rodents by statins. Science 1999;286:1946-9

[138] Bielby, RC, Christodoulou, IS, Pryce, RS, Radford, WJ, Hench, LL and Polak, JM. Time- and concentrationdependent effects of dissolution products of 585 sol-gel bioactive glass on proliferation and differentiation of murine and human osteoblasts. Tissue Eng 2004;10:1018-26

[139] Bielby, RC, Pryce, RS, Hench, LL and Polak, JM. Enhanced derivation of osteogenic cells from murine embryonic stem cells after treatment with ionic dissolution products of $58 \mathrm{~S}$ bioactive sol-gel glass. Tissue Eng 2005;11:479-88

[140] Bourne, S, Polak, JM, Hughes, SP and Buttery, LD. Osteogenic differentiation of mouse embryonic stem cells: differential gene expression analysis by cDNA microarray and purification of osteoblasts by cadherin-11 magnetically activated cell sorting. Tissue Eng 2004;10:796-806

[141] Bielby, RC, Boccaccini, AR, Polak, JM and Buttery, LD. In vitro differentiation and in vivo mineralization of osteogenic cells derived from human embryonic stem cells. Tissue Eng 2004;10:1518-25

[142] zur Nieden, NI, Kempka, G and Ahr, HJ. In vitro differentiation of embryonic stem cells into mineralized osteoblasts. Differentiation 2003;71:18-27

[143] zur Nieden, NI, Price, FD, Davis, LA, Everitt, RE and Rancourt, DE. Gene profiling on mixed embryonic stem cell populations reveals a biphasic role for beta-catenin in osteogenic differentiation. Mol Endocrinol 2007;21:674-85

[144] Ahn, SE, Kim, S, Park, KH, Moon, SH, Lee, HJ, Kim, GJ, Lee, YJ, Park, KH, Cha, KY and Chung, HM. Primary bone-derived cells induce osteogenic differentiation without exogenous factors in human embryonic stem cells. Biochem Biophys Res Commun 2006;340:403-8

[145] Heng, BC, Toh, WS, Pereira, BP, Tan, BL, Fu, X, Liu, H, Lu, K, Yeo, JF and Cao, T. An autologous cell lysate extract from human embryonic stem cell (hESC) derived osteoblasts can enhance osteogenesis of $h E S C$. Tissue Cell 2008;

[146] Hwang, YS, Randle, WL, Bielby, RC, Polak, JM and Mantalaris, A. Enhanced derivation of osteogenic cells from murine embryonic stem cells after treatment with HepG2-conditioned medium and modulation of 
the embryoid body formation period: application to skeletal tissue engineering. Tissue Eng 2006;12:138192

[147] Hwang, YS, Polak, JM and Mantalaris, A. In Vitro Direct Osteogenesis of Murine Embryonic Stem Cells Without Embryoid Body Formation. Stem Cells Dev 2008;

[148] Hwang, YS, Polak, JM and Mantalaris, A. In Vitro Direct Chondrogenesis of Murine Embryonic Stem Cells by Bypassing Embryoid Body Formation. Stem Cells Dev 2008;

[149] Karp, JM, Ferreira, LS, Khademhosseini, A, Kwon, AH, Yeh, J and Langer, RS. Cultivation of human embryonic stem cells without the embryoid body step enhances osteogenesis in vitro. Stem Cells 2006;24:835-43

[150] Duplomb, L, Dagouassat, M, Jourdon, P and Heymann, D. Differentiation of osteoblasts from mouse embryonic stem cells without generation of embryoid body. In Vitro Cell Dev Biol Anim 2007;43:21-4

[151] Karner, E, Unger, C, Sloan, AJ, Ahrlund-Richter, L, Sugars, RV and Wendel, M. Bone matrix formation in osteogenic cultures derived from human embryonic stem cells in vitro. Stem Cells Dev 2007;16:39-52

[152] Garreta, E, Genove, E, Borros, S and Semino, CE. Osteogenic differentiation of mouse embryonic stem cells and mouse embryonic fibroblasts in a three-dimensional self-assembling peptide scaffold. Tissue Eng 2006;12:2215-27

[153] Tremoleda, JL, Forsyth, NR, Khan, NS, Wojtacha, D, Christodoulou, I, Tye, BJ, Racey, SN, Collishaw, S, Sottile, V, Thomson, AJ, Simpson, AH, Noble, BS and McWhir, J. Bone tissue formation from human embryonic stem cells in vivo. Cloning Stem Cells 2008;10:119-32

[154] Kim, S, Kim, SS, Lee, SH, Eun Ahn, S, Gwak, SJ, Song, JH, Kim, BS and Chung, HM. In vivo bone formation from human embryonic stem cell-derived osteogenic cells in poly(d,l-lactic-co-glycolic acid)/hydroxyapatite composite scaffolds. Biomaterials 2008;29:1043-53

[155] Hegert, C, Kramer, J, Hargus, G, Muller, J, Guan, K, Wobus, AM, Muller, PK and Rohwedel, J. Differentiation plasticity of chondrocytes derived from mouse embryonic stem cells. J Cell Sci 2002;115:4617-28

[156] Kramer, J, Bohrnsen, F, Schlenke, P and Rohwedel, J. Stem cell-derived chondrocytes for regenerative medicine. Transplant Proc 2006;38:762-5

[157] Randle, WL, Cha, JM, Hwang, YS, Chan, KL, Kazarian, SG, Polak, JM and Mantalaris, A. Integrated 3dimensional expansion and osteogenic differentiation of murine embryonic stem cells. Tissue Eng 2007; 13:2957-70

[158] Tielens, S, Declercq, H, Gorski, T, Lippens, E, Schacht, E and Cornelissen, M. Gelatin-based microcarriers as embryonic stem cell delivery system in bone tissue engineering: an in-vitro study. Biomacromolecules 2007;8:825-32

[159] Taylor, CJ, Bolton, EM, Pocock, S, Sharples, LD, Pedersen, RA and Bradley, JA. Banking on human embryonic stem cells: estimating the number of donor cell lines needed for HLA matching. Lancet 2005;366:2019-25

[160] Takahashi, K, Tanabe, K, Ohnuki, M, Narita, M, Ichisaka, T, Tomoda, K and Yamanaka, S. Induction of pluripotent stem cells from adult human fibroblasts by defined factors. Cell 2007;131:861-72

[161] Yu, J, Vodyanik, MA, Smuga-Otto, K, Antosiewicz-Bourget, J, Frane, JL, Tian, S, Nie, J, Jonsdottir, GA, Ruotti, V, Stewart, R, Slukvin, II and Thomson, JA. Induced pluripotent stem cell lines derived from human somatic cells. Science 2007;318:1917-20

[162] Wakitani, S, Takaoka, K, Hattori, T, Miyazawa, N, Iwanaga, T, Takeda, S, Watanabe, TK and Tanigami, A. Embryonic stem cells injected into the mouse knee joint form teratomas and subsequently destroy the joint. Rheumatology (Oxford) 2003;42:162-5

[163] Wakitani, S, Aoki, H, Harada, Y, Sonobe, M, Morita, Y, Mu, Y, Tomita, N, Nakamura, Y, Takeda, S, Watanabe, TK and Tanigami, A. Embryonic stem cells form articular cartilage, not teratomas, in osteochondral defects of rat joints. Cell Transplant 2004;13:331-6

[164] Nakajima, M, Wakitani, S, Harada, Y, Tanigami, A and Tomita, N. In vivo mechanical condition plays an important role for appearance of cartilage tissue in ES cell transplanted joint. J Orthop Res 2008;26:10-7

[165] Hiemstra, C, Zhong, Z, Li, L, Dijkstra, PJ and Feijen, J. In-situ formation of biodegradable hydrogels by stereocomplexation of PEG-(PLLA)8 and PEG-(PDLA)8 star block copolymers. Biomacromolecules 2006; 7:2790-5

[166] Elbert, DL, Pratt, AB, Lutolf, MP, Halstenberg, S and Hubbell, JA. Protein delivery from materials formed by self-selective conjugate addition reactions. J Control Release 2001;76:11-25

[167] Metters, A and Hubbell, J. Network formation and degradation behavior of hydrogels formed by Michael-type addition reactions. Biomacromolecules 2005;6:290-301

[168] Lutolf, MP and Hubbell, JA. Synthesis and physicochemical characterization of end-linked poly(ethylene glycol)-co-peptide hydrogels formed by Michael-type addition. Biomacromolecules 2003;4:713-22 
[169] Lutolf, MP, Tirelli, N, Cerritelli, S, Cavalli, L and Hubbell, JA. Systematic modulation of Michael-type reactivity of thiols through the use of charged amino acids. Bioconjug Chem 2001;12:1051-6

[170] Hiemstra, C, Aa, LJ, Zhong, Z, Dijkstra, PJ and Feijen, J. Rapidly in situ-forming degradable hydrogels from dextran thiols through Michael addition. Biomacromolecules 2007;8:1548-56

[171] Hiemstra, C, van der Aa, LJ, Zhong, ZY, Dijkstra, PJ and Feijen, J. Novel in situ forming, degradable dextran hydrogels by Michael addition chemistry: Synthesis, rheology, and degradation. Macromolecules 2007;40:1165-1173

[172] Van Tomme, SR and Hennink, WE. Biodegradable dextran hydrogels for protein delivery applications. Expert Rev Med Devices 2007;4:147-64

[173] Jin, R, Hiemstra, C, Zhong, Z and Feijen, J. Enzyme-mediated fast in situ formation of hydrogels from dextran-tyramine conjugates. Biomaterials 2007;28:2791-800

[174] Van Tomme, SR, van Steenbergen, MJ, De Smedt, SC, van Nostrum, CF and Hennink, WE. Self-gelling hydrogels based on oppositely charged dextran microspheres. Biomaterials 2005;26:2129-35

[175] Hiemstra, C, Zhong, Z, van Steenbergen, MJ, Hennink, WE and Feijen, J. Release of model proteins and basic fibroblast growth factor from in situ forming degradable dextran hydrogels. J Control Release 2007;122:71-8

[176] Smith, AG and Hooper, ML. Buffalo rat liver cells produce a diffusible activity which inhibits the differentiation of murine embryonal carcinoma and embryonic stem cells. Dev Biol 1987;121:1-9

[177] Zuk, PA, Zhu, M, Mizuno, H, Huang, J, Futrell, JW, Katz, AJ, Benhaim, P, Lorenz, HP and Hedrick, MH. Multilineage cells from human adipose tissue: implications for cell-based therapies. Tissue Eng 2001;7:211-28

[178] Jukes, JM, Both, SK, van Blitterswijk, CA and de Boer, J. Potential of embryonic stem cells for in vivo bone regeneration. Regen Med 2008;3:783-5

[179] Mahmood, TA, de Jong, R, Riesle, J, Langer, R and van Blitterswijk, CA. Adhesion-mediated signal transduction in human articular chondrocytes: the influence of biomaterial chemistry and tenascin- $C$. Exp Cell Res 2004;301:179-88

[180] Malda, J, Woodfield, TB, van der Vloodt, F, Wilson, C, Martens, DE, Tramper, J, van Blitterswijk, CA and Riesle, J. The effect of PEGT/PBT scaffold architecture on the composition of tissue engineered cartilage. Biomaterials 2005;26:63-72

[181] Woodfield, TB, Malda, J, de Wijn, J, Peters, F, Riesle, J and van Blitterswijk, CA. Design of porous scaffolds for cartilage tissue engineering using a three-dimensional fiber-deposition technique. Biomaterials 2004;25:4149-61

[182] Moroni, L, de Wijn, JR and van Blitterswijk, CA. 3D fiber-deposited scaffolds for tissue engineering: influence of pores geometry and architecture on dynamic mechanical properties. Biomaterials 2006;27:974-85

[183] Sakkers, RJ, de Wijn, JR, Dalmeyer, RA, van Blitterswijk, CA and Brand, R. Evaluation of copolymers of polyethylene oxide and polybutylene terephthalate (polyactive): mechanical behaviour. J Mater Sci Mater Med 1998;9:375-9

[184] Claase, MB, Grijpma, DW, Mendes, SC, De Bruijn, JD and Feijen, J. Porous PEOT/PBT scaffolds for bone tissue engineering: preparation, characterization, and in vitro bone marrow cell culturing. J Biomed Mater Res A 2003;64:291-300

[185] Woodfield, TB, Miot, S, Martin, I, van Blitterswijk, CA and Riesle, J. The regulation of expanded human nasal chondrocyte re-differentiation capacity by substrate composition and gas plasma surface modification. Biomaterials 2006;27:1043-53

[186] Livak, KJ and Schmittgen, TD. Analysis of relative gene expression data using real-time quantitative PCR and the 2(-Delta Delta C(T)) Method. Methods 2001;25:402-8

[187] Simpson, DA, Feeney, S, Boyle, C and Stitt, AW. Retinal VEGF MRNA measured by SYBR green I fluorescence: A versatile approach to quantitative PCR. Mol Vis 2000;6:178-83

[188] Benya, PD and Shaffer, JD. Dedifferentiated chondrocytes reexpress the differentiated collagen phenotype when cultured in agarose gels. Cell 1982;30:215-24

[189] Mauck, RL, Soltz, MA, Wang, CC, Wong, DD, Chao, PH, Valhmu, WB, Hung, CT and Ateshian, GA. Functional tissue engineering of articular cartilage through dynamic loading of chondrocyte-seeded agarose gels. J Biomech Eng 2000;122:252-60

[190] Buschmann, MD, Gluzband, YA, Grodzinsky, AJ and Hunziker, EB. Mechanical compression modulates matrix biosynthesis in chondrocyte/agarose culture. J Cell Sci 1995;108 ( Pt 4):1497-508

[191] Radder, AM, Leenders, H and van Blitterswijk, CA. Application of porous PEO/PBT copolymers for bone replacement. J Biomed Mater Res 1996;30:341-51 
[192] Mendes, SC, Bezemer, J, Claase, MB, Grijpma, DW, Bellia, G, Degli-Innocenti, F, Reis, RL, de Groot, K, van Blitterswijk, CA and de Bruijn, JD. Evaluation of two biodegradable polymeric systems as substrates for bone tissue engineering. Tissue Eng 2003;9 Suppl 1:S91-101

[193] Beumer, GJ, van Blitterswijk, CA and Ponec, M. Biocompatibility of a biodegradable matrix used as a skin substitute: an in vivo evaluation. J Biomed Mater Res 1994;28:545-52

[194] Mensik, I, Lamme, EN, Riesle, J and Brychta, P. Effectiveness and Safety of the PEGT/PBT Copolymer Scaffold as Dermal Substitute in Scar Reconstruction Wounds (Feasibility Trial). Cell Tissue Bank 2002;3:245-53

[195] El-Ghalbzouri, A, Lamme, EN, van Blitterswijk, C, Koopman, J and Ponec, M. The use of PEGT/PBT as a dermal scaffold for skin tissue engineering. Biomaterials 2004;25:2987-96

[196] Wang, HJ, Bertrand-De Haas, M, Riesle, J, Lamme, E and Van Blitterswijk, CA. Tissue engineering of dermal substitutes based on porous PEGT/PBT copolymer scaffolds: comparison of culture conditions. J Mater Sci Mater Med 2003;14:235-40

[197] Mahmood, TA, Shastri, VP, van Blitterswijk, CA, Langer, R and Riesle, J. Tissue engineering of bovine articular cartilage within porous poly(ether ester) copolymer scaffolds with different structures. Tissue Eng 2005;11:1244-53

[198] Xiao, YL, Riesle, J and Van Blitterswijk, CA. Static and dynamic fibroblast seeding and cultivation in porous PEO/PBT scaffolds. J Mater Sci Mater Med 1999;10:773-7

[199] Wendt, D, Marsano, A, Jakob, M, Heberer, M and Martin, I. Oscillating perfusion of cell suspensions through three-dimensional scaffolds enhances cell seeding efficiency and uniformity. Biotechnol Bioeng 2003;84:205-14

[200] Kim, BS, Putnam, AJ, Kulik, TJ and Mooney, DJ. Optimizing seeding and culture methods to engineer smooth muscle tissue on biodegradable polymer matrices. Biotechnol Bioeng 1998;57:46-54

[201] Malda, J, Woodfield, TB, van der Vloodt, F, Kooy, FK, Martens, DE, Tramper, J, van Blitterswijk, CA and Riesle, J. The effect of PEGT/PBT scaffold architecture on oxygen gradients in tissue engineered cartilaginous constructs. Biomaterials 2004;25:5773-80

[202] Miot, S, Woodfield, T, Daniels, AU, Suetterlin, R, Peterschmitt, I, Heberer, M, van Blitterswijk, CA, Riesle, $\mathrm{J}$ and Martin, I. Effects of scaffold composition and architecture on human nasal chondrocyte redifferentiation and cartilaginous matrix deposition. Biomaterials 2005;26:2479-89

[203] Woodfield, TB, Van Blitterswijk, CA, De Wijn, J, Sims, TJ, Hollander, AP and Riesle, J. Polymer scaffolds fabricated with pore-size gradients as a model for studying the zonal organization within tissueengineered cartilage constructs. Tissue Eng 2005;11:1297-311

[204] Dang, SM, Gerecht-Nir, S, Chen, J, Itskovitz-Eldor, J and Zandstra, PW. Controlled, scalable embryonic stem cell differentiation culture. Stem Cells 2004;22:275-82

[205] Maguire, T, Novik, E, Schloss, R and Yarmush, M. Alginate-PLL microencapsulation: effect on the differentiation of embryonic stem cells into hepatocytes. Biotechnol Bioeng 2006;93:581-91

[206] Mouw, JK, Case, ND, Guldberg, RE, Plaas, AH and Levenston, ME. Variations in matrix composition and GAG fine structure among scaffolds for cartilage tissue engineering. Osteoarthritis Cartilage 2005;13:828-36

[207] Bradham, DM, Passaniti, A and Horton, WE, Jr. Mesenchymal cell chondrogenesis is stimulated by basement membrane matrix and inhibited by age-associated factors. Matrix Biol 1995;14:561-71

[208] Sohier, J, Haan, RE, de Groot, K and Bezemer, JM. A novel method to obtain protein release from porous polymer scaffolds: emulsion coating. J Control Release 2003;87:57-68

[209] Sohier, J, Vlugt, TJ, Cabrol, N, Van Blitterswijk, C, de Groot, K and Bezemer, JM. Dual release of proteins from porous polymeric scaffolds. J Control Release 2006;111:95-106

[210] De Bari, C, Dell'Accio, F and Luyten, FP. Failure of in vitro-differentiated mesenchymal stem cells from the synovial membrane to form ectopic stable cartilage in vivo. Arthritis Rheum 2004;50:142-50

[211] Lin, Y, Luo, E, Chen, X, Liu, L, Qiao, J, Yan, Z, Li, Z, Tang, W, Zheng, X and Tian, W. Molecular and cellular characterization during chondrogenic differentiation of adipose tissue-derived stromal cells in vitro and cartilage formation in vivo. J Cell Mol Med 2005;9:929-39

[212] Teramoto, K, Hara, Y, Kumashiro, Y, Chinzei, R, Tanaka, Y, Shimizu-Saito, K, Asahina, K, Teraoka, H and Arii, S. Teratoma formation and hepatocyte differentiation in mouse liver transplanted with mouse embryonic stem cell-derived embryoid bodies. Transplant Proc 2005;37:285-6

[213] Deacon, T, Dinsmore, J, Costantini, LC, Ratliff, J and Isacson, O. Blastula-stage stem cells can differentiate into dopaminergic and serotonergic neurons after transplantation. Exp Neurol 1998;149:28-41

[214] Baksh, D, Yao, R and Tuan, RS. Comparison of proliferative and multilineage differentiation potential of human mesenchymal stem cells derived from umbilical cord and bone marrow. Stem Cells 2007;25:138492 
[215] Sohier, J, Hamann, D, Koenders, M, Cucchiarini, M, Madry, H, van Blitterswijk, C, de Groot, K and Bezemer, JM. Tailored release of TGF-beta1 from porous scaffolds for cartilage tissue engineering. Int J Pharm 2007;332:80-9

[216] Williams, CG, Kim, TK, Taboas, A, Malik, A, Manson, P and Elisseeff, J. In vitro chondrogenesis of bone marrow-derived mesenchymal stem cells in a photopolymerizing hydrogel. Tissue Eng 2003;9:679-88

[217] Bosnakovski, D, Mizuno, M, Kim, G, Ishiguro, T, Okumura, M, Iwanaga, T, Kadosawa, T and Fujinaga, T. Chondrogenic differentiation of bovine bone marrow mesenchymal stem cells in pellet cultural system. Exp Hematol 2004;32:502-9

[218] Li, WJ, Tuli, R, Huang, X, Laquerriere, P and Tuan, RS. Multilineage differentiation of human mesenchymal stem cells in a three-dimensional nanofibrous scaffold. Biomaterials 2005;26:5158-66

[219] Pineda, S, Pollack, A, Stevenson, S, Goldberg, V and Caplan, A. A semiquantitative scale for histologic grading of articular cartilage repair. Acta Anat (Basel) 1992;143:335-40

[220] O'Driscoll, SW, Keeley, FW and Salter, RB. Durability of regenerated articular cartilage produced by free autogenous periosteal grafts in major full-thickness defects in joint surfaces under the influence of continuous passive motion. A follow-up report at one year. J Bone Joint Surg Am 1988;70:595-606

[221] Moojen, DJ, Saris, DB, Auw Yang, KG, Dhert, WJ and Verbout, AJ. The correlation and reproducibility of histological scoring systems in cartilage repair. Tissue Eng 2002;8:627-34

[222] Grogan, SP, Barbero, A, Winkelmann, V, Rieser, F, Fitzsimmons, JS, O'Driscoll, S, Martin, I and MainilVarlet, P. Visual histological grading system for the evaluation of in vitro-generated neocartilage. Tissue Eng 2006;12:2141-9

[223] de Waard, H, Sonneveld, E, de Wit, J, Lange, RE, Hoeijmakers, JH, Vrieling, H and van der Horst, GT. Celltype-specific consequences of nucleotide excision repair deficiencies: Embryonic stem cells versus fibroblasts. DNA Repair (Amst) 2008;

[224] Yuan, H, Van Den Doel, M, Li, S, Van Blitterswijk, CA, De Groot, K and De Bruijn, JD. A comparison of the osteoinductive potential of two calcium phosphate ceramics implanted intramuscularly in goats. J Mater Sci Mater Med 2002;13:1271-5

[225] Heins, N, Englund, MC, Sjoblom, C, Dahl, U, Tonning, A, Bergh, C, Lindahl, A, Hanson, C and Semb, H. Derivation, characterization, and differentiation of human embryonic stem cells. Stem Cells 2004;22:36776

[226] Both, SK, van der Muijsenberg, AJ, van Blitterswijk, CA, de Boer, J and de Bruijn, JD. A rapid and efficient method for expansion of human mesenchymal stem cells. Tissue Eng 2007;13:3-9

[227] Sohier, J. Growth factor releasing scaffolds for cartilage tissue engineering. 2006

[228] Malda, J, van Blitterswijk, CA, Grojec, M, Martens, DE, Tramper, J and Riesle, J. Expansion of bovine chondrocytes on microcarriers enhances redifferentiation. Tissue Eng 2003;9:939-48

[229] Siddappa, R, Licht, R, van Blitterswijk, C and de Boer, J. Donor variation and loss of multipotency during in vitro expansion of human mesenchymal stem cells for bone tissue engineering. J Orthop Res 2007;25:1029-41

[230] Meijer, GJ, de Bruijn, JD, Koole, R and van Blitterswijk, CA. Cell-based bone tissue engineering. PLoS Med 2007; 4:e9

[231] Sekiya, I, Colter, DC and Prockop, DJ. BMP-6 enhances chondrogenesis in a subpopulation of human marrow stromal cells. Biochem Biophys Res Commun 2001;284:411-8

[232] Hjelle, K, Solheim, E, Strand, T, Muri, R and Brittberg, M. Articular cartilage defects in 1,000 knee arthroscopies. Arthroscopy 2002;18:730-4

[233] Mallon, BS, Park, KY, Chen, KG, Hamilton, RS and McKay, RD. Toward xeno-free culture of human embryonic stem cells. Int J Biochem Cell Biol 2006;38:1063-75

[234] Itskovitz-Eldor, J, Schuldiner, M, Karsenti, D, Eden, A, Yanuka, O, Amit, M, Soreq, H and Benvenisty, N. Differentiation of human embryonic stem cells into embryoid bodies compromising the three embryonic germ layers. Mol Med 2000;6:88-95

[235] Moroni, L, de Wijn, JR and van Blitterswijk, CA. Three-dimensional fiber-deposited PEOT/PBT copolymer scaffolds for tissue engineering: influence of porosity, molecular network mesh size, and swelling in aqueous media on dynamic mechanical properties. J Biomed Mater Res A 2005;75:957-65

[236] Moroni, L, Poort, G, Van Keulen, F, de Wijn, JR and van Blitterswijk, CA. Dynamic mechanical properties of 3D fiber-deposited PEOT/PBT scaffolds: an experimental and numerical analysis. J Biomed Mater Res A 2006;78:605-14

[237] Anderson, DG, Levenberg, S and Langer, R. Nanoliter-scale synthesis of arrayed biomaterials and application to human embryonic stem cells. Nat Biotechnol 2004;22:863-6

[238] Pelttari, K, Winter, A, Steck, E, Goetzke, K, Hennig, T, Ochs, BG, Aigner, T and Richter, W. Premature induction of hypertrophy during in vitro chondrogenesis of human mesenchymal stem cells correlates 
with calcification and vascular invasion after ectopic transplantation in SCID mice. Arthritis Rheum 2006;54:3254-66

[239] Fijnvandraat, AC, van Ginneken, AC, de Boer, PA, Ruijter, JM, Christoffels, VM, Moorman, AF and Lekanne Deprez, RH. Cardiomyocytes derived from embryonic stem cells resemble cardiomyocytes of the embryonic heart tube. Cardiovasc Res 2003;58:399-409

[240] Kronenberg, HM. PTHrP and skeletal development. Ann N Y Acad Sci 2006;1068:1-13

[241] Kafienah, W, Mistry, S, Dickinson, SC, Sims, TJ, Learmonth, I and Hollander, AP. Three-dimensional cartilage tissue engineering using adult stem cells from osteoarthritis patients. Arthritis Rheum 2007;56:177-87

[242] Kim, YJ, Kim, HJ and Im, GI. PTHrP promotes chondrogenesis and suppresses hypertrophy from both bone marrow-derived and adipose tissue-derived MSCs. Biochem Biophys Res Commun 2008;373:104-8

[243] Brederlau, A, Correia, AS, Anisimov, SV, Elmi, M, Paul, G, Roybon, L, Morizane, A, Bergquist, F, Riebe, I, Nannmark, U, Carta, M, Hanse, E, Takahashi, J, Sasai, Y, Funa, K, Brundin, P, Eriksson, PS and Li, JY. Transplantation of human embryonic stem cell-derived cells to a rat model of Parkinson's disease: effect of in vitro differentiation on graft survival and teratoma formation. Stem Cells 2006;24:1433-40

[244] Both, S. Embryonic stem cells in bone tissue engineering, Chapter 7. 2008

[245] Swijnenburg, RJ, Tanaka, M, Vogel, H, Baker, J, Kofidis, T, Gunawan, F, Lebl, DR, Caffarelli, AD, de Bruin, $\mathrm{JL}$, Fedoseyeva, EV and Robbins, RC. Embryonic stem cell immunogenicity increases upon differentiation after transplantation into ischemic myocardium. Circulation 2005;112:1166-72

[246] Nussbaum, J, Minami, E, Laflamme, MA, Virag, JA, Ware, CB, Masino, A, Muskheli, V, Pabon, L, Reinecke, $\mathrm{H}$ and Murry, CE. Transplantation of undifferentiated murine embryonic stem cells in the heart: teratoma formation and immune response. FASEB J 2007;21:1345-57

[247] Both, S. Embryonic stem cells in bone tissue engineering, Chapter 6. 2008

[248] Yu, J, Vodyanik, MA, Smuga-Otto, K, Antosiewicz-Bourget, J, Frane, JL, Tian, S, Nie, J, Jonsdottir, GA, Ruotti, V, Stewart, R, Slukvin, II and Thomson, JA. Induced Pluripotent Stem Cell Lines Derived from Human Somatic Cells. Science 2007; 


\section{DANKWOORD}

Eindelijk dan de pagina's aan het eind van dit proefschrift die iedereen als eerste leest. Voor mij vormen ze de afsluiting van een flink aantal jaren onderzoek en ontdekking, lezen en schrijven, hé kijk nou momenten en mislukte experimenten, frustraties en ook veel plezier. Om kort samen te vatten: Er is vooruitgang geboekt, maar er was meer vooruitgang geboekt als alles had meegezeten. Ik denk dat meeste AIOs dit wel herkennen. Gelukkig herkennen de meeste mensen ook wel mijn gehuppel door de gangen, en als ik stellingen had toegevoegd aan dit proefschrift dan zou de laatste stelling geweest zijn: Je kunt niet chagrijnig huppelen. Helaas maken huppelende hersenen het schrijven van een proefschrift niet erg efficiënt.

Traditiegetrouw bedank ik als eerste mijn promotor Clemens van Blitterswijk en co-promotor Jan de Boer. Clemens, met verbazing en bewondering zie ik hoe jij in ieder idee en ieder resultaat een nieuwe spin-off ziet, terwijl ik vooral zou denken: Wat een onderneming. Jan, als laatste van je eerste AIOs is hier dan mijn proefschrift. Ook al zag ik soms op tegen de werkbesprekingen, na het bediscussiëren van de resultaten had ik altijd weer zin in de nieuwe experimenten. Hoe direct je commentaar ook was en hoe rigoureus de rode pen stukken tekst ook doorstreepte, er volgden altijd tips en suggesties ter verbetering, en achteraf gezien was je kritiek bijna altijd terecht.

Op mijn eerste werkdag in Bilthoven werd ik opgevangen door Sanne. Dat was het eerste uur van de vele, vele uren die we samen hebben doorgebracht. Samen op 1 kantoor, samen in de flowkast, samen de maan de schuld geven als de embryonale stamcellen weer eens niet deden wat wij wilden (of had je er toch stiekem in getuft?), samen op 1 kamer tijdens de congressen, en uiteindelijk zelfs samen in 1 huis. Ik zag je op een gegeven moment letterlijk vaker dan Bertrand, maar gelukkig zijn we elkaar nooit zat geweest. Onze samenwerking heeft geresulteerd in het mooiste hoofdstuk van dit proefschrift, ons PNAS artikel. Ik kan me de afgelopen jaren op het lab niet zonder jou voorstellen, en ik vind het fijn dat je mijn paranimf wilt zijn. Doreen, ook met jou op 1 kantoor, vaak samen op het lab, en samen in 1 huis. Ik kijk met een grote glimlach terug op de eerste (voor jou schokkend open) vrouwen praat in de tuin bij IsoTis, de vis en vrouwen avonden aan de Pathmossingel, jouw jam-kook kunsten, en je volledige enthousiasme voor nieuwe hobby's.

Er waren natuurlijk veel meer mensen met wie ik samen op het lab heb gewerkt. Pratend in de flow in het kweeklab, pipetterend op het BC lab, of zingend op het histologielab (excuses voor de valse noten), de tijd met jullie was een stuk gezelliger dan alleen. In alfabetische volgorde: Aart en Clayton 
(de originele "oude" AIOs, oeps, daar val ik nu zelf ook onder), Aliz (Enzjeet, oh, je bedoelt Enschede), Ana en Hemant (succes met de muizen ESC kweken), Anand (die krullenpruik stond je erg stoer), Anindita (toch zwanger worden tijdens je AlO schap!), Anouk (ik vertrouw je mijn kweken altijd toe), Bernke (kom je niet even hierheen om je kweken te verversen?), de mensen van CellCoTec (dan was ik niet de enige die aan kraakbeen werkte), Hugo ( $\mathrm{mmm}$, sexy roze hardloopbroek), Hugo André (dikke kus, als jij het je vriendin niet vertelt, zeg ik niets tegen mijn vriend), Florence (IsoTis, Utwente, Zwitserland, Progentix: via een omweg toch weer terug in Bilthoven), Frank en Gustavo (de Wageningers die ook echt met bioreactoren werken), Ineke (het is weer tijd voor nieuwe kinderfoto's), Jacqueline (leuk hè, snijden in een koeienknie), Jeanine (kraakbeen, kraakbeen, geen bot), Jens (lunch in de kantine is echt mogelijk), Jeroen R (doe mij nog maar wat droge sarcastische Drentse humor in de carpool-panda), Jeroen L (als je je principes overboord gooit doe je het inderdaad goed), Jérôme (voor jou altijd Zjozjanèk), Jiaping en Yuan (Mr. Titanium en Mr. BCP), Joost de Bruijn (wonen op 2 plaatsen kan inderdaad extremer dan Harderwijk en Enschede), Joost de Wijn (biologen zijn geen chemici), Jos (Brisbane, leuke stad hè), Joyce (de eerste BMT student die het aandurfde te blijven), Jun (zullen we zo lekker gaan feesten?), Liang (succes met de laatste loodjes), Liliana (nog een huisgenoot-collega, die keurig laminaat kan leggen), Linda (heb je nog leuke muziekjes en wil je mee-eten?), Lorenzo (je bent vergeven, ik weet dat je 'jij hier!' bedoelde), Marcel (jippie, meer kraakbeen mensen), Nicolas (heel even in Bilthoven en direct door naar Enschede), Pamela (wanneer gaan we weer samen lunchen?), het Progentix-kippenhok (uit jullie kantoor kwam echt meer gekakel dan uit het onze), Ram (slapen op de achterbank van de panda), Remi (weer niet gelukt, opstaan met een prachtige zangstem), Riemke (niet alle meiden met kort haar lijken op elkaar), Roka (zit er nog een snoepje in de snoeppot?), Sandra (toppunt van Portugese gastvrijheid), Soledad (wow, een tweeling), Tara (kom er gezellig bij, in onze vrouwenkamer), Tim (45 sheets in 15 minuten past echt niet), Viola en Audrey (meer dan alleen de beste secretaresses). Alle nieuwe AIOs in de TR groep wens ik veel succes en plezier tijdens jullie onderzoek. Mijn 2 bachelor studenten Roderick en Theun, hopelijk hebben jullie wat opgestoken van jullie weken op het lab, en gaan jullie verder in de mooie wereld van TR.

Ik wil ook de collega's van PBM bedanken die de gelen uit hoofdstuk 3 hebben ontwikkeld: Mark, Christine en Hans, en hun begeleiders Jan Feijen, Piet Dijkstra en Zhiyuan Zhong.

Na Doreen en Sanne, en later Liliana, heb ik nu 2 niet-TR-huisgenootjes aan de Pathmossingel: Federica en Aimee (en Paul als ' 4 ' huisgenoot en bijna familie'). Ik zou maar een half jaar in het huis wonen, en het is nu al ruim 3 jaar, maar met jullie aanwezigheid is het niet zo erg om veel niet thuis 
te zijn. Ik hoop niet meer al te lang in het huis te wonen, maar geniet in die tijd wel van jullie Italiaanse en Canadese gezelligheid. Ciao dudes!

Onverwacht kwam er iets leuks kijken bij de verhuizing naar Enschede: een schaatsbaan, eerst om de hoek, en nu een prachtige 400 meter baan. Heerlijk even de aandacht niet op het proefschrift, maar op het ijs. Ik wil alle trainers en mede-schaatsers en skeeleraars van de Skeuvel bedanken voor de heerlijke in- en ontspanning op de baan en de leuke speel-oefeningen.

Natuurlijk was er ook veel gezelligheid buiten Enschede. Alle T34-ers die ondertussen over het hele land zijn uitgezworven. Al die rare bioprocessers, ook al zien we elkaar minder vaak dan in de tijden van de Woeste Hoeve en Annies, toch is het altijd weer gezellig als in die goede oude tijd als we elkaar zien.

De maandelijkse etentjes met de club van Valkenswaard waren niet alleen erg lekker, maar er was ook altijd tijd om de laatste roddels uit te wisselen. Janneke en Menno, heel veel plezier met jullie grootste resultaat tot nu toe. Erik, fijn dat ik altijd even bij je mocht zeuren op de msn. Fränzel, misschien wel de minst kakelende in ons kippenhok, maar ik ben vereerd dat je mijn paranimf wilt zijn. Zullen we voor ons 20 -jarig jubileum een weekje weg gaan?

Ik wil uiteraard ook mijn familie bedanken. Papa, het is niet erg als je niet zo veel snapt van dit proefschrift. Mijn onderzoek zou een stuk makkelijker zijn geweest als ik wist wat er in de skelettine van Madame Plijster zit. Zo snel een bot laten teruggroeien (ook al is het een vies drankje), daar zijn wij als tissue engineers jaloers op. Mijn zussen Marieke en Karlijne, gelukkig kan ik altijd bij jullie terecht voor advies, zussen-dingen en de telefoontjes vanuit de trein. Willem, Sara en Loes maken het huis in Valkenswaard heerlijk vol met de feestdagen. Ook wil ik paps en mams Schuiling, Eric, Meggie, Lucas, en Leon en Viveka bedanken voor de steun en peptalks tijdens de familie-etentjes. Alle ooms en tantes Jukes en van der Straten, dank voor jullie komst naar het verre Enschede.

Als laatste natuurlijk de grootste dank voor de grootste persoon in deze lange lijst. Lieve Bertrand, mijn stress en AlO-frustraties verdwenen altijd direct als ik even bij je op schoot mocht zitten. Gelukkig kon jij ook "hobbyen" achter jouw computer in de vele uren die ik achter mijn laptop zat. Zullen we nu fijn een stukje gaan huppelen met zijn tweetjes? Een hele dikke kus en een knuffel voor jou.

Jojanneke 


\section{CURRICULUM VITAE}

Jojanneke Jukes was born March $28^{\text {th }} 1978$ in Eindhoven. She grew up in Valkenswaard, where she attended high school (Hertog Jan College, later renamed Scholengemeenschap Were Di) and graduated in 1996. She went to Wageningen University to study bioprocess technology. She performed her first master thesis research at the Department of Cell biology and Immunology (Prof. Willem van Muiswinkel). Under the supervision of Dr. René Stet she worked on the Characterization of natural cytotoxicity receptors in carp and channel catfish. For her second master thesis, she investigated the Expression of White Spot Syndrome Virus proteins in bacteria. This research was performed at Intervet International BV in Boxmeer under the supervision of Dr. Mariëlle van Hulten from the Laboratory of Virology (Prof. Just Vlak), Wageningen University and Dr. Piet Nuijten from the Bacteriological R\&D group of Intervet. For her internship she went to the University of Queensland, Brisbane, Australia. At the Animal Cell Culture Group of the Department of Chemical Engineering, she performed a Rescue experiment to restore the MP phenotype to a pure HaSNPV FP isolate, supervised by Dr. Linda Lua.

In 2003, she graduated, and started her PhD research at the Institute for Biomedical Technology at the University of Twente, under the supervision of Dr. Jan de Boer and Prof. Dr. Clemens van Blitterswijk. The results of this research are described in this thesis. During the first years, the group's lab was located in Bilthoven, at the company Isotis BV. In 2006, the Tissue Regeneration group relocated to Enschede.

Since April 2008, she has been working as project manager for the "Translational excellence in Regenerative Medicine" (TeRM Smart Mix) program, at the Institute for Biomedical Technology at the University of Twente. As from September 2009, she will continue to work 3 days per week for the TeRM Smart Mix program, and work 2 days per week for MIRA, the Institute for Biomedical Technology and Technical Medicine, at the University of Twente. 